\title{
International Union of Basic and Clinical Pharmacology. LXXXIII: Classification of Prostanoid Receptors, Updating 15 Years of Progress
}

\author{
D. F. Woodward, R. L. Jones, and S. Narumiya
}

Department of Biological Sciences, Allergan Inc., Irvine, California (D.F.W.); Strathclyde Institute of Pharmacy \& Biomedical Sciences, University of Strathclyde, Glasgow, Scotland, United Kingdom (R.L.J.); and Department of Pharmacology, Kyoto University Faculty of Medicine, Yoshida, Kyoto, Japan (S.N.)

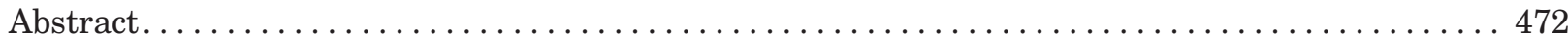

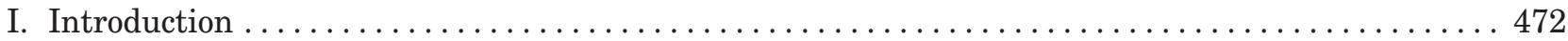

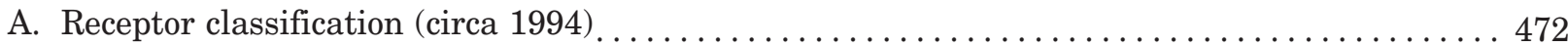

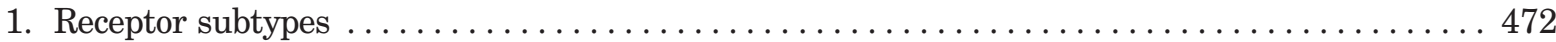

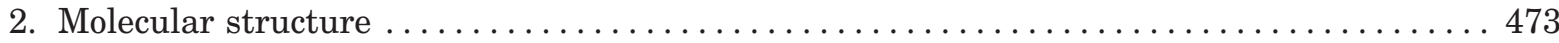

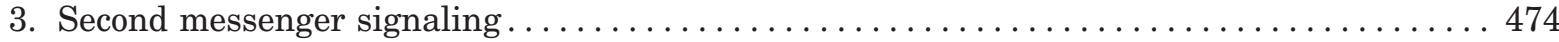

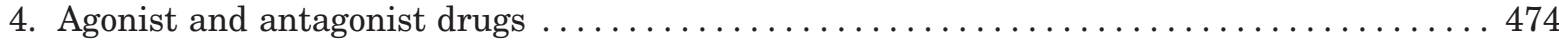

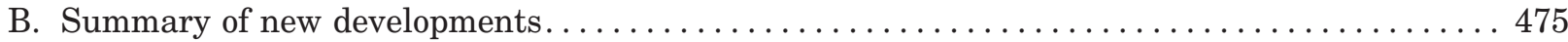

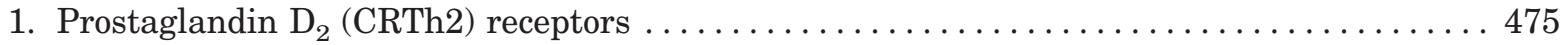

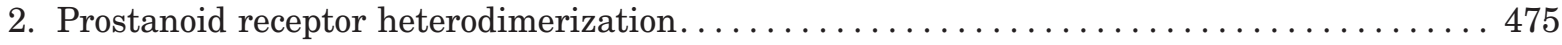

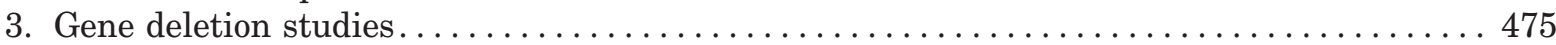

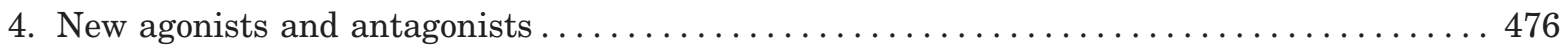

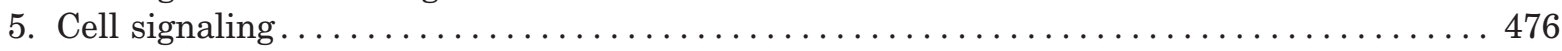

II. Receptor types, subtypes, and mRNA splicing variants $\ldots \ldots \ldots \ldots \ldots \ldots \ldots \ldots \ldots \ldots \ldots \ldots$

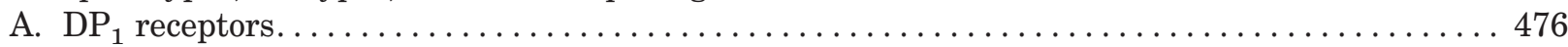

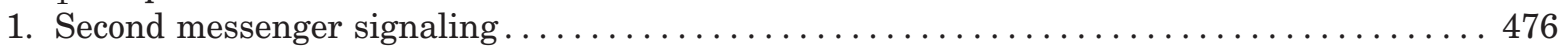

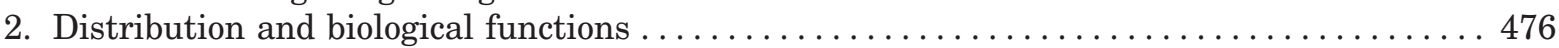

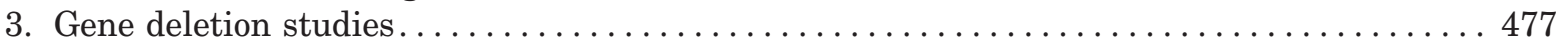

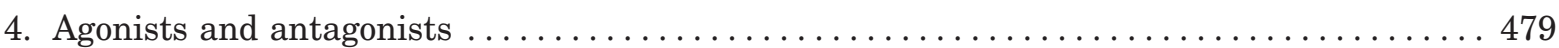

5. Therapeutics................................................ 480

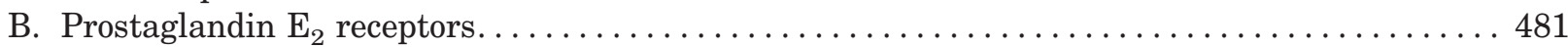

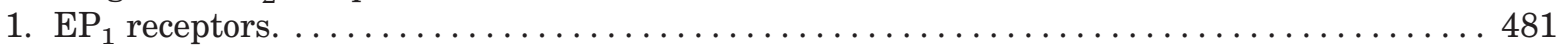

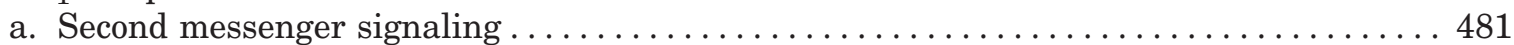

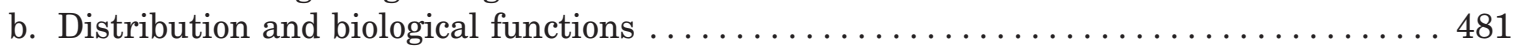

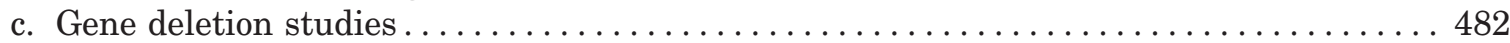

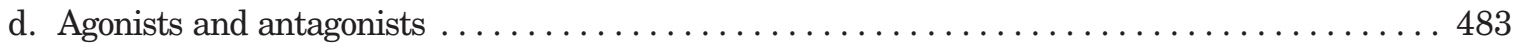

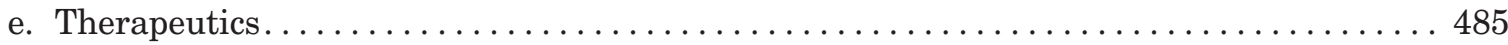

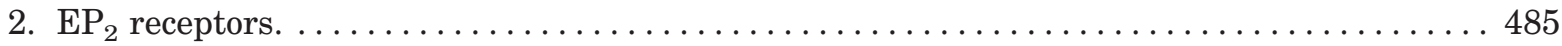

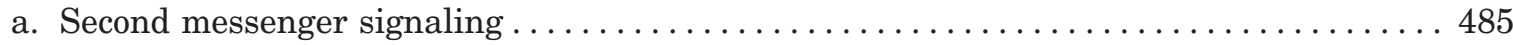

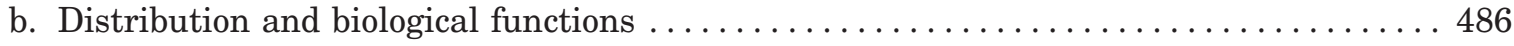

c. Gene deletion studies ....................................... 489

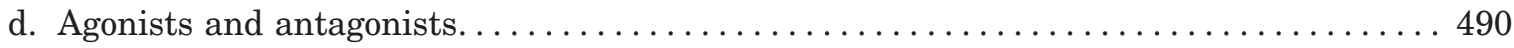

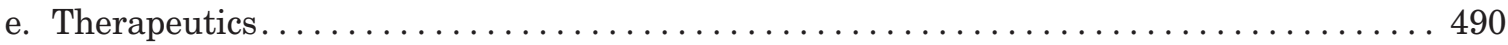

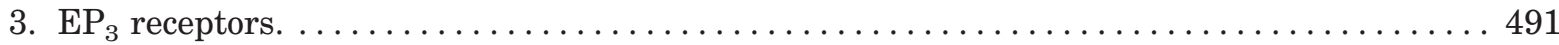

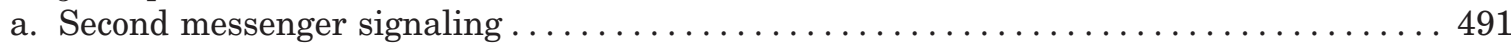

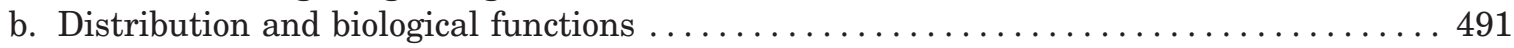

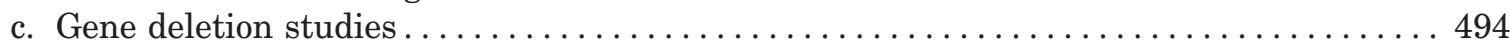

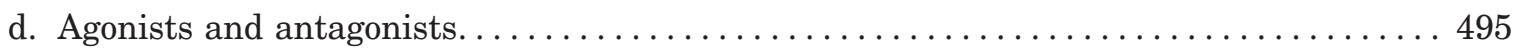

Address correspondence to: Dr. David F. Woodward, Dept. of Biological Sciences RD3-2B, Allergan, Inc., 2525 Dupont Dr., Irvine, CA 92612. E-mail: woodward_david@allergan.com

This article is available online at http://pharmrev.aspetjournals.org.

doi:10.1124/pr.110.003517. 


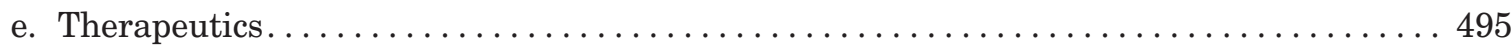

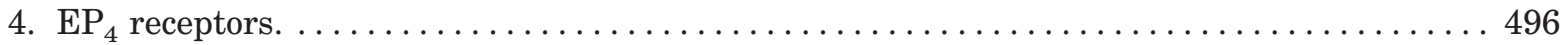

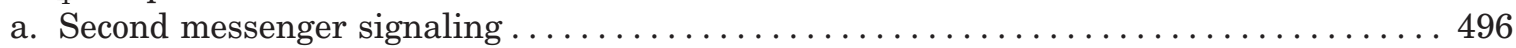

b. Distribution and biological functions $\ldots \ldots \ldots \ldots \ldots \ldots \ldots \ldots \ldots \ldots \ldots \ldots \ldots$

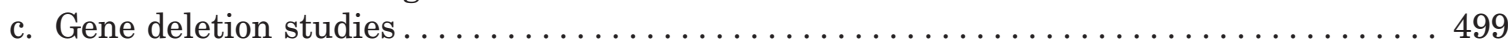

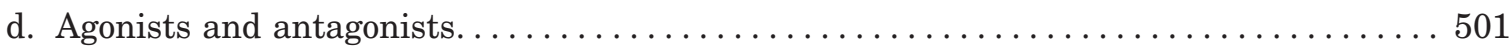

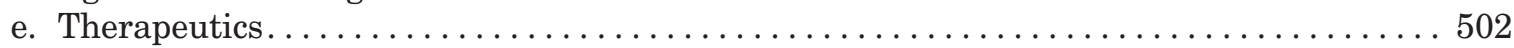

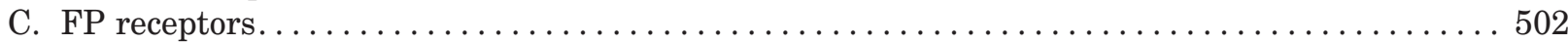

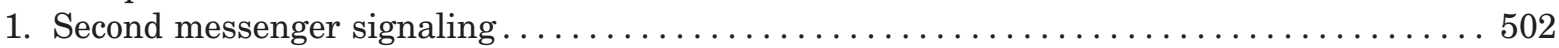

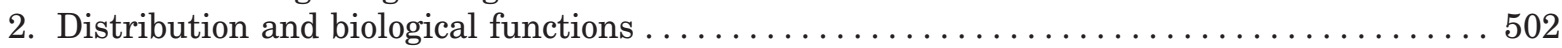

3. Gene deletion studies. ....................................... 504

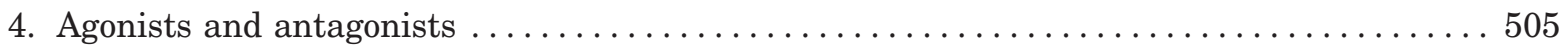

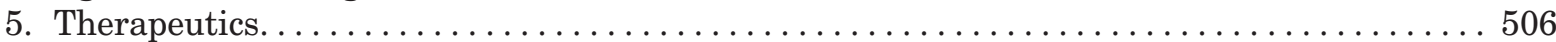

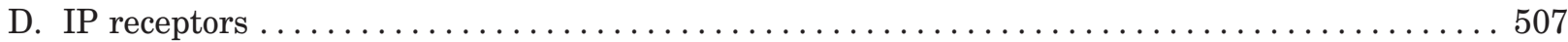

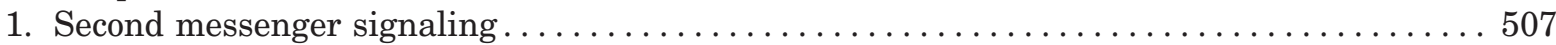

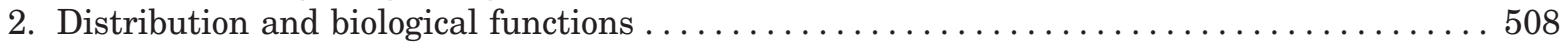

3. Gene deletion studies......................................... 509

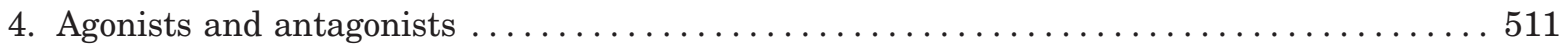

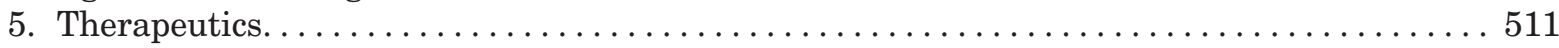

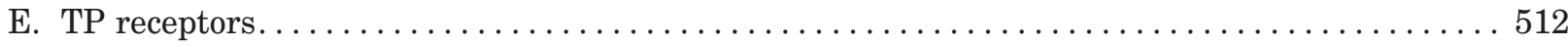

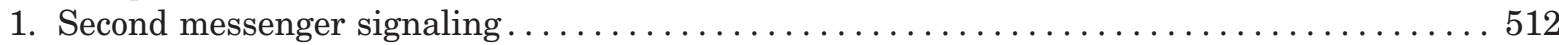

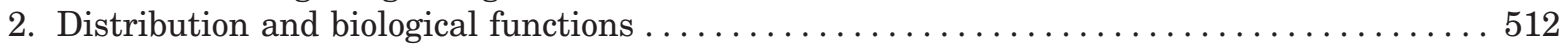

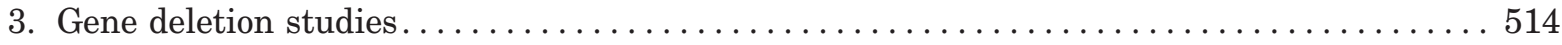

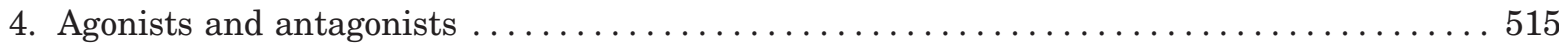

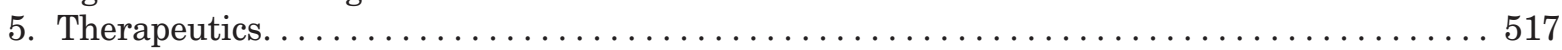

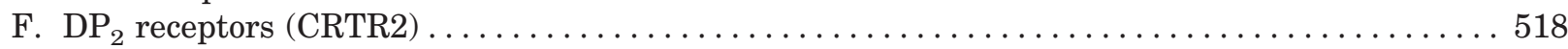

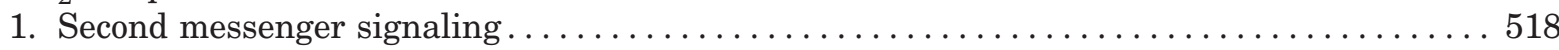

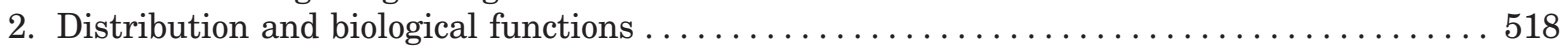

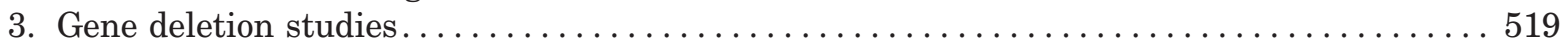

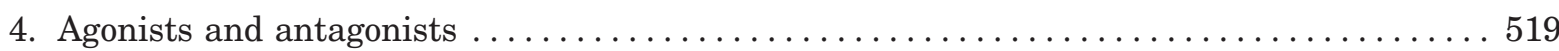

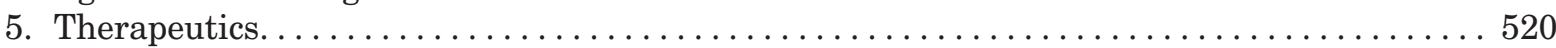

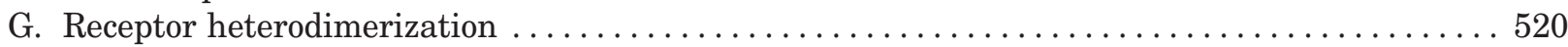

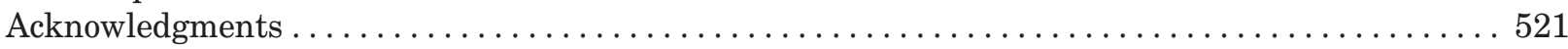

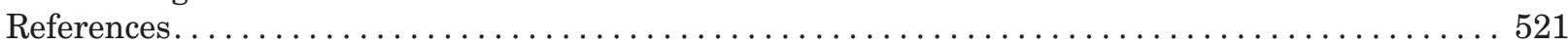

\begin{abstract}
It is now more than 15 years since the molecular structures of the major prostanoid receptors were elucidated. Since then, substantial progress has been achieved with respect to distribution and function, signal transduction mechanisms, and the design of agonists and antagonists (http://www.iuphar-db.org/ DATABASE/FamilyIntroductionForward? familyId=58). This review systematically details these advances. More recent developments in prostanoid receptor research are included. The $\mathrm{DP}_{2}$ receptor, also termed CRTH2, has little structural resemblance to $\mathrm{DP}_{1}$ and other receptors described in the original prostanoid receptor classifica-
\end{abstract}

\section{Introduction}

\section{A. Receptor Classification (circa 1994)}

1. Receptor Subtypes. The major prostaglandins $\left(\mathrm{PGs}^{1}\right)$, $\mathrm{PGD}_{2}, \mathrm{PGE}_{2}, \mathrm{PGF}_{2 \alpha}$, prostacyclin $\left(\mathrm{PGI}_{2}\right)$, and thromboxane $\mathrm{A}_{2}\left(\mathrm{TxA}_{2}\right)$ preferentially interact with dedicated receptors designated DP, EP, FP, IP, and TP, respectively (Kennedy et al., 1982; Coleman et al., 1984). Although tion. $\mathrm{DP}_{2}$ receptors are more closely related to chemoattractant receptors. Prostanoid receptors have also been found to heterodimerize with other prostanoid receptor subtypes and nonprostanoids. This may extend signal transduction pathways and create new ligand recognition sites: prostacyclin/thromboxane $A_{2}$ heterodimeric receptors for 8-epi-prostaglandin $\mathrm{E}_{2}$, wild-type/alternative (alt4) heterodimers for the prostaglandin FP receptor for bimatoprost and the prostamides. It is anticipated that the 15 years of research progress described herein will lead to novel therapeutic entities. largely based on functional studies using a limited range of agonists, and an even more limited range of antagonists, the original classification of prostanoid receptors has entirely withstood the tests of time and scrutiny. Prostanoid receptor subtypes were also proposed. Four subtypes of $\mathrm{EP}$ receptor $\left(\mathrm{EP}_{1}, \mathrm{EP}_{2}, \mathrm{EP}_{3}\right.$, and $\left.\mathrm{EP}_{4}\right)$ have been described (Coleman et al., 1994b). Two $\mathrm{PGD}_{2}$-sensitive receptors were suggested, but only $\mathrm{DP}_{1}$ was de- 
scribed in 1994, although diverse pharmacological evidence described a second $\mathrm{PGD}_{2}$-sensitve receptor (Jones, 1976a,b, 1978; Narumiya and Toda, 1985; Woodward et al., 1990, 1993b; Rangachari and Betti, 1993; Fernandes and Crankshaw, 1995).

2. Molecular Structure. Prostanoid receptors are G protein-coupled receptors. Although the overall homol-

${ }^{1}$ Abbreviations: AH 13205, trans-2-(4-(1-hydroxyhexyl)phenyl)-5oxocyclopentaneheptanoic acid; AH 23848, 7-(5-((1,1-biphenyl)-4yl)methoxy)-2-(4-morpholinyl)-3-oxocyclopentyl)-4-heptanoic acid; Akt, protein kinase B; AL-6556, 9-chloro-15-cyclohexyl-11,15-dihydroxy-3-oxa16,17,18,19,20-pentanor-5-prostenoic acid; ApoE, apolipoprotein E; BAL, bronchoalveolar lavage; Bay U 3405, ramatroban; BM-13177, sulotroban; BM-13505, daltroban; BMS-180291, ifetroban; BNP, brain natriuretic protein; BW 245C, 5-(6-carboxyhexyl)-1-(3-cyclohexyl-3-hydroxypropyl)hydantoin; BW A868C, 3-(3-[1,1'-biphenyl]-4-yl-3-hydroxypropyl)-2,5-dioxo-4imidazolidineheptanoic acid, ethyl ester; CAY-10399, "2-series" analog of butaprost-FA; CGRP, calcitonin gene-related peptide; CJ-023,423, $N$-(((2(4-(2-ethyl-4,6-dimethyl- $1 H$-imidazo(4,5-c)pyridin-1-yl)phenyl)ethyl)amino)carbonyl)-4-methylbenzenesulfonamide; CJ-42794, (S)-4-(1-(5-chloro-2(4-fluorophenyoxy)benzamido)ethyl)benzoic acid; CNS, central nervous system; COX, cyclooxygenase; CRTH2, chemoattractant receptorhomologous molecule expressed on Th2 cells; Cyr 61, cysteine-rich angiogenic protein 61; DC, dendritic cell; DNFB, dinitrofluorobenzene; EGF, epidermal growth factor; Epacs, exchange proteins activated by cAMP; ERK, extracellular signal regulated protein kinase; GR 63799X, (4benzamidophenyl)-( $Z$ )-7-[( $1 R, 2 R, 3 R)$-3-hydroxy-2-[(2R)-2-hydroxy-3phenoxypropoxy]-5-oxocyclopentyl] hept-5-enoate; GR-32191, vapiprost; GRK, G protein-coupled receptor kinase; HIF, hypoxia-inducible factor; HPGDS, hematopoietic prostaglandin D synthase; I-BOP, [1S[1 $\left.\left.\alpha, 2 \alpha(Z), 3 \beta\left(1 E, 3 S^{*}\right), 4 \alpha\right]\right]-7-[3-[3-h y d r o x y-4$-(4-iodophenoxy)-1-butenyl]-7oxabi-cyclo[2.2.1]hept-2-yl]5-heptenoic acid; ICI-192605, 6-(2-(2-chlorophenyl-4-hydroxyphenyl)-1,3-dioxan-5-yl)hexenoic acid; ICI-80205, 16-p-chlorophenoxy- $\omega$-tetranor PGE $\mathrm{P}_{2}$; ICI-81008, fluprostenol; IFN, interferon; IL, interleukin; IOP, intraocular pressure; iP, isoprostane; KO, knockout; KP-496, (2-( $N$-(4-(4-chlorobenzenesulfonylamino)butyl)- $N$-(3-(4isopropylthiazol-2-yl)methoxy)benzyl)sulfamoyl)benzoic acid; L-888,607, (9-((4-chlorophenyl)thio)-6-fluoro-2,3-dihydro- $1 H$-pyrrolo(1,2-a)indol-1yl)acetic acid; LC, Langerhans cell; LPS, lipopolysaccharide; MAP, mitogen-activated protein; MB-28767, 11-deoxy-16-phenoxy PGE ${ }_{1}$; MEK, mitogen-activated protein kinase kinase; MF-266-1, 1-(5-\{3-[2-(benzyloxy)5-chlorophenyl]-2-thienyl\}pyridin-3-yl)-2,2,2-trifluoroethane-1,1-diol; MK-0524, laropiprant; MLR, mixed lymphocyte reaction; NMDA, $N$-methyl-D-aspartate; ONO-11120, 11 $\alpha$-carba-12-(2'S-hydroxy-3'-phenylpropylamino)- $9 \alpha, 11 \alpha$-isopropylideno- $\omega$-octanor-prost-5Z-enoic acid; ONO-8711, 6-((2S,3S)-3-(4-chloro-2-methylphenysulfonylaminomethyl)bicyclo(2.2.2)octan-2-yl)-5Z-hexenoic acid; OVA, ovalbumin; PF-04475270, 5-(3-(2-(3-hydroxy-4-(3-(trifluoromethyl)phenyl)butyl)-5-oxopyrrolidin-1yl)propyl)thiophene-2-carboxylate; PG, prostaglandin; PGDS, prostaglandin D synthase; $\mathrm{PGI}_{2}$, prostacyclin; PI, phosphatidylinositol; PI3K, phosphoinositide-3-kinase; PKA, protein kinase A; PKC, protein kinase C; PLC, phospholipase C; PPAR, peroxisome proliferator-activated receptor; PTH, parathyroid hormone; RANKL, receptor activator of nuclear factor- $\kappa \mathrm{B}$ ligand; rc, recombinant; Rho, rhodopsin; S-145, 5,7-(3-phenylsulfonylamino(2.2.1)bicyclohept-2-yl)heptenoic acid; S-18886, terutroban; SC19220 , 8-chloro-dibenzo $(Z)[b, f][1,4]$ oxazepine-10 $(11 H)$-carboxylic acid, 2-acetylhydrazide; SQ-29548, 7-(3-((2-((phenylamino)carbonyl)hydrazino)methyl)-7-oxabicyclo(2.2.1)hept-2-yl)-5-heptenoic acid; STAT, signal transducer and activator of transcription; TGF, transforming growth factor; Th, T helper; THG-113.31, Ile-Leu-Gly-His-(D-Cit)-Asp-Tyr-Lys; TM, transmembrane domain; TNF, tumor necrosis factor; TR4979, butaprost; TS-022, (4-((1R,2S,3R,5R)-5-chloro-2-( $(S)-3$-cyclohexyl-3-hydroxyprop-1-ynyl)-3-hydroxycyclopentyl)butylthio) acetic acid monohydrate; $\mathrm{TxA}_{2}$, thromboxane $\mathrm{A}_{2}$; U-46619, 9-11-dideoxy-11 $\alpha, 9 \mathrm{a}$-epoxymethanoprostaglandin $\mathrm{F}_{2 \mathrm{a}}$; VEGF, vascular endothelial growth factor; WT, wild type. ogy between those receptors cloned in the 1990s was not high, there were several conserved regions. The first prostanoid receptor to be structurally identified was TP. This was achieved by purifying the TP receptor protein using the high-affinity, radiolabeled ligand S-145 (Ushikubi et al., 1989). Based on a partial amino acid sequence, the cDNA for the TP receptor was obtained (Hirata et al., 1991). The other prostanoid receptors were cloned by homology based screening and by 1994, $\mathrm{DP}_{1}, \mathrm{FP}, \mathrm{IP}$, and all $\mathrm{PGE}_{2}$-sensitive receptors had been structurally identified (Coleman et al., 1994b; Hirata et al., 1994; Nakagawa et al., 1994; Regan et al., 1994b).

The deduced amino acid sequences for human $\mathrm{DP}_{1}$, $\mathrm{EP}_{1-4}, \mathrm{FP}, \mathrm{IP}$, and TP receptors, together with $\mathrm{DP}_{2}$, are compared in Fig. 1. Hydrophobicity analysis of the sequences indicated seven membrane-spanning segments, an extracellular $\mathrm{N}$ terminus, and an intracellular - $\mathrm{COOH}$ terminus typical of rhodopsin-type, G-proteincoupled receptors. Regions of significant homology occur in the seventh transmembrane domain and the second extracellular loop. The highly conserved arginine in the seventh transmembrane (TM) domain has been proposed as the interaction site for the carboxylate group, which is common to all natural prostanoids. Additional determinants of prostanoid receptor binding have been suggested as follows. Two conserved Cys residues in the first and second extracellular loops are believed to form a disulfide bridge critical for stabilization of the receptor conformation (Narumiya et al., 1999). The second extracellular loop connecting TM4 and TM5 contains an invariant Trp-Cys-Phe triplet (Pierce et al., 1995). There are one or more consensus sequences for N-glycosylation of arginine residues in the amino terminal region $(\mathrm{Na}$ rumiya et al., 1999). N-glycosylation may also be important for ligand binding, at least for TP receptors (Chiang and Tai, 1998).

Studies on the molecular evolution of prostanoid receptors suggest a $\mathrm{PGE}_{2}$-sensitive entity as the ancestral receptor (Regan et al., 1994b; Boie et al., 1995; Toh et al., 1995; Foord et al., 1996). This may have occurred not only by gene duplication but also by chromosomal duplication (Duncan et al., 1995). These phylogenetic analyses suggested two major branches of prostanoid receptor evolution: one group $\mathrm{G}_{\mathrm{s}}$-coupled ( $\mathrm{DP}_{1}, \mathrm{EP}_{2}, \mathrm{EP}_{4}$, and IP) and the other group $\mathrm{G}_{\mathrm{i}^{-}}\left(\mathrm{EP}_{3}\right)$ or $\mathrm{G}_{\mathrm{q}}$-coupled $\left(\mathrm{EP}_{1}, \mathrm{FP}\right.$, and TP), providing three clusters (Table 1). For most receptors, mRNA splicing variants have been identified.

The $\mathrm{DP}_{2}$ receptor, although recognizing $\mathrm{PGD}_{2}$ as a primary natural ligand, has no significant homology with $\mathrm{DP}_{1}$ or other prostanoid receptors described in the original classification (Abe et al., 1999; Hirai et al., 2001; Hata et al., 2005). DP ${ }_{2}$ (CRTh2) is more closely related to chemoattractant receptors, such as $\mathrm{C}_{5 \alpha}$ and $N$-formyl peptide receptors (Abe et al., 1999; Hirai et al., 2001). The arginine residue in the seventh membranespanning domain, which is conserved in all receptors of the original classification, is not present in $\mathrm{DP}_{2}$ (Fig. 1). 
VLAPALDNSLCQA $\overline{F A F F M S F F G L S S T L Q L L A M A L E C W L S L G H P F F Y R R H I T L R L G A L V A P V V S A F S L A F C A L P F M G F G K F V Q Y C P G T W C F I Q M V H E E G S L S------V L G Y S V L Y S S L M A L L V L A T V ~}$ hCRTH2 GTT-----FCKLHSSIFFLNMFASGFLLSAISLDRCLQVVRPVWAQNHRTVAAAHKVCLVLWALAVLNTVPYFVFRDTISRLDGRIM hEP1 --APAGG--ACHFLGGCMVFFGLCPLLLGCGMAVERCVGVTRPLLHAARVSVARARLALAAVAAVALAVALLPLARVGRYELQYPGTW $\begin{array}{ll}\text { hEP2 } & \text { ALAPESR--ACTYFAFAMTFFSLATMLMLFAMALERYLSIGHPYFYQRRVSRSGGLAVLPVIYAVSLLFCSLPLLDYGQYVQYCPGTWCF } \\ \text { hEP3 } & \text { HIDPSGR--LCTFFGLTMTVFGLSSLFIASAMAVERALAIRAPHWYASHMKTRATRAVLLGVWLAVLAFALLPVLGVGOYVOWPGTWCF }\end{array}$ GLARGGP-ACIFGICMVFSGLCPLLGSVMAIERCIGVTKPIFHSTKITSKHVKMMLSGVCLFAVFIALLPILGHRDYKIQASRTWCFYNTEDIKDWE-------DRFYLLLFSFLGLLALGVSL GLARGGP-ALCDAFAFAMTFFGLASMLILFAMAVERCLALSHPYLYAQLDGPRCARLALPAIYAFCVLFCALPLLGLGQHQQYCPGSWCFLRMRWAQPG---------GAAFSLAYAGLVALLVAAIF
AVDPGCR--LCRFMGVVMIFFGLSPLLLGAAMASERYLGITRPFSRPAVASQRRAWATVGLVWAAALALGLLPLLGVGRYTVQYPGSWCFLTLG--AESG-------DVAFGLLFSMLGGLSVGLSF

VI

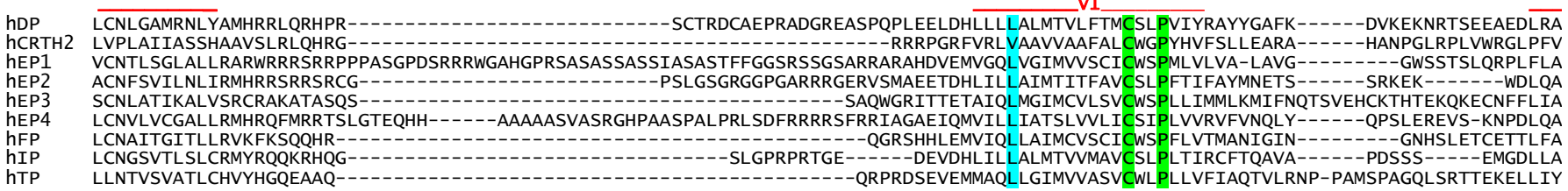

LLNTVSVATLCHVYHGQEAAQ

VII

hDP LRFLSVISIVDPWIFIIFRSPVFRIFFHKIFIRPLRYRSRCSNSTNMESSL

hCRTH2 TSLAFFNSVANPVLYVLTCPDMLRKLRRSLRTVLESVLVDDSELGGAGSSRRRRTSSTARSASPLALCSRPE-

hEP1 VRLASWNQILDPWVYILLRQAVLRQLLRLLPPRAGAKGGPAGLGLTPSAWEASSLRSSRHSGLSHF

hEP2 LRFLSINSIIDPWVFAILRPPVLRLMRSVLCCRISLRTQDATQTSCSTQSDASKQADL

hEP3 VRLASLNQILDPWVYLLLRKILLRKFCQVANAVSSCSNDGQKGQPISLSNEIIQTEA

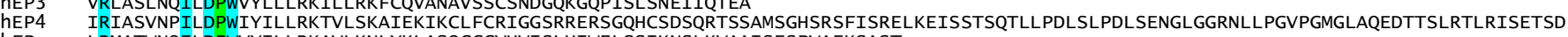

hFP LRMATWNQILDPWVYILLRKAVLKNLYKLASQCCGVHVISLHIWELSSIKNSLKVAAISESPVAEKSAST

$\begin{array}{ll}\text { hIP } & \text { FRFYAFNPILDPWVFILFRKAVFQRLKLWVCCLCLGPAHGDSQTPLSQLASGRRDPRAPS--- } \\ \text { hTP } & \text { LRVATWNQILDPWVYILFRRAVRRLQPRLSTRPSGVSLCGPAWSTVARSRLTATSASRVQAILVPQPPEQLGLA }\end{array}$

hCRTH2 ----EPRGPARLLGWLLGSCAASPQTGPLNRALSSTSS

hEP4 SSQGQDSESVLLVDEAGGSGRAGPAPKGSSLQVTFPSETLNLSEKCI

hIP APVGKEGSCVPLSAWGEGQVEPLPPTOQSSGSAVGTSSKAEASVACSLC

FIG. 1. Deduced amino sequence of human prostanoid receptors, in alignment. Amino acid residues with $100 \%$ homology between all receptors are highlighted in green. Amino acid residues with complete homology, except for $\mathrm{DP}_{2}$, are highlighted in blue.

Replacement of the corresponding serine by mutation to alanine had little effect on $\mathrm{PGD}_{2}$ binding (Hata et al., 2005). In contrast, replacement of lysine 209 in the fifth membrane-spanning domain by alanine greatly reduced $\mathrm{PGD}_{2}$ binding to $\mathrm{DP}_{2}$ receptors. Interaction of the carboxylate of $\mathrm{PGD}_{2}$ with Lys-209 would place $\mathrm{PGD}_{2}$ in an opposite orientation in the binding pocket to that proposed for other prostanoid receptors (Hata et al., 2005).

3. Second Messenger Signaling. Early signal transduction studies and a paucity of receptor selective ligands provided little insight into the pharmacology of prostanoid-sensitive receptors. The cloning of each prostanoid receptor and their transfection into and expression in cultured cells led to rapid elucidation of their $G$ protein coupling characteristics, at least with respect to

TABLE 1

Primary G protein coupling for prostanoid receptors

\begin{tabular}{cccl}
\hline $\begin{array}{c}\text { Prostanoid } \\
\text { Receptor } \\
\text { Subtype }\end{array}$ & $\begin{array}{c}\text { Prostanoid } \\
\text { Molecular Evolution } \\
\text { Cluster }\end{array}$ & G Protein & Second Messenger \\
\hline $\mathrm{DP}_{1}$ & 1 & $\mathrm{G}_{\mathrm{s}}$ & $\uparrow \mathrm{cAMP}$ \\
$\mathrm{EP}_{1}$ & 2 & $\mathrm{G}_{\mathrm{s}}$ & $\uparrow\left[\mathrm{Ca}^{2+}\right]_{\mathrm{i}}$ \\
$\mathrm{EP}_{2}$ & 1 & $\mathrm{GAMP}_{\mathrm{s}}$ & $\downarrow \mathrm{cAMP}$ \\
$\mathrm{EP}_{3}$ & 3 & $\mathrm{G}_{\mathrm{i}}$ & $\uparrow \mathrm{cAMP}$ \\
$\mathrm{EP}_{4}$ & 1 & $\mathrm{G}_{\mathrm{s}}$ & $\uparrow\left[\mathrm{Ca}^{2+}\right]_{\mathrm{i}}$ PI turnover \\
$\mathrm{FP}$ & 2 & $\mathrm{G}_{\mathrm{q}}$ & $\uparrow \mathrm{cAMP}$ \\
$\mathrm{IP}$ & 1 & $\mathrm{G}_{\mathrm{s}}$ & $\uparrow\left[\mathrm{Ca}^{2+}\right]_{\mathrm{i}}$ PI turnover \\
$\mathrm{TP}$ & 2 & $\mathrm{G}_{\mathrm{q}}$ & $\uparrow\left[{ }^{2}\right.$ \\
\hline
\end{tabular}

$\mathrm{G}_{\mathrm{q}}, \mathrm{G}_{\mathrm{s}}$, and $\mathrm{G}_{\mathrm{i}} \cdot \mathrm{DP}_{(1)}, \mathrm{EP}_{2}, \mathrm{EP}_{4}$, and IP receptors were classified as $\mathrm{G}_{\mathrm{s}}$-coupled; $\mathrm{EP}_{1}, \mathrm{FP}$, and TP receptors were designated as $\mathrm{G}_{\mathrm{q}}$-coupled; $\mathrm{EP}_{3}$ receptors seemed capable of coupling to both $\mathrm{G}_{\mathrm{q}}$ and $\mathrm{G}_{\mathrm{i}}$ (Coleman et al., 1994b). During the past decade and a half, the $\mathrm{DP}_{2}(\mathrm{CRTh} 2)$ receptor was discovered as a second $\mathrm{G}_{\mathrm{i}}$-coupled receptor. These findings are summarized in Table 1 . The signaling cascades associated with prostanoid receptor stimulation have been further investigated to reveal diverse pathways.

4. Agonist and Antagonist Drugs. The pharmacological characterization of prostanoid receptors was built on isolated tissue and cultured cell studies (Coleman et al., 1984). Despite an inherent reliance on pharmacological intuition and deduction and on low-throughput assays, highly selective ligands were obtained for certain receptors. 5-(6-Carboxyhexyl)-1-(3-cyclohexyl-3-hydroxypropyl)hydantoin (BW 245C) and 3-(3-[1,1'-biphenyl]-4-yl3-hydroxypropyl)-2,5-dioxo-4-imidazolidineheptanoic acid, ethyl ester (BW A868C) (Giles et al., 1989) were discovered as a selective agonist and antagonist, respectively, for $\mathrm{DP}_{1}$ receptors, previously designated DP receptors. trans-2-(4-(1-Hydroxyhexyl)phenyl)-5-oxocyclopentaneheptanoic acid (AH 13205) (Nials et al., 1993) and butaprost (Gardiner, 1986) were selective $\mathrm{EP}_{2}$ agonists and (4-benzamidophenyl)-(Z)-7-[(1R,2R,3R)-3- 
hydroxy-2-[(2R)-2-hydroxy-3-phenoxypropoxy]-5-oxocyclopentyl]hept-5-enoate (GR 63799X) was a potent and selective $\mathrm{EP}_{3}$ agonist (Bunce et al., 1991). Fluprostenol (Dukes et al., 1974; Coleman et al., 1984) and 17-phenyl $\mathrm{PGF}_{2 \alpha}$ (Woodward et al., 1995a) were known as selective FP agonists and, ultimately, formed the structural platform for launching antiglaucoma drugs. Above all, several potent and selective TP receptor antagonists were invented based on their potential utility for treating cardiovascular disease. Indeed, S-145 was sufficiently potent and selective to enable isolation of the TP receptor protein (Ushikubi et al., 1989; Hirata et al., 1991).

\section{B. Summary of New Developments}

1. Prostaglandin $D_{2}$ (CRTh2) Receptors. The eight prostanoid receptors described by the pharmacologybased classification were rapidly discovered by homologybased screening after structural identification of TP receptors. This made it unlikely that further similar receptors would emerge, and this has proved to be the case. More recently discovered prostanoid receptors are quite different. A second $\mathrm{PGD}_{2}$-sensitive receptor was long suggested by functional studies but, when discovered, was found to be structurally quite distinct (Hirai et al., 2001). The $\mathrm{DP}_{2}$ receptor, also and originally designated (CRTh2), is more closely related to chemo-attractant receptors. The $\mathrm{DP}_{2}$ receptor mediates effects that are opposed to those produced by $\mathrm{DP}_{1}$ receptor stimulation in some instances. Given the lack of structural identity between $\mathrm{DP}_{1}$ and $\mathrm{DP}_{2}(\mathrm{CRTh} 2)$ receptors, it is not surprising that ligand recognition can markedly diverge. For example, the cyclooxygenase (COX) inhibitor indomethacin actually stimulates $\mathrm{DP}_{2}(\mathrm{CRTh} 2)$ receptors (Hirai et al., 2002; Stubbs et al., 2002), albeit weakly.

2. Prostanoid Receptor Heterodimerization. The discovery that $G$ protein-coupled receptors may heterodimerize has explained certain pharmacological anomalies. Receptor heterodimerization offers the option of creating novel binding sites without evolution of a dedicated encoding gene. It also provides a means of closely regulating the activity of local hormones simultaneously released by intimately combining their receptors. IP/TP receptor heterodimerization provides an example of both phenomena: 1) a recognition site for 8-epi-PGE $\mathrm{PG}_{2}$ is created and 2) the pathological effects of TP are limited because cAMP levels are increased by $\mathrm{TxA}_{2}$ activating the associated heterodimeric protein (Wilson et al., 2004).

The pharmacology of $\mathrm{PGF}_{2 \alpha}$-ethanolamide (prostamide $\mathrm{F}_{2 \alpha}$ ), and its analog bimaprost, was elucidated by a longer and more traditional route. Numerous agonist studies suggested a pharmacological identity distinct from the classic prostanoid FP receptor; this was confirmed by the eventual discovery of selective antagonists (Woodward et al., 2008). All this led to the same place in that the bimatoprost recognition site was modeled by cotransfecting the wild-type FP receptor and an alternative mRNA splicing variant thereof (Liang et al., 2008).
3. Gene Deletion Studies. Gene deletion studies with mice lacking each of the individual prostanoid receptors have enabled further elucidation of prostanoids in health and disease. Moreover, they have revealed important functions that had not been previously appreciated. The roles of prostanoids in inflammation and immune regulation provide a good example. It is now clearer that prostanoids exert both pro-inflammatory and anti-inflammatory effects and regulate gene expression in mesenchymal and epithelial cells at inflammatory sites (Narumiya, 2009). This self-regulatory role, with prostanoids exerting a dual role in a singular process, is often seen in immunology-based reactions. It had been held that prostanoids exerted very little role in immunity, but gene deletion studies revealed that prostanoids work at many levels in immune responses (Narumiya, 2003). A striking example of the contribution of gene deletion is the discovery of $\mathrm{EP}_{1}$ receptor involvement in suppressing impulsive behavior in response to stress (Furuyashiki and Narumiya, 2009). These findings will provide novel direction from discovering additional prostanoid-based therapeutics.

Gene deletion studies are an important step forward in elucidating the role of prostanoids in physiology and pathophysiology, especially when viewed in the context of small-molecule-based research. There are potential pitfalls in using small molecules to determine the pharmacological basis of experimental disease and thereby to discover new therapeutic approaches. False-negative results may occur by virtue of inadequate bioavailability at the target tissue: metabolic disposition and pharmacokinetics are traditionally a terminal step in the drug discovery process that occurs before clinical evaluation. Given the very high attrition rate in drug discovery, false-positive animal model data are clearly commonplace. Seemingly beneficial effects in animal models of human disease may be misleading for several reasons. Off-target pharmacology can never be fully assessed, even by screens aimed at 400 to 500 targets. Druginduced toxicity is rarely overt in acute animal models but is likely to produce misleading positive effects, certainly in pain and inflammation models. Indications of toxicity could be obtained from such living animal studies but this rarely seems to be the case. Blood pressure and heart rate could readily be monitored by tail cuff instrumentation. Circulating leukocyte viability could be determined by in vitro studies on inflammatory cells that routinely determine cell viability. Studies employing genetically modified mice are more reliable in that there can be confidence that there is 1) no hidden offtarget pharmacology, 2) no toxicity of unknown origin, and 3) no bioavailability issue. Thus, the use of genetically modified mice provides a solution to certain drug discovery complications; species differences and potential compensatory mechanisms remain a concern. Finally, the choice of disease models to complement gene deletion studies is also an important consideration. 
Many animal models, for example LPS-induced uveitis (Caspi, 2006), seem to be models in search of a human disease to mimic.

4. New Agonists and Antagonists. The introduction of recombinant receptor technologies permitted highthroughput assays. This has resulted in selective and potent antagonists for all known prostanoid receptors, with the exception of $\mathrm{EP}_{2}$. Potent and selective agonists have now been discovered for all prostanoid receptors, with the possible exception of $\mathrm{EP}_{1}$. These pharmacological tools, with information provided by gene deletion studies, will result in therapies based on informed modulation of prostanoid-mediated events. Prostanoid-based therapeutics will realize its full potential.

Parallel to fitting ligands to known receptors, unexplained pharmacological characteristics associated with certain prostanoids have been successfully studied. The activities of certain "orphan" prostanoids, although not actually described as such, are now understood. The surprisingly high potency of the $\mathrm{PGD}_{2}$ metabolites 13,14-dihydro 15-keto $\mathrm{PGD}_{2}$ (Jones, 1976a,b; Jones and Wilson, 1978) and $\mathrm{PGJ}_{2}$ (Woodward et al., 1990) was explained by the discovery of the $\mathrm{DP}_{2}$ (CRTh2) receptor. Heterodimerization between IP and TP receptors provides a site for interaction with 8-epi- $\mathrm{PGE}_{2}$ (Wilson et al., 2004). Likewise, PG-ethanolamides, and their analog bimatoprost, have a predilection for cells cotransfected with wild type and an alternative mRNA splicing variant of the FP receptor (Liang et al., 2008).

5. Cell Signaling. Elucidation of the cell signaling pathways associated with prostanoid receptors has provided an additional dimension to the body of information required to conceptualize therapeutic applications. In many instances, the repertoire of signaling pathways is now known to be quite expansive for some receptors. These will be described for each individual prostanoid receptor.

\section{Receptor Types, Subtypes, and mRNA Splicing Variants}

\section{A. $D P_{1}$ Receptors}

1. Second Messenger Signaling. $\mathrm{DP}_{1}$ receptors are $\mathrm{G}_{\mathrm{s}}$-coupled and stimulate cAMP formation (Gorman et al., 1977; Whittle et al., 1978; Schafer et al., 1979; Halushka et al., 1989; Goh and Nakajima, 1990; Hirata et al., 1994; Boie et al., 1995). Cells expressing $\mathrm{DP}_{1}$ receptors also elicit an increase in $\left[\mathrm{Ca}^{2+}\right]_{\mathrm{i}}$ when stimulated by $\mathrm{PGD}_{2}$ or the selective $\mathrm{DP}_{1}$ agonist $\mathrm{BW} 245 \mathrm{C}$, but this is cAMP-dependent (Boie et al., 1995). This increase in $\left[\mathrm{Ca}^{2+}\right]_{\mathrm{i}}$ may result from activation of protein kinase $\mathrm{A}$ (PKA) and subsequent involvement of L-type $\mathrm{Ca}^{2+}$ channels and the ryanodine receptor (Zaccolo, 2009). This seems to be a minor pathway for transducing $\mathrm{DP}_{1}$ receptor signaling, cAMP/PKA activation being almost invariably involved. No evidence for the involvement of exchange proteins activated by cAMP (Epacs) seems to have emerged.
2. Distribution and Biological Functions. Among the classic prostanoid receptors, $\mathrm{DP}_{1}$ is the least abundant in tissues and exhibits a relatively narrow distribution. Northern blot analyses detected expression only in the retina and small intestine (Boie et al., 1995). In mice, $\mathrm{DP}_{1}$ receptor expression is moderate in the ileum; weak in the stomach, lung, and uterus; and absent elsewhere (Hirata et al., 1994). Functional studies, however, have described $\mathrm{DP}_{1}$ receptors present in certain cells. In some cases, transcripts have provided supportive data. Human platelets express functional $\mathrm{DP}_{1}$ receptors, which inhibit aggregation (Whittle et al., 1983; Giles et al., 1989; Trist et al., 1989; Seiler et al., 1990). Effects of $\mathrm{PGD}_{2}$ on vascular smooth muscle vary according to species (Giles and Leff, 1988). In humans, effects were restricted to facial flushing and nasal congestion (Heavey et al., 1984), with no meaningful alteration in blood pressure. Similar effects were apparent for the $\mathrm{DP}_{1}$ agonist BW 245C (Giles and Leff, 1988). Thus, despite evidence for presynaptic DP receptors that would enhance norepinephrine release (Molderings et al., 1994), this does not seem to translate into gross cardiovascular events. In contrast, the skin flushing associated with nicotinic acid used for treating dyslipidemia is $\mathrm{DP}_{1}$ receptor-mediated (Cheng et al., 2006).

Compelling pharmacological evidence for $\mathrm{DP}_{1}$ receptors in the human myometrium has been advanced (Senior et al., 1992; Fernandes and Crankshaw, 1995). $\mathrm{DP}_{1}$ receptor stimulation would mediate tocolysis. It is noteworthy that Northern blotting did not identify a $\mathrm{DP}_{1}$ receptor transcript in the human uterus (Boie et al., 1995). There are additional examples of $\mathrm{DP}_{1}$ receptors being identified in specific cell types or localized regions in which Northern blot analyses of tissue would suggest otherwise.

The initial studies on $\mathrm{DP}_{1}$ receptor distribution showed undectable to very low levels in the brains of both mice and humans (Hirata et al., 1994; Boie et al., 1995). Because $\mathrm{PGD}_{2}$ has well documented activity in the CNS, these data were interpreted to mean that expression was limited to certain areas and/or specific cells (Narumiya et al., 1999). This has proven correct, when subject to careful examination, for not only the brain but also other tissues (Gerashchenko et al., 1998; Wright et al., 1998). A pharmacological rationale for the various central effects of $\mathrm{PGD}_{2}$ and its analogs is therefore tenable. These effects include sleep regulation (Urade and Hayaishi, 1999), neuroprotection (Liang et al., 2005b; Saleem et al., 2007b; Thura et al., 2009) allodynia (Minami et al., 1997), and hyperalgesia (Telleria-Diaz et al., 2008). $\mathrm{DP}_{1}$ receptor involvement in neurotransmission is not limited to the CNS, and a local effect on pruritus has been reported (Arai et al., 2004, 2007; Sugimoto et al., 2007). The pruritic activity associated with scratching, in an atopic dermatitis-like model in NC/Nga mice, was inhibited by $\mathrm{PGD}_{2}$ but not metabolites known to stimulate $\mathrm{DP}_{2}$ receptors (Arai et 
al., 2004). These effects on experimental pruritus were confirmed using a $\mathrm{DP}_{1}$ agonist, $(4-((1 R, 2 S, 3 R, 5 R)-5$ chloro-2-((S)-3-cyclohexyl-3-hydroxyprop-1-ynyl)-3hydroxycyclopentyl)butylthio) acetic acid monohydrate (TS-022), and the antagonist BW A868C to establish the pharmacological basis of the attenuated itch response (Arai et al., 2007). In addition, accelerated repair of the disrupted cutaneous barrier was reported for TS022 (Arai et al., 2007), an effect ascribed not only to $\mathrm{DP}_{1}$ but also to $\mathrm{EP}_{3}$ and $\mathrm{EP}_{4}$ receptors in mice (Honma et al., 2005). A physiological role for $\mathrm{PGD}_{2}$ has also been contemplated, in that $\mathrm{PGD}_{2}$ released by mechanical scratching may be autoinhibitory, limiting the extent of the scratching response and preventing skin damage (Sugimoto et al., 2007; Takaoka et al., 2007). The NC/ Nga model may, at least in part, be operational with respect to the antipruritic effects of $\mathrm{DP}_{1}$ agonists by virtue of a reduced level of endogenous $\mathrm{PGD}_{2}$ production (Takaoka et al., 2007). The decrease in $\mathrm{PGD}_{2}$ production in the late phase of dermatitis and scratching in NC/Nga mice, together with increased $\mathrm{DP}_{1}$ receptor expression assists in explaining the potent antipruritic activity of TS-022 (Sugimoto et al., 2007). Related to atopic dermatitis, $\mathrm{DP}_{1}$ receptor stimulation impedes $\mathrm{TNF}$ - $\alpha$-induced migration of human Langerhans cells (LCs) and additional chemotactic events, which strongly decreases the recruitment of inflammatory cells in a model of murine atopic dermatitis (Angeli et al., 2004). The beneficial effects of TS-022 and BW 245C cannot be attributed solely to amelioration of pruritus by scratching behavior.

A study in mice also revealed that $\mathrm{DP}_{1}$ receptor stimulation inhibited airway inflammation and suppressed asthma by modulating dendritic cells and inducing regulatory $\mathrm{T}$ cells (Hammad et al., 2007). $\mathrm{PGD}_{2}$ also affects human dendritic cell differentiation and modulates the pattern of immunoregulatory cytokine production, favoring naive $\mathrm{T}$ cells toward a Th2 phenotype (Gosset et al., 2005). $\mathrm{PGD}_{2}$ may contribute to the control of allergic reactions and tumor formation (Gosset et al., 2005; Torres et al., 2008).

$\mathrm{DP}_{1}$ antagonists have also been the subject of antiinflammatory studies. $\mathrm{PGD}_{2}$ is the major prostanoid produced by mast cells; this presents an attractive target for DP-receptor drug design. Despite the evidence for $\mathrm{DP}_{1}$ receptor-mediated pathological increases in blood flow and engorgement of blood vessels in the nasal mucosa, clinical trials on the $\mathrm{DP}_{1}$ antagonist laropiprant demonstrated no efficacy in patients with allergic rhinitis or asthma (Philip et al., 2009). For therapeutic modalities based on attenuating the activity of $\mathrm{PGD}_{2}$, consideration of $\mathrm{DP}_{2}$ (CRTh2)-mediated events is probably of greater importance. The significance of $\mathrm{DP}_{1}$ receptor activation in inflammation and immune responses is best appreciated when considered in the context of $\mathrm{DP}_{2}$ (CRTh2) receptors.

$\mathrm{DP}_{1}$ receptor expression is high in the retina (Boie et al., 1995), but it could be argued that this finding has not been followed up to its full extent. $\mathrm{PGD}_{2}$ induces heme oxygenase- 1 expression in the retinal pigmented epithelium, an enzyme important for photoreceptor survival (Satarug et al., 2008). $\mathrm{DP}_{1}$ receptors exerted only a marginal influence on heme oxygenase-1 expression, the $\mathrm{DP}_{2}$ (CRTh2) receptor being important (Satarug et al., 2008). In addition to the retina, DP receptor expression occurs in the iris and ciliary body (Gerashchenko et al., 1998). Expression in the ciliary body entirely correlates with the ocular hypotensive activity of $\mathrm{DP}_{1}$ agonists and their mechanism of action (i.e., increased uveoscleral outflow) (Toris et al., 2006). $\mathrm{DP}_{1}$ receptor mRNA is also located in mucus-secreting goblet cells and the columnar epithelium of the rat gastrointestinal tract (Wright et al., 1998), but this does not transition into the eye, where goblet cell secretion is attributable to $\mathrm{DP}_{2}$ receptor pharmacology (Woodward et al., 1990, 1993b). Likewise, the $\mathrm{DP}_{1}$ receptor has been implicated in eosinophil trafficking (Schratl et al., 2007), but this was not observed in ocular studies (Woodward et al., 1990, 1993b). The most prominent effect of $\mathrm{DP}_{1}$ receptor stimulation in the eye is on intraocular pressure (IOP) (Goh et al. 1988; Nakajima et al., 1991; Woodward et al., 1993b). $\mathrm{DP}_{1}$ effects are summarized in Table 2 in the therapeutics section.

3. Gene Deletion Studies. One line of DP $\left(\mathrm{DP}_{1}\right)$-deficient mice has been generated (Matsuoka et al., 2000). Using this line of mice, physiological roles of $\mathrm{DP}_{1}$ in allergy and immunity, sleep induction and other brain functions, and tumor progression and angiogenesis have been examined. Given that $\mathrm{PGD}_{2}$ is a major PG produced by mast cells and released in large amount after antigen challenge, Matsuoka et al. (2000) examined its role in allergic inflammation using ovalbumin (OVA)induced asthma model. Sensitization and aerosol challenge of $\mathrm{DP}(-/-)$ mice with OVA-induced increases in the serum concentration of IgE similar to those observed in wild-type mice. However, the $\mathrm{DP}(-/-)$ animals developed substantially reduced asthmatic responses in this model; the concentrations of Th2 cytokines and the extents of lymphocyte accumulation and eosinophil infiltration in the lungs of the mutant animals after OVA challenge were greatly reduced compared with those apparent in the wild type. These observations indicate that $\mathrm{PGD}_{2}$ serves as a mediator of asthmatic responses. The authors found that $\mathrm{DP}_{1}$ is induced in airway epithelial cells after the challenge and suggested that $\mathrm{PGD}_{2}$ acts on epithelial cells to induce various allergy-associated genes to facilitate inflammation. On the other hand, Angeli et al. (2001), studying Schistosoma mansoni infection in the skin, found that parasite-derived $\mathrm{PGD}_{2}$ acts at $\mathrm{DP}_{1}$ receptors in LCs to induce their retention in the epidermis. They further showed that this retention can be mimicked by administration of a $\mathrm{DP}_{1}$ agonist, BW $245 \mathrm{C}$, during local TNF- $\alpha$ treatment and that the retention of LCs in the skin by $S$. mansoni infection or by $\mathrm{BW} 245 \mathrm{C}$ was impaired in $\mathrm{DP}_{1}$-deficient 
TABLE 2

Potential therapeutic application of $\mathrm{DP}_{1}$ agonists

\begin{tabular}{|c|c|c|c|c|c|c|}
\hline $\mathrm{DP}_{1}$ Agonist & Route & Dose & Species & Experimental Model & Indication & Reference \\
\hline $\begin{array}{l}572 \mathrm{C} 85 \\
192 \mathrm{C} 86\end{array}$ & Topical & $2-250 \mu \mathrm{g}$ & Rabbit, cat & Intraocular pressure & Glaucoma & Matsugi et al., 1995 \\
\hline AL-6598 & Topical & $0.01 \%$ & Monkey & Intraocular pressure & Glaucoma & Toris et al., 2006 \\
\hline BW 245C & Intrathecal & $83 \mathrm{ng} / \mathrm{kg}\left(\mathrm{IC}_{50}\right)$ & Mouse & $\begin{array}{l}\text { Nociceptin-induced } \\
\text { allodynia }\end{array}$ & Chronic pain & Minami et al., 1997 \\
\hline BW 245C & Topical & $2.5 \mu \mathrm{g}$ & Rabbit & Intraocular pressure & Glaucoma & Goh et al., 1988 \\
\hline BW 245C & Topical & $50 \mu \mathrm{M}$ & Mouse & $\begin{array}{l}\text { Epicutaneous } \\
\text { sensitization to } \\
\text { OVA }\end{array}$ & Atopic dermatitis & Angeli et al., 2004 \\
\hline BW 245C & Intratracheal & $100 \mu \mathrm{M}$ & Mouse & OVA-alum asthma & Asthma & Hammad et al., 2007 \\
\hline BW 245C & & $1-1000 \mathrm{nM}$ & Rat & Cell/tissue culture & $\begin{array}{l}\text { Neurodegenerative } \\
\text { diseases }\end{array}$ & Liang et al., 2005b \\
\hline BW 245C & $\begin{array}{l}\text { In vitro }{ }^{+}(-/- \\
\left.\mathrm{DP}_{1} \text { mice }\right)\end{array}$ & $0.05-5 \mu \mathrm{M}$ & Mouse & $\begin{array}{l}\text { Ischemia-reperfusion } \\
\text { injury }\end{array}$ & $\begin{array}{l}\text { Cerebral ischemic } \\
\text { disorders }\end{array}$ & Saleem et al., 2007b \\
\hline BW 245C & Topical & $0.01-1 \%$ & Rabbit & Intraocular pressure & Glaucoma & Woodward et al., 1990 \\
\hline $\begin{array}{l}\text { BW } 245 \mathrm{C} \\
\text { AS } 702224\end{array}$ & Intracavernosal & $\begin{array}{l}\text { BW 245C, } 59 \mathrm{nM} \text {; AS } 702224 \text {, } \\
29 \mathrm{nM}\left(\mathrm{EC}_{50} \text { in isolated }\right. \\
\text { human tissue) }\end{array}$ & $\begin{array}{l}\text { Rabbit, rat, } \\
\text { human } \\
\text { cavernosal } \\
\text { tissue }\end{array}$ & Penile tumescence & $\begin{array}{l}\text { Erectile } \\
\quad \text { dysfunction }\end{array}$ & Brugger et al., 2008 \\
\hline SQ-27986 & Topical & $2.5-25 \mu \mathrm{g}$ & Rabbit & Intraocular pressure & Glaucoma & Woodward et al., 1993 \\
\hline SQ-27986 & Topical & $1-30 \mu \mathrm{g}$ & Monkey & Intraocular pressure & Glaucoma & Crawford et al., 1992 \\
\hline TS-022 & Topical & $2.5 \rightarrow 250 \mathrm{nM}$ & Mouse & NC/Nga mice & Atopic dermatitis & Arai et al., 2007 \\
\hline
\end{tabular}

AL-6598, 2-[[(2Z)-4-[(1R,2R,3R,5R)-5-chloro-2-[(3R)-3-cyclohexyl-3- hydroxypropyl]-3-hydroxycyclopentyl]-2-buten-1-yl]oxy]-1-methylethyl ester, acetic acid.

mice. In $\mathrm{DP}_{1}$-deficient mice, LCs migrated to draining lymph nodes and triggered a Th2 response (Hervé et al., 2003). Such inhibitory modulation of dendritic cell (DC) function by DP stimulation is also found in DCs in the lung. Hammad et al. (2007) found that inhalation of BW $245 \mathrm{C}$ in mice during OVA challenge attenuated the asthmatic response and that this inhibitory effect of $\mathrm{BW}$ $245 \mathrm{C}$ was dependent on $\mathrm{DP}_{1}$ expression by lung DCs. They suggested that this $\mathrm{DP}_{1}$-dependent modulation of lung DCs operates in the actual process of allergic inflammation in the OVA-induced asthma response, because chimeric mice with $\mathrm{DP}(-/-)$ hematopoietic cells exhibited an enhanced airway response. Therefore, there is both enhancement and attenuation of $\mathrm{PGD}_{2}$ $\mathrm{DP}_{1}$ signaling-dependent pathways by different types of cells in allergic inflammation, and the final outcome shown in the study by Matsuoka et al. (2000) seems to represent the net effects of these pathways. Given the sleep-inducing effect of $\mathrm{PGD}_{2}$, Mizoguchi et al. (2001) examined localization of $\mathrm{DP}_{1}$ in the brain and examined whether $\mathrm{DP}_{1}$ is involved in $\mathrm{PGD}_{2}$-mediated sleep. They found that $\mathrm{DP}_{1}$ in the brain is mainly localized in arachnoid trabecular cells in the leptomeninges of the basal forebrain, and that infusion of $\mathrm{PGD}_{2}$ into the subarachnoid space of this region increased the extracellular adenosine level and induces an increase in the amount of nonrapid eye movement sleep in wild-type mice and not in $\mathrm{DP}_{1}$-deficient mice. Although this study unequivocally demonstrated the involvement of $\mathrm{DP}_{1}$ in $\mathrm{PGD}_{2}-$ induced sleep, the baseline sleep-wake patterns were essentially identical between WT and $\mathrm{DP}(-/-)$ mice. These results suggest either that the $\mathrm{DP}_{1}$-mediated system may not be crucial for physiological sleep regulation or that some other system may effectively compensate for the loss of the DP system involved in the regulation of sleep. The same group of researchers ( $\mathrm{Qu}$ et al., 2006) reported that administration of $\mathrm{SeCl}_{4}$, as in patients with insomnia, inhibits $\mathrm{PGD}_{2}$ production and induces insomnia in WT mice but not in $\mathrm{DP}(-/-)$ mice. Qu et al. (2006) further showed that administration of a specific $\mathrm{DP}_{1}$ antagonist, ONO-4127Na $\left(\mathrm{p} A_{2}, 9.73\right.$ for human $\mathrm{DP}_{1}$ ) (Torisu et al. 2003a,b), markedly reduced the sleep period in rats ( $Q u$ et al., 2006). On the basis of these findings, they concluded that the $\mathrm{PGD}_{2}-\mathrm{DP}_{1}$ signaling is involved in regulation of physiological sleep. The importance of DP in the brain has also been studied from the viewpoint of neuroinflammation. Mohri et al. (2006) detected significantly higher $\mathrm{PGD}_{2}$ concentration in the brain of the genetic demyelination twitcher mouse and found that this was due to induction of hematopoietic type PGD synthase in the microglia of these mice. They further found that $\mathrm{DP}_{1}$ was induced on astroglia, and loss of $\mathrm{DP}_{1}$ impaired astrogliosis and demyelination and lessened clinical severity in this line of mice. The same group (Taniguchi et al., 2007) also examined the role of the $\mathrm{PGD}_{2}-\mathrm{DP}_{1}$ signaling in hypoxic ischemic encephalopathy in neonatal mice. They subjected 7-day old pups of WT mice or mice deficient in L-PGDS, HPGDS, both L-PGDS and HPGDS, DP 1 , or CRTh2 to left carotid artery ligation and hypoxia exposure and examined the infarct size. They found that the infarct size was significantly enhanced in mice deficient in both L-PGDS and HPGDS or mice deficient in $\mathrm{DP}_{1}$. Given the induction of $\mathrm{DP}_{1}$ in endothelial cells and more severe damage of endothelial cells in $\mathrm{DP}(-/-)$ mice, these authors suggested that the $\mathrm{PGD}_{2}-\mathrm{DP}_{1}$ signaling exerts a protective action against hypoxic ischemic injury by an action on endothelial cells. Finally, Murata et al. (2008) used $\mathrm{DP}(-/-)$ mice and reported that the $\mathrm{PGD}_{2}-\mathrm{DP}_{1}$ signaling is involved in suppression of tumor-associated angiogenesis and hyperpermeability. In this experiment, they implanted Lewis lung cancer cells onto the back of WT 
and $\mathrm{DP}(-/-)$ mice and found enhanced tumor growth in DP(-I-) mice. Furthermore, they found decreased apoptosis and increased angiogenesis and vascular leakage associated with tumors in $\mathrm{DP}_{1}(-/-)$ mice. Given the expression of $\mathrm{DP}_{1}$ in endothelial cells and attenuation of angiogenesis and permeability in model systems, they suggested that $\mathrm{PGD}_{2}-\mathrm{DP}_{1}$ signaling exerts inhibitory actions in tumor-associated angiogenesis and plasma leakage.

4. Agonists and Antagonists. As a general premise, position, type and configuration of oxygen substituents on the cyclopentane ring determines the receptor specificity of natural prostanoids, although no single prostanoid is truly specific. In addition, high agonist potency is generally dependent on a C1-carboxylate, trans geometry of the side-chains ( $8 \alpha, 12 \beta$ as conventionally drawn), and a $15 S$-15-hydroxy moiety. The recent work of Ungrin et al. (2001) on the human $\mathrm{EP}_{1}$ receptor illustrates these relationships well. There are, however, important deviations from this general picture, including the retention of high potency in C1-primary alcohol and 16hydroxy prostanoids. In addition, quite small alterations to the $\omega$ terminus often results in large changes in potency. For example, the $\mathrm{EP}_{1}$ binding affinity of $17 S, 20$ dimethyl-3,7-dithia $\mathrm{PGE}_{1}$ is 63 -fold higher than its $17 R$ epimer (Maruyama et al., 2002a). Modification of the $\omega$ chain has, therefore, been an attractive and successful strategy in searching for selective agonists, especially because the synthetic approach is usually ring construction $\rightarrow \alpha$-chain attachment $\rightarrow \omega$-chain attachment. Nonprostanoid agonists have also been described for $\mathrm{DP}_{2}$, $\mathrm{EP}_{2}, \mathrm{EP}_{3}$, and IP receptors.

Replacement of the $\omega$-pentyl terminus with a cyclohexyl moiety has been a popular strategy in $\mathrm{DP}_{1}$ agonist development. For example, the hydantoin ring analog BW 245C (Town et al., 1983; Whittle et al., 1983) is commonly used as a standard agonist; it exhibits minimal $\mathrm{DP}_{2}$ agonism (Monneret et al., 2001; YoshimuraUchiyama et al., 2004). Restricting the conformational mobility of the $\alpha$-chain in hydantoin-type analogs conserves $\mathrm{DP}_{1}$ agonist potency [e.g., BW-587C (Fig. 2); Barraclough et al., 1996], whereas the introduction of methyl, ethyl, and $n$-propyl groups at $\mathrm{N}^{10}$ progressively reduces efficacy (Leff and Giles, 1992); $\mathrm{N}^{10}$-benzyl substitution affords pure $\mathrm{DP}_{1}$ antagonism (BW A868C). Other potent $\mathrm{DP}_{1}$ agonists containing a 15-cyclohexyl group include RS-93520 (Fig. 2), a prostacyclin analog with completely inverted stereochemistry in the bicyclic ring (Alvarez et al., 1991; Crider et al., 1999) and SQ27986 (Fig. 2), a $\mathrm{PGH}_{2}$ analog with correspondingly inverted stereochemistry (see Fig. 2; Seiler et al., 1990). Although the $9 \beta$-chloro-11 $\alpha$-hydroxy ring system in ZK-

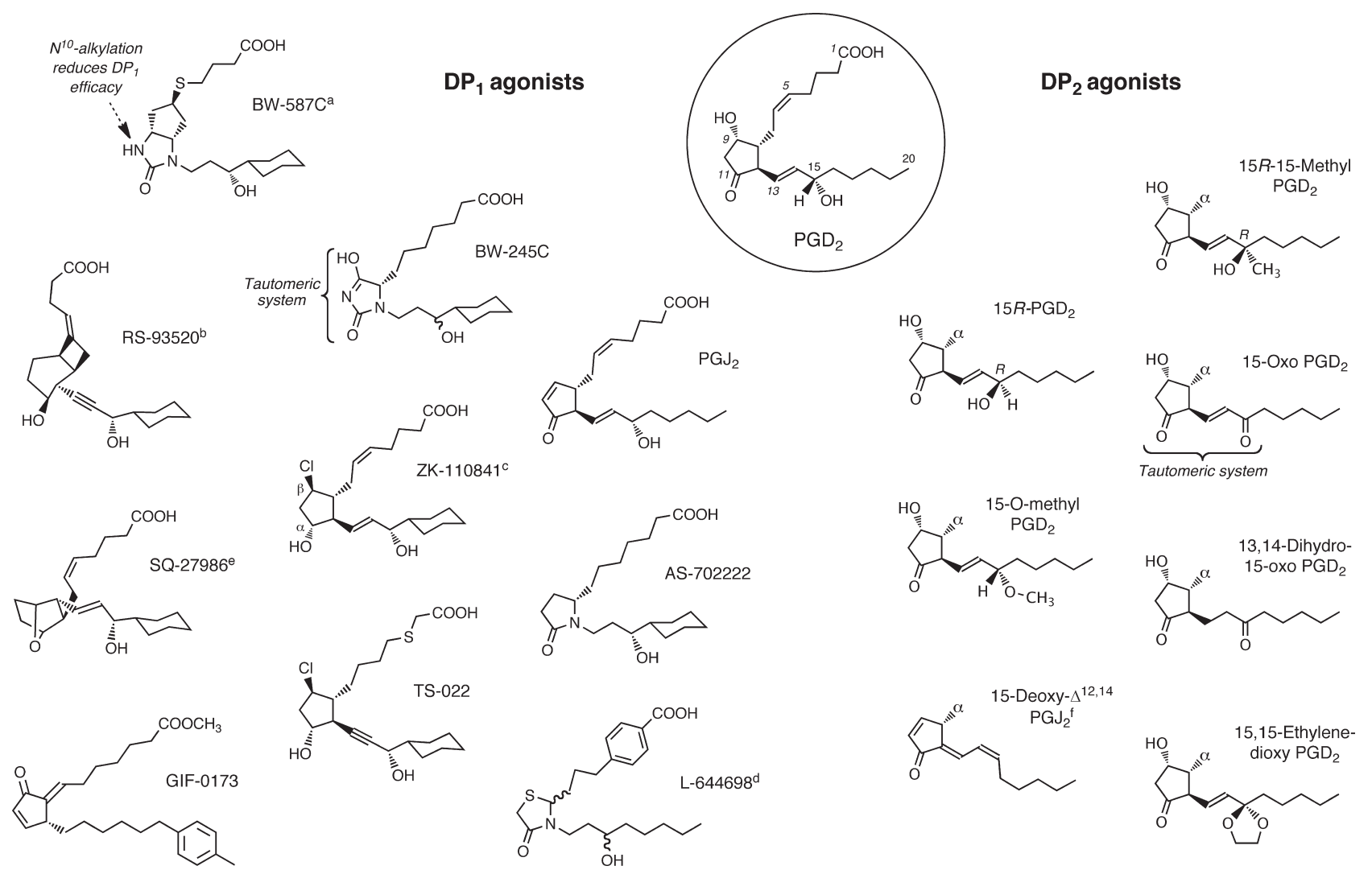

FIG. 2. Structures of agonists for prostanoid $\mathrm{DP}_{1}$ and $\mathrm{DP}_{2}$ receptors. $\mathrm{PGD}_{2}$, the most active natural agonist, is shown in the circle. Promotion of $\mathrm{DP}_{2}$-selectivity mainly involves alterations to $\mathrm{C} 15$; $\alpha$ indicates natural 2 -series side-chain. a ${ }^{\text {a }}$, ring system related to $6 \beta$-PGI ${ }_{1}{ }^{\text {b }}, \mathrm{PGI}_{2}$ analog with unnatural ring chirality. ${ }^{\mathrm{c}}, \mathrm{EP}_{1}$ and $\mathrm{EP}_{2}$ agonism retained. ${ }^{\mathrm{d}}$, some $\mathrm{EP}_{2}$ agonism remains. ${ }^{\mathrm{e}}, \mathrm{PGH}_{2}$ analog with unnatural ring chirality. ${ }^{\mathrm{f}}$, $14-c i s$ isomer is major component. 
110841 (Fig. 1) (Thierauch et al., 1988; Ney and Schrör, 1991) and its 3-oxa analog ZK-118182 (Darius et al., 1994) affords high $\mathrm{DP}_{1}$ agonism, high binding affinity for $\mathrm{EP}_{1}$ receptors is also found (Wright et al., 1998; Ungrin et al., 2001; Sharif and Davis, 2002). ZK-110841, ZK-118182, and the 13,14-dihydro analog of the latter [9-chloro-15-cyclohexyl-11,15-dihydroxy-3-oxa-16,17, 18,19,20-pentanor-5-prostenoic acid (AL-6556)] also seem to behave as partial agonists in human functional $\mathrm{EP}_{2}$ systems $\left(\mathrm{EC}_{50}, \sim 200-700 \mathrm{nM}\right.$ ) (Sharif et al., 2000, 2004); compare with structure of ONO-AE1-259 in Fig. 4.

Dehydration of the ring system of $\mathrm{PGD}_{2}$ to give $\mathrm{PGJ}_{2}$ results in retention of $\mathrm{DP}_{1}$ agonism (Fukushima et al., 1982; Mahmud et al., 1984; Wright et al., 1998) and approximately a 5 -fold loss of $\mathrm{DP}_{2}$ agonism (Monneret et al., 2002). The related 4-oxo-thiazolidine L-644698 (Fig. 2) shows high $\mathrm{DP}_{1} / \mathrm{DP}_{2}$ selectivity but still retains some affinity for $\mathrm{EP}_{2}$ receptors $\left(K_{\mathrm{i}}, 270 \mathrm{nM}\right.$; human recombinant) (Wright et al., 1998); indeed, complete elimination of $\mathrm{EP}_{2}$ agonism in the search for a selective $\mathrm{DP}_{1}$ agonist is not an easy task.

The first useful $\mathrm{DP}_{1}$ antagonist was the xanthonecarboxylic acid AH-6809 (Fig. 5) (Keery and Lumley, 1988). However, it has low affinity ( $\left.\mathrm{p} A_{2}, 5.9-6.6\right)$, shows considerable $\mathrm{EP}_{1}$ antagonism and also nonspecificity in the low micromolar range. Subsequently, BW A868C (Fig. 3), which is related to the $\mathrm{DP}_{1}$ agonist $\mathrm{BW} 245 \mathrm{C}$, emerged as a selective, competitive blocker of high affinity (human $\mathrm{DP}_{1}: \mathrm{p} A_{2},>9$ ) (Giles et al., 1989; Lydford et al., 1996); it is still a first choice antagonist.

The last 10 years has seen the development of other chemical classes of $\mathrm{DP}_{1}$ antagonist (Fig. 3) (Medina and Liu, 2006), driven by renewed interest in the pathogenic role of $\mathrm{PGD}_{2}$ in allergic disorders (see Pettipher, 2008). Emerging from bicyclic prostanoid analogs showing TP antagonism (Narisada et al., 1988), a pinane ring system with a sulfonamido linkage to an aromatic group led to high affinity, as in S-5751 (Fig. 3) (Arimura et al., 2001). A quite different approach has involved the use of indole-3-acetic acid, present in nonsteroidal anti-inflammatory drugs such as indomethacin, as a pharmacophore. In ONO-AE3-237 (Fig. 3), the acetate unit is located on $\mathrm{C} 4$; its binding $\mathrm{p} K_{\mathrm{i}}$ for the human $\mathrm{rc}-\mathrm{DP}_{1}$ receptor is 7.7 (Torisu et al., 2004). In the Merck series of $\mathrm{DP}_{1}$ antagonists, the positions of the carboxylic group and the substituents on the benzene ring of the indole template were optimized to yield MK-0524 (laropiprant) (Sturino et al., 2007). Laropiprant has very high affinity for the human $\mathrm{rc}-\mathrm{DP}_{1}$ receptor $\left(\mathrm{p} K_{\mathrm{i}}, 10.5\right)$; it also has $\sim 300$-fold lower affinity for the corresponding TP receptor, which requires consideration when using it to characterize prostanoid receptors.

5. Therapeutics. $\mathrm{DP}_{1}$ receptor agonists are not currently used therapeutically. $\mathrm{DP}_{1}$ agonists are not used to treat glaucoma because of the initial ocular hypertensive spike and an unacceptable incidence of ocular surface hyperemia (Nakajima et al., 1991). Perhaps the most promising of the newly advanced medical hypotheses is their potential utility in treating pruritus and atopic dermatitis (Angeli et al., 2004; Arai et al., 2004, 2007; Sugimoto et al., 2007). $\mathrm{DP}_{1}$ receptor antagonists, notably BW A868C, have been available for a long time (Giles et al., 1989). The report that laropiprant has no efficacy in patients with allergic rhinitis and asthma (Philip et al., 2009) is discouraging. It is likely that combined $\mathrm{DP}_{1} / \mathrm{DP}_{2}$ therapies offer greater promise for treating allergic diseases (Mitsumori, 2004; Pettipher, 2008; Jones et al., 2009). One therapeutic concept that emerged with a positive clinical outcome was the utility of a $\mathrm{DP}_{1}$ antagonist to limit the cardiovascular side effects of niacin. The investigational product Cordaptive was not approved as a drug in the United States but

\section{$\mathrm{DP}_{1}$ antagonists}

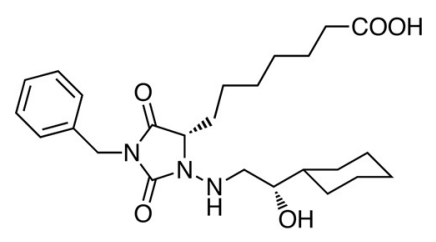

BW-A868C

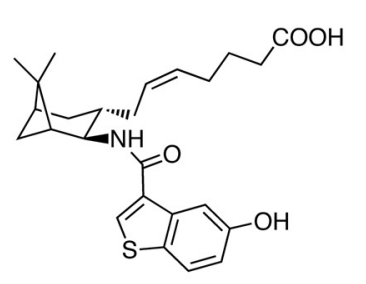

S-5751

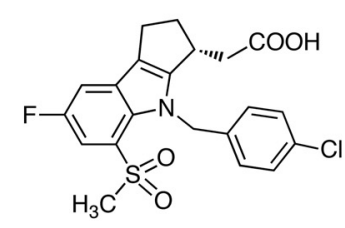

Laropiprant

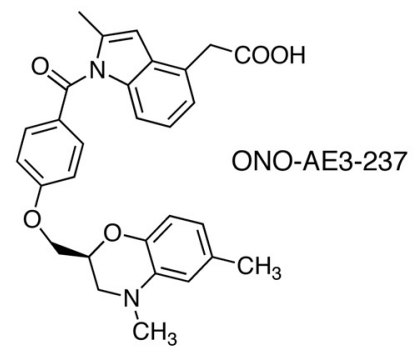

\section{$\mathrm{DP}_{2}$ antagonists}
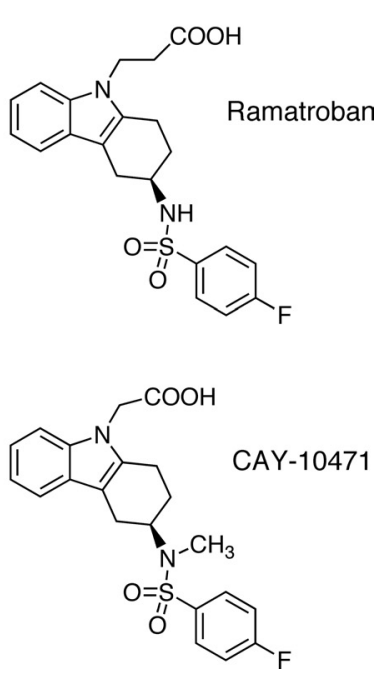

FIG. 3. Structures of representative $\mathrm{DP}_{1}$ and $\mathrm{DP}_{2}(\mathrm{CRTH} 2)$ antagonists. 
seems to have fared better as Tredaptive in Europe (Jones et al., 2009). Thus, the potent vasodilator effects of $\mathrm{DP}_{1}$ receptor stimulation discovered so many years ago (Coleman et al., 1994b) have successfully transitioned into treatment for nicotinic acid-induced flushing in patients with dyslipidemia (Cheng et al., 2006; Paolini et al., 2009). Finally, $\mathrm{DP}_{1}$ receptors have been implicated in the development of thyroid eye disease, which affects approximately $40 \%$ of patients with Graves hyperthyroidism. $\mathrm{DP}_{1}$ receptors were found to be an important factor in promoting hyaluronan production, which would be a major contribution to exophthalmos (Guo et al., 2010). Thus, laropiprant would be of potential value in this debilitating ophthalmic condition. The potential therapeutic utilities of $\mathrm{DP}_{1}$ receptor agonists and antagonists are summarized in Tables 2 and 3, respectively.

\section{B. Prostaglandin $E_{2}$ Receptors}

\section{EP ${ }_{1}$ Receptors.}

a. Second messenger signaling. The $\mathrm{EP}_{1}$ receptor has long been linked to $\mathrm{Ca}^{2+}$ mobilization, with a negligible PI response (Funk et al., 1993; Watabe et al., 1993; Katoh et al., 1995). $\mathrm{Ca}^{2+}$ mobilization patterns appear variable according to cell type studied. It is still not entirely clear which $\mathrm{G}$ protein(s) may be involved. The involvement of the phospholipase C (PLC)/PKC pathway in $\mathrm{EP}_{1}$-mediated trophoblast and osteoblast stimulation implies that $\mathrm{EP}_{1}$ receptors may couple to $\mathrm{G}_{\mathrm{q}}$ (Nicola et al., 2005; Tang et al., 2005). Compared with other prostanoid receptor studies, the signaling properties of $\mathrm{EP}_{1}$ receptors have received little attention. $\mathrm{EP}_{1}$ receptor mediated dephosphorylation of phosphatase and tensin homolog deleted on chromosome 10 and protein kinase B (Akt) (Zhou et al., 2008), and NO/cGMP pathway effects have been reported (Bachteeva et al., 2007).

The coexpression of more than one prostanoid receptor or isoform adds to the diversity of effects already inherent to prostanoid receptor stimulation. These include, but are not limited to, transactivation and crossdesensitization. $\mathrm{EP}_{1}$ receptor stimulation results in desensitization of TP receptors by PKC-mediated phosphorylation of C-terminal residues (Kelley-Hickie and Kinsella, 2004). An $\mathrm{EP}_{1}$ receptor-mediated transactivation of epidermal growth factor receptors, with resultant Akt activation, has also been reported (Han and $\mathrm{Wu}$, 2005). The potential for transactivation obviates the need to distinguish such an event from signaling cas- cades directly emanating from activation of $\mathrm{EP}_{1}$ receptors per se.

b. Distribution and biological functions. Contractile $\mathrm{EP}_{1}$ receptors do not have a widespread distribution in higher species and are more common in guinea pigs and murine species (Coleman et al., 1994b). In human tissues and cells, functional $\mathrm{EP}_{1}$ receptors have been demonstrated in the myometrium (Senior et al., 1991), pulmonary veins (Norel et al., 2004), mast cells (Wang and Lau, 2006) colonic longitudinal muscle (Smid and Svensson, 2009), and keratinocytes (Konger et al., 2009).

Northern blotting revealed $\mathrm{EP}_{1}$ transcription in the lung, stomach, and kidney of mice (Watabe et al., 1993). Functional studies in knockout mice and the employment of pharmacological tools have provided evidence for physiological participation for $\mathrm{EP}_{1}$ receptors in each of these organs. $\mathrm{EP}_{1}$ receptors produce airway constriction in mice, but this seems to be neuronally mediated rather than a direct smooth muscle effect (Tilley et al., 2003). $\mathrm{EP}_{1}$ receptors are also claimed to mediate $\mathrm{PGE}_{2}$ induced surfactant secretion from rat alveolar type II cells (Morsy et al., 2001). In the stomach, $\mathrm{EP}_{1}$ receptors seem to have detrimental and beneficial effects. Histamine-induced gastric injury, in the form of increased vascular permeability, is worsened by $\mathrm{EP}_{1}$ receptor activation (Hase et al., 2003). On the other hand, the $\mathrm{EP}_{1}$ receptor affords cytoprotection to the gastric mucosa against hemorrhagic lesions produced by indomethacin and $\mathrm{HCl} / \mathrm{C}_{2} \mathrm{H}_{5} \mathrm{OH}$ injury (Kunikata et al., 2001; Takeuchi et al., 2001a,c). $\mathrm{EP}_{1}$ receptors are essential for $\mathrm{HCO}_{3}^{-}$ secretion in response to mucosal acidification in the stomach (Takeuchi et al., 2006). A dual role for $\mathrm{EP}_{1}$ receptors in esophagitis is clearer, whereby $\mathrm{PGE}_{2}$ has a protective effect at low doses and a deleterious effect at high doses (Yamato et al., 2005). In the kidney, $\mathrm{PGE}_{2}$ activates $\mathrm{EP}_{1}$ receptors to inhibit $\mathrm{Na}^{+}$absorption by the renal collecting duct (Guan et al., 1998). $\mathrm{EP}_{1}$ receptors up-regulate transcription of the $\mathrm{Na}, \mathrm{K}-\mathrm{ATPase} \beta$ subunit in Madin-Darby canine kidney cells (Matlhagela and Taub, 2006) and attenuate up-regulation of epithelial sodium channel mRNA in inner medullary collecting duct cells by aldosterone (González et al., 2009). $\mathrm{EP}_{1}$ antagonists have been claimed as useful in the prevention of diabetic nephropathy (Makino et al., 2002) and hypertension-induced renal injury (Suganami et al., 2003). Severe renal impairment has been reported in glomerulonephritic $\mathrm{EP}_{1}$ knockout mice, underscoring an important role for $\mathrm{EP}_{1}$ in pathological renal conditions

TABLE 3

Potential therapeutic application $\mathrm{DP}_{1}$ antagonists

\begin{tabular}{|c|c|c|c|c|c|c|}
\hline $\begin{array}{c}\mathrm{DP}_{1} \\
\text { Antagonist }\end{array}$ & Route & Dose & Species & Experimental Model & Indication & Reference \\
\hline Laropiprant & Oral & $\begin{array}{l}37.5 \rightarrow 300 \mathrm{mg} \\
\quad(\text { human dose })\end{array}$ & Mice, humans & $\begin{array}{l}\text { NA-induced cutaneous } \\
\text { vasodilation in mice }\end{array}$ & $\begin{array}{l}\text { Adjunctive therapy } \\
\text { for dyslipidemia }\end{array}$ & $\begin{array}{l}\text { Cheng et al., 2006; } \\
\text { Paolini et al., 2009 }\end{array}$ \\
\hline S-5751 & Oral & $30 \mathrm{mg} / \mathrm{kg}$ & Sheep & $\begin{array}{l}\text { Ascaris suum-induced } \\
\text { asthma }\end{array}$ & asthma & Shichijo et al., 2009 \\
\hline
\end{tabular}

NA, nicotinic acid. 
(Rahal et al., 2006). Although a few arguably beneficial effects may be ascribed to $\mathrm{EP}_{1}$ receptor activation, most effects are pathophysiological.

Prevention of $\mathrm{EP}_{1}$ receptor activation has been purported as an attractive proposition for many diseases but colon cancer, systemic hypertension and, above all, inflammation and associated pain have received the most attention. Cyclooxygenases and their products have long been considered to play a role in colon carcinogenesis. Animal models have provided a direct link between $\mathrm{EP}_{1}$ receptors and colon cancer. Aberrant crypt foci, induced by azoxymethane, were reduced by the $\mathrm{EP}_{1}$ antagonists 6-((2S,3S)-3-(4-chloro-2-methylphenysulfonylaminomethyl)-bicyclo(2.2.2)octan-2-yl)-5Z-hexenoic acid (ONO-8711) (Watanabe et al., 2000; Kawamori et al., 2001a) and ONO-8713 (Fig. 5) (Watanabe et al., 2000). A reduction in intestinal polyps was observed in the adenomatous polyposis coli gene knockout mouse model of tumorigenesis (Watanabe et al., 1999; Kitamura et al., 2003b).

A role for $\mathrm{EP}_{1}$ receptor in cardiovascular homeostasis is indicated by knockout mouse studies (Audoly et al., 1999; Stock et al., 2001). Physiological regulation by presynaptic $\mathrm{EP}_{1}$ receptors has been recently implicated in nitrergic neurovascular transmission (Jadhav et al., 2009). In models of hypertension, blockade of $\mathrm{EP}_{1}$ receptors or gene deletion seems to confer antihypertensive effects in diabetic mice (Rutkai et al., 2009) and spontaneously hypertensive rats (Guan et al., 2007). $\mathrm{EP}_{1}$ antagonist treatment also dramatically improved arteriolar lesions (Suganami et al., 2003).

The involvement of $\mathrm{EP}_{1}$ receptors in inflammation, inflammatory pain and hyperalgesia, and neuropathic pain has been a major research focus. The antiinflammatory activity of $\mathrm{EP}_{1}$ antagonists has been reviewed extensively (Jones et al., 2009). $\mathrm{EP}_{1}$ receptors contribute to neuronal sensitization at peripheral sites (Omote et al., 2001) and at several levels in the CNS. $\mathrm{EP}_{1}$ receptors are localized in dorsal root ganglion neurons (Nakayama at el., 2004). Intrathecal $\mathrm{PGE}_{2}$ causes hyperalgesia in response to innocuous mechanical stimuli, an effect found to be $\mathrm{EP}_{1}$ receptor-mediated (Minami et al., 1994; Nakayama et al., 2004). Intrathecal administration of the $\mathrm{EP}_{1}$ antagonist ONO-8711 was shown to inhibit only the late phase of the mechanical hyperalgesic response associated with carrageenan-induced rat paw edema (Nakayama et al., 2002) and postoperative pain (Omote et al., 2002). These results correlate with a study on intra-articular Kaolin injection, in which spinal application of an $\mathrm{EP}_{1}$ agonist caused hyperexcitability 7 to $11 \mathrm{~h}$ after administration of the inflammatory stimulus (Bär et al., 2004). This time-dependent late phase response was not observed for $\mathrm{EP}_{2}$ and $\mathrm{EP}_{4}$ agonists (Bär et al., 2004). Set against these findings with respect to $\mathrm{EP}_{1}$ receptors in the spinal dorsal horn mediating hyperalgesia, microinjection of $\mathrm{PGE}_{2}$ into the ventromedial hypothalamus produced an $\mathrm{EP}_{1}$ receptor-mediated antinociceptive effect (Hosoi et al., 1999). Electrophysiological evidence has been provided for $\mathrm{EP}_{1}$-mediated hypoalgesia in response to noxious pinching of facial skin after lateral cerebroventricular administration of a receptor selective agonist and antagonists (Oka et al., 1997). These findings suggest that spinal processing of peripheral input may be subsequently relayed by $\mathrm{EP}_{1}$ receptors to higher centers, where the same $\left(\mathrm{EP}_{1}\right)$ receptors attenuate transmission. A strong, centrally mediated override by $\mathrm{EP}_{1}$ receptors does not, however, seem to be the case because systemically administered $\mathrm{EP}_{1}$ antagonists are widely reported to be analgesic and antiallodynic (Hall et al., 2007a; Jones et al., 2009).

The $\mathrm{EP}_{1}$ receptor plays additional significant roles in the CNS. Of considerable interest is the role of $\mathrm{EP}_{1}$ receptors in controlling stress-induced impulse behavior. Thus, in mice lacking $\mathrm{EP}_{1}$ receptors, stress induces impulsive aggression, an exaggerated acoustic startle response, impaired cliff avoidance, and social dysfunction (Matsuoka et al., 2005). This behavioral phenotype was reproduced in wild-type mice by an $\mathrm{EP}_{1}$ antagonist and corrected by a dopaminergic antagonist (Matsuoka et al., 2005), establishing a link between $\mathrm{EP}_{1}, \mathrm{DP}_{1}$, and $\mathrm{D}_{2}$ receptor function (Kitaoka et al., 2007). $\mathrm{EP}_{1}$ receptor stimulation has also been shown to cause hyperthermia (Oka and Hori, 1994; Oka et al., 2003b).

Beyond studies on tissues and living animals, $\mathrm{EP}_{1}$ expression and functional analyses in individual cell types has produced interesting results. In human primary keratinocytes, $\mathrm{EP}_{1}$ receptors evoked intracellular $\mathrm{Ca}^{2+}$ mobilization and were shown to be expressed in the epidermis (Konger et al., 2005a). The growth of malignant keratinocytes (Thompson et al., 2001) and regulation of keratinocyte differentiation (Konger et al., 2009) seem to be $\mathrm{EP}_{1}$ receptor-dependent. $\mathrm{EP}_{1}$ receptor stimulation caused differentiation of uncommitted $\mathrm{T}$ cells to a Th1 phenotype, which are involved in cellmediated immune reactions, such as dinitrofluorobenzene (DNFB) contact sensitivity (Nagamachi et al., 2007). Blockade of $\mathrm{EP}_{1}$ receptors has been shown to inhibit receptor activator of nuclear factor- $\kappa \mathrm{B}$ ligand (RANKL)-induced osteoclastogenesis (Tsujisawa et al., 2005). Hypoxia induces increased $\mathrm{EP}_{1}$ receptor expression in osteoclasts (Lee et al., 2007a) by a signal transduction pathway involving HIF- $1 \alpha$ (Genetos et al., 2009). A systematic study on $\mathrm{EP}_{1}$ receptor-mediated upregulation of HIF- $1 \alpha$ implicated $\mathrm{G}_{\mathrm{i}}$ coupling with activation of a PI3K/Akt/mammalian target of rapamycin signaling pathway, and HIF- $1 \alpha$ induction was associated with phosphorylation of the ribosomal protein 56 (Ji et al., 2010). Most noteworthy is perhaps the involvement of $\mathrm{EP}_{1}$ receptors in the proliferation of growth plate chondrocytes, the growth plate functioning to ossify long bones (Brochhausen et al., 2006).

c. Gene deletion studies. $\mathrm{EP}_{1}(-/-)$ mice appeared quite normal in all respects, including morphological characteristics (Ushikubi et al., 1998). Prostanoid EP 1 
receptors have long been heavily implicated in all aspects of nociception, from hyperalgesia to neuropathic pain. The advent of knockout mice has enabled follow-up of these observations, which were made with agonists and antagonists and the inherent complications of offtarget pharmacology, drug-induced toxicity, and substandard experimental design. A role for $\mathrm{EP}_{1}$ receptors in pain was supported by studies on the stretching/ writhing response to noxious chemical stimuli (Stock et al., 2001). Nevertheless, other $\mathrm{EP}_{1}$ gene deletion studies failed to confirm the substantive role of $\mathrm{EP}_{1}$ receptors in pain and inflammation suggested by agonist/antagonists studies in living animal models (Jones et al., 2009). According to gene deletion studies, IP receptors would be regarded as more important mediators of pain and inflammation (Murata et al., 1997; Ueno et al., 2000; Honda et al., 2006). Studies on $\mathrm{EP}_{1}(-/-)$ mice actually indicated an increase in thermal nociception, $\mathrm{EP}_{1}$ receptors being suggested to exert centrally mediated control of thermal pain sensitivity (Popp et al., 2009).

Perhaps the most intriguing aspect of CNS function that emerged from gene deletion studies is the role of $\mathrm{EP}_{1}$ receptors in regulating stress responses $(\mathrm{Fu}-$ ruyashiki and Narumiya, 2009). Stress, defined as a condition where body homeostasis is perturbed (Furuyashiki and Narumiya, 2009), elicits an adaptive response. This may take the form of febrile, neuroendocrine, and behavioral responses, and $\mathrm{EP}_{1}$ receptors participate in all of these. Although $\mathrm{EP}_{3}$ seems to be the dominant receptor in mediating fever (Ushikubi et al., 1998; Oka et al., 2003a; Furuyashiki and Narumiya, 2009), $\mathrm{EP}_{1}$ and $\mathrm{EP}_{3}$ receptors are equally important in $\mathrm{PGE}_{2}$-evoked adrenocorticotropic hormone and glucocorticoid release via the activation of corticotrophinreleasing hormone-containing neurons in the paraventricular nucleus of the hypothalamus (Matsuoka et al., 2003). The behavioral effects observed in $\mathrm{EP}_{1}(-/-)$ mice are notable. Social withdrawal, impulse aggression, impaired cliff avoidance, and an enhanced acoustic startle response were apparent (Matsuoka et al., 2005). These behaviors were attributed to a lack of inhibition of impulsive activity, implying that $\mathrm{EP}_{1}$ receptors suppress impulsive activity under stress (Matsuoka et al., 2005). The $\mathrm{EP}_{1}$ receptor is also neurotoxic and has been postulated as the essential downstream effector of COX-2induced neurocytotoxicity (Kawano et al., 2006). In $\mathrm{EP}_{1}$ receptor-deficient mice, it was found that COX-2-derived $\mathrm{PGE}_{2}$ does not mediate NMDA cytotoxicity (Kawano et al., 2006). $\mathrm{EP}_{1}$ receptor gene deletion also ameliorated brain injury produced by oxygen/glucose deprivation (Kawano et al., 2006) and ischemic damage produced by middle cerebral artery occlusion (Ahmad et al., 2006a; Kawano et al., 2006). In contemplating $\mathrm{EP}_{1}$ receptor participation in the middle cerebral artery occlusion model, it is important to take into consideration that cerebral blood flow is significantly increased in $\mathrm{EP}_{1}(-/-)$ mice (Saleem et al., 2007a). $\mathrm{EP}_{1}$ receptors have also been impli- cated in innate immune responses in the CNS. $\mathrm{PGE}_{2}$, signaling via either $\mathrm{EP}_{1}$ or $\mathrm{EP}_{2}$ receptors, is essential for Toll-like 4 receptor-mediated depletion of intermediate progenitor cells from the hippocampal subgranular zone (Keene et al., 2009).

A role for $\mathrm{EP}_{1}$ receptors in carcinogenesis has been confirmed by gene deletion studies. In the azoxymethane colon cancer model, formation of aberrant crypt foci was reduced by approximately $40 \%$ in $\mathrm{EP}_{1}(-/-)$ mice (Watanabe et al., 1999), and the number of tumors formed was essentially halved (Kawamori et al., 2005). In the methylcholanthrene-induced sarcoma model (MGC 101 mice), long-term growth was attenuated in $\mathrm{EP}_{1}$-deficient mice (Axelsson et al., 2005; Wang et al., 2005). The effects of cyclooxygenase inhibition on tumor progression were primarily on cell proliferation and apoptosis; angiogenesis was not an obvious primary determinant of onset and progression of tumor development (Axelsson et al., 2005).

Resting systolic blood pressure was reduced by approximately $10 \mathrm{~mm} \mathrm{Hg}$ in $\mathrm{EP}_{1}$ receptor-deficient mice (Stock et al., 2001). These findings transitioned into cardiovascular hypertension, where $\mathrm{EP}_{1}$ receptor disruption spared the protein kinase $\mathrm{N}$ locus and reduced the acute vasopressor response and chronic hypertension produced by angiotensin II (Guan et al., 2007). The hypotension in $\mathrm{EP}_{1}$-deficient mice, notably male mice, was reported to elicit physiological compensation that manifested as increased pulse rate, increased renin mRNA levels in the kidney, and increased plasma renin activity (Stock et al., 2001). In experimental glomerulonephritis, there was reduced urine osmolality, and overall renal damage was more acute in $\operatorname{EP}_{1}(-/-)$ mice (Rahal et al., 2006). $\mathrm{PGE}_{2}$, via $\mathrm{EP}_{1}$, modulates urine concentration not modulated in the renal collecting duct but within the hypothalamus to promote arginine vasopressin biosynthesis in response to water deprivation (Kennedy et al., 2007). In a model of bladder outlet obstruction, detrusor hyperactivity was negligible in $\mathrm{EP}_{1}$ receptor knockout mice (Schröder et al., 2004).

Two further findings from $\mathrm{EP}_{1}(-/-)$ mice are noteworthy: 1) $\mathrm{EP}_{1}$ receptors seem to be critically involved in shifting the Th1/Th2 balance to Th1 dominance (Nagamachi et al., 2007); this was demonstrated in a therapeutic sense by reduced DNFB-induced contact sensitivity in $\mathrm{EP}_{1}$ receptor-deficient mice (Nagamachi et al., 2007). 2) Adaptive gastric cytoprotection is apparently mediated by $\mathrm{EP}_{1}$ receptors (Takeuchi et al., 2001a,c).

d. Agonists and antagonists. 17-Phenyl $\mathrm{PGE}_{2}$ (Fig. 4) has modest $\mathrm{EP}_{1} / \mathrm{EP}_{3}$ selectivity (Lawrence et al., 1992) and is a useful agonist in Schild antagonism protocols because of its high potency. ONO-DI-004 (Fig. 4) is a more selective $\mathrm{EP}_{1}$ agonist (Okada et al., 2000; Suzawa et al., 2000), resulting from development of 6-oxo $\mathrm{PGE}_{1}$ via its 17S,20-dimethyl (methyl ester) analog [OU-1308 (ornoprostil)] (Kobayashi et al., 1991). 6a-Carba analogs of prostacyclin, such as carbacyclin and iloprost, are unex- 


\section{$E P_{1}$ agonists}
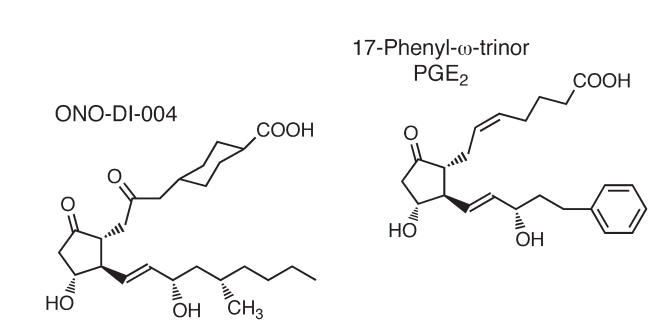

\section{$E P_{2}$ agonists}

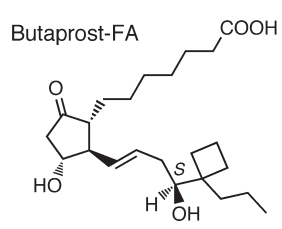

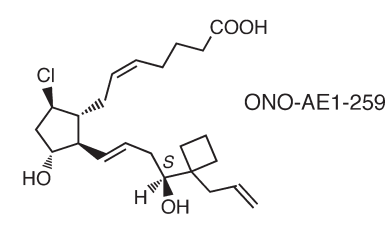

CP-533536
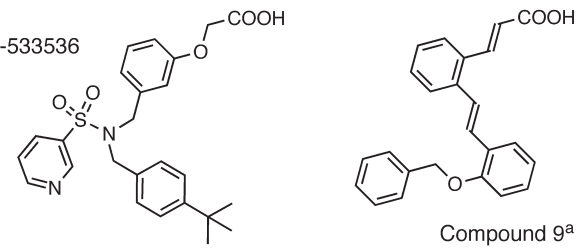

$\mathrm{EP}_{4}$ agonists

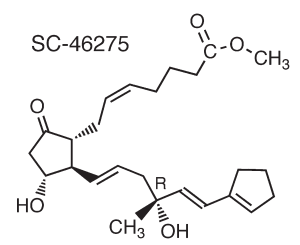

$E P_{3}$ agonists
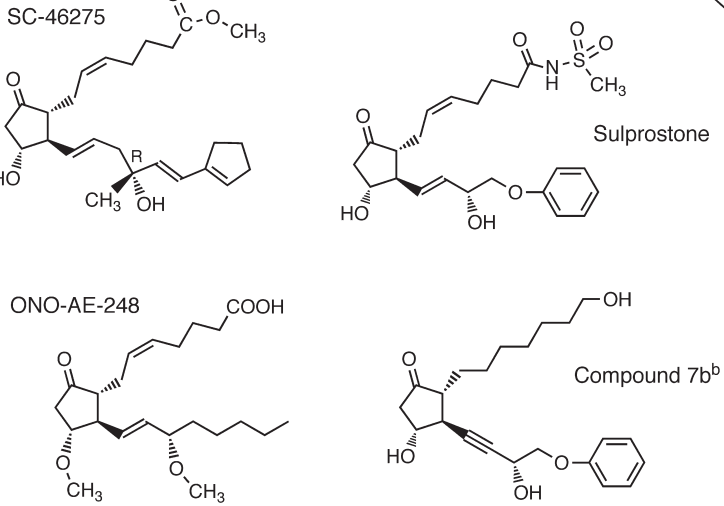

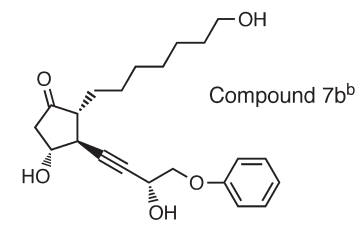

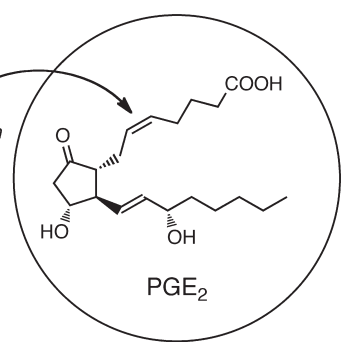

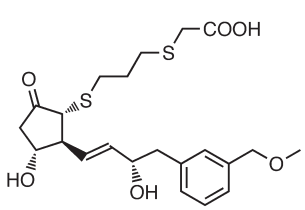

ONO-AE1-329

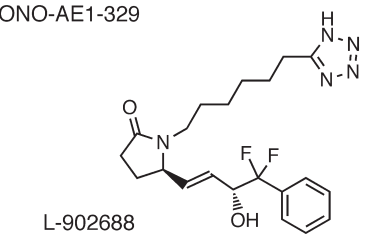

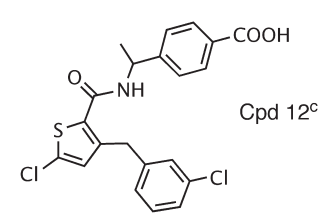

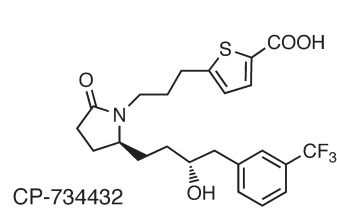

FIG. 4. Structures of agonists for prostanoid EP receptor subtypes. $\mathrm{PGE}_{2}$, the most active natural agonist, is shown in the circle. 17-Phenyl PGE and sulprostone have modest $\mathrm{EP}_{1} / \mathrm{EP}_{3}$ and $\mathrm{EP}_{3} / \mathrm{EP}_{1}$ selectivities, respectively. $\mathrm{CP}-533536$ and compound 9 are $\mathrm{EP}_{2}$ agonists with nonprostanoid structures. ${ }^{a}$, Belley et al. (2005). ${ }^{b}$, Shimazaki et al. (2000). ${ }^{\mathrm{c}}$, Blovin et al. (2010).

pectedly potent $\mathrm{EP}_{1}$ agonists (Dong and Jones, 1982; Dong et al., 1986; Lawrence et al., 1992), iloprost showing partial agonism in some systems (Dong and Jones, 1982; Dong et al., 1986; Boie et al., 1997). Functional studies with rat and human recombinant $\mathrm{EP}_{1}$ receptors in either reporter gene (Durocher et al., 2000) or aequorin-based $\mathrm{Ca}^{2+}$ flux assays (Boie et al., 1997; Ungrin et al., 2001) have confirmed and expanded these structure-activity relationship data.

The first $\mathrm{EP}_{1}$ receptor antagonist was 8-chlorodibenzo $(Z)[b, f][1,4]$ oxazepine-10 $(11 H)$-carboxylic acid, 2-acetylhydrazide (SC-19220), which is a dibenzoxazepine hydrazide (Sanner, 1969). It has low affinity $\left(\mathrm{p} A_{2}=5.5\right)$, but proved useful in the early characterization of EP receptor pharmacology. Modification of SC19220 , notably removal of the acetyl group, led to the thioether SC-51222 (Fig. 5), which was much more potent than the corresponding sulfone (Hallinan et al., 1994). Within the Searle series, SC-51322 has become the agent of choice for $\mathrm{EP}_{\mathbf{1}}$ receptor pharmacology studies (Fig. 5).

An alternative early $\mathrm{EP}_{1}$ antagonist was $\mathrm{AH}-6809$ (Fig. 5). Over a 0.1 to $10 \mu \mathrm{M}$ concentration range, it is selective for $\mathrm{EP}_{1}$ receptors $\left(\mathrm{p} A_{2}, 7.4\right)$ (Coleman et al., 1987; Eglen and Whiting, 1988; Lawrence et al., 1992) and does not block $\mathrm{EP}_{3}$ receptors (Lawrence et al., 1992;
Racké et al., 1992; Qian et al., 1994). AH-6809 was later reported to antagonize the human $\mathrm{EP}_{2}$ receptor (Woodward et al., 1995b) and is now probably more useful for this purpose, given the diverse array of potent and selective $\mathrm{EP}_{1}$ antagonists currently available.

$\mathrm{EP}_{1}$ antagonists designed by Ono Pharmaceuticals show an interesting progression from the TP antagonist $11 \alpha$-carba-12-(2'S-hydroxy-3' -phenylpropylamino)$9 \alpha, 11 \alpha$-isopropylideno- $\omega$-octanor-prost-5Z-enoic acid (ONO-11120) (Katsura et al., 1983) to a related pinane analog (ONO-NT-012; Minami et al., 1995) showing $\mathrm{EP}_{1}$, $\mathrm{FP}$, and TP antagonism (and $\mathrm{EP}_{3}$ agonism) to a bicyclo[2.2.2] octane analog (ONO-8711) showing $\mathrm{EP}_{1} /$ $\mathrm{EP}_{3}$ antagonism and ultimately to the nonprostanoids ONO-8713 (Fig. 5), with high selectivity for the $\mathrm{EP}_{1}$ receptor. ONO-8711 and ONO-8713 possess $K_{\mathrm{d}}$ values for mouse recombinant $\mathrm{EP}_{1}$ receptors of 1.7 and $0.3 \mathrm{nM}$, respectively (Watanabe et al., 1999, 2000; Naganawa et al., 2006). Small modifications may dramatically increase $\mathrm{EP}_{3}$ antagonist affinity. This series has also been widely employed to elucidate the therapeutic utility of $\mathrm{EP}_{1}$ antagonists.

Pharmacophores possessing potent $\mathrm{EP}_{1}$ antagonism with good CNS penetration have emerged over the last 10 years. The series reported by Merck (Ruel et al., 1999) contains a tricyclic system similar to the Searle series. A 
$E P_{1}$ antagonists<smiles>CC(C)CN(c1ccc(C(F)(F)F)cc1OCc1ccc(/C=C/C(=O)O)cc1)S(=O)(=O)c1ccco1</smiles><smiles>CC(C)(C)[13C](=O)[18O]</smiles>

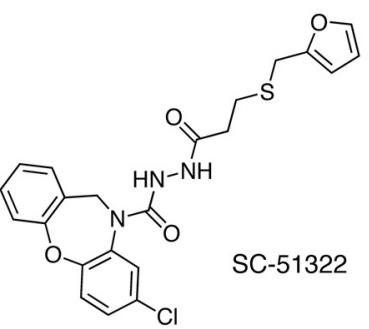<smiles>CC(C)Oc1ccc2c(=O)c3cc(C(=O)O)ccc3oc2c1</smiles>

$\mathrm{AH}-6809$

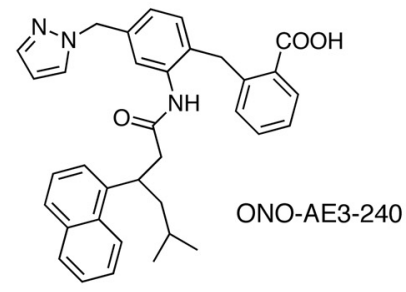

$\mathrm{EP}_{3}$ antagonists
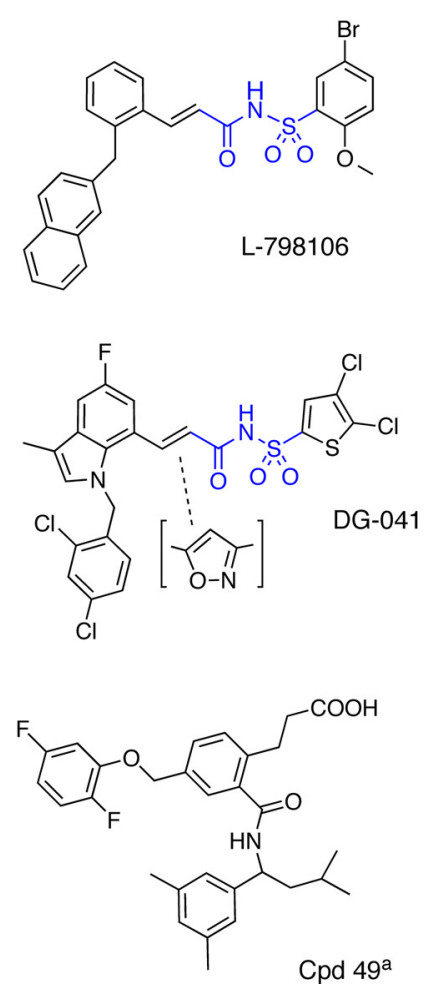

$E P_{4}$ antagonists
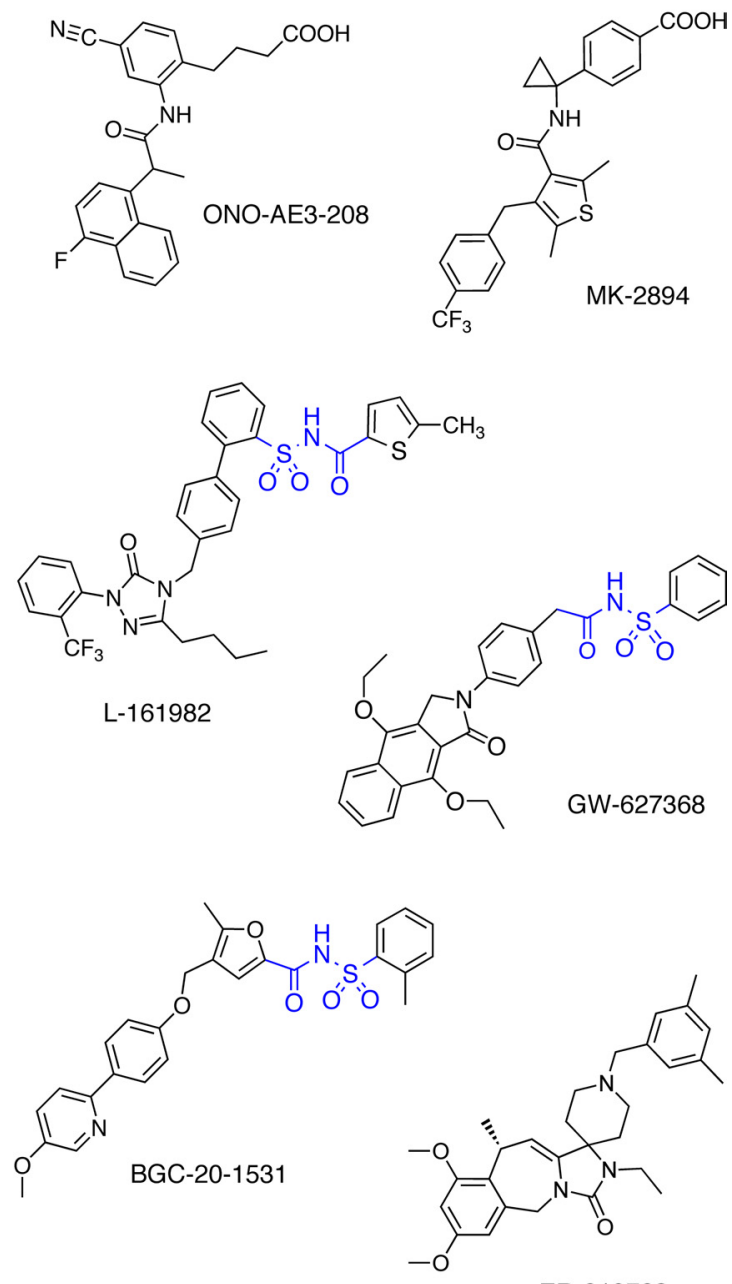

ER-819762

FIg. 5. Structures of representative $\mathrm{EP}_{1}, \mathrm{EP}_{3}$, and $\mathrm{EP}_{4}$ receptor antagonists. Heterocycle substitution in DG-041 maintains high $\mathrm{EP}_{3}$ affinity (Hategan et al., 2009). a, Asada et al. (2010). ONO-AE3-240, T. Maruyama, personal communication.

large number of 1,2-diaryl-thiophene/-cyclopentene analogs have been prepared as highly potent $\mathrm{EP}_{1}$ antagonists, including 1-(5-\{3-[2-(benzyloxy)-5-chlorophenyl]-2-thienyl\}pyridin3-yl)-2,2,2-trifluoroethane-1,1-diol (MF-266-1) (Ducharme et al., 2005; Clark et al., 2008) and GW-848687 (Giblin et al., 2007), shown in Fig. 5. Nonacidic analogs of GW-848687 (e.g., pyridylmethyl-amides) have been reported (Hall et al., 2007b,c).

e. Therapeutics. Very few potential medical uses for $\mathrm{EP}_{1}$ agonists have been presented to date. Moreover, the prospect of major unwanted side effects would be anticipated. Probably the most promising clinical application is for $\mathrm{EP}_{1}$ agonist control of impulsive behavior in psychiatric patients (Matsuoka et al., 2005). $\mathrm{EP}_{1}$ antagonists are quite a different matter and several therapeutic applications have been put forward. Indeed, $\mathrm{EP}_{1}$ receptors have been described as the downstream effectors of COX-2-induced neurotoxicity (Kawano et al., 2006). These proposed therapeutic applications are listed in Table 4, with only a brief reference to antino- ciceptive and anti-inflammatory activities, because these have been extensively reviewed (Jones et al., 2009). Despite considerable effort devoted to the design, synthesis, and testing of $\mathrm{EP}_{1}$ antagonists, no convincing evidence of clinical efficacy seems to have emerged. Clinical success seems limited to acid-induced visceral pain hypersensitivity (Sarkar et al., 2003), which arguably portends little for other indications.

2. EP $P_{2}$ Receptors.

a. Second messenger signaling. $\mathrm{EP}_{2}$ receptors are $\mathrm{G}_{\mathrm{s}}$-coupled and mediate increases in cAMP (Regan et al., 1994b). A positive feedback loop whereby cAMP signaling enhances $\mathrm{EP}_{2}$ receptor expression has been suggested (Sagana et al., 2009). Changes in cAMP levels produce pleiotropic effects by activating cAMP-binding proteins. These include PKAs, Epacs, and cAMP response element-binding protein regulation of gene transcription. The nature of the cellular responses to cAMP is also dependent on compartmentalization; in fact, the $\mathrm{EP}_{2}$ receptor seems to be excluded from caveolin-rich 
TABLE 4

Potential therapeutic application of $E P_{1}$ antagonists

\begin{tabular}{|c|c|c|c|c|c|c|}
\hline $\mathrm{EP}_{1}$ Antagonists & Route & Dose & Species & Experimental Model & Indication & Reference \\
\hline ONO-8711 & Intravenous & 1 and $3 \mathrm{mg} / \mathrm{kg}$ & Rat & Acetic acid-induced inflammation & Bladder inflammation & Ikeda et al., 2006 \\
\hline ONO-8711 & Dietary & 400 or $800 \mathrm{ppm}$ & Rat & PhIP-induced breast cancer & Breast cancer & Kawamori et al., 2001a,b \\
\hline ONO-8711 & Dietary & $250,500,1000 \mathrm{ppm}$ & Mouse & $\begin{array}{l}\text { Azoxymethane-induced aberrant } \\
\text { crypt foci, Min mice }\end{array}$ & Colon cancer & Watanabe et al., 1999 \\
\hline ONO-8713 & Oral & $20 \mathrm{mg} / \mathrm{kg}$ & Mouse & DNFB contact sensitivity & Contact dermatitis & Nagamachi et al., 2007 \\
\hline ONO-8713 & Topical & $50 \mu \mathrm{g}$ & Mouse & $\begin{array}{l}\text { UVB-induced inflammation and } \\
\text { tumor formation }\end{array}$ & Sunburn, skin cancer & Tober et al., 2006 \\
\hline ONO-8713 & Dietary & $250,5001000 \mathrm{ppm}$ & Mouse & $\begin{array}{l}\text { Azoxymethane-induced aberrant } \\
\text { crypt foci }\end{array}$ & Colon cancer & Watanabe et al., 2000 \\
\hline ONO-8713 & Intraventricular & $0-1-10 \mathrm{nM}$ & Mouse & NMDA excitotoxicity & Stroke & Ahmad et al., 2006a \\
\hline ONO-8713 & Intraperitoneal & $10 \mathrm{mg} / \mathrm{kg}$ & Mouse & Cerebral artery occlusion & Stroke & Ahmad et al., 2006a \\
\hline ONO-8713 & Intrathecal & 1 or $10 \mathrm{nmol}$ & Mouse & Neurochemical allodynia & Pain & Tsukamoto et al., 2010 \\
\hline PF-2907617-02 & Intravenous & $1 \mathrm{mg} / \mathrm{kg}$ & Rat & $\begin{array}{l}\text { Balloon catheter-induced } \\
\text { bladder outlet obstruction }\end{array}$ & $\begin{array}{l}\text { Overactive bladder } \\
\text { syndrome }\end{array}$ & Lee et al., 2007a,b \\
\hline SC-51089 & Intraperitoneal & $25 \mathrm{mg} / \mathrm{kg}$ & Mice & $\begin{array}{l}\text { KMG4 tumor xenografts on } \\
\text { SCID mice }\end{array}$ & Glioma & Matsuo et al., 2004 \\
\hline SC-51089 & Intraperitoneal & $10 \mathrm{mg} / \mathrm{kg}$ & Rat & $\begin{array}{l}\text { Lithium-pilocarpine status } \\
\text { epilepticus }\end{array}$ & Epilepsy & Pekcec et al., 2009 \\
\hline SC-51322 & Oral & $10 \mathrm{mg} / \mathrm{kg}$ & Rat & Spontaneously hypertensive rat & Hypertension & Guan et al., 2007 \\
\hline
\end{tabular}

PhIP, 2-amino-1-methyl-6-phenylimidazo[4,5-b]pyridine; SC-51089, dibenz(b,f)(1,4)oxazepine-10(11H)-carboxylic acid, 8-chloro-, 2-(1-oxo-3-(4-pyridinyl)propyl)hydrazide, monohydrochloride.

membrane fractions (Ostrom et al., 2001). The potential repertoire of $\mathrm{EP}_{2}$-mediated responses via cAMP-EpacsPKA-cAMP response element-binding protein has yet to be elucidated for many cells. The potential diversity may include ion channel function, $\left[\mathrm{Ca}^{2+}\right]$ signaling, ion transport, exocytosis, cell adhesion, and gap junction function formation (Holz et al., 2006). In addition, cAMP can influence several transcription factors by both PKA dependent and independent mechanisms (Sands and Palmer, 2008). Beyond $\mathrm{G}_{\mathrm{s}}$, it is probably better to describe cell signaling on a "cell-to-cell" basis. Finally, $\mathrm{EP}_{2}$ receptors inhibit the formyl-Met-Leu-Phe-induced phospholipase D pathway activation of neutrophils (Burelout et al., 2004) and cause Th1 cell differentiation (Yao et al., 2009), which are dependent on PI3K rather than cAMP.

b. Distribution and biological functions. $\mathrm{EP}_{2}$ receptors seem widely distributed, according to functional studies on isolated tissues, where they almost invariably produce relaxation (Coleman et al., 1994b). The earliest studies on mRNA expression suggested relatively low abundance and an uncertain distribution pattern (Regan et al., 1994b; Narumiya et al., 1999; Smock et al., 1999). In the past decade, a number of studies have indicated widespread distribution and important functions.

In the absence of potent and selective antagonists, gene deletion studies are of essential value in understanding the physiological and pathological roles of the $\mathrm{EP}_{2}$ receptor in living animals. In isolated tissues and cell culture, bioavailability is not an issue and therefore the $\mathrm{EP}_{2} / \mathrm{EP}_{1}$ antagonist AH-6809 (Fig. 5) may be used despite its low potency (Woodward et al., 1995b; Jones et al., 2009). In conjunction with selective agonists, the role of $\mathrm{EP}_{2}$ receptors is now better understood in many biological systems. $\mathrm{EP}_{2}$ receptors exert many inhibitory functions. In the general context of $\mathrm{PG}$ release and activity, the $\mathrm{EP}_{2}$ receptor could be considered to provide a major regulatory component in many instances.
$\mathrm{PGE}_{2}$ has long been known to be a bronchodilator with potential for treating asthma (Wasserman, 1981; Gardiner, 1986). The prospects improved with the discovery of selective $\mathrm{EP}_{2}$ agonists (Gardiner, 1986; Nials et al., 1993). $\mathrm{PGE}_{2}$-induced bronchodilation has been confirmed as $\mathrm{EP}_{2}$ receptor-mediated relaxation in isolated human bronchial preparations (Norel et al., 1999) and mouse airways (Sheller et al., 2000; Tilley et al., 2003; Hartney et al., 2006). $\mathrm{EP}_{4}$ receptors do not seem to mediate $\mathrm{PGE}_{2}$-induced relaxation of human bronchi (Norel et al., 1999). $\mathrm{EP}_{2}$ receptors also mediate substance P- and ATP-induced airway relaxation (Fortner et al., 2001). $\mathrm{EP}_{2}$ receptor activation on human airway smooth muscle cells may indirectly produce antiinflammatory effects. IL- $1 \beta$ releases granulocyte macrophage-colony-stimulating factor from human airway smooth muscle cells, which is inhibited by $\mathrm{EP}_{2}$ receptor stimulation (Clarke et al., 2004). Thus, the survival of infiltrating leukocytes by granulocyte macrophagecolony-stimulating factor would be abrogated by the action of $\mathrm{PGE}_{2}$ at $\mathrm{EP}_{2}$ receptors.

The tocolytic effect of $\mathrm{EP}_{2}$ agonists has also received attention. Suppression of spontaneous uterine activity has been reported with selective $\mathrm{EP}_{2}$ receptor agonists: $19(R)-\mathrm{OH} \mathrm{PGE}{ }_{2}$ in rabbit (Spilman et al., 1977; Woodward et al., 1993a) and butaprost in human preparations (Senior et al., 1991; Duckworth et al., 2002). $19(R)-\mathrm{OH} \mathrm{PGE}{ }_{2}$ increased uterine motility in a secondtrimester pregnant monkey (Spilman et al., 1977), but this effect did not transition into the human pregnant myometrium, where $\mathrm{EP}_{2}$ receptor stimulation produced tocolysis (Duckworth et al., 2002). Temporal and regional changes in $\mathrm{EP}_{2}$ receptor expression have also been implicated in pregnancy maintenance and laborassociated events. $\mathrm{EP}_{2}$ receptors decline toward term gestation (Brodt-Eppley and Myatt, 1999; Leonhardt et al., 2003), although they remain unaltered (BrodtEppley and Myatt, 1999; Astle et al., 2005: Sooranna et 
al., 2005) or increase during parturition (Grigsby et al., 2006). $\mathrm{EP}_{2}$ mRNA and nuclear $\mathrm{EP}$ receptors were most abundant in lower compared with upper segment tissues (Astle et al., 2005: Grigsby et al., 2006). This corresponds with a caudal decline in contractile responsiveness to $\mathrm{PGE}_{2}$, suggesting that $\mathrm{EP}_{2}$ receptors (Wikland et al., 1984) facilitate uterine distension for delivery of the fetus during labor. Rabbit oviductal motility was also suppressed by 19(R)-OH $\mathrm{PGE}_{2}$ (Spilman et al., 1977). Should this effect occur in humans, with a resultant slowdown in Fallopian tube movement, it could disrupt the timing for implantation. It could even result in an ectopic pregnancy, an unwanted event made more likely by $\mathrm{EP}_{2}$ receptor signaling as a contributory factor for fertilization and implantation ( $\mathrm{Lim}$ and Dey, 1997; Hizaki et al., 1999; Tamba et al., 2008).

$\mathrm{EP}_{2}$ receptors have long been known to relax vascular smooth muscle and produce a vasodepressor response (Armstrong et al., 1976; Audoly et al., 1999). The reninangiotensin system also regulates systemic blood pressure, and $\mathrm{EP}_{2}$ receptors are involved. In addition to controlling electrolyte and water homeostasis, the kidney also regulates blood pressure, and $\mathrm{EP}_{2}$ and $\mathrm{EP}_{4}$ receptors participate in $\mathrm{PGE}_{2}$-induced renin release (Schweda et al., 2004). At the juxtaglomerular cell level, $\mathrm{EP}_{2}$ and $\mathrm{EP}_{4}$ receptors were found to produce renin exocytosis (Friis et al., 2005). By measuring afferent arteriolar diameter, it was concluded that $\mathrm{EP}_{2}$ receptors partially mediate $\mathrm{PGE}_{2}$-induced vasodilatation (Imig et al., 2002). It has been claimed that $\mathrm{EP}_{2}$ receptors do not regulate overall renal hemodynamics, according to studies involving direct injection into mouse renal arteries and ultrasonic flowmetry to measure blood flow (Audoly et al., 2001). Intramedullary $\mathrm{PGE}_{2}$ infusion resulted in $\mathrm{EP}_{2}$-mediated renal $\mathrm{Na}^{+}$excretion (Chen et al., 2008). In mice lacking $\mathrm{EP}_{2}$ receptors, salt-sensitive hypertension occurs (Kennedy et al., 1999). These results suggest that $\mathrm{EP}_{2}$ receptors produce natriuresis and promote normotension in a high-salt dietary regimen. Protection of renal cystic epithelial cells from apoptosis has implicated the $\mathrm{EP}_{2}$ receptor in polycystic kidney disease (Elberg et al., 2007).

$\mathrm{PGE}_{2}$ has been proposed to influence a number of CNS functions by activating $\mathrm{EP}_{2}$ receptors. These range from activity-dependent synaptic plasticity against oxidative stress and acute excitotoxicity to a role in the development of COX-2-induced neurotoxicity. Evidence for $\mathrm{EP}_{2}$ and $\mathrm{EP}_{3}$ receptor involvement in long-term potentiation and long-term depression has been advanced; $\alpha$-amino-3 hydroxy-5-methyl-4-isoxazole-propionic acid receptor trafficking in the postsynaptic membrane was implicated as an underlying mechanism (Andreasson, 2010). The behavioral phenotype in $\mathrm{EP}_{2}$ receptor knockout mice was associated with a deficit in hippocampal longterm depression (Savonenko et al., 2009). $\mathrm{EP}_{2}$ receptors have also been implicated in $\mathrm{PGE}_{2}$ pain transmission (Ahmadi et al., 2002; Harvey et al., 2004; Reinold et al.,
2005), the mechanism involving blockade of inhibitory glycine receptors. Calcitonin-gene related peptide (CGRP) release from trigeminal neurons may be evoked by $\mathrm{EP}_{2}, \mathrm{DP}_{1}$, and IP receptors (Jenkins et al., 2001).

Because both $\mathrm{EP}_{2}$ and $\mathrm{EP}_{4}$ receptors are $\mathrm{G}_{\mathrm{s}}$-coupled G-protein-coupled receptors, it is not surprising that they share certain neurological functions. These include modification of tetrodotoxin-resistant $\mathrm{Na}^{+}$currents in neonatal rat nodose ganglia (Matsumoto et al., 2005) and protection against oxidative stress and amyloid $\beta$-peptide neurotoxicity (Echeverria et al., 2005). Nevertheless, there are several instances in which $\mathrm{EP}_{2}$ receptors are the singular cognate receptor mediating $\mathrm{PGE}_{2}$ mediated events.

$\mathrm{EP}_{2}$ receptor activation protects neurons against NMDA-receptor-mediated cytotoxicity, according to in vitro (Akaike et al., 1994; McCullough et al., 2004; Liu et al., 2005; Ahmad et al., 2006b) and living animal studies (Ahmad et al., 2006). $\mathrm{EP}_{2}$ receptors have actually been claimed to exacerbate NMDA-mediated cytotoxicity in the very same cells, cultured rat cortical neurons (Takadera and Ohyashiki, 2006), in which $\mathrm{EP}_{2}$-mediated neuroprotection was originally described (Akaike et al., 1994). In hippocampal neurons, caspase-dependent apoptosis seems to be produced by $\mathrm{PGE}_{2}$ acting through $\mathrm{EP}_{2}$ receptors (Takadera et al., 2004). Nevertheless, the majority of studies on neuronal cytotoxicity suggest that $\mathrm{EP}_{2}$ receptors are neuroprotective. In neuronal cells, $\mathrm{EP}_{2}$ signaling affords significant neuroprotection by a cAMP-PKA mechanism, whereas microglial $\mathrm{EP}_{2}$ receptor activation may lead to secondary neurotoxicity by up-regulating proinflammatory genes (Andreasson, 2010). Likewise, $\mathrm{EP}_{2}$ receptors may contribute to either neuroprotection or neurotoxicity by inducing brainderived neurotrophic factor release from microglial cells and astrocytes (Hutchinson et al., 2009). In astrocytes, it was reported that $\mathrm{EP}_{2}$ receptor stimulation elicits $\mathrm{Ca}^{2+}$ release from intracellular stores (Di Cesare et al., 2006).

A proinflammatory neurotoxic function for $\mathrm{EP}_{2}$ receptors has emerged during the past few years, notably with respect to innate immunity. LPS has been used to model innate immunity, because binding to CD14 and toll-like receptor 4 up-regulates pro-inflammatory genes, including COX-2 (Andreasson, 2010). $\mathrm{EP}_{2}$ receptors are highly inducible in the cerebral cortex and hippocampus (Zhang and Rivest, 1999). In hippocampal slice preparations, $\mathrm{EP}_{2}$ receptors exacerbate LPSinduced neurotoxicity (Wu et al., 2007). $\mathrm{EP}_{2}$, and $\mathrm{EP}_{1}$, receptors are very important in Toll-like receptor 4-induced depletion of intermediate progenitor cells destined for maturation in the hippocampal subgranular zone (Keene et al., 2009). In terms of hippocampal neurotransmission, postsynaptically synthesized $\mathrm{PGE}_{2}$ modulates transmission via presynaptic $\mathrm{EP}_{2}$ receptors (Sang et al., 2005). Activated microglial $\mathrm{EP}_{2}$ receptors seem to play a central role in the generation of reactive oxygen species and secondary neurotoxicity (Andreas- 
son, 2010). LPS-induced cerebral oxidative damage was selectively abolished in $\mathrm{EP}_{2}$ gene deletion studies (Montine et al., 2002). Additional, mostly deleterious, $\mathrm{EP}_{2}$ effects on microglia include $\alpha$-synuclein neurotoxicity (Jin et al., 2007), regulation of amyloid precursor protein (Pooler et al., 2004; Liang et al., 2005a), and inhibition of ATP-induced microglia migration (Nagano et al., 2008).

Neuroprotection has also attracted attention in vision research because sight is entirely dependent on optimal ocular and central neurotransmission. Experiments have been largely designed to assess vision-sparing potential in glaucoma (Woodward, 2000) and retinal disease (Andrade da Costa et al., 2009; Mori et al., 2009; Osborne et al., 2009). The underlying $\mathrm{EP}_{2}$-regulated signaling mechanisms involved in ocular neuroprotection have received little attention but are probably likely similar to those reported for CNS neurons. The link between sight-threatening ocular diseases and immune regulation is tenuous at best, and no attention has been paid to innate immunity.

Generally $\mathrm{EP}_{2}$ receptors tend to play anti-inflammatory roles, which is in contrast to their participations in innate immunity in the CNS. $\mathrm{EP}_{2}$ receptors inhibit Tcell proliferation according to MLR studies (Nataraj et al., 2001), regulate antigen-presenting cell function (Nataraj et al., 2001), inhibit TNF $\alpha$ release from bone marrow-derived dendritic cells (Vassiliou et al., 2003), inhibit major histocompatibility complex class II expression in dendritic cells (Harizi et al., 2003), suppress IFN $\alpha$ release by natural killer cells (Walker and Rotondo, 2004), are particularly effective in inhibiting Th1 and Th2 polarized antigen-specific T-cell responses (Okano et al., 2006), and augment the signaling and function associated with the anti-inflammatory cytokine IL-10 (Cheon et al., 2006). Several immune regulatory functions assigned to $\mathrm{EP}_{2}$ receptors are shared with $\mathrm{EP}_{4}$. These include Th1 differentiation (Yao et al., 2009), Th2 polarization (Kubo et al., 2004), and Th17 differentiation (Boniface et al., 2009; Napolitani et al., 2009; Narumiya, 2009; Yao et al., 2009). It should also be noted that numerous immunomodulatory effects of $\mathrm{PGE}_{2}$ are reported but not pharmacologically defined. Studies in living animals seem somewhat consistent with findings on immune competent cells: $\mathrm{EP}_{2}(-/-)$ mice treated with an $\mathrm{EP}_{4}$ antagonist exhibited a therapeutic effect in the collagen-induced arthritis model (Narumiya, 2009), which is consistent with in vitro T-cell studies.

In polymorphonuclear leukocytes and monocytes/ macrophages, $\mathrm{EP}_{2}$ receptors largely exert downregulatory functions. $\mathrm{EP}_{2}$ receptors inhibit neutrophil chemotaxis, superoxide generation, $\mathrm{LTB}_{4}$ release, and aggregation (Nials et al., 1993; Wheeldon and Vardey, 1993; Talpain et al., 1995; Yamane et al., 2000). LPSstimulated murine peritoneal neutrophils also secrete cytokines, and $\mathrm{EP}_{2}$ receptor activation augments IL-6 and granulocyte cell-stimulating factor release (Yamane et al., 2000; Sugimoto et al., 2005) but suppresses TNF $\alpha$ production to some extent (Yamane et al., 2000). Efferocytosis, ingestion of apoptotic cells by phagocytes, triggers the release of cytokines, $\mathrm{NO}$, and $\mathrm{PGE}_{2}$. Although this process may recover tissue homeostasis after injurious stimuli, it renders the lung, in particular, susceptible to secondary infection, because alveolar macrophages are impaired by apoptotic cells. $\mathrm{PGE}_{2}$, acting via $\mathrm{EP}_{2}$ receptors, mediates efferocytosis-induced inhibition of pulmonary macrophage antibacterial function (Medeiros et al., 2009). Such an effect may be mitigated by $\mathrm{EP}_{2}$-mediated monocyte/macrophage survival against free radical damage by peroxynitrite, a superoxide-nitric oxide coupling product that is an extremely reactive free radical (Tommasini et al., 2008). More pertinent to asthma and other type 1 allergies, $\mathrm{EP}_{2}$ receptors inhibit lung mast cell degranulation (Kay et al., 2006) and eosinophil trafficking (Sturm et al., 2008).

$\mathrm{PGE}_{2}$ has been implicated in osteoclast, chondrocyte, and synoviocyte function. $\mathrm{EP}_{2}$ and $\mathrm{EP}_{4}$ receptors often mediate similar effects, but responses to $\mathrm{EP}_{4}$ stimulation are typically more prominent. Small animal cell lines are used extensively, but results are contradictory (Graham et al., 2009). $\mathrm{EP}_{2}$ effects include osteoblast differentiation, osteoclast-induced bone resorption, and bone anabolic activity in living animal studies (Graham et al., 2009). It is also pertinent to note that $\mathrm{PGE}_{2}$ strongly inhibits human osteoclast formation (Take et al., 2005). In human and rat chondrocytes, $\mathrm{EP}_{2}$ receptors have quite opposite effects on proteoglycan accumulation: suppression (Li et al., 2009b) and enhancement (Miyamoto et al., 2003), respectively. $\mathrm{EP}_{2}$ receptors have been identified in synovial fibroblasts obtained from rheumatoid arthritis tissue specimens (Kojima et al., 2009).

Studies on $\mathrm{EP}_{2}$ receptor function in fibroblasts usually involves fetal or patient-derived lung fibroblasts. $\mathrm{EP}_{2}$ receptors have been shown to produce diverse effects that may limit fibrosis and scar formation. It is noteworthy that $\mathrm{EP}_{2}$ receptors inhibit the transition of human lung fibroblasts to myofibroblasts, which are the hallmark of pulmonary fibrotic disease (Kolodsick et al., 2003). Furthermore, $\mathrm{PGE}_{2}$ inhibits fibroblast proliferation and collagen expression in patient-derived lung fibroblasts (Huang et al., 2007b) and migration (White et al., 2005) via $\mathrm{EP}_{2}$ receptor activation. It is pertinent that bleomycin, which produces fibrogenesis, produces a loss of $\mathrm{EP}_{2}$ expression in pulmonary fibroblasts with a resultant loss of the inhibitory effect of $\mathrm{PGE}_{2}$ on collagen biosynthesis and proliferation (Moore et al., 2005). Finally, it is of interest that $\mathrm{EP}_{2}$ receptors protect human lung fibroblasts from cigarette smoke-induced apoptosis (Sugiura et al., 2007). Tissue destruction associated with periodontitis and healing may also involve $\mathrm{EP}_{2}$ receptors expressed by fibroblasts (Noguchi et al., 2002; Weinberg et al., 2009). In view of the importance of fibroblasts in cutaneous wound healing and scar formation, dermal fibroblasts have also received attention. Maintenance of 
the migratory phenotype may be important for remodeling and regenerative repair, an effect achievable by $\mathrm{EP}_{2}$ receptor activation (Parekh et al., 2007). Reduced $\mathrm{EP}_{2}$ expression causes increased collagen synthesis in keloid fibroblasts (Hayashi et al., 2006).

The role of $\mathrm{EP}_{2}$ receptors in skin tumor development has been the subject of several investigations. $\mathrm{EP}_{2}$ receptors are claimed to induce COX-2 (Ansari et al., 2007) and to participate in tumor formation (Sung et al., 2006; Chun et al., 2009). Loss of $\mathrm{EP}_{2}$ receptors from keratinocytes has been suggested as a mechanism for neoplastic progression resulting from a more invasive phenotype, although $\mathrm{EP}_{2}$ receptors protect against UV-induced carcinogenesis (Konger et al., 2002; Brouxhon et al., 2007). The immune regulatory effects associated with $\mathrm{EP}_{2}$ receptors, which may be beneficial in relieving cutaneous hypersensitivity, can result in diminished antitumor immune responses by virtue of reduced cytotoxic T-cell responses and inhibition of dendritic cell differentiation (Yang et al., 2003).

It seems to be generally accepted that COX inhibitors are of value in treating colorectal cancer (Clevers, 2006). A significant role for $\mathrm{PGE}_{2}$ in producing a signaling cascade involving phosphorylation of glycogen synthase kinase $3, \beta$-catenin translocation to the nucleus, and resultant Tcf/Lef transcription and COX-2 up-regulation in the development of chronic inflammation and colon cancer has been elucidated (Fujino et al., 2002; Regan, 2003) and subsequently confirmed (Castellone et al., 2005). Because $\mathrm{EP}_{2}$ receptors are claimed to have a central role in colon cancer cells (Sonoshita et al., 2001; Seno et al., 2002), the role of $\mathrm{EP}_{2}$ receptors in other carcinomas has been the subject of intense activity.

The $\mathrm{EP}_{2}$-dependent angiogenesis associated with mouse intestinal tumors (Seno et al., 2002) has been further studied in pulmonary endothelial cells, aortic rings, and the cornea (Kamiyama et al., 2006). It was concluded that $\mathrm{EP}_{2}$ receptors are a major factor in endothelial cell motility (Kamiyama et al., 2006). $\mathrm{EP}_{2}$ receptors, in addition to $\mathrm{EP}_{4}$ receptors, produce angiogenesis in prostate cancer (Jain et al., 2008). $\mathrm{EP}_{2}$ and $\mathrm{EP}_{4}$ have also been implicated in VEGF expression and increased invasiveness of ovarian carcinoma cells by stimulating tumor-associated matrix metalloproteases (Spinella et al., 2004). $\mathrm{EP}_{2}$ receptors may play a role in breast cancer by inducing aromatase (Brueggemeier et al., 2001; Subbaramaiah et al., 2008) and VEGF induction and hyperplasia in mammary tumor cells (Chang et al., 2005a,b). $\mathrm{EP}_{2}$ receptor deficiency decreased the growth, angiogenesis, and metastasis of mammary tumors in mice; increased $\mathrm{EP}_{2}$ receptor expression by $\mathrm{TGF} \beta$ increased mammary epithelial cell invasion, growth, and resistance to TGF $\beta$-mediated cytostasis (Tian and Schiemann, 2010). Proliferation of squamous cell carcinoma has been ascribed to $\mathrm{EP}_{2}$ receptors (Donnini et al., 2007; Yu et al., 2008a, 2009). PPAR $\gamma$ ligands were shown to inhibit lung carcinoma cell proliferation by suppressing $\mathrm{EP}_{2}$ receptor expression (Han and Roman, 2004). In complete contrast, $\mathrm{EP}_{2}$ and $\mathrm{EP}_{4}$ receptors inhibit human gastric carcinoma cell lines (Okuyama et al., 2002).

c. Gene deletion studies. No less than three different lines of $\mathrm{EP}_{2}(-/-)$ mice have been generated (Hizaki et al., 1999; Kennedy et al., 1999; Tilley et al., 1999). $\mathrm{EP}_{2}$ receptor-deficient mice exhibited reduced fertility; they became pregnant and delivered at term, but the litter sizes were reduced (Hizaki et al., 1999; Kennedy et al., 1999; Tilley et al., 1999). This seems to be due to failure of the released ovum to be fertilized by virtue of incomplete expansion of the cumulus (Hizaki et al., 1999; Tilley et al., 1999); cumulus cells are needed for oocyte maturation-associated reduction in $\mathrm{TNF} \alpha$-stimulated gene 6 (TSG-6) expression, which seems to be the $\mathrm{EP}_{2}$ receptor-mediated effect (Ochsner et al., 2003).

$\mathrm{EP}_{2}$ receptor deletion also affects blood pressure. Resting blood pressure for mice on a normal diet is reduced but may be restored by providing excess salt in the diet (Tilley et al., 1999). Neither plasma renin activity nor renin mRNA were elevated in $\mathrm{EP}_{2}(-/-)$ animals (Tilley et al., 1999). The vasodilator responses to $\mathrm{PGE}_{2}$, per se, seems to be $\mathrm{EP}_{2}$ receptor-mediated, and $\mathrm{PGE}_{2}$ produces considerable hypertension in $\mathrm{EP}_{2}(-/-)$ mice (Kennedy et al., 1999). In one study, a high-salt diet resulted in a rapid and sustained increase in blood pressure (Kennedy et al., 1999). The degree of salt-sensitive hypertension recorded in the two studies was different and, in contrast to Tilley et al. (1999), Kennedy et al. (1999) reported an elevation in resting blood pressure. These findings are not readily explained; they may reflect different genetic backgrounds of the mice used in each study. There is also incongruence with respect to renal hemodynamics. Deletion of $\mathrm{EP}_{2}$ receptors had little effect on $\mathrm{PGE}_{2}$-induced renal vasodilation and did not alter resting renal blood flow (Audoly et al., 2001). Quite different and unexpected results were obtained by monitoring afferent renal arteriolar caliber. The vasodilator response to $\mathrm{PGE}_{2}$, as measured by arteriolar diameter, in wild-type mice was not merely abolished by $\mathrm{EP}_{2}$ receptor deletion but was converted to a vasoconstrictor response (Imig et al., 2002). The precise reason for this apparent discrepancy is not clear. It may reflect different technologies: ultrasonic flowmeter (Audoly et al., 2001) versus measurement of arteriolar diameter by transillumination videomicroscopy (Imig et al., 2002). $\mathrm{EP}_{2}$ receptors have been shown also to be involved in natriuresis, according to gene deletion studies. Thus, a high-salt diet increases $\mathrm{PGE}_{2}$ biosynthesis in the renal medulla, which promotes $\mathrm{EP}_{2}$ receptor-mediated sodium excretion (Chen et al., 2008).

The CNS has been a major focus of $\mathrm{EP}_{2}$ receptor deficiency studies, where the neuroprotective properties of $\mathrm{EP}_{2}$ receptors have been confirmed and extended. Thus, gene deletion resulted in a greater infarct volume in the cerebral artery occlusion-reperfusion model of 
transient forebrain ischemia (McCullough et al., 2004). $\mathrm{EP}_{2}$ receptor deletion reduced oxidative damage and amyloid burden in a model of Alzheimer's disease, however (Liang et al., 2005a). In $\mathrm{EP}_{2}$-deficient mice, there is altered long-term synaptic plasticity in the hippocampus and impaired spatial learning (Yang et al., 2009). In a separate study using the same test (i.e., the Morris water maze to test hippocampal dependent spatial memory), $\mathrm{EP}_{2}(-/-)$ mice demonstrated no deficits in spatial memory (Savonenko et al., 2009). In studies of $\mathrm{EP}_{2}$ receptor involvement behavior and cognition, there were cognitive deficits in tests for fear and social memory (Savonenko et al., 2009). $\mathrm{EP}_{2}(-/-$ ) mice also exhibited impaired prepulse inhibition and heightened anxiety. The complex behavioral phenotype observed was attributed to a long-term depression deficit in the hippocampus (Savonenko et al., 2009). It is noteworthy that mice lacking $\mathrm{EP}_{2}$ receptors completely lacked spinal hyperalgesia in response to $\mathrm{PGE}_{2}$ (Reinold et al., 2005). This was identified by electrophysiology as diminished synaptic inhibition of excitatory dorsal horn neurons.

Neuronal cytotoxicity is intimately associated with innate immunity. LPS, which stimulates innate immunity by binding to CD14 and activating toll-like receptor 4, produces cerebral oxidative damage that was almost abolished in $\mathrm{EP}_{2}$ receptor-deficient mice (Montine et al., 2002). Innate immunity-mediated neurodegeneration produced by LPS was subsequently determined to be critical to $\mathrm{EP}_{2}$ receptors expressed by microglia (Shie et al., 2005). COX-2 and inducible nitric-oxide synthase are also implicated in LPS-induced neurodegeneration. The roles of $\mathrm{EP}_{2}$ receptors in acquired immunity are arguably worthy of further study, notably because excessive concentrations of $\mathrm{PGE}_{2}$ have been used in some key human cell studies. An important role for the $\mathrm{EP}_{2}$ receptor in inhibition of dendritic cell differentiation and function has been claimed (Yang et al., 2003). In the MC26 tumor model, there was improved dendritic cell function and number in tumor-bearing $\mathrm{EP}_{2}(-/-)$ mice (Yang et al., 2003). This reflects $\mathrm{EP}_{2}$ receptor-mediated tumor immunosuppression and inhibition of the host reaction to tumor progression. In a different setting, 7,12-dimethylbenz( $a$ )anthracene/12- $O$-tetradecanoylphorbol-13-acetate-induced skin tumors, $\mathrm{EP}_{2}(-/-)$ mice showed suppressed skin tumor development associated with decreased proliferation, angiogenesis, inflammation, and cell survival (Sung et al., 2005). The link between COX-2 and $\mathrm{EP}_{2}$ receptors is also manifest in a study on mammary hyperplasia, which was reduced in $\mathrm{EP}_{2}(-/-)$ mice (Chang et al., 2005a).

Bone deposition and resorption has been a popular avenue of study using $\mathrm{EP}_{2}$-deficient mice. In cocultures of spleen and calvarial osteoblasts, the response to $\mathrm{PGE}_{2}$, or even PTH, was greatly reduced when both of these cell types were obtained from $\mathrm{EP}_{2}(-/-)$ mice; the data in total suggest that $\mathrm{EP}_{2}$ receptors activate osteoblastic cells to stimulate osteoclast formation ( $\mathrm{Li}$ et al.,
2000). Osteoclast formation from cells of an osteoblastic lineage requires up-regulation of RANKL and macrophage colony-stimulating factor. $\mathrm{PGE}_{2}$-induced increases in RANKL expression were reduced in cells derived from both $\mathrm{EP}_{2}(-/-)$ and $\mathrm{EP}_{4}(-/-)$ mice (Li et al., 2002); this was the major effect observed. A biphasic effect of $\mathrm{PGE}_{2}$ on osteoclast formation was apparent, the secondary stimulatory effect being $\mathrm{EP}_{2}$ receptormediated (Ono et al., 2005). Finally, in $\mathrm{EP}_{2}(-/-)$ mice, bone exhibited weak biomechanical properties compared with that obtained from wild-type control mice (Akhter et al., 2001).

d. Agonists and antagonists. Misoprostol (Collins et al., 1985), in which the 15-hydroxyl group of $\mathrm{PGE}_{1}$ is displaced to C16, shows modest selectivity for $\mathrm{EP}_{2}$ and $\mathrm{EP}_{3}$ receptors, whereas the related butaprost (TR4979) is $\mathrm{EP}_{2}$-selective (Gardiner, 1986). However, tissue-dependent hydrolysis of its $\mathrm{C} 1$ ester is required to obtain full bioactivity ( $K_{\mathrm{i}}$ : butaprost, $3500 \mathrm{nM}$; butaprost-free acid, $91 \mathrm{nM}$ for human $\mathrm{rc}-\mathrm{EP}_{2}$ receptor; Abramovitz et al., 2000). There has been some confusion over the configuration at $\mathrm{C} 16$ of butaprost used in the original reports (see http://www.caymanchem.com/app/template/ Product.vm/catalog/10006045 for resolution of this issue). Commercially available butaprost-FA (Fig. 4) is the more active (racemic) $16 S$ epimer. The "2-series" analog of butaprost-FA (CAY-10399) also shows high $\mathrm{EP}_{2} / \mathrm{EP}_{4}$ selectivity and is devoid of the IP agonism present in butaprost-FA (Tani et al., 2002a,b ). Retaining the $16 S$ configuration and replacing the 9-ketone with a $\beta$-chloro group generated highly potent and selective $\mathrm{EP}_{2}$ agonists (Tani et al., 2002a,b). One of these, ONO-AE1-259 (Fig. 4), has proved valuable in characterizing inhibitory $\mathrm{EP}_{2}$ systems (Cao et al., 2002; Clarke et al., 2004) and has proved useful when both $\mathrm{EP}_{4}$ and IP receptor systems are also present (Jones and Chan, 2005).

Acyclic derivatives of $\mathrm{PGE}_{1}$ with a $N^{8}$-methylsulfone structure exhibited weak, but selective, $\mathrm{EP}_{2}$ agonism (Jones et al., 1977). CP-533536 (Fig. 4) is a related nonprostanoid with subnanomolar $\mathrm{EC}_{50}$ for the rat rc$\mathrm{EP}_{2}$ receptor (Li et al., 2003; Cameron et al., 2009). $\mathrm{EP}_{2}$ full agonism was also found for another nonprostanoid (Fig. 4, compound 9; from Belley et al., 2005) during screening for $\mathrm{EP}_{3}$ antagonism.

There are no potent and selective $\mathrm{EP}_{2}$ receptor antagonists available. AH-6809 (Fig. 5) remains the most useful compound in this class (Woodward et al., 1995b), despite significant activity at $\mathrm{EP}_{1}$ receptors (Coleman et al., 1994b). Presumably, there has been inadequate rationale and commercial incentive to design potent and selective $\mathrm{EP}_{2}$ antagonists. Potential uses, such as for innate immunity and secondary pulmonary infection, would likely be effectively treated by COX-2 inhibitors.

e. Therapeutics. The therapeutic potential of $\mathrm{EP}_{2}$ agonists has been under consideration for more than 2 decades; so far, however, no clinically useful drugs have 
emerged. Indications for $\mathrm{EP}_{2}$ agonist therapy involving relief of smooth muscle spasm have been known for at least 2 decades. These include tocolysis, for the prevention of preterm labor and dysmenorrhoea (Senior et al., 1993; Duckworth et at., 2002) and bronchodilatation for asthma treatment (Gardiner, 1986; Nials et al., 1993), which would be analogous to $\beta_{2}$-adrenoceptor therapy for relieving bronchospasm. An additional dimension to $\mathrm{EP}_{2}$ agonist therapy is the inhibitory effect on eosinophil infiltration (Sturm et al., 2008) and lung mast cell degranulation (Kay et al., 2006). AH 13205 was not a clinical success and caused airway irritation in human volunteers (Nials et al., 1993). Although highly selective, $\mathrm{AH} 13205$ is not a potent $\mathrm{EP}_{2}$ agonist (Regan et al., 1994b). Perhaps asthma therapy should be revisited using the more potent compounds that have been discovered during the past few years.

Bone anabolic therapy with both $\mathrm{EP}_{2}$ and other prostanoid agonists has been studied for several years (Hartke and Lundy, 2001; Graham et al., 2009). $\mathrm{EP}_{2}$ agonists, even within the prostanoid class of drugs, are not the only options for treating osteoporosis and enhancement of fracture healing. Likewise, several prostanoid receptors mediate ocular hypotension. $\mathrm{EP}_{2}$ agonists are, however, particularly efficacious at lowering intraocular pressure, and even butaprost can restore laserinduced ocular hypertension to an ocular normotensive state (Nilsson et al., 2006). A novel series of $\mathrm{EP}_{2}$ agonists of a nonprostanoid structure have been reported to be extraordinarily potent, efficacious, and long-acting (Coleman and Middlemiss, 2009). The potential therapeutic utility of $\mathrm{EP}_{2}$ agonists is summarized in Table 5.

3. $E P_{3}$ Receptors.

a. Second messenger signaling. Pharmacological characterization of $\mathrm{EP}_{3}$ receptors revealed smooth muscle contractibility (Coleman et al., 1994b) and pertussis toxin sensitivity (Sonnenburg et al., 1990), which predicted a more promiscuous $\mathrm{G}$ protein-coupling repertoire. This has been proven to be the case to some extent. The major signaling pathway for $\mathrm{EP}_{3}$ receptors is $\mathrm{G}_{\mathrm{i}^{-}}$ induced adenylate cyclase inhibition (Narumiya et al., 1999). Numerous alternatively spliced $\mathrm{EP}_{3}$ variants have been identified (Sugimoto et al., 1993; Breyer et al., 1994; Regan et al., 1994a; Takeuchi et al., 1994; Kotani et al., 1995; Schmid et al., 1995; Kotelevets et al., 2007). $\mathrm{EP}_{3}$ mRNA splicing variants are reported to subserve diverse receptor functions. The $\mathrm{EP}_{3}$ receptor carboxyl terminus is essential for $\mathrm{G}$ protein activation (Irie et al., 1994) and alternative splicing variants thereof determine agonist and constitutive $\mathrm{G}_{\mathrm{i}}$ activity (Hasegawa et al., 1996; Negishi et al., 1996; Hizaki et al., 1997; Jin et al., 1997). Moreover, these alternative splicing variants confer a wide repertoire of signaling pathways. In addition to decreasing cAMP levels, PI turnover, and increased intracellular $\mathrm{Ca}^{2+}$ have been reported for $\mathrm{EP}_{3}$ isoforms (Namba et al., 1993; An et al., 1994; Takeuchi et al., 1994; Schmid et al., 1995; Yamaoka et al., 2009). $\mathrm{EP}_{3}$ isoforms also activate Rho via pertussis toxin-sensitive G protein(s) (Katoh et al., 1996; Hasegawa et al., 1997), via $\mathrm{G}_{12}$ and possibly $\mathrm{G}_{13}$ (Hasegawa et al., 1997; Macias-Perez et al., 2008). $\mathrm{EP}_{3}$ receptor stimulation produces neurite retraction via small GTPase Rho (Katoh et al., 1996). A role for Rho kinase in $\mathrm{EP}_{3}$ receptor-induced smooth muscle contraction was indicated by agreement between the potencies of Rho kinase inhibitors for suppression of sulprostone-induced contraction in guinea pig aorta and their reported potencies on the isolated enzyme system (Shum et al., 2003). However, in the case of "silent" $\mathrm{EP}_{3}$ contraction/synergism in the rat femoral artery preparation, Rho kinase inhibition suppressed both the priming agent response and the enhanced response to sulprostone, implicating Rho kinase as a common late-stage transduction process (Hung et al., 2006). Synergism between agonists on smooth-muscle $\mathrm{EP}_{3}$ agonist-dependent $\mathrm{G}_{\mathrm{s}}$ activity also occurs (Negishi et al., 1996), which seems to depend on interaction between the arginine residue in the seventh transmembranespanning segment and the carboxylate anion of the ligand (Negishi et al., 1995), as reported for the $\mathrm{EP}_{3} \mathrm{D}$ isoform. The differential function of $\mathrm{EP}_{3} \mathrm{mRNA}$ splicing variants even extends to receptor internalization (Bilson et al., 2004) and membrane targeting (Hasegawa et al., 2000). No consistent nomenclature for alternative splicing variants of $\mathrm{EP}_{3}$ or other prostanoid receptors has been agreed upon.

b. Distribution and biological functions. In situ hybridization, Northern blotting, and functional studies have revealed a wide distribution for $\mathrm{EP}_{3}$ receptors.

TABLE 5

Potential therapeutic application of $\mathrm{EP}_{2}$ agonists

\begin{tabular}{|c|c|c|c|c|c|c|}
\hline Agonist & Route & Dose & Species & Experimental Model & Indication & Reference \\
\hline AH 13205 & Aerosol & $0.1-3 \mathrm{nM}$ & $\begin{array}{l}\text { Guinea } \\
\text { pigs }\end{array}$ & $\begin{array}{l}\text { Histamine induced } \\
\text { bronchoconstriction }\end{array}$ & Asthma & Nials et al., 1993 \\
\hline Butaprost & $\begin{array}{l}\text { Tissue culture vs. } \\
\text { oxytocin }\end{array}$ & $10^{-6} \mathrm{M}$ infusion & Human & $\begin{array}{l}\text { Isolated human uterine } \\
\text { specimens from pregnant } \\
\text { and nonpregnant donors }\end{array}$ & $\begin{array}{l}\text { Prevention of preterm } \\
\text { labor; } \\
\text { dysmenorrhoea }\end{array}$ & Duckworth et al., 2002 \\
\hline Butaprost & Topical (ocular) & $0.1 \%$ & Monkey & $\begin{array}{l}\text { Laser-induced glaucomatous } \\
\text { monkey }\end{array}$ & Glaucoma & Nilsson et al., 2006 \\
\hline $\begin{array}{l}\mathrm{CP} \\
533536\end{array}$ & Local implant & $10,50,100 \mathrm{mg} / \mathrm{ml}$ & Dog & Canine ulnar critical defect & Fracture healing & Paralkar et al., 2003 \\
\hline $\begin{array}{l}\mathrm{CP} \\
\quad 533536\end{array}$ & Topical (ocular) & $0.01-0.1 \%$ & $\begin{array}{l}\text { Dog, } \\
\text { monkey }\end{array}$ & $\begin{array}{l}\text { Laser-induced glaucomatous } \\
\text { monkey }\end{array}$ & Glaucoma & Woodward and Chen, 2007 \\
\hline
\end{tabular}


They seem to play important roles in the CNS, cardiovascular system, reproductive system, kidney, and urinary bladder. In common with most other prostanoid receptors, $\mathrm{EP}_{3}$ receptors have been implicated in cancer and inflammation/immune regulation.

$\mathrm{EP}_{3}$ receptors are widely expressed in the CNS and specifically localized to neurons (Sugimoto et al., 1994b). $\mathrm{EP}_{3}$ mRNA was most abundant in neurons in the sensory ganglia (Sugimoto et al., 1994b). This would be consistent with studies on acute herpetic pain, where $\mathrm{PGE}_{2}$ content is increased in the dorsal root ganglia and analgesia was produced by an $\mathrm{EP}_{3}$ antagonist and in $\mathrm{EP}_{3}(-/-)$ mice (Takasaki et al., 2005). Gene deletion studies have also revealed a role for $\mathrm{EP}_{3}$ receptors in $\mathrm{PGE}_{2}$-induced hyperalgesia (Minami et al., 2001), LPS enhanced acetic acid-induced writhing (Ueno et al., 2001), and allodynia produced by intrathecal administration of HIV-1 glycoprotein gp120 (Minami et al., 2003). A mechanistic study on sensory ganglia demonstrated that $\mathrm{EP}_{3}$ receptors attenuate desensitization of $\mathrm{B} 2$ receptors, thereby restoring the response to bradykinin (Kozaki et al., 2007). $\mathrm{EP}_{3}$ receptors located in higher centers have been implicated in mediating hyperalgesia (Oka et al., 1994; Hosoi et al., 1997; Oliva et al., 2006).

Aspirin has been used to reduce fever for over a century, now a detailed insight into the pharmacology is available. $\mathrm{EP}_{3}$ receptors play a major role in hyperpyrexia. An impaired febrile response in $\mathrm{EP}_{3}(-/-)$ mice was reported (Ushikubi et al., 1998). $\mathrm{EP}_{3}$ receptors in the median preoptic nucleus have been demonstrated as critical for sickness-induced fever (Lazarus et al., 2007; Furuyashiki and Narumiya, 2009). Studies on EP receptor-specific ligands also implicate $\mathrm{EP}_{3}$ receptors in producing fever but, somewhat surprisingly, also implicate $\mathrm{EP}_{1}$ receptors (Oka et al., 2003b). Subsequent studies have focused on $\mathrm{EP}_{3}$ receptors. $\mathrm{EP}_{3}$ receptors have been proposed to mediate brown adipose tissue thermogenesis, although supportive pharmacological evidence is lacking (Yoshida et al., 2003). The preoptic area expresses $\mathrm{EP}_{3}$ receptors that provide direct pyrogenic input to two hyperpyrexia generating sympathoexcitatory brain regions, the dorsomedial hypothalamus (Nakamura et al., 2005), and the rostral raphe pallidus nucleus (Nakamura et al., 2009). It has been suggested that $\mathrm{EP}_{3}$ receptors cause a decrease in preoptic $\mathrm{GABA}_{\mathrm{A}}$ expression as a mechanism for $\mathrm{PGE}_{2}$-induced fever (Tsuchiya et al., 2008).

The abundance (Sugimoto et al., 1992) and widespread distribution of $\mathrm{EP}_{3}$ receptors in the brain (Sugimoto et al., 1994b) point to multiple CNS functions and even opportunities for novel therapies. This opportunity does not seem to have been pursued to its fullest extent. Nevertheless, some progress has been made. $\mathrm{EP}_{3}$ receptor mRNA was found to be associated with monoaminergic neurons in the brainstem, and $\mathrm{EP}_{3}$ receptors were postulated as performing a modulatory function (Naru- miya et al., 1999). In mouse cerebral cortex slices, $\mathrm{EP}_{3}$ and histamine $\mathrm{H}_{3}$ receptors located presynaptically inhibited norepinephrine in a partially exclusive manner (Schlicker and Marr, 1997), but beyond this $\mathrm{EP}_{3}$, modulation of monoamine neurotransmitter release does not seem to have been diligently pursued. One study points to $\mathrm{PGE}_{2}$-activated sympathetic nerve activity in the brain stem, with resultant tachycardia and hypertension (Ariumi et al., 2002). Behavioral suppression produced by IL- $1 \beta$ on naloxone-induced withdrawal jumping in morphine-dependent mice (Nakagawa et al., 1995) and $\Delta^{8}$-tetrahydrocannabinol on lever-pressing behavior (Yamaguchi et al., 2004) both seem to involve $\mathrm{PGE}_{2}$ acting through $\mathrm{EP}_{3}$ receptors. Finally, reduced brain injury has been reported after cerebral ischemia in mice lacking $\mathrm{EP}_{3}$ receptors (Saleem et al., 2009b) and ischemic excitotoxicity was reduced by the $\mathrm{EP}_{3}$ antagonist ONO-AE3-240 (Fig. 5) (Ikeda-Matsuo et al., 2010).

The major foci of cardiovascular $\mathrm{EP}_{3}$ receptor research have been myocardial injury and platelets. The cardiovascular system, however, may provide a large-scale operational model of $\mathrm{EP}_{3}$ receptor influence on monoaminergic neuronal function in the CNS. This would be provided by the pithed rat model, where presynaptic $\mathrm{EP}_{3}$ receptors inhibit the release of catecholamines and the resultant vasopressor response (Malinowska et al., 1994). Platelet aggregation studies have largely concentrated on $\mathrm{TP}$ and IP receptors, but $\mathrm{EP}_{3}$ receptors seem to have a more subtle role in platelet aggregation. $\mathrm{PGE}_{2}$ exerts a dual action on platelets, inhibition at high doses, and potentiation of the effect of proaggregatory agents (Armstrong, 1996). This potentiating effect was ascribed to $\mathrm{EP}_{3}$ receptors (Matthews and Jones, 1993), which has been amply confirmed (Fabre et al., 2001; Ma et al., 2001; Gross et al., 2007; Singh et al., 2009). $\mathrm{EP}_{3}$ agonists have been shown to reduce infarct size and reduce myocardial injury (Zacharowski et al., 1999; Hohlfeld et al., 2000), with supportive evidence from cardiospecific $\mathrm{EP}_{3}$ receptor overexpression (Martin et al., 2005). In the kidney, an $\mathrm{EP}_{3}$ vasoconstrictor effect has been observed for the intralobular arteries (van Rodijnen et al., 2007).

In 2008, a series of articles implicated $\mathrm{EP}_{3}$ receptors in bladder micturition. First, a report on increased bladder capacity in $\mathrm{EP}_{3}(-/-)$ mice was reported (McCafferty et al., 2008). Infusion of the $\mathrm{EP}_{3}$ agonist GR 63799X into the bladder of wild-type mice reduced bladder capacity, implicating $\mathrm{EP}_{3}$ receptors as a contributing factor to overactive bladder (McCafferty et al., 2008). At the same time, two studies on peripheral and central neuronal control of bladder function were published. The $\mathrm{EP}_{3}$ antagonist DG-041 (Fig. 5) selectively inhibited responses of mechanosensitive afferent nerves to urinary bladder distension, inhibited the visceromotor response to bladder distension, and reduced the frequency of rhythmic bladder motility (Su et al., 2008a). A second study demonstrated that intrathecal or intracerebroven- 
tricular administration of potent $\mathrm{EP}_{3}$ antagonists reduced the frequency of bladder contractions, albeit not their amplitude (Su et al., 2008b). The visceromotor reflex response was more effectively inhibited by intrathecal dosing, suggesting that bladder nociception primarily involves spinal $\mathrm{EP}_{3}$ receptors ( $\mathrm{Su}$ et al., 2008b). The potential utility of $\mathrm{EP}_{3}$ antagonists for treating detrusor hyperactivity and pain associated with bladder disorders may be offset by a study showing that $\mathrm{EP}_{3}$ receptors cause hypercontractility in obstructed urethra (Ankem et al., 2005).

$\mathrm{EP}_{3}$ receptor mRNA has been found in parietal and chief cells of the gastric fundic epithelium (Narumiya et al., 1999). This is consistent with $\mathrm{EP}_{3}$-mediated inhibition of gastric acid secretion (Bunce et al., 1991; Perkins et al., 1991; Savage et al., 1993; Yokotani et al., 1996; Kato et al., 2005; Dey et al., 2006) in rats. This $\mathrm{EP}_{3}$ inhibitory mechanism seems widely held to be true for humans, although actual functional evidence cannot be found. $\mathrm{EP}_{3}$ receptors, however, have been shown to be expressed throughout the human gastric epithelium (Takafuji et al., 2002). It was proposed that $\mathrm{EP}_{3}$ receptors may control gastrointestinal smooth muscle contraction because expression was found in longitudinal smooth muscle and in neurons of the myenteric ganglia (Narumiya et al., 1999). $\mathrm{EP}_{3}$ receptors increase slowwave peristaltic frequency in mice (Forrest et al., 2009). The presence of $\mathrm{EP}_{3}$ receptors in the duodenum seems essential for $\mathrm{HCO}_{3}^{-}$secretion to counteract acid-induced mucosal damage (Takeuchi et al., 1999). Species differences may occur in the gastrointestinal functions of prostanoid receptors (Dey et al., 2006).

In cancer, $\mathrm{EP}_{3}$ receptors have been found to exert both facilitatory and inhibitory effects. In human colon cancer specimens, $\mathrm{EP}_{3}$ receptor mRNA was reduced by $28 \%$ compared with the normal colon mucosa (Shoji et al., 2004). Studies in the human colon cancer cell line HCA-7 showed that a selective agonist decreased viable cell numbers by $30 \%$, providing supportive evidence for $\mathrm{EP}_{3}$ receptor down-regulation playing a permissive role in colon carcinogenesis (Shoji et al., 2004). In a detailed study on individual mRNA splicing variants of the $\mathrm{EP}_{3}$ receptor, overexpression of each individual isoform decreased tumorigenic potential in cell lines and stably transfected human embryonic kidney 293 or HCT 116 cells exhibited decreased tumor growth in vivo (MaciasPerez et al., 2008). Analysis of second-messenger signaling revealed a $\mathrm{G}_{12}$-RhoA pathway for all three variants (Macias-Perez et al., 2008). $\mathrm{EP}_{3}$ receptors decreased aromatase activity in human adipose stromal cell lines, pointing to an inhibitory role in breast cancer (Richards and Brueggemeier, 2003). A role for $\mathrm{EP}_{3}$ receptors in tumor development is supported by other studies. In a model of tumor-stromal angiogenesis, tumor growth and angiogenesis were inhibited in $\mathrm{EP}_{3}(-/-)$ mice and by a selective antagonist (Amano et al., 2003). A role for $\mathrm{EP}_{3}$ receptors in up-regulating VEGF (Amano et al., 2003) has been confirmed with respect VEGF and VEGF receptor expression (Taniguchi et al., 2008; Amano et al., 2009). In addition, a contributory role for $\mathrm{EP}_{3}$ receptors has been suggested in oral squamous cancer cell growth (Hoshikawa et al., 2009).

Studies on edema formation have yielded tissuespecific results. In edema formation in rat and mouse skin, $\mathrm{EP}_{3}$ receptor stimulation potently inhibited the response to zymosan-activated serum and plateletactivating factor (Ahluwalia and Perretti, 1994). $\mathrm{PGE}_{2}-$ induced mouse paw edema was $\mathrm{EP}_{3}$ receptor-mediated (Claudino et al., 2006). In the rat adjuvant arthritis model, synoviocytes expressed the $\mathrm{EP}_{3} \mathrm{~B}$ isoform, which mediated FL-6 release (Kurihara et al., 2001). $\mathrm{EP}_{3}$ antagonists are also reported to be active in standard models of pain and inflammation (Jones et al., 2009).

Inflammatory cell studies seem largely restricted to $\mathrm{T}$ cells and mast cells. Prolactin stimulates Ig and cytokine release from $\mathrm{T}$ cells and $\mathrm{PGE}_{2}$ enhances prolactin transcription via $\mathrm{EP}_{3}$ and $\mathrm{EP}_{4}$ receptors (Gerlo et al., 2004). $\mathrm{EP}_{3}$ receptors also regulate expression and release of matrix metalloproteinase-9 in early T cells (Zeng et al., 1996). Both of these studies relied on cultured cell lines. The influence of $\mathrm{EP}_{3}$ receptors on mast-cell function seem of greater pathophysiological significance. The first studies employed mouse bone marrow derived mast cells, which expressed all four EP receptor subtypes (Nguyen et al., 2002). However, only $\mathrm{EP}_{3}$ receptors were involved in potentiation of antigen-induced degranulation and IL-6 production (Nguyen et al., 2002). The importance of $\mathrm{EP}_{3}$ receptors in allergic airway inflammation was demonstrated by 1) suppression of eosinophil infiltration and antigen-induced mediator release by the $\mathrm{EP}_{3}$ agonist ONO-AE-248 (Fig. 4) and 2) development of a more prominent eosinophil and mononuclear cell infiltrate and increased cytokine release in $\mathrm{EP}_{3}(-/-)$ mice (Kunikata et al., 2005). In dermal mast cells, $\mathrm{EP}_{3}$ receptor-induced degranulation occurred only in older mice, suggesting reprogramming with age (Nguyen et al., 2005). Studies in human mast cells provide at least partial confirmation in that $\mathrm{PGE}_{2}$ potentiates IgE-mediated histamine release via $\mathrm{EP}_{3}$ receptors but also $\mathrm{EP}_{1}$ receptors (Wang and Lau, 2006). Studies on the recruitment of mast cell progenitors showed that $\mathrm{EP}_{3}$ receptors are also chemoattractant for mast cells (Weller et al., 2007).

Beyond $\mathrm{EP}_{3}$ receptor-mediated down-regulation of cutaneous mast cell function and a potential role in type 1 allergies such as urticaria, $\mathrm{EP}_{3}$ receptors have also been implicated in keratinocytes and indirectly in atopic dermatitis. This is based on increased neutrophin 4 expression in keratinocytes, a factor that may participate in the hyperinnervation that occurs in atopic dermatitis (Kanda et al., 2005). Although the choice of pharmacological agents chosen for these studies was not ideal for unambiguously distinguishing $\mathrm{EP}_{3}$ from $\mathrm{EP}_{1}$ involvement, the use of antisense oligonucleotides provided 
strong evidence for responses exclusively mediated by $\mathrm{EP}_{3}$ receptors (Kanda et al., 2005). In addition, $\mathrm{EP}_{3}$ receptors were shown to inhibit the growth of human primary keratinocytes (Konger et al., 2005b). Three $\mathrm{EP}_{3}$ mRNA splicing variants were expressed in the intact human epidermis and keratinocytes. Predominant expression in the proliferative zone of the intact epidermis further supported involvement in regulating proliferation (Konger et al., 2005b).

Very diverse activities have been attributed to $\mathrm{EP}_{3}$ receptors in the lung. $\mathrm{EP}_{3}$ agonists constrict the human pulmonary artery (Qian et al., 1994). GGE $_{2}$-induced sensory nerve activation, as assessed by depolarization of vagus nerves, was found to be $\mathrm{EP}_{3}$ receptor-mediated (Maher et al., 2009). Apnea in human neonates has been related to increased $\mathrm{PGE}_{2}$ release and $\mathrm{EP}_{3}$-mediated modulation of respiratory neurons in the brainstem (Hofstetter et al., 2007). $\mathrm{EP}_{3}$ receptors may not only participate in infection (sepsis)-induced apnea (Hofstetter et al., 2007) but also may contribute to mortality caused by infection with Streptococcus pneumoniae, according to $\mathrm{EP}_{3}$ gene deletion studies (Aronoff et al., 2009). The $\mathrm{EP}_{3}$ agonist GR $63799 \mathrm{X}$ induced S-phase arrest and inhibited fibroblast growth (Sanchez and Moreno, 2006), which is possibly relevant to fibrotic disease of the lungs and fibrosis in general.

Prostaglandins are very important in uterine function and reproduction. $\mathrm{EP}_{3}$ receptors contract the myometrium (Senior et al., 1991). Two $\mathrm{EP}_{3}$ receptor isoforms were found to be expressed in the human uterus, which signaled via $G_{i}$ and MAP kinase (Kotani et al., 2000). $\mathrm{EP}_{3}$ receptors have also been implicated in cervical ripening and misoprostol is used clinically for labor induction and cervical ripening (Sanchez-Ramos et al., 1997).

c. Gene deletion studies. Two independent lines of $\mathrm{EP}_{3}(-/-)$ mice have been generated (Fleming et al., 1998; Ushikubi et al., 1998). The progeny of $\mathrm{EP}_{3}(-/-)$ mice were normal in all respects. Mice were born at the anticipated Mendelian frequency, survived normally, and were observed to be normal. Histological examination of tissues from $\mathrm{EP}_{3}$ receptor-deficient mice revealed no pathological changes (Fleming et al., 1998). Fertility and reproduction were normal in all respects (Fleming et al., 1998; Ushikubi et al., 1998). Nonsteroidal antiinflammatory drugs have been known as antipyretics, and the generation of prostanoid receptor knockout mice has enabled further insight into the pharmacology of febrile responses. These investigations were guided by the original proposal that $\mathrm{PGE}_{1}$ acted as a central mediator of fever (Milton and Wendlandt, 1970). Mice lacking $\mathrm{EP}_{3}$ receptors failed to mount a febrile response to $\mathrm{PGE}_{2}$ or to IL- $1 \beta$ or LPS (Ushikubi et al., 1998). In contrast, $\mathrm{PGE}_{2}$ induced fever in $\mathrm{EP}_{1}(-/-), \mathrm{EP}_{2}(-/-)$, and $\mathrm{EP}_{4}(-/-)$ mice (Ushikubi et al., 1998). After these studies on body temperature using a rectal probe (Ushikubi et al., 1998), febrile responses and thermoregulation in $\mathrm{EP}_{1}(-/-)$ and $\mathrm{EP}_{3}(-/-)$ mice were studied by telemetric measurement of core temperature (Oka et al., 2003). In this more systematic study, the $\mathrm{EP}_{1}$ receptor was implicated in the febrile response. The secondary phase of the hyperthermia to LPS was blunted in $\mathrm{EP}_{1}(-/-)$ mice, whereas only the initial hyperthermic phase was affected in $\mathrm{EP}_{3}(-/-)$ mice (Oka et al., 2003a). Agonist studies also implicated both $\mathrm{EP}_{1}$ and $\mathrm{EP}_{3}$ involvement in hyperthermia (Oka et al., 2003b).

$\mathrm{EP}_{3}$ receptors have also been implicated in urine production, osmolality, and bladder function. $\mathrm{EP}_{3}$ receptors are not essential for regulation of urinary osmolality, but in animals treated with indomethacin, urinary osmolality was increased in $\mathrm{EP}_{3}(+/+)$ but not $\mathrm{EP}(-/-)$ mice (Fleming et al., 1998). Enhanced bladder capacity and $\mathrm{PGE}_{2}$-induced bladder hyperactivity were reduced in mice lacking $\mathrm{EP}_{3}$ recepors (McCafferty et al., 2008). Thus, $\mathrm{EP}_{3}$ receptors may contribute to overactive bladder disorders. $\mathrm{EP}_{3}$ receptor deletion studies have shown that the $\mathrm{EP}_{3}$ receptor is essential for maintaining duodenal $\mathrm{HCO}_{3}^{-}$secretion and mucosal integrity (Takeuchi et al., 1999).

The majority of studies have implicated $\mathrm{EP}_{3}$ receptors in inflammation, pain, and associated events. Mice lacking $\mathrm{EP}_{3}$ receptors develop more pronounced allergic airway inflammation than wild-type or other knockout mice (Kunikata et al., 2005). Allergic inflammation was suppressed by an $\mathrm{EP}_{3}$ agonist (Kunikata et al., 2005). In complete contrast, $\mathrm{EP}_{3}$ receptor deletion has equally revealed proinflammatory roles. Systematic study of all EP knockout mice identified only the $\mathrm{EP}_{3}$ receptor as responsible for $\mathrm{PGE}_{2}$-induced mast cell activation and associated proinflammatory signaling pathways (Nguyen et al., 2002). Arachidonic acid-induced cutaneous microvascular exudation and edema was attenuated in $\mathrm{EP}_{3}(-/-)$ but not $\mathrm{EP}_{1}(-/-), \mathrm{EP}_{2}(-/-)$, or $\mathrm{EP}_{4}(-/-)$ mice (Goulet et al., 2004), Exudate formation is also partially mediated by $\mathrm{EP}_{3}$ receptors in the carrageenininduced pleurisy model (Yuhki et al., 2004). Additional studies on the lung have found that $\mathrm{EP}_{3}$ receptor deletion protects against severe $S$. pneumoniae infection (Aronoff et al., 2009) and virtually abolishes $\mathrm{PGE}_{2^{-}}$ induced depolarization of isolated vagus nerves (Maher et al., 2009). $\mathrm{EP}_{3}$ receptors have also been implicated in angiogenesis. Thus, in full-thickness skin wounds, $\mathrm{EP}_{3}$ receptor deletion delayed wound closure, re-epithelialization, and angiogenesis (Kamoshita et al., 2006). Accordingly, CD31 and VEGF expression were reduced (Kamoshita et al., 2006). COX-2 and $\mathrm{EP}_{3}$ receptors have also been found to be involved in acute herpetic pain: in contrast to $\mathrm{EP}_{3}(-/-)$ mice, the allodynia and hyperalgesia were not altered in $\mathrm{EP}_{1}(-/-), \operatorname{IP}(-/-)$, or TP(-/-) mice (Takasaki et al., 2005). Tumor progression was also attenuated in $\mathrm{EP}_{3}$-deficient mice (Shoji et al., 2005).

As a possible connection between inflammatory signaling and obesity, effects observed in $\mathrm{EP}_{3}$ receptor-deficient mice are of interest. The body weight illustration and histogram data are quite striking, the $\mathrm{EP}_{3}$-deficient mice be- 
ing clearly obese compared with wild-type littermate control mice (Sanchez-Alavez et al., 2007). Adult $\operatorname{EP}_{3}(-/-$ ) mice were feeding more frequently during the day and developed an obese phenotype on a normal fat diet. Increased locomotor activity did not affect the obesity. Obesity was accompanied by elevated leptin and insulin levels (Sanchez-Alavez et al., 2007).

The presence of functional $\mathrm{EP}_{3}$ receptors that potentiate platelet aggregation and inhibit adenylate cyclase has been recognized for some time (Matthews and Jones, 1993). Consistent with these findings, mice lacking $\mathrm{EP}_{3}$ receptors show an increased bleeding tendency and decreased susceptibility to thromboembolism (Ma et al., 2001). Finally, few studies on prostanoid receptor overexpression have been reported. In transgenic mice with cardiospecific $\mathrm{EP}_{3}$ receptor overexpression, increased calcineurin and NFAT activity with cardiac hypertrophy were found (Meyer-Kirchrath et al., 2009).

d. Agonists and antagonists. Phenoxy substitution (particularly $p$-halo-phenoxy) at C16 on the PGE template modestly promotes $\mathrm{EP}_{1}, \mathrm{EP}_{3}$, and $\mathrm{FP}$ agonism but markedly accentuates TP agonism. 16- $p$-Chlorophenoxy- $\omega$-tetranor $\mathrm{PGE}_{2}$ (ICI-80205) is a good example of these trends (Jones et al., 1982; Lawrence et al., 1992). 11-Deoxy-16-phenoxy $\mathrm{PGE}_{1}$ (MB-28767) shows reasonably good $\mathrm{EP}_{3}$-versus- $\mathrm{EP}_{1}$ selectivity but is still a moderately potent TP agonist (Banerjee et al., 1985; Lawrence and Jones, 1992; Lawrence et al., 1992; Boie et al., 1997). Sulprostone, in which the C1 carboxylate is converted to the acidic methylsulfonamide (Schillinger et al., 1979), has modest $\mathrm{EP}_{3}$-versus- $\mathrm{EP}_{1}$ selectivity and minimal TP agonism (Coleman et al., 1987; Coleman and Sheldrick, 1989). The combination of sulprostone and 17-phenyl $\mathrm{PGE}_{2}$ (Lawrence et al., 1992) has often been used to discriminate $\mathrm{EP}_{1}$ and $\mathrm{EP}_{3}$ receptors but is clearly not ideal. In a comprehensive structure-activity relationship study using guinea pig vas deferens and binding to mouse recombinant EP receptor subtypes, Shimazaki et al. (2000) showed that replacement of the C1-carboxylate in 13,14-didehydro-16-phenoxy $\mathrm{PGE}_{1}$ by a primary alcohol group (Fig. 4, compound 7b) results in only modest loss of agonist potency coupled with high $\mathrm{EP}_{3}$ selectivity; the C1-methyl ketone analog showed a similar profile.

Other routes to $\mathrm{EP}_{3}$-selective agonism have been explored. Deriving from misoprostol, SC-46275 (Fig. 4) is a highly potent and selective $\mathrm{EP}_{3}$ agonist (Savage et al., 1993) used in a limited number of investigations (Jones et al., 1998); the orientation of the 16-hydroxyl is opposite that of ONO-AE-259. Again, de-esterification may be required for full bioactivity. Finally, methylation of both 11- and 15-hydroxyls in $\mathrm{PGE}_{2}$ (ONO-AE-248; Fig. 4) imparts high $\mathrm{EP}_{3}$ selectivity (Okada et al., 2000; Suzawa et al., 2000), but potency is only modest (Jones et al., 2008).

The development of $\mathrm{EP}_{3}$ antagonists has recently been reviewed (Jones et al., 2009) and is therefore summa- rized only briefly. The acryloylsulfonamides L-798106 and L-826266 emerged from a combinatorial approach (Fig. 5) (Gallant et al., 2002; Belley et al., 2005). They are both highly lipophilic with slow onsets on certain isolated smooth muscle preparations (Jones et al., 2008). The related $\mathrm{EP}_{3}$ antagonist DG-041 (Heptinstall et al., 2008; Singh et al., 2009) is even more lipophilic (log $P=$ 7.67); affinity is maintained when the acryloyl unit is replaced by a heterocycle (Fig. 5, Hategan et al., 2009). More water-soluble antagonists are present within the DeCode Genetics series (O'Connell et al., 2009); one of these showed faster block than L-798106/L-826266 (Jones et al., 2011). An $\mathrm{EP}_{3}$ antagonist of a different class is represented by compound 49 (Fig. 5) (Asada et al., 2010); it showed good in vivo activity against $\mathrm{PGE}_{2^{-}}$ induced contraction of pregnant rat uterus.

e. Therapeutics. $\mathrm{EP}_{3}$ receptor pharmacology has successfully resulted in small-molecule therapeutics in current use, both of which contain misoprostol (Collins et al., 1985). Misoprostol is used for cervical ripening and labor induction (Sanchez-Ramos et al., 1997; Woodward and Chen, 2004). Misoprostol is effective gastric ulcer therapy (Rachmilewitz et al., 1986) and is combined with a nonsteroidal anti-inflammatory agent to ameliorate gastric irritation and ulceration and is commercially available as Arthrotec (G. D. Searle, Peapack, NJ) (Woodward and Chen, 2004). Misoprostol is a potent $\mathrm{EP}_{3}$ agonist but does not really possess adequate receptor selectively for modern therapeutic application. Given the complexity of PG-mediated effects (e.g., $\mathrm{EP}_{3}$ receptors and $\mathrm{EP}_{2} / \mathrm{EP}_{4}$ receptors having opposing effects on cAMP), a highly selective agent may be preferable for new uses. A decade of recent $\mathrm{EP}_{3}$ research has witnessed diverse findings. $\mathrm{EP}_{3}$ agonists protect against ischemic myocardial injury and reduced infarct size (Hohlfeld et al., 1997, 2000; Zacharowski et al., 1999), culminating in a study on transgenic mice with cardiospecific $\mathrm{EP}_{3}$ overexpression (Martin et al., 2005). Reduction in tumor development may be achieved with $\mathrm{EP}_{3}$ agonists (Amano et al., 2003; Macias-Perez et al., 2008), and down-regulation of $\mathrm{EP}_{3}$ receptors may enhance colon carcinogenesis in later stages (Shoji et al., 2004). Suppression of allergic inflammation by $\mathrm{EP}_{3}$ receptor activation has also been demonstrated (Kunikata et al., 2005). Finally, $\mathrm{EP}_{3}$ agonists may offer treatment for opiate withdrawal syndrome (Nakagawa et al., 1995). The potential of the $\mathrm{EP}_{3}$ agonist-based therapeutic options (Table 6) must be viewed from the standpoint that $\mathrm{EP}_{3}$ receptor stimulation may result in many pathophysiological events, as indicated by the potential uses of $\mathrm{EP}_{3}$ antagonists.

Potential therapeutic uses of $\mathrm{EP}_{3}$ receptor antagonists, identified and confirmed in animal models with compounds, are summarized in Table 7. These include pain and inflammation (Jones et al., 2009), type 1 allergy (Nguyen et al., 2002), lung infection (Aronoff et al., 2009), cough (Maher et al., 2009), overactive bladder 
TABLE 6

Potential therapeutic application of $\mathrm{EP}_{3}$ agonists

\begin{tabular}{|c|c|c|c|c|c|c|}
\hline Agonist & Route & Dose & Species & Experimental Model & Indication & Reference \\
\hline MB 28767 & Intravenous & $2 \mathrm{pmol} \cdot \mathrm{kg}^{-1} \cdot \min ^{-1}$ & Pig & Occlusion/reperfusion & Cardiac infarction & Hohlfeld et al., 2000 \\
\hline MB 28767 & Intracisternal & $\begin{array}{l}1 \text { fg-30 ng per } \\
\text { animal }\end{array}$ & Mouse & $\begin{array}{l}\text { Naloxone-induced } \\
\text { withdrawal jumping }\end{array}$ & $\begin{array}{l}\text { Opiate withdrawal } \\
\text { syndrome }\end{array}$ & Nakagawa et al., 1995 \\
\hline ONO-AE-248 & Subcutaneous & $10 \mu \mathrm{g} / \mathrm{kg}$ & Mouse & Ovalbumin induced asthma & Asthma & Kunikata et al., 2005 \\
\hline TEI-3356 & Intravenous & $1 \mu \mathrm{g} \cdot \mathrm{kg}^{-1} \cdot \mathrm{h}^{-1}$ & Rat & Occlusion/reperfusion & Cardiac infarction & Zacharowski et al., 1999 \\
\hline
\end{tabular}

TEI-3356, 5-(-7-hydroxy-6-(4-hydroxy-4-methyl-1-octenyl)bicyclo(3.3.0)oct-2-en-3-yl)pentanoic acid.

TABLE 7

Potential therapeutic application of $E P_{3}$ antagonists

\begin{tabular}{|c|c|c|c|c|c|c|}
\hline Antagonist & Route & Dose & Species & Experimental Model & Indication & Reference \\
\hline DG-041 & Intravenous & $10 \mathrm{mg} / \mathrm{kg}$ & Rat & Urinary bladder distension & Overactive bladder & Su et al., $2008 \mathrm{a}$ \\
\hline DG-041 & Oral & $0.1-100 \mathrm{mg} / \mathrm{kg}$ & Rat & $\begin{array}{l}\text { Ex vivo platelet aggregation; } \\
\text { bleeding time }\end{array}$ & Atherothrombosis & Singh et al., 2009 \\
\hline L-826266 & $\begin{array}{l}\text { Intraperiaqueductal } \\
\text { grey }\end{array}$ & $0.125 \mathrm{nM}-0.5 \mathrm{nM}$ & Mouse & Formalin induced hyperalgesia & Pain & Oliva et al., 2006 \\
\hline L-826266 & Intraplantar & $3-30 \mathrm{nmol}$ & Mouse & Paw-licking & Rheumatoid arthritis & Kassuya et al., 2007 \\
\hline ONO-AE3-240 & Subcutaneous & $3.30 \mathrm{mg} / \mathrm{kg}$ & Mouse & HSV-1 inoculation & Post-herpetic pain & Takasaki et al., 2005 \\
\hline ONO-AE3-240 & Subcutaneous & $50 \mathrm{nmol} /$ tumor & Mouse & Sarcoma 180 cell tumor & Cancer & Amano et al., 2003 \\
\hline
\end{tabular}

HSV-1, Herpes simplex virus 1.

(McCafferty et al., 2008; Su et al., 2008), hyperpyrexia (Ushikubi et al., 1998), and cancer (Amano et al., 2003). Most recently, $\mathrm{EP}_{3}$ antagonists have emerged as a new target for antiplatelet agents in atherothrombotic disease, without prolonged bleeding (Heptinstall et al., 2008; Singh et al., 2009).

\section{4. $\mathrm{EP}_{4}$ Receptors.}

a. Second messenger signaling. $\mathrm{EP}_{4}$ receptors are widely distributed (Narumiya et al., 1999). The pharmacologically defined $\mathrm{EP}_{4}$ receptor was originally designated $\mathrm{EP}_{2}$ (Honda et al., 1993; Bastien et al., 1994) until the authentic $\mathrm{EP}_{2}$ receptor was cloned (Regan et al., 1994b). Studies on second-messenger signaling demonstrated functional coupling to cAMP via $\mathrm{G}_{\mathrm{s}}$ (Narumiya et al., 1999). Certain $\mathrm{EP}_{4}$ receptor-mediated effects seem to exclusively employ the cAMP-PKA pathway (Southall and Vasko, 2001; Gray et al., 2004; Ziemann et al., 2006; Boniface et al., 2009). Direct comparison of $\mathrm{EP}_{4}$ and $\mathrm{EP}_{2}$ receptor signaling demonstrated that the functional coupling to cAMP seems less efficient for $\mathrm{EP}_{4}$ compared with the $\mathrm{EP}_{2}$ subtype (Fujino et al., 2002, 2005). The prospect of a second $\mathrm{EP}_{4}$ signaling pathway was realized when a PI3K signaling pathway was discovered coupled to the pertussis toxin-sensitive $G$ protein $G_{i}$ (Fujino et al., 2002, 2003, 2005; Fujino and Regan, 2005). This also provides a mechanism for limiting the cAMP response to $\mathrm{EP}_{4}$ stimulation (Fujino and Regan, 2006). It should be noted that several cAMP-independent signaling cascades have been reported for $\mathrm{EP}_{4}$ receptor activation (Fiebich et al., 2001; Pozzi et al., 2004; Mendez and LaPointe, 2005; Frias et al., 2007; George et al., 2007; Rao et al., 2007). Evidence for the $\mathrm{EP}_{4}$ signaling cascade PI3K-ERK-early growth response-1 (Fujino et al., 2003) seems operative in cell growth (Pozzi et al., 2004; Mendez and LaPointe, 2005; Frias et al., 2007; Rao et al., 2007). Serum-deprived apoptosis in Jurkat cells was reduced by $\mathrm{EP}_{4}$ receptors through $\mathrm{PI} 3 \mathrm{~K}$ and cAMP path- ways may be operative, as in $\mathrm{EP}_{4}$-mediated inhibition of apoptosis (Leone et al., 2007).

Epac signaling has received little attention from the standpoint of prostanoid receptor signal transduction. A recent study has indicated both PKA and Epac 1 signaling in rheumatoid synovial fibroblasts (Kojima et al., 2009) for both $\mathrm{EP}_{2}$ and $\mathrm{EP}_{4}$ receptors. Both $\mathrm{EP}$ receptor subtypes activate the small GTPase Rap 1 (Kojima et al., 2009). Rap was also involved in $\mathrm{EP}_{4}$-mediated brain natriuretic peptide expression (Qian et al., 2006). $\mathrm{EP}_{4}$ receptors have also been shown to be involved in COX-2 mRNA induction and stabilization and stimulation of translation (Faour et al., 2001; Martineau et al., 2004), p38 MAP kinase playing a key role. $\mathrm{EP}_{4}$ receptor activation of p38 MAP kinase has been found in certain cells: astrocytes, podocytes (Fiebich et al., 2001; Martineau et al., 2004), RAW 264.7 cells (Chen et al., 2006), Caco-2 cells (Leone et al., 2007), and colonic myofibroblasts (Hoang et al., 2007). Such an $\mathrm{EP}_{4}$ effect on p38 MAP kinase is not always observed, for example in tracheobronchial epithelial cells (Gray et al., 2004) and neonatal ventricular myocytes (Qian et al., 2006). In cardiac myocytes, $\mathrm{EP}_{4}$ receptor-mediated hypertrophy (Mendez and LaPointe, 2005) and BNP promoter regulation (Qian et al., 2006) both involve p42/44 MAP kinase. The potential complexities of $\mathrm{EP}_{4}$ activating signaling cascades is illustrated by the concomitant activation of $\mathrm{PKC}$ in some instances (Fiebich et al., 2001; Chen et al., 2006).

b. Distribution and biological functions. $\mathrm{EP}_{4}$ receptors were originally pharmacologically characterized predominantly from studies on smooth muscle (Coleman et al., 1994a). Thereafter, $\mathrm{EP}_{4}$ receptor-mediated effects on smooth muscle tone have received little attention. $\mathrm{EP}_{4}$ receptors mediate vasorelaxation of pulmonary arterial veins but produce no effect on pulmonary arteries (Foudi et al., 2008). $\mathrm{EP}_{4}$ receptors, however, do not play 
an entirely passive role in the human pulmonary artery: COX-2 induction by bradykinin in human pulmonary arterial smooth muscle cells involves cAMP response element activation by $\mathrm{EP}_{4}$ and $\mathrm{EP}_{2}$ agonists (Bradbury et al., 2003). In the rat aorta, $\mathrm{EP}_{4}$ receptor-mediated vasorelaxation was endothelium-dependent and involved endothelial nitric-oxide synthase and cGMP (Hristovska et al., 2007). The most therapeutically significant study demonstrated $\mathrm{EP}_{4}$ receptor-mediated vasodilation of the human middle cerebral and meningeal arteries and obviated the potential clinical utility of $\mathrm{EP}_{4}$ antagonists for treating migraine (Davis et al., 2004; Maubach et al., 2009). $\mathrm{EP}_{4}$ overexpression in atherosclerotic plaque results in an unstable phenotype prone to inflammation and instability (Cipollone et al., 2005). Endothelial cell migration and angiogenesis in vivo are also produced by $\mathrm{EP}_{4}$ receptor activation (Rao et al., 2007; Jain et al., 2008).

An interesting aspect of $\mathrm{EP}_{4}$ cardiovascular biology is its potential involvement in cardiac hypertrophy. $\mathrm{EP}_{4}$ receptor activation produces cardiomyocyte hypertrophy, as measured by increased protein synthesis (Mendez and LaPointe, 2005; Miyatake et al., 2007; He at al., 2010), cell size and surface area (Frias et al., 2007; Miyatake et al., 2007; He et al., 2010), re-expression of fetal genes, and activation of hypertrophic marker genes such as BNP (Qian et al., 2006; Miyatake et al., 2007; He et al., 2010). This was confirmed in living animals, where cardiospecific $\mathrm{EP}_{4}$ receptor deletion resulted in decreased hypertrophy and fibrosis after experimental myocardial infarction (Qian et al., 2008). The transcription factor STAT3 was activated and correlated with hypertrophy and fibrosis (Frias et al., 2007; Qian et al., 2008). Cardiac function, paradoxically, was worsened in hearts lacking $\mathrm{EP}_{4}$ receptor expression (Qian et al., 2008), which actually correlates with $\mathrm{EP}_{4}$ protection from ischemia-reperfusion injury (Xiao et al., 2004).

The ductus arteriosus is a shunt in the fetus between the pulmonary artery and the aorta. Closure of the ductus arteriosus in the newborn is vital to prevent pulmonary hypertension-induced lung edema and congestive heart failure. The $\mathrm{EP}_{4}$ receptor is critical for remodeling of the ductus arteriosus at birth (Nguyen et al., 1997). Likewise, $\mathrm{EP}_{4}$ agonists reopen the ductus arteriosus in neonates dependent on placental oxygenation (Momma et al., 2005). Analogous to some extent, the uterine cervix also plays a crucial role in pregnancy; closed during gestation, soft and dilated during labor. $\mathrm{PGE}_{2}$ has been used for inducing cervical ripening for many years (Woodward and Chen, 2004), and the changes that occur resemble those that are observed in physiological ripening. $\mathrm{EP}_{4}$ receptor expression has been shown to be maximum at parturition in rats (Chien and Macgregor, 2003) and has been implicated in LPS-induced cervical ripening (Fukuda et al., 2007). Glycosaminoglycan biosynthesis is an important part of cervical ripening, and $\mathrm{EP}_{4}$ receptor stimulation produces this effect in human cervical fibroblasts via a PKA-independent pathway (Schmitz et al., 2001).

Results on $\mathrm{EP}_{4}$ receptor involvement in mucus secretion are mixed. $\mathrm{EP}_{4}$ and $\mathrm{EP}_{1}$ receptors evoke mucin exocytosis from central mucous cells (Ohnishi et al., 2001), whereas an $\mathrm{EP}_{4}$ agonist inhibited LPS-induced mucus secretion from airway epithelial cells (Hattori et al., 2008). $\mathrm{PGE}_{2}$ was claimed to protect guinea pig gastric mucosal cells from ethanol-induced apoptosis (Hoshino et al., 2003): set against this finding, the $\mathrm{EP}_{4}$ antagonist (S)-4-(1-(5-chloro-2-(4-fluorophenyoxy)benzamido)ethyl)benzoic acid (CJ-42794) did not damage the rat gastric mucosa or worsen the response to aspirin or stress (Takeuchi et al., 2007). Cytoprotective duodenal $\mathrm{HCO}_{3}^{-}$secretion is also $\mathrm{EP}_{4}$ receptor-mediated in rats (Aoi et al., 2004) and humans (Larsen et al., 2005).

There is an extensive volume of literature on PGs and bone formation, and $\mathrm{EP}_{4}$ agonists occupy a prominent place. $\mathrm{EP}_{4}$ receptors mediate not only bone formation but also bone resorption, as indicated in $\mathrm{EP}_{4}(-/-)$ mice (Miyaura et al., 2000). Nevertheless, a litany of reports shows $\mathrm{EP}_{4}$-mediated bone formation, augmentation of bone morphogenetic protein-induced bone mass, and beneficial effects on fracture healing. These findings suggest that, physiologically, $\mathrm{EP}_{4}$ receptors favor resorption, but exogenously administered $\mathrm{EP}_{4}$ agonists have an anabolic effect on bone formation. It may be speculated that exogenous $\mathrm{EP}_{4}$ agonists locate receptors not under $\mathrm{PGE}_{2}$ regulation under normal physiological circumstances. The potential therapeutics associated with $\mathrm{EP}_{4}$ and $\mathrm{EP}_{2}$ agonists for treating bone loss and accelerating bone repair have been reviewed ( $\mathrm{Li}$ et al., 2007; Graham et al., 2009). It should also be noted that findings in vitro may not transition into in vivo studies; thus, although $\mathrm{EP}_{4}$ receptors are essential for anabolic responses to $\mathrm{PGE}_{2}$, in osteoblasts, they are not essential for bone remodeling in living animals (Gao et al., 2009). In quite marked contrast, the $\mathrm{EP}_{4}$ antagonist $N$-(((2-(4(2-ethyl-4,6-dimethyl-1H-imidazo(4,5-c)pyridin-1-yl) phenyl)ethyl)amino)carbonyl)-4-methylbenzenesulfonamide (CJ-023,423) reduced bone destruction in the rat adjuvant-induced arthritis model (Okumura et al., 2008). In mouse collagen-induced arthritis, the $\mathrm{EP}_{4}$ antagonist ONO-AE3-208 (Fig. 5) did not alter the arthritis in wild-type mice (Honda et al., 2006). These results indicate that $\mathrm{EP}_{4}$ receptor participation in bone remodeling is disease-model specific.

Endochondral bone formation involves chondrocytes as well as coordinated bone formation and mineralized matrix resorption by osteoblasts and osteoclasts. In the growth plate, chondrocytes undergo a maturation process. Resting chondrocytes transition into proliferating chondrocytes, which express type II collagen mRNA and synthesize proteoglycan. Chondrocytes then mature into hypertrophic cells characterized by a 5 - to 10 -fold increase in cell volume. Terminal differentiation is associated with expression of genes associated with calcifica- 
tion of bone matrix, such as osteocalcin. Terminally differentiated chondrocytes undergo apoptosis, and the calcified cartridge left behind provides a template for primary bone formation (Cheung et al., 2003). An understanding of $\mathrm{PG}$ involvement in each phase of chondrocyte maturation is of significance for osteoarthritis and rheumatoid arthritis. $\mathrm{EP}_{4}$ receptor stimulation alone did not up-regulate type II collagen expression or increase proteoglycan in rat growth plate chondrocytes; concomitant $\mathrm{EP}_{2}$ receptor activation was required (Miyamoto et al., 2003). Other murine chondrocyte studies are not entirely in agreement (Clark et al., 2005; Brochhausen et al., 2006), these studies using more physiological concentrations. The concentrations of $\mathrm{PGE}_{2}$ in studies on human chondrocytes are also sufficiently high to be in the nonselective range.

Fibroblast-like synoviocytes are important in the pathogenesis of rheumatoid arthritis, where they proliferate and secrete enzymes involved in joint degradation and cytokine/chemokine production. In human synovial fibroblasts, IL- $1 \alpha$ (Yoshida et al., 2001) and IL-1 $\beta$ (Faour et al., 2001) effects are mediated by $\mathrm{PGE}_{2}$ release. $\mathrm{EP}_{2}$ and $\mathrm{EP}_{4}$ receptors are consistently expressed in human synovial fibroblasts (Yoshida et al., 2001; Mathieu et al., 2008; Kojima et al., 2009). IL-1 $\beta$-induced up-regulation of COX-2 was ascribed to interaction of released $\mathrm{PGE}_{2}$ with $\mathrm{EP}_{4}$ receptors (Faour et al., 2001). IL-1 $\beta$ also increases $\mathrm{PGE}_{2}$ production in human tendon fibroblasts, which mediates down-regulation of type 1 collagen via $\mathrm{EP}_{4}$ receptors, which in turn may disturb tendon homeostasis (Thampatty et al., 2007).

Studies on leukocyte $\mathrm{EP}_{4}$ receptor function have been largely restricted to mononuclear cells. In mouse peritoneal neutrophils, $\mathrm{EP}_{4}$ and $\mathrm{EP}_{2}$ receptors suppress $\mathrm{TNF} \alpha$ production, and $\mathrm{EP}_{2}$ receptors augment IL-6 production (Yamane et al., 2000). In human eosinophils, $\mathrm{EP}_{4}$ mRNA was found to be significantly higher than $\mathrm{EP}_{2}$ (Mita et al., 2002). In human blood monocytes, $\mathrm{EP}_{2}$ and $\mathrm{EP}_{4}$ receptors up-regulated $\mathrm{C}-\mathrm{C}$ chemokine receptor 7 mRNA, which is essential for migration to secondary lymphoid tissues (Côté et al., 2009). In a further study on human macrophages, only $\mathrm{EP}_{4}$ receptors were expressed and therefore considered to mediate $\mathrm{PGE}_{2}$ inhibition of macrophage inhibitory proteins $1 \alpha$ and $1 \beta$, IL-8, monocyte chemotactic protein-1, and IL-10 release (Takayama et al., 2002).

$\mathrm{PGE}_{2}$ was also found to be a key factor for increased $\mathrm{C}-\mathrm{C}$ chemokine receptor $7 \mathrm{mRNA}$ expression in monocyte-derived dendritic cells (Scandella et al., 2002), although the relative importance of $\mathrm{EP}_{4}$ and $\mathrm{EP}_{2}$ receptors is uncertain because of indeterminate pharmacological definition. $\mathrm{PGE}_{2}$ is regarded as essential for the development of a migratory phenotype of human dendritic cells, and this is ascribed to $\mathrm{EP}_{4}$ and $\mathrm{EP}_{2}$ receptor mediation (Luft et al., 2002; Harizi et al., 2003; Legler et al., 2006; McIlroy et al., 2006). Polarization into Th-2 helper cells seems to involve both $\mathrm{EP}_{4}$ and $\mathrm{EP}_{2}$ receptors
(Kubo et al., 2004; McIlroy et al., 2006; Krause et al., 2007). The choice and concentrations of the pharmacological "tools" in these studies was not always ideal, and further investigation would be worthwhile. $\mathrm{EP}_{4}$ receptors were shown to initiate cutaneous immune responses by promoting Langerhans cell maturation and migration, in a comprehensive study employing $\mathrm{EP}_{4}(-/-)$ mice and selective $\mathrm{EP}_{4}$ agonist and antagonist compounds (Kabashima et al., 2003b).

T-cell functions are also regulated by $\mathrm{PGE}_{2}$ and $\mathrm{EP}_{4}$ receptor activation. $\mathrm{PGE}_{2}$ was shown to suppress Th1 and Th2 T-helper cell activity. Both $\mathrm{EP}_{4}$ and $\mathrm{EP}_{2}$ receptors acted additively to suppress Th1 cell proliferation and IFN- $\gamma$ release. In Th2 T-helper cells, independent $\mathrm{EP}_{2}$ and $\mathrm{EP}_{4}$ receptor activation virtually abolished proliferation, but combined $\mathrm{EP}_{2} / \mathrm{EP}_{4}$ agonism was most effective in inhibiting IL-4 production (Okano et al., 2006). Some studies have implicated $\mathrm{EP}_{4}$ and $\mathrm{EP}_{2}$ receptors in development of the Th17 phenotype from naive T cells. Th17 T cells are distinct from Th1 and Th2 subsets. Human Th17 T-helper cell differentiation is controlled by the retinoic acid receptor-related orphan receptor- $\gamma \mathrm{t}$, and $\mathrm{PGE}_{2}$ seems to synergize with IL- $1 \beta$ and IL-23 to up-regulate retinoic acid receptor-related orphan receptor- $\gamma \mathrm{t}$ and down-regulate $\mathrm{T}$ cell-specific $\mathrm{T}$ box transcription factor, IFN- $\gamma$ and the anti-inflammatory cytokine IL-10 (Boniface et al., 2009; Napolitani et al., 2009). No role for $\mathrm{EP}_{1}$ or $\mathrm{EP}_{3}$ receptors was apparent. A clear role for $\mathrm{EP}_{2}$ receptors was established, but the choice of $\mathrm{EP}_{4}$ agonists and the high concentrations used cannot rule out $\mathrm{EP}_{2} / \mathrm{EP}_{4}$ mutual inhibitory activity, $\mathrm{EP}_{2} / \mathrm{EP}_{4}$ synergism, or other nonspecific activity. In an in vivo transplantation model, a combination of $\mathrm{EP}_{2}, \mathrm{EP}_{3}$, and $\mathrm{EP}_{4}$ agonists was needed to match the immunosuppressive effect of $\mathrm{PGE}_{2}$ (Fujimoto et al., 2005). A singular $\mathrm{EP}_{4}$ receptor-mediated event was found in HIV-1-infected $\mathrm{T}$ cells with respect to pro-viral DNA activation (Dumais et al., 1998). Based on Pt ger $4\left(\mathrm{EP}_{4}\right)$ behaving as a strongly expressed, delayed early gene that inhibits $\mathrm{B}$ cell proliferation (Murn et al., 2008), $\mathrm{EP}_{4}$ receptors could represent a novel target for treatment of $\mathrm{B}$ cell malignancies.

Numerous reports have linked $\mathrm{EP}_{4}$ receptor activation to cancer. $\mathrm{EP}_{4}$ receptors promote cancer in many dimensions: cell proliferation (Cherukuri et al., 2007; Zheng et al., 2009), cell survival (George et al., 2007), invasiveness (Spinella et al., 2004; Pan et al., 2008), angiogenesis (Jain et al., 2008), migration (Kim et al., 2010), and tumor metastasis (Ma et al., 2006; Yang et al., 2006). $\mathrm{PGE}_{2}$ produces proliferation of colon cancer cell lines H-29 (Chell et al., 2006) and HCA7 (Cherukuri et al., 2007). This is consistent with the idea that $\mathrm{EP}_{4}$ is the most abundant transcript in both H-29 and HCA7 cells, which also biosynthesize $\mathrm{PGE}_{2}$ via COX-2 (Doherty et al., 2009). $\mathrm{EP}_{4}$ receptors stably transfected into HT-29 cells promoted anchorage-independent growth and increased resistance to apoptosis and the formation of fluid-filled cysts (Hawcroft et al., 2007). Transfection of 
$\mathrm{EP}_{4}$ receptors into a human adenoma cell line (RG/C2) also introduces anchorage-independent growth (Chell et al., 2006). $\mathrm{PGE}_{2}$ also caused $\mathrm{EP}_{4}$-mediated cell growth in untransfected RG/C2 adenoma cells (Chell et al., 2006). COX-2 (Gustafsson et al., 2007; Yuan et al., 2008; Doherty et al., 2009) and $\mathrm{EP}_{4}$ receptor expression are increased in colorectal cancer (Chell et al., 2006), although the latter result was not confirmed (Gustafsson et al., 2007). Animal models provide evidence for a major role for $\mathrm{EP}_{4}$ receptors in the development of colon cancer (Mutoh et al., 2002; Kitamura et al., 2003b; Yang et al., 2006). $\mathrm{EP}_{4}$ receptors have been implicated in many forms of cancer: these include lung (Han et al., 2007; Zheng et al., 2009), upper urinary tract (Miyata et al., 2005), stomach (Okuyama et al., 2002), prostate (Jain et al., 2008), breast (Timoshenko et al., 2003; Ma et al., 2006; Pan et al., 2008; Robertson et al., 2008; Subbaramaiah et al., 2008), cervix (Sales et al., 2001; Muller et al., 2006; Oh et al., 2009), ovary (Spinella et al., 2004), and nonmelanoma skin cancer (Lee et al., 2005).

Prostanoid $\mathrm{EP}_{4}$ receptors have been extensively implicated in mediating hyperalgesia and allodynia (Jones et al., 2009). All EP subtypes are expressed in sensory neurons, but $\mathrm{EP}_{4}$ may be regarded as the most important because it causes sensitization (Southall and Vasko, 2001) and is exclusively expressed in a subset of primary sensory dorsal root ganglia, which increases in subchronic inflammation (Lin et al., 2006). $\mathrm{EP}_{4}$ receptors protect against NMDA-induced acute excitotoxicity (Ahmad et al., 2005). A dichotomy of effects was found with respect to $\mathrm{EP}_{4}$ receptors in Alzheimer's disease. $\mathrm{PGE}_{2}$ was found to stimulate amyloid- $\beta$ peptide production via $\mathrm{EP}_{4}$ receptor internalization (Hoshino et al., 2009). However, cell death produced by $\beta$-amyloid was attenuated by $\mathrm{EP}_{4}$ and $\mathrm{EP}_{2}$ receptor stimulation (Echeverria et al., 2005).

c. Gene deletion studies. Two lines of systemic-null $\mathrm{EP}_{4}$-deficient mice were generated independently (Nguyen et al., 1997; Segi et al., 1998). Most $\mathrm{EP}_{4}$-deficient mice on a C57BL/6 background die within 3 days after birth because of marked pulmonary congestion and heart failure due to a patent ductus arteriosus. It is well known that administration of indomethacin to maternal mice during late pregnancy induces closure of the ductus in wild-type mice, which led to the proposal that endogenous PGs maintains the ductus open. However, indomethacin treatment did not induce closure of the ductus in $\mathrm{EP}_{4}$-deficient fetuses, indicating that a PG-independent dilatory mechanism comes into play in the absence of $\mathrm{EP}_{4}$. These findings led the authors to suggest that opening of this vessel during the embryonic period is mediated by the $\mathrm{PGE}_{2}-\mathrm{EP}_{4}$ signaling and that in its absence, the compensatory dilatory mechanism is mobilized, and this mechanism continues to maintain the vessel open after the birth, resulting in a paradoxical patent ductus arteriosus in the $\mathrm{EP}_{4}$-deficient mice. However, in contrast to this general idea on the patent ductus arteriosus phenotype of $\mathrm{EP}_{4}(-/-)$ mice,
Trivedi et al. (2006) suggested an alternative possibility that the $\mathrm{PGE}_{2}-\mathrm{EP}_{4}$ signaling functions close, not open, the ductus. Their suggestion was based on their findings that COX-2 is induced in the ductus around term; that the loss of COX-2 or long-term treatment with COX inhibitors leads to its opening, not closure; and that the COX-2 expression in the ductus around term was attenuated in $\mathrm{EP}_{4}$-deficient mice.

Survival of $\mathrm{EP}_{4}$-deficient mice can be improved using the mixed genetic background $\mathrm{C} 57 \mathrm{BL} / 6$ and $129 \mathrm{~s} / \mathrm{v}$. Therefore, colonies of $\mathrm{EP}_{4}(-/-)$ mice are maintained by intercrossing surviving $\mathrm{EP}_{4}(-/-)$ mice, and progenies of these colonies are used with progenies of the littermate wild-type mice as a control. Using these mice, functions of $\mathrm{EP}_{4}$ in several physiological processes have been examined, one being that in bone metabolism. Osteoclasts develop from precursor cells of the macrophage lineage in the bone microenvironment. Factors such as PTH, vitamin D, IL-1, and IL- 6 act on osteoblasts to induce the synthesis of RANKL, which in turn stimulates the formation of mature osteoclasts from hematopoietic precursors through cell-cell interaction. These factors induce COX-2 expression in osteoblasts, and their induction of osteoclast differentiation is inhibited, at least in part, by aspirin-like drugs; such inhibition is reversed by the addition of $\mathrm{PGE}_{2}$, implicating $\mathrm{PGE}_{2}$ in this process (Tai et al., 1997). Sakuma et al. (2000) and Miyaura et al. (2000) examined the identity of the EP subtype responsible for mediating this action of $\mathrm{PGE}_{2}$. Sakuma et al. (2000) found that $\mathrm{PGE}_{2}$-induced osteoclast formation was impaired in cocultures of osteoblasts from $\mathrm{EP}_{4}$ deficient mice and osteoclast precursors from the spleen of wild-type mice. IL- $1 \beta$, TNF- $\alpha$, and basic fibroblast growth factor also failed to induce osteoclast formation in these cultures. Miyaura et al. (2000) added $\mathrm{PGE}_{2}$ to cultures of parietal bone from mice deficient in each of the EP subtypes, as well as from wild-type mice, and examined bone resorption by measuring the release of $\mathrm{Ca}^{2+}$ into the medium. They found that the induction of bone resorption by $\mathrm{PGE}_{2}$ was greatly impaired, whereas bone resorption in response to dibutyryl cAMP was unaffected, in bone from $\mathrm{EP}_{4}$-deficient mice. These studies unequivocally established a role for $\mathrm{EP}_{4}$ receptors in the induction of osteoclast differentiation factor and in $\mathrm{PGE}_{2}$-dependent bone resorption. On the other hand, $\mathrm{Li}$ et al. (2000) showed that the osteoclastogenic response to $\mathrm{PGE}_{2}, \mathrm{PTH}$, or 1,25-dihydroxyvitamin $\mathrm{D}$ in vitro was impaired in cultures of cells derived from $\mathrm{EP}_{2}$-deficient mice. These findings probably reflect redundant roles of the two relaxant PGE receptor subtypes. In addition to bone resorption, systemic administration of $\mathrm{PGE}_{2}$ has long been known to induce bone formation in vivo. To examine the identity of the EP receptor in this process, Yoshida et al. (2002) infused $\mathrm{PGE}_{2}$ into the periosteal region of the femur of wild-type mice or mice-deficient in each EP subtype with a miniosmotic pump and found that, after 6 weeks, $\mathrm{PGE}_{2}$ induced extensive callus for- 
mation on the femur at the site of infusion in wild-type as well as $\mathrm{EP}_{1^{-}}, \mathrm{EP}_{2^{-}}$, and $\mathrm{EP}_{3^{-}}$-deficient mice but not in $\mathrm{EP}_{4}$-deficient mice. Infusion of an $\mathrm{EP}_{4}$-selective agonist, but not those specific for other EP subtypes, consistently induced bone formation in wild-type mice with increased density of both osteoblasts and osteoclasts. These findings suggest that $\mathrm{EP}_{4}$ is responsible for both bone resorption and bone formation induced by $\mathrm{PGE}_{2}$ and that activation of $\mathrm{EP}_{4}$ in situ integrates these two actions for bone remodeling.

Given the marked induction of COX-2 and high production of $\mathrm{PGE}_{2}$ in the heart during myocardial infarction and preferential expression of $\mathrm{EP}_{4}$ among EPs in this organ, Xiao et al. (2004) subjected $\mathrm{EP}_{4}$-deficient mice to an ischemia-reperfusion model by ligation of the left anterior descending coronary artery for $1 \mathrm{~h}$, followed by 24 -h reperfusion. They found that $\mathrm{EP}_{4}$-deficient mice developed larger infarct sizes than wild-type mice and that, conversely, administration of ONO-4819CD (Xiao et al., 2004), an $\mathrm{EP}_{4}$ agonist, administered $1 \mathrm{~h}$ before or $50 \mathrm{~min}$ after occlusion reduced the infarct size in wildtype mice. Based on these findings, the authors suggested that $\mathrm{EP}_{4}$ exerts cardioprotective actions under ischemia-reperfusion conditions in the heart. Another study using $\mathrm{EP}_{4}$-deficient mice in cardiovascular diseases concerned its role in atherosclerosis. Because macrophages that accumulate in early lesions of atherosclerosis are capable of producing a large amount of $\mathrm{PGE}_{2}$, and cells in atherosclerotic plaques express $\mathrm{EP}_{2}$ and $\mathrm{EP}_{4}$ among EP subtypes, Babaev et al. (2008) generated chimera mice in which fetal liver cells from either $\mathrm{EP}_{2}(-/-)$ or $\mathrm{EP}_{4}(-/-)$ mice were transplanted into lethally irradiated LDLR(-/-) mice. They then fed these mice with western chow for 8 weeks to develop atherosclerosis. They found that atherosclerotic lesions were significantly smaller in mice transplanted with cells of $\mathrm{EP}_{4^{-}}$ deficient mice $\left[\mathrm{EP}_{4}(-/-) \rightarrow \operatorname{LDLR}(-/-)\right]$ than either $\mathrm{WT} \rightarrow \operatorname{LDLR}(-/-)$ or $\mathrm{EP}_{2}(-/-) \rightarrow \operatorname{LDLR}(-/-)$ mice with significantly more apoptotic cells in the lesions. Further analysis revealed that macrophages from $\mathrm{EP}_{4}(-/-)$ chimeras are more sensitive to apoptotic stimuli, which may derive from attenuated PI3K and nuclear factor- $\kappa \mathrm{B}$ signaling in this line. These findings have led the authors to suggest that the macrophage $\mathrm{EP}_{4}$ signaling may be a target for suppressing development of atherosclerosis.

Studies on $\mathrm{EP}_{4}$-deficient mice also revealed a variety of actions of this EP subtype in immune inflammation. One action is on dendritic cells. Kabashima et al. (2003b) found that migration of Langerhans cells in the skin to draining lymph nodes on hapten application is impaired in $\mathrm{EP}_{4}(-/-)$ mice, and this impairment can be mimicked by treatment of animals with an $\mathrm{EP}_{4}$-selective antagonist. Consequently, contact hypersensitivity to the hapten was suppressed in $\mathrm{EP}_{4}$-deficient mice. Further analysis revealed that the $\mathrm{PGE}_{2}-\mathrm{EP}_{4}$ signaling facilitates mobilization, migration, and maturation of Langerhans cells after initial antigen application. Honda et al. (2006) found that $\mathrm{EP}_{4}$ also plays a role in collagen-induced arthritis. In this model, the $\mathrm{PGI}_{2}$-IP signaling and the $\mathrm{PGE}_{2}$ signaling through $\mathrm{EP}_{2}$ and $\mathrm{EP}_{4}$ additively mediate joint inflammation through regulation of expression of arthritis-related genes, including those for IL-6, vascular endothelial growth factor-A, and RANKL, in synovial fibroblasts. More recently, Yao et al. (2009) used T cells from mice deficient in $\mathrm{EP}_{2}$ or $\mathrm{EP}_{4}$ and found that $\mathrm{EP}_{2}$ and $\mathrm{EP}_{4}$ receptors redundantly facilitate IL12-mediated Th1 differentiation and IL-23-mediated Th17 expansion. $\mathrm{EP}_{4}$ also functions in production of IL-23 from activated dendritic cells. Development of immune inflammation in experimental allergic encephalomyelitis is consistently and significantly suppressed by treatment of mice with $\mathrm{EP}_{4}$-selective antagonist or in $\mathrm{EP}_{4}$-deficient mice. On the contrary to these proinflammatory actions in immune inflammation, $\mathrm{EP}_{4}$ can exert anti-inflammatory action. Kabashima et al. (2002) found that $\mathrm{EP}_{4}$-deficient mice developed severe colitis in response to treatment with $3 \%$ dextran sodium sulfate, a dose that can be tolerated in wild-type animals. Again, this phenotype was mimicked by administration of an $\mathrm{EP}_{4}$-selective antagonist to wild-type mice. $\mathrm{EP}_{4}$ deficiency was shown to result in impairment of mucosal barrier function that was associated with epithelial loss, crypt damage, and accumulation of neutrophils and $\mathrm{CD}^{+} \mathrm{T}$ cells in the colon. DNA microarray analysis revealed increased expression of genes associated with immune responses and reduced expression of genes associated with mucosal repair and remodeling in the colon of $\mathrm{EP}_{4}$-deficient mice. Given the elevated level of $\mathrm{PGE}_{2}$ and high expression of COX-2 in the brains of patients with Alzheimer's disease, Hoshino et al. (2007) examined the role of $\mathrm{PGE}_{2}$ in processing $\beta$-amyloid precursor protein in cultured cells and found that $\mathrm{PGE}_{2}$ stimulated production of amyloid- $\beta$ peptide by activating $\gamma$-secretase and that this action occurs via $\mathrm{EP}_{2}$ and $\mathrm{EP}_{4}$. They then cross-mated an Alzheimer's disease model of APP-23 transgenic mice with either $\mathrm{EP}_{2}(-/-)$ mice and $\mathrm{EP}_{4}(-/-)$ mice and found that the levels of $A \beta$ peptides were significantly lower in the presence of either deletion. Besides these actions in complex inflammatory diseases, $\mathrm{EP}_{4}$ is also implicated in a simple form of acute inflammation. Kabashima et al. (2007) subjected the ear of mice deficient in each of the EP subtypes to ultraviolet B irradiation and examined the extent of skin inflammation. They found that ear swelling caused by UV irradiation was significantly suppressed with reduced inflammatory cell infiltration and blood flow in $\mathrm{EP}_{2}(-/-)$ and $\mathrm{EP}_{4}(-/-)$ mice compared with wild-type mice and that the effect of $\mathrm{EP}_{4}$ deficiency was mimicked by administration of an $\mathrm{EP}_{4}$ antagonist to wild-type mice. They further found that blockade of $\mathrm{EP}_{2}$ and $\mathrm{EP}_{4}$ is additive, suggesting that they function redundantly.

Epidemiological as well as experimental studies have implicated COX isoforms and PGs in familiar adenomatous polyposis and development of colon cancer. To gain 
an insight into the receptor involved in this process, Watanabe et al., (1999) and Mutoh et al. (2002) examined azoxymethane-induced formation of aberrant cryptic foci in mice deficient in each prostanoid receptor. They found that the formation of such foci was suppressed in both EP1(-/-) and EP4(-/-) mice but not in those deficient in other receptor types or subtypes. In both instances, the number of foci was reduced to 50 to $60 \%$ of that apparent in wild-type mice.

Although systemically null $\mathrm{EP}_{4}$-deficient mice have contributed to our understanding of physiological functions of this receptor, their mixed genetic background has often limited their use. To conquer this weakness, Schneider et al. (2004) generated conditional $\mathrm{EP}_{4}{ }^{\text {flox/flox }}$ mice in which deletion of the $\mathrm{EP}_{4}$ gene can be achieved by expression of Cre recombinase. Using endothelial cells derived from these mice and rendered $\mathrm{EP}_{4}$-null by transfection with adenovirus harboring Cre, Rao et al. (2007) found that $\mathrm{EP}_{4}$ is required for $\mathrm{PGE}_{2}$-mediated migration, in vitro formation of capillary-like structure, cAMP production, and ERK activation. Combining these in vitro findings with in vivo findings that $\mathrm{EP}_{4}$ agonists can induce angiogenesis in sponge implanted into mice, they argued proangiogenic potential for $\mathrm{PGE}_{2}-\mathrm{EP}_{4}$ signaling. Gao et al. (2009) used this $\mathrm{EP}_{4}{ }^{\text {flox/flox }}$ mouse line and generated mice in which one allele of $\mathrm{EP}_{4}$ was globally deleted and the other was targeted in osteoblasts. They found that this line of KO mice developed normal bone and exhibited no change in bone volumes or bone formation, whereas osteoblasts of these mice lost their responsiveness to $\mathrm{PGE}_{2}$ in vitro. They argued that either $\mathrm{EP}_{4}$ signaling may not be required for physiological regulation of bone development and maintenance or the loss of $\mathrm{EP}_{4}$ may be compensated for by other mechanisms. Their findings are not compatible with the previous report by Li et al. (2005), who found osteopenia and impaired fracture healing in aged globally $\mathrm{KO} \mathrm{EP}_{4^{-}}$ deficient mice and that by Akhter et al. (2001), who reported that $\mathrm{EP}_{4}$-deficient mice have small distal femur and vertebral bone volume and exhibit reduced structural and apparent material strength in the femoral shaft and vertebral body. Another study focusing on $\mathrm{EP}_{4}$ in bone using conditional knockout mice examined the action of $\mathrm{EP}_{4}$ in periplastic osteolysis and the identity of the cell type mediating this action. Tsutsumi et al. (2009) implanted polyethylene beads to the periosteal surface of calvaria in $\mathrm{EP}_{1}(-/-)$ mice, $\mathrm{EP}_{2}(-/-)$ mice, and mice with conditionally deleted $\mathrm{EP}_{4}$ in FSP1+ fibroblasts and examined osteolysis. They also prepared fibroblasts and osteoblasts from these mice, stimulated them with titanium beads or $\mathrm{PGE}_{2}$, and assessed production of RANKL. They found that polyethylene-beadinduced osteolysis is impaired only in conditional $\mathrm{EP}_{4}$ $\mathrm{KO}$ mice. It is noteworthy that this conditional knockout exhibited reduced RANKL production only in fibroblasts and not in osteoblasts. On the basis of these findings, the authors suggested that osteolysis associated with total joint replacement is induced by RANKL produced by fibroblasts at the tissue-implant interface. Another example of the use of $\mathrm{EP}_{4}{ }^{\text {flox/flox }}$ mice is generation of mice with loss of $\mathrm{EP}_{4}$ selectively in cardiomyocytes. Qian et al. (2008) generated this line of mice by crossing $\mathrm{EP}_{4}{ }^{\text {flox/flox }}$ with mice carrying Cre recombinase driven by $\alpha$-myosin heavy chain promoter. This line of cardiacspecific $\mathrm{EP}_{4} \mathrm{KO}$ mice does not show any abnormality but exhibits less hypertrophy and less fibrosis with attenuated STS3 activation in a model of myocardial infarction induced by left anterior descending coronary artery ligation. They do, however, show reduced ejection function. A later study by the same group (Harding et al., 2010) revealed that male mice of this line of conditional $\mathrm{EP}_{4}$ knockouts spontaneously develop dilated cardiomyopathy at 23 to 33 weeks of age. Therefore, the cardiospecific $\mathrm{EP}_{4}$-null mice exhibit a cardiac phenotype different from that of systemic $\mathrm{EP}_{4}$-null mice.

d. Agonists and antagonists. Selective $\mathrm{EP}_{4}$ agonists typically contain a 16-phenyl group, the importance of which to vasodilator activity was recognized early (Johnson et al., 1980). Within the ONO series, a 3,7-dithia substitution pattern in the $\alpha$-chain was found to dramatically favor $\mathrm{EP}_{4} / \mathrm{EP}_{3}$ selectivity (Maruyama et al., 2002a). Addition of large groups (e.g., $m$-phenyl) to the 16-phenyl ring resulted in retention of $\mathrm{EP}_{4}$ binding affinity but reduction of functional potency, perhaps because of loss of efficacy (intrinsic activity) (Maruyama et al., 2002b). ONO-AE1-329, with a $m$-methoxymethyl substituent, emerged as a highly selective $\mathrm{EP}_{4}$ full agonist (Cao et al., 2002). Prostanoids with a 8-aza-9-oxo functionality were originally identified as potent ligands for the rat kidney prostaglandin $\mathrm{E}$ receptor (Smith et al., 1977). In the Merck series of selective $\mathrm{EP}_{4}$ agonists, this ring system is combined with a 16-phenyl group, and an acidic 5-tetrazole ring replaces the carboxylate, thereby preventing $\beta$-oxidation (Billot et al., 2003; Young et al., 2004); L-902688 (Fig. 4) seems to be the preferred molecule. CP-734432 (Fig. 4), the active metabolite of 5-(3-(2-(3hydroxy-4-(3-(trifluoromethyl)phenyl)butyl)-5-oxopyrrolidin1-yl)propyl)thiophene-2-carboxylate (PF-04475270), has an $\mathrm{EC}_{50}$ value of $1 \mathrm{nM}$ in a human $\mathrm{rc}-\mathrm{EP}_{4}$ assay (Prasanna et al., 2009). However, 8-aza-9-oxo prostanoids, with a 15,16didehydro-15-methyl structure, switch from $\mathrm{EP}_{4}$ to $\mathrm{EP}_{2}$ agonist selectivity (Brugger et al., 2008). Finally, compound 12 (Fig. 4) in Blouin et al. (2010), which moves away from the classic prostanoid template, is an $\mathrm{EP}_{4}$ full agonist.

The first $\mathrm{EP}_{4}$ antagonist reported was 7-(5-)((1,1biphenyl)-4-yl)methoxy)-2-(4-morpholinyl)-3-oxocyclopentyl)-4-heptanoic acid (AH 23848) (Coleman et al., 1994a), and it played a pivotal role in the early pharmacological definition of the $\mathrm{EP}_{4}$ receptor. $\mathrm{AH} 23848$ was the prototype and has been overtaken by more selective and much more potent compounds, some of which contain a diaryl-acylsulfonamide as a key component of the scaffold: L-161982 (Fig. 5) bears structural resemblance to GW 627368 and BGC-20-1531 (Fig. 5) (Jones et al., 
2009). Other $\mathrm{EP}_{4}$ antagonists are carboxylic acids, such as MK-2894 (Blouin et al., 2010) (Fig. 5). Finally, ER819762 (Fig. 5) is an $\mathrm{EP}_{4}$ antagonist containing a novel spiro ring system (Chen et al., 2010).

e. Therapeutics. The most prominent utility for $\mathrm{EP}_{4}$ antagonists is for treating inflammatory diseases, related hyperalgesia, and allodynia (Jones et al., 2009). In common with most prostanoid receptors, $\mathrm{EP}_{4}$ receptors have been implicated in carcinogenesis, and $\mathrm{EP}_{4}$ antagonists are shown to be effective in animal models (Table 8). It is noteworthy that Yanni et al., (2009) have presented data showing that $\mathrm{EP}_{4}$ receptor antagonists may be useful for treating neovascular eye disease.

The potential uses of $\mathrm{EP}_{4}$ agonist are many and diverse (Table 9). There is enormous diversity: hearing loss (Hori et al., 2009), nephritis (Nagamatsu et al., 2006) glaucoma (Woodward et al., 2009), and myocardial infarction (Xiao et al., 2004). This wide array of $\mathrm{EP}_{4}$ agonist and antagonist activities portends a potential catalog of unwanted side effects, especially associated with oral systemic administration. Beneficial and deleterious side effects may occur in the same target tissue in response to $\mathrm{EP}_{4}$ receptor agonist administration. Although $\mathrm{EP}_{4}$ receptors are cardioprotective in ischemiareperfusion injury (Xiao et al., 2004), they are likely to exacerbate hypertrophy that occurs as an adaptive response to cardiovascular disease (Mendez and LaPointe, 2005; Frias et al., 2007). $\mathrm{EP}_{4}$ receptors stimulate the $\mathrm{BNP}$ promoter and could result in an antifibrotic action in the heart (Qian et al., 2006), which may be compensatory. It seems difficult to assess the pros and cons of treating a patient with heart failure with an $\mathrm{EP}_{4}$ agonist. Added to all this, $\mathrm{EP}_{4}$ agonists are likely to lower blood pressure in humans, because they are well known as potent vasodilators and may also contribute to atherosclerotic plaque destabilization (Cipollone et al., 2005).

Potential cardiovascular and other safety risks are likely to be avoided where local drug delivery is feasible. An $\mathrm{EP}_{4}$ agonist may be useful in treating colitis (Nitta et al., 2002; Jiang et al., 2007), and a compound could perhaps be designed so that systemic absorption is limited. Set against this, $\mathrm{EP}_{4}$ receptors are implicated in colon carcinogenesis (Mutoh et al., 2002; Chell et al., 2006; Doherty et al., 2009), a highly undesirable side effect. In glaucoma therapy, where local topical drug administration is routinely employed, the highly efficacious ocular hypotensive effects produced by $\mathrm{EP}_{4}$ ago- nists are accompanied by ocular surface hyperemia and corneal neovascularization (Aguirre et al., 2009; Prasanna et al., 2009; Woodward et al., 2009). Although some redness of the eyes is not harmful, the patients do not necessarily want it. Perhaps the most therapeutically and commercially successful utility may be for the recently reported utility of $\mathrm{EP}_{4}$ agonists for sensorineural hearing loss (Hori et al., 2009). This would involve local therapy, probably an implant.

\section{FP Receptors}

1. Second Messenger Signaling. The prostanoid FP receptor is predominantly $\mathrm{G}_{\mathrm{q}}$-coupled, with activation of the classic pathway. Thus, after $\operatorname{PLC} \beta$ activation, there is PI turnover, with resultant diacylglycerol-mediated PKC activation and a $\mathrm{Ca}^{2+}$ transient signal in response to inositol trisphosphate formation (Nakao et al., 1993; Abramovitz et al., 1994; Ito et al., 1994; Sugimoto et al., 1994a; Woodward and Lawrence, 1994; Carrasco et al., 1996). Downstream of $G_{q}$, other protein kinases are activated and receptors transregulated. Elevated $\left[\mathrm{Ca}^{2+}\right]_{\mathrm{i}}$ results in calmodulin-mediated myosin light-chain kinase activation (Ansari et al., 2003). PKC activates the Raf/MEK/MAP kinase signaling pathway (Chen et al., 1998; Bos et al., 2004; Husain et al., 2005; Xu et al., 2008). In addition to activating MAP kinases, a PKC/ $\mathrm{Ca}^{2+}$-calcineurin-nuclear factor of activated $\mathrm{T}$ cells pathway has been implicated in $\mathrm{PGF}_{2 \alpha}$-mediated cell growth (Horsley and Pavlath, 2003; Sales et al., 2009). FP receptors may also activate MAP kinases via PLCmediated phosphorylation of the epidermal growth factor (EGF) receptor (Sales et al., 2004).

The repertoire of FP receptor G protein coupling extends beyond $\mathrm{G}_{\mathrm{q}}$. It also includes activation of Rho via $\mathrm{G}_{12} / \mathrm{G}_{13}$ (Pierce et al., 1999). The FP receptor is also reported to couple to $G_{i}$ (Melien et al., 1998; Hébert et al., 2005), providing an alternative route to the Raf/ MEK/MAP kinase pathway (Bos et al., 2004). The early response gene $C y r 61$ is also up-regulated by FP receptor stimulation (Liang et al., 2003) by a pathway sequentially involving Ras/Raf signaling and Tcf transcription independent of MEK/ERK (Xu et al., 2009).

2. Distribution and Biological Functions. FP receptors have a wide distribution and subserve many important functions. The importance of these functions is reflected by the fact that, among the prostanoid receptors, the FP receptor has been the most successful therapeutic target. It has prominent functions in reproduction.

TABLE 8

Potential therapeutic application of $\mathrm{EP}_{4}$ antagonists (inflammation/pain is reviewed in Jones et al., 2009)

\begin{tabular}{|c|c|c|c|c|c|c|}
\hline Agonist & Route & Dose & Species & Experimental Model & Indication & Reference \\
\hline L 161982 & $\begin{array}{l}\text { Intravitreal } \\
\text { injection }\end{array}$ & $0.01-1 \mu \mathrm{M}$ & Rat & $\begin{array}{l}\mathrm{O}_{2} \text {-retinopathy laser induced } \\
\text { choroidal neovascularization }\end{array}$ & $\begin{array}{l}\text { Age-related macular } \\
\text { degeneration }\end{array}$ & Yanni et al., 2009 \\
\hline ONO-AE2-227 & In diet & $100-400 \mathrm{ppm}$ & Mouse & $\begin{array}{l}\text { Azoxymethane induced aberrant } \\
\text { crypt foci; min mouse }\end{array}$ & Colon cancer & Mutoh et al., 2002 \\
\hline ONO-AE3-208 & Oral & $10 \mathrm{mg} / \mathrm{kg}$ & Mouse & Injection of M26 cells & Colon cancer & Yang et al., 2006 \\
\hline
\end{tabular}


TABLE 9

Potential therapeutic application of $\mathrm{EP}_{4}$ agonists

\begin{tabular}{|c|c|c|c|c|c|c|}
\hline Agonist & Route & Dose & Species & Experimental Model & Indication & Reference \\
\hline $\begin{array}{l}\text { 3,7-Dithia } \mathrm{PGE}_{1} \text { and } \\
\text { its isopropyl ester }\end{array}$ & Topical & $0.01-0.1 \%$ & Monkey & $\begin{array}{c}\text { "Glaucomatous" monkey } \\
\text { intraocular pressure }\end{array}$ & Glaucoma & Woodward et al., 2009 \\
\hline $4819-\mathrm{CD}$ & Subcutaneous & $0.3 \mathrm{mg} / \mathrm{kg}$ & Mouse & $\begin{array}{l}\text { Ischemia-reperfusion } \\
\text { injury }\end{array}$ & Myocardial infarction & Xiao et al., 2004 \\
\hline AEI-734 & & & & & Colitis & Kabashima et al., 2002 \\
\hline $\begin{array}{l}\text { AGN } 205203 \text { and its } \\
\text { methyl ester }\end{array}$ & Subcutaneous & $3 \mathrm{mg} / \mathrm{kg}$ & Mouse & $\begin{array}{l}\text { Dextran sodium sulfate } \\
\text { colitis }\end{array}$ & Colitis & Jiang et al., 2007 \\
\hline CP-734432 & Subcutaneous & $0.3-3 \mathrm{mg} / \mathrm{kg}$ & Rat & Ovariectomized rats & Osteoporosis & Ke et al., 2006 \\
\hline ONO-4819 & Subcutaneous & $10,30 \mu \mathrm{g} / \mathrm{kg}$ & Rat & $\begin{array}{l}\text { Femoral drill-hole } \\
\text { injury }\end{array}$ & $\begin{array}{l}\text { Bone fracture healing, } \\
\text { osteoporosis }\end{array}$ & Tanaka et al., 2004 \\
\hline ONO-4819 & Subcutaneous & $3,30 \mu \mathrm{g} / \mathrm{kg}$ & Rat & $\begin{array}{l}\text { Mechanical loading, to } \\
\text { tibia }\end{array}$ & Bone fracture, osteoporosis & Hagino et al., 2005 \\
\hline ONO-4819 & Intravenous & $100 \mathrm{ng} / \mathrm{kg}$ & Rat & Ovariectomized rat & Osteoporosis & Yoshida et al., 2002 \\
\hline ONO-AE1-329 & Intracolonic & $25,100 \mu \mathrm{g} / \mathrm{kg}$ & Rat & $\begin{array}{l}\text { Dextran sodium sulfate } \\
\text { colitis }\end{array}$ & Colitis & Nitta et al., 2002 \\
\hline ONO-AE1-329 & Intravenous & $1-10 \mu \mathrm{g} / \mathrm{kg}$ & Rat & $\begin{array}{l}\text { Indomethacin-induced } \\
\text { lesions }\end{array}$ & $\begin{array}{l}\text { NSAID-induced } \\
\text { gastrointestinal lesions }\end{array}$ & Kunikata et al., 2002 \\
\hline ONO-AE1-329 & Intraperitoneal & $0.1 \mathrm{mg} / \mathrm{kg}$ & Rat & $\begin{array}{l}\text { Indomethacin-induced } \\
\text { lesions }\end{array}$ & $\begin{array}{l}\text { NSAID-induced } \\
\text { gastrointestinal lesions }\end{array}$ & Hatazawa et al., 2006 \\
\hline ONO-AE1-329 & Subcutaneous & $0.1-1000 \mathrm{ng} / \mathrm{g}$ & Rat & $\begin{array}{l}\text { Neonatal ductus } \\
\quad \text { arteriosus dilatation }\end{array}$ & Premature birth & Momma et al., 2005 \\
\hline ONO-AE1-329 & $\begin{array}{l}\text { Local gelatin } \\
\text { plug }\end{array}$ & $1 \mathrm{mg} / \mathrm{ml}$ & $\begin{array}{l}\text { Guinea } \\
\text { pig }\end{array}$ & $\begin{array}{l}\text { Auditory brain stem } \\
\text { response }\end{array}$ & Sensorineural hearing loss & Hori et al., 2009 \\
\hline ONO-AE1-329 & Subcutaneous & $\begin{array}{l}0.05,0.5 \mathrm{mg} / \mathrm{kg} \\
55 \mu \mathrm{g} \text { daily } \\
\text { infusion per } \\
\text { animal }\end{array}$ & Mouse & $\begin{array}{l}\mathrm{N} 2 \text { receptor knockout } \\
\text { mice }\end{array}$ & $\begin{array}{l}\text { Nephrogenic diabetes } \\
\text { insipidus }\end{array}$ & Li et al., 2009 \\
\hline ONO-AE1-329 & Subcutaneous & $100 \mu \mathrm{g} / \mathrm{kg}$ & Mouse & $\begin{array}{l}\text { Anti-glomerular } \\
\text { basement membrane } \\
\text { antibody nephritis }\end{array}$ & Glomerulonephritis & Nagamatsu et al., 2006 \\
\hline ONO-AE1-329 & Intravenous & $1-10 \mu \mathrm{g} / \mathrm{kg}$ & Rat & Endotoxin & Septicemia & Sakamoto et al., 2004 \\
\hline PF-04475270 & Topical & $\begin{array}{l}0.002-0.1 \mathrm{mg} / \\
\mathrm{ml}\end{array}$ & Dog & $\begin{array}{l}\text { Normal dog intraocular } \\
\text { pressure }\end{array}$ & Glaucoma & Prasanna et al., 2009 \\
\hline
\end{tabular}

The FP receptor is highly expressed in ovarian tissue, with mRNA expressed exclusively in the corpus luteum (Sugimoto et al., 1994a). Although the FP receptor is central to corporal luteal regression and regulating the estrous cycle in farm animals (Coleman et al., 1994b), this does not seem to be the case in mice, because mice lacking FP receptors exhibited an unchanged estrous cycle and were fertile (Sugimoto et al., 1997). FP receptors are also present in the human corpus luteum (Narko et al., 1997; Väänänen et al., 1998), but their function is uncertain and certainly not dramatic. In mice lacking the FP receptor, parturition was abolished (Sugimoto et al., 1997). This has stimulated interest in the use of the FP antagonists for preventing preterm labor.

Functional expression of FP receptor in the myometrium mediating contraction has long been known (Senior et al., 1992; Carrasco et al., 1996). FP receptors are also expressed in the human endometrium, where $\mathrm{PGF}_{2 \alpha}$ is biosynthesized in endometrial epithelial cells and causes cell proliferation (Asselin et al., 1997; Milne and Jabbour, 2003). FP receptor expression has been reported in human deciduae, and this has been suggested to contribute to parturition (Makino et al., 2007). By far the most prominent implication of FP receptors in disease is in uterine cancer, specifically endometrial adenocarcinomas. The role of the FP receptors in the progression of endometrial adenocarcinoma includes potentiation of angiogenesis by EGF receptor transactivation and induction of VEGF mRNA expression (Sales et al.,
2005), alteration of adhesion, morphology, and migration (Sales et al., 2008, 2009).

In the $\mathrm{CNS}, \mathrm{PGF}_{2 \alpha}$ given intracisternally alleviates kainic acid-induced seizures potentiated by COX-2 inhibitors, suggesting that $\mathrm{PGF}_{2 \alpha}$ behaves as an endogenous anticonvulsant (Kim et al., 2008). In contrast, FP receptors are claimed to significantly contribute to brain damage associated with focal brain ischemia (Saleem et al., 2009a). The colocalization of the FP receptor and PGF synthase 1 in the spinal cord suggest a role in pain, notably because of intense FP immunostaining in spinal laminae I and II of the dorsal horn (Suzuki-Yamamoto et al., 2009). Indeed, FP receptors mediate $\alpha \mathrm{B}$-methylene ATP-evoked allodynia (Kunori et al., 2009). These data are supported by the finding that spinal intrathecal administration of $\mathrm{PGF}_{2 \alpha}$ produces allodynia by activating FP receptors (Muratani et al., 2003).

Renal expression of FP receptors is high (Sugimoto et al., 1994a; Saito et al., 2003). It is most abundant in the distal convoluted tubule and aquaporin-2-positive cortical collecting ducts (Saito et al., 2003; Hébert et al., 2005). This distribution is consistent with known FPmediated effects on water and solute transport in these segments of the kidney. Renal FP receptors seem to signal via a pertussin toxin-sensitive mechanism (Hébert et al., 2005).

The FP receptor is widely distributed in the human eye (Schlötzer-Schrehardt et al., 2002). Despite high FP receptor expression in the corneal epithelium, ciliary 
epithelium, and iridial stroma and smooth muscle (Schlötzer-Schrehardt et al., 2002), FP agonists are functionally important only in those cells involved in aqueous humor outflow. Thus, ciliary smooth muscle and trabecular meshwork cells are targeted by FP-based therapeutics, without a litany of unwanted side effects. The success of FP agonists, in the form of ester prodrugs, as first-line antiglaucoma agents originated from the vision and determination of Bito (2001). The mechanism by which FP agonists lower intraocular pressure is now entirely understood. FP agonists increase aqueous humor outflow from the anterior segment of the eye, predominantly through the uveoscleral pathway (Stjernschantz et al., 2001; Toris et al., 2005). Effects on trabecular outflow have also been noted in human subjects (Ziai et al., 1993; Toris et al., 2007) and anterior segment preparations (Bahler et al., 2008). Uveoscleral outflow occurs via a widening of the interstitial spacing between ciliary muscle fiber bundles and a controlled remodeling that creates organized drainage channels (Richter et al., 2003). Consistent with a controlled remodeling process, tissue inhibitor of metalloproteinases regulation (Anthony et al., 2002), altered matrix metallopeptidase transcription, and translation products occur (Ocklind, 1998; Sagara et al., 1999; Gaton et al., 2001; Weinreb and Lindsey, 2002). Morphological changes are not restricted to the anterior portion of the ciliary body; they also occur in the trabecular meshwork (Richter et al., 2003). It is not surprising, therefore, that tissue inhibitor of metalloproteinases and matrix metallopeptidase regulation occurs in trabecular meshwork cells (Oh et al., 2006). Gene regulation associated with FP receptor stimulation, however, is not identical in ciliary muscle and trabecular meshwork with respect to genes that would influence aqueous humor dynamics (Zhao et al., 2003). More specifically related to tissue remodeling, Cyr61, connective tissue growth factor, epidermal growth factor receptor-1, and HIF-1 $\alpha$ are upregulated by FP receptor stimulation in ciliary muscle cells (Liang et al., 2003; Hutchinson et al., 2010). The full repertoire of genes that regulate ciliary body remodeling and increased uveoscleral outflow remains to be elucidated.

The side effects associated with FP receptor antiglaucoma therapy are also well understood. The major side effect of latanoprost is iridial hyperpigmentation. This results from a benign eumelanogenic effect, with larger and more mature melanosomes present in iridial melanocytes (Stjernschantz, 2001). Mechanistic investigation demonstrated that latanoprost does not directly stimulate melanocytes but rather activates FP receptors on neighboring fibroblasts (Smith-Thomas et al., 2004). This is quite different from dermal melanocytes, where FP receptor activation directly produces dendricity and increases tyrosinase activity (Scott et al., 2005). FP receptor-induced ocular surface hyperemia involves NOmediated, endothelium-dependent vasodilation (Chen et al., 1995). Latanoprost also produces hypertrichosis of the eyelashes (Johnstone and Albert, 2002).

FP receptor stimulation has long been known to stimulate fibroblast proliferation. FP receptor activation in normal rat kidney fibroblasts adds a new dimension. Here FP receptor stimulation caused membrane depolarization of transformed normal rat kidney cells (Almirza et al., 2008). A positive feedback loop involving FP receptors and COX-2 up-regulation was proposed (Almirza et al., 2008). A most interesting recent report shows that FP receptors facilitate bleomycin-induced pulmonary fibrosis (Oga et al., 2009). FP, via a Rho kinase signaling pathway, produces fibrosis independent of TGF $\beta$, which, until now, has been considered the dominant profibrotic mediator.

Rat ventricular cardiomyocytes undergo hypertrophic growth in response to FP receptor stimulation (Adams et al., 1996; Lai et al., 1996; Pönicke et al., 2000). PGF $_{2 \alpha}$ has also been claimed to mediate inflammatory tachycardia (Takayama et al., 2005). Studies on the contractility of the intact heart have revealed that FP receptors produce a negative inotropic effect, which could contribute to cardiac dysfunction (Jovanović et al., 2006). $\mathrm{PGF}_{2 \alpha}$ seems to have pathophysiological consequences for the cardiovascular system, because it also elevates blood pressure and promotes atherosclerosis (Yu et al., 2009b). PGF ${ }_{2 \alpha}$ also increases skeletal muscle cell growth (Horsley and Pavlath, 2003).

Most prostanoid receptors have been implicated in cancer, and FP is no exception. $\mathrm{PGF}_{2 \alpha}$ stimulates the motility and invasiveness of colorectal tumor cells, with potency equal to that of $\mathrm{PGE}_{2}$ (Qualtrough et al., 2007). $\mathrm{PGF}_{2 \alpha}$-mediated COX-2 up-regulation has been suggested to potentiate tumorigenesis with respect to endometrial adenocarcinoma (Jabbour et al., 2005). Numerous early studies on $\mathrm{PGF}_{2 \alpha}$ have shown a mitogenic effect on fibroblast cell lines, and this was often suggested as contributory to tumor growth (De Asua et al., 1975).

Studies on murine osteoclast development suggest that $\mathrm{PGF}_{2 \alpha}$ would inhibit bone resorption (Kamon et al., 2008). FP receptor expression has been detected in primary human osteoblasts (Sarrazin et al., 2001), and in UMR-106 cells, functional FP receptors were identified (Yamaguchi et al., 1988). Finally, FP receptor activation potently inhibits adipose cell differentiation according to studies on adipocyte precursors obtained from murine inguinal fat pads (Serrero and Lepak, 1997).

3. Gene Deletion Studies. Sugimoto et al. (1997) generated a line of FP-deficient mice by replacing the second exon of the FP gene with $\beta$-galactosidase- and neomycinresistance genes, and this line has been used in all studies below. $\mathrm{PGF}_{2 \alpha}$ is known as a luteolytic substance in animals. Initial analysis of this line of mice revealed that $\mathrm{FP}(-/-)$ female mice did not show any abnormality in the luteal cycle but exhibited parturition failure (Sugimoto et al., 1997). The failure was apparently due to the 
lack of labor, and these mice did not exhibit prepartum decline in the plasma progesterone level. Ovariectomy $24 \mathrm{~h}$ before the expected term decreased the progesterone level and induced normal parturition in $\mathrm{FP}(-/-)$ dams. Given the high expression of FP in the corpora lutea that produces progesterone and the luteolytic action of $\mathrm{PGF}_{2 \alpha}$, these findings suggest that the $\mathrm{PGF}_{2 \alpha}-\mathrm{FP}$ pathway triggers parturition by inducing luteolysis in the ovary.

Several PGF analogs are used as antiglaucoma drugs. $\mathrm{FP}(-/-)$ mice were used to examine the identity of the receptor mediating IOP-lowering activity of these PG analogs. These mice were resistant not only to FP agonists such as latanoprost and travoprost but also to bimatoprost and unoprostone, PG analogs suggested not to bind to FP with high affinity, although these compounds lowered IOP to a comparable extent in wild-type C57BL/6 mice (Crowston et al., 2004, 2005; Ota et al., 2005). These results indicate that FP mediates IOPlowering action of all of these PG analogs at least in mice. Whereas FP mediates potent reduction of IOP in response to exogenously applied $\mathrm{FP}$ agonists, $\mathrm{FP}(-/-)$ mice showed no abnormality in diurnal variation of IOP compared with wild-type mice, indicating that the $\mathrm{PGF}_{2 \alpha}-\mathrm{FP}$ pathway is not involved in physiological regulation of IOP (Crowston et al., 2007). Furthermore, administration of ONO-AE1-259 (Fig. 4), an $\mathrm{EP}_{2}$ agonist, and ONO-AE1-329 (Fig. 4), an $\mathrm{EP}_{4}$ agonist, induced reduction of IOP equally well in wild-type and $\mathrm{FP}(-/-)$ mice, indicating the presence of a PG-dependent pathway different from that of FP in lowering IOP (Saeki et al., 2009).

Systemic inflammation induces many adaptive symptoms, one being tachycardia. Takayama et al. (2005) used mice deficient in individual prostanoid receptors and examined their involvement in this inflammatory tachycardia. They first found that $\mathrm{PGF}_{2 \alpha}$ and a TP agonist, I-BOP, could elevate the heart rate of mice through FP and TP, respectively, and that this action was exerted locally in the atria. They then showed that tachycardia induced by systemic administration of lipopolysaccharide was abrogated partially in either $\mathrm{TP}(-/-)$ or $\mathrm{FP}(-/-)$ mice and completely in mice deficient in both FP and TP receptors. Inflammatory tachycardia was believed to result from increased sympathetic discharge, and the results by Takayama et al. (2005) have changed this traditional view. The function of $\mathrm{PGF}_{2 \alpha}$ in the cardiovascular system was further elucidated by the use of $\mathrm{FP}(-/-)$ mice (Yu et al., 2009b). Systolic blood pressure in $\mathrm{FP}(-/-)$ mice was significantly lower than that of wild-type mice, with significantly lower plasma concentrations of renin and angiotensin-1 in $\mathrm{FP}(-/-)$ mice. To explore the basis of this phenotype, they examined FP receptor localization in the kidney and found that FP is expressed in the preglomerular artery (Yu et al., 2009b), in addition to previously identified localization in distal tubules and col- lecting ducts (Saito et al., 2003); stimulation of FP increased renin mRNA expression in isolated JG cells, though it did not stimulate renin release. In addition, FP is expressed in the medial smooth muscle layer of resistant arterioles, and infusion of $\mathrm{PGF}_{2 \alpha}$ elevated blood pressure in wild-type but not in $\mathrm{FP}(-/-)$ mice. Yu et al. (2009b) further cross-mated FP(-/-) with mice deficient in the LDL receptor, and reported that atherosclerosis was significantly attenuated on the $\mathrm{FP}(-/-)$ background. Although macrophage infiltration and inflammatory cytokine expression in atherosclerotic plaques were diminished in $\mathrm{FP}(-/-)$ mice, it remained unclear how FP is involved in atherogenesis. Saleem et al. (2009a) subjected $\mathrm{FP}(-/-)$ mice to transient brain ischemia-reperfusion by reversible ligation of the middle cerebral artery. They found that the loss of FP does not alter physiological parameters such as cerebral blood flow, $\mathrm{PaO}_{2}, \mathrm{PaCO}_{2}$, mean arterial blood pressure, and body temperature before, during, or after brain ischemia. Nonetheless, $\mathrm{FP}(-/-)$ mice exhibited significantly less neurological deficit and smaller infarct volume than wild-type mice. To examine whether this difference is due to excitoneurotoxicity associated with transient ischemia-reperfusion, they then injected NMDA into the striatum unilaterally and found that $\mathrm{FP}(-/-)$ mice exhibited significantly smaller infarct volumes than wildtype mice again under these conditions, indicating that FP somehow regulates the excitation of glutamatergic neurons after ischemic injury. Consistent with these findings, they showed that administration of latanoprost widened the infarction and worsened the deficit. Finally, Oga et al. (2009) subjected mice deficient in individual prostanoid receptor to bleomycin-induced pulmonary fibrosis and found significant suppression of fibrosis in $\mathrm{FP}(-/-)$ mice. They found that pulmonary inflammation comparable with that in wild-type mice was induced by bleomycin, but subsequent fibrosis was suppressed in FP(-/-) mice. Microarray analysis revealed significant attenuation of the induction of genes associated with fibrosis, such as various isoforms of collagen and other matrix proteins in $\mathrm{FP}(-/-)$ mouse lung, and FP stimulation in cultured lung fibroblasts facilitated cell proliferation and collagen production in vitro. It is noteworthy that the loss of FP receptors did not affect expression and activation of TGF- $\beta$, a critical cytokine in fibrosis, and $\mathrm{PGF}_{2 \alpha}$ and TGF- $\beta$ contributed additively to fibrosis. On the basis of these results, the authors suggested that $\mathrm{PGF}_{2 \alpha}$ functions as a mediator of fibrosis independent of TGF- $\beta$, and FP may be a drug target for idiopathic pulmonary fibrosis in humans.

4. Agonists and Antagonists. Early studies established the high FP selectivity of the (racemic) 16- $m$ trifluoromethyl-phenoxy analog of PGF $_{2 \alpha}$ (ICI-81008, fluprostenol; Dukes et al., 1974), particularly its minimal TP agonism (Welburn and Jones, 1978). Fluprostenol continues to be the FP agonist of choice and is commercially available as the more active (+)-enantio- 
mer. The 16- $m$-chlorophenoxy analog of $\mathrm{PGF}_{2 \alpha}$ (cloprostenol) is the most potent $\mathrm{FP}$ agonist reported but retains considerable $\mathrm{EP}_{3}$ agonism; change to a 3-hydroxy-tetrahydofuran ring with shift of the 5,6-cis bond to the 4 position as in AL-12180 (Fig. 6) improved the selectivity ratio (Sharif et al., 2006). 13,14-Dihydro-17-phenyl $\mathrm{PGF}_{2 \alpha}$ (latanoprost-free acid) also has high FP selectivity, showing 22-fold less $\mathrm{EP}_{1}$ agonism than 17-phenyl $\mathrm{PGF}_{2 \alpha}$ (Ungrin et al., 2001). Introduction of a 2-indanyl group into the $\omega$-chain has resulted in claims for FP antagonism (Griffin et al., 1999; Sharif et al., 2000; AL-3138, AL-8810), although partial agonism seems to be a better description (Woodward et al., 2007). Structures are given in Fig. 6. Woodward et al. (2000) reported that although the $\mathrm{C} 1$-alcohol and C1-methyl ether analogs of $\mathrm{PGF}_{2 \alpha}$ were very weak agonists at FPreceptors of cat and human, they showed high potency on the cat lung parenchyma preparation, an action that could not be ascribed to other known prostanoid receptors. Subsequent studies have suggested the existence of a new receptor type, the prostamide $\mathrm{F}$ receptor, which recognizes carboxyl, alcohol, ethanolamide, and alkylamide moieties at $\mathrm{C} 1, \mathrm{PGF}_{2 \alpha}$-ethanolamide (Fig. 6) being a potential natural ligand (Woodward et al., 2008). In cat iris sphincter digests, 17-phenyl $\mathrm{PGF}_{2 \alpha}$-ethylamide (bimatoprost) activated one set of cells and 17-phenyl $\mathrm{PGF}_{2 \alpha}$ and $\mathrm{PGF}_{2 \alpha}$, another (Spada et al., 2005).

$\mathrm{C}-1$ amine and amide derivatives of $\mathrm{PGF}_{2 \alpha}$ show weak FP antagonism in some systems, but their utility is severely limited (see Jones et al., 2009). 15-Indanyl- $\omega$ pentanor PGF analogs, such as AL-3138 and AL-8810 (Fig. 7), represent improvements (Griffin et al., 1999; Sharif et al., 2000). However, AL-8810 has modest affinity for $\mathrm{FP}$ receptors $\left(\mathrm{IC}_{50}, 8.7 \mu \mathrm{M}\right.$ in human ciliary muscle cells; Sharif et al., 2006) and blocks TP receptors in mouse uterus (Hutchinson et al., 2003), pig ciliary artery (Vysniauskiene et al., 2006), and human recombinant receptor assays (Krauss and Woodward, unpub- lished). Partial block of epidermal growth factor-induced contraction of guinea pig trachea by AL-8810 (Schaafsma et al., 2005) may be explained by TP antagonism rather than the proposed FP antagonism. Moreover, both PGF analogs often exhibit FP partial agonism (Griffin et al., 1999; Hutchinson et al., 2003), and AL-8810 was even a full agonist in the cat iris preparation, an action not blocked by a prostamide $\mathrm{F}$ antagonist (Woodward et al., 2007). More potent, selective prostamide F antagonists, such as AGN 211334 (Fig. 7) have more recently been reported (Wan et al., 2007; Woodward et al., 2008).

Peptides of the THG series (Chemtob and Peri, 2006; Peri et al., 2009) block PGF $_{2 \alpha}$-induced responses, but this may not necessarily involve competition for the FP receptor. The octapeptide THG-113.31 [Ile-Leu-Gly-His(D-Cit)-Asp-Tyr-Lys] insurmountably blocked $\mathrm{PGF}_{2 \alpha^{-}}$ induced contraction of pig retinal blood vessels, while having minimal effect on contraction to 17-phenyl $\mathrm{PGE}_{2}$, U-46619, phenylephrine, Angiotensin II and endothelin-1. However, the affinity of TGH-131.31 for the human recombinant FP receptor was poor $(\sim 13 \%$ at 10 $\mu \mathrm{M})$. Moreover, TGH-131.31 $(10 \mu \mathrm{M})$ weakly antagonized contraction of sheep myometrium induced by $\mathrm{PGF}_{2 \alpha}$ and had no effect on $\mathrm{PGF}_{2 \alpha}$-induced contraction of human pregnant myometrium but inhibited spontaneous and oxytocin-induced contractions at much lower concentrations (Friel et al., 2005). At 10 to $50 \mu \mathrm{M}$, TGH113.31 enhanced $\mathrm{BK}_{\mathrm{Ca}}$ channel opening in human uterine myocytes, which was reversed by iberiotoxin (Doheny et al., 2007). Simpler peptides (e.g., TGH-113.824; Fig. 7) also block $\mathrm{PGF}_{2 \alpha}$-induced contraction (Peri et al., 2009).

5. Therapeutics. By far the greatest therapeutic success of prostanoid pharmacology is the use of FP receptor agonist prodrugs for the treatment of glaucoma. Latanoprost was the first in its therapeutic class (Bito, 2001; Stjernschantz, 2001). This was followed by the

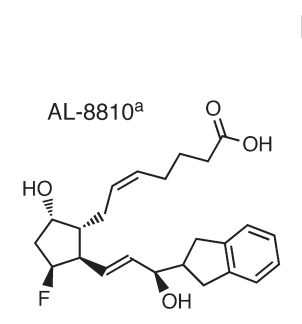

FP agonists
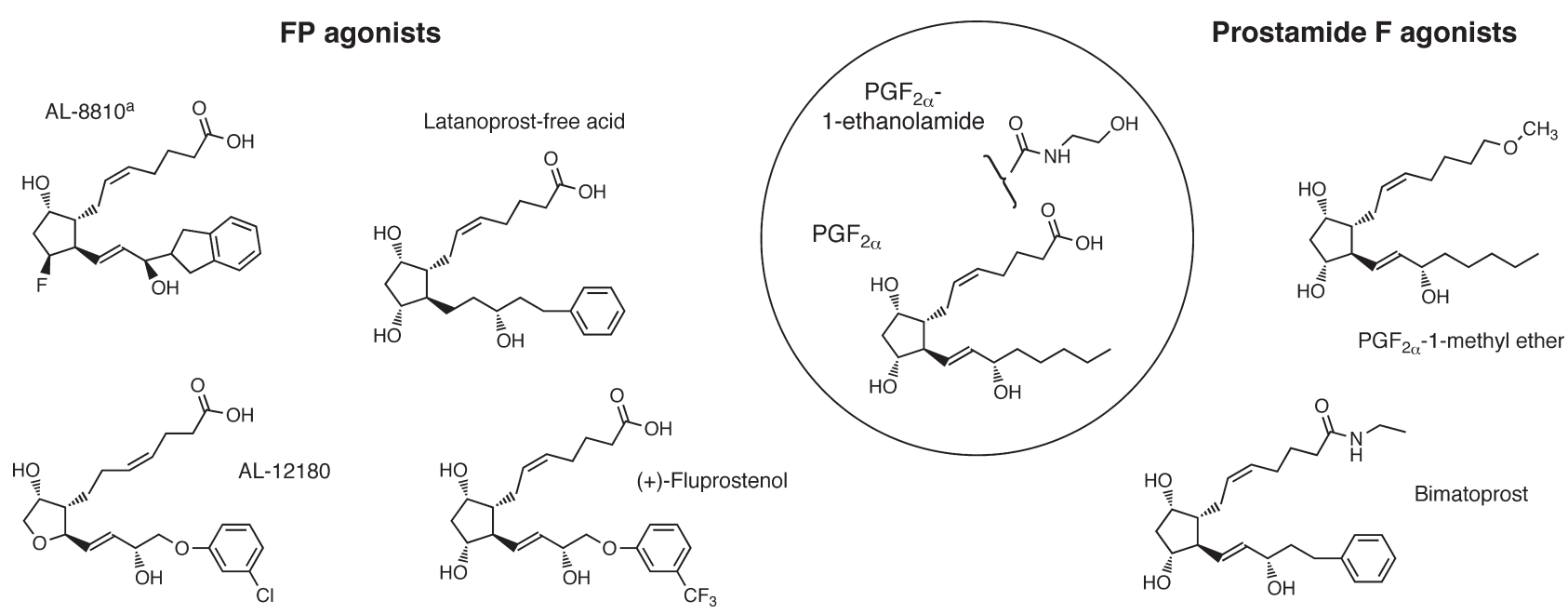

FIG. 6. Structures of agonists for the prostanoid FP receptor and the corresponding prostamide $\mathrm{F}$ receptor. PGF ${ }_{2 \alpha}$ potently activates both receptors, whereas its $\mathrm{C} 1$-ethanolamide activates only the prostamide $\mathrm{F}$ receptor. ${ }^{\mathrm{a}}$, partial agonist. 


\section{FP antagonists}

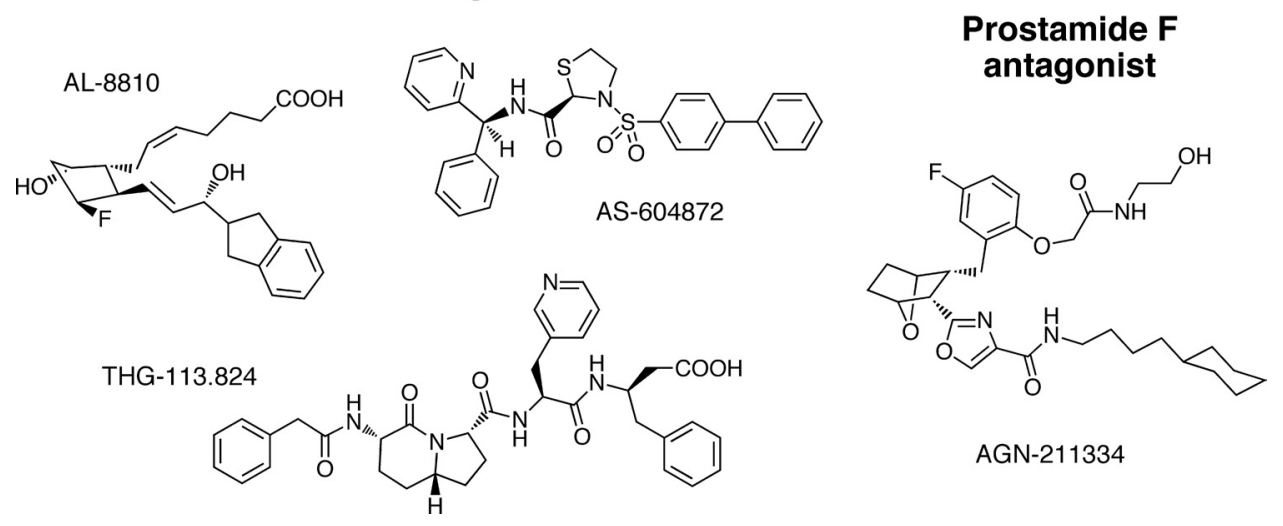

FIG. 7. Structures of FP antagonists and the prostamide antagonist (AGN 211334).

isopropyl ester of fluprostenol, named travoprost (Hellberg et al., 2001) and tafluprost (Takagi et al., 2004; Cirillo et al., 2007). Comparisons between these drugs are widely available and are not reviewed here.

An alternative therapeutic use for FP agonists emerged from studies on glaucoma: hair growth. Latanoprost and related compounds cause eyelash growth (Johnstone and Albert, 2002). Studies in the stumptailed macaque model of androgen-induced scalp alopecia showed latanoprost to be effective (Uno et al., 2002). This opportunity does not seem to have been commercially realized. The reasons for this are obscure. For this use, the domain of pharmacocosmetics (Woodward et al., 2008) is accessed. In the domain of cosmetics, unlike treating pain, inflammation, cancer, etc., the options are not restricted to drug intervention and surgery. Cosmetic aspirations can be instantly gratified by colorants, surgery, or implantation/replacement. This may have discouraged development of latanoprost for male pattern baldness. However, acceptance of cosmetic intervention and instant results is widely accepted by women but not by men. A gradual improvement in natural scalp hair is likely to be more socially acceptable to men because it would avoid derision from friends and colleagues. Regrowth of natural scalp hair would take a long time, because anaphase extends over years. In addition, like any other disease, early intervention is essential. These may have been major discouraging factors. On the other hand, the psychological impact of male pattern baldness cannot be underestimated, and balding men are typically highly motivated and inclined toward subtle and natural intervention. FP receptor expression has been detected in the human dermal papillae (Colombe et al., 2008), which supports a favorable outcome for human clinical investigation. Fat reduction/antiobesity is a further potential pharmacocosmetic/medical use of FP agonists, but this is still at the adipocyte precursor stage essentially (Serrero and Lepak, 1997).

With the exception of osteoporosis, the effects of FP receptor activation are pathophysiological, and antagonist therapy would be the requirement. The major focus has been the prevention of preterm labor. These antagonists include the peptide THG-113.31 and derivates (Olson, 2005; Chemtob and Peri, 2006; Jones et al., 2009). The non-PG structure FP antagonist AS-604872 (Fig. 7) (Cirillo et al, 2007) seems to have a better commercial prospect as an orally bioavailable small molecule. It showed no agonism in a human FP receptorinositol phosphate assay $\left(\mathrm{IC}_{50}, 47 \mathrm{nM}\right.$ for $\left.\mathrm{PGF}_{2 \alpha}\right)$. In vivo, AS-604872 (1-30 mg/kg i.v.) inhibited $\mathrm{PGF}_{2 \alpha^{-}}$ induced uterine contraction in the nonpregnant rat: inhibition of oxytocin-induced contractions was slight (Chollet et al., 2007). These apart, no serious attention seems to have been given to FP antagonist design. This may change in light of reports implicating FP receptors in cardiovascular disease (Yu et al., 2009b) and fibrosis (Oga et al., 2009).

\section{IP Receptors}

1. Second Messenger Signaling. The IP receptor has long been known to be both $\mathrm{G}_{\mathrm{s}}$ - and $\mathrm{G}_{\mathrm{q}}$-coupled, resulting in increased cAMP formation, $\mathrm{PI}$ turnover, and $\mathrm{Ca}^{2+}$ signaling (Coleman et al., 1994; Namba et al., 1994; Narumiya et al., 1999). IP receptor activation of the cAMP-PKA pathway seems to be exclusively responsible for some prostacyclin-mediated events (Nasrallah et al., 2001; Ritchie et al., 2004; Kamio et al., 2007; Muja et al., 2007). Solution structure studies indicate that the first and third intracellular loops of the IP receptor are in contact with $\mathrm{C}$-terminal residues of $\mathrm{G} \alpha_{\mathrm{s}}$ to initiate cAMP signaling (Zhang et al., 2006a; Zhang et al., 2006b). $\mathrm{N}$-glycosylation is involved in both adenylyl cyclase and inositol phosphate formation (Zhang et al., 2001).

IP receptors are not unique in activating dual or multiple signaling but seem to be among the best studied. $G_{i}$ and $G_{q}$ coupling to IP receptors has been reported to be dependent on $G_{s}$ coupling as a prerequisite, the key event being phosphorylation of $\mathrm{Ser}^{357}$ of the IP receptor by PKA (Lawler et al., 2001). This mechanism is consistent with investigations invoking both cAMP and cAMPindependent mechanisms in many instances. These include MaxiK channel activation (Yamaki et al., 2001) 
and STAT3 phosphorylation (Lo et al., 2008), in addition to straightforward analysis of dose-dependent effects on cAMP formation (Accomazzo et al., 2002). PKAmediated switching from $G_{s}$ to $G_{q}$ and/or $G_{i}$ coupling, however, does not seem to be a universal phenomenon (Chow et al., 2003). G-protein coupling seems to be cellspecific. No evidence for $G_{i}$ coupling could be found in NG108-15 and SK-N-SH cells and in Chinese hamster ovary or human embryonic kidney 293 cells expressing recombinant IP receptors (Chow et al., 2003). In marked contrast, $\mathrm{G}_{\mathrm{i}}$-coupled IP receptors were found exclusively in human erythroleukemia HEL cells with respect to STAT1 and STAT3 phosphorylation (Lo et al., 2006) and rat medullary thick ascending limb cells (Hébert et al., 1998). Finally, but beyond the intended scope of this review, there are reports claiming that prostacyclin interacts with peroxisome proliferator-activated receptors to exert a portion of its activities (Lim and Dey, 2002; Ali et al., 2006).

2. Distribution and Biological Functions. The role of prostacyclin in cardiovascular homeostasis is well publicized. Thus, COX-2 inhibitors may present a cardiovascular hazard by depressing prostacyclin production by vascular endothelial cells without a concomitant reduction in platelet COX-1-derived $\mathrm{TxA}_{2}$. This results in a potentially deleterious effect on thrombosis and blood pressure and accelerated atherogenesis (Cheng et al., 2002; Dogné et al., 2005; Wang et al., 2005). Of particular relevance is that COX-2 expression and $\mathrm{PGI}_{2}$ production in human coronary arterial endothelial cells may be increased by proinflammatory stimuli (Tan et al., 2007). These findings imply that local $\mathrm{PGI}_{2}$ produced in the coronary vasculature may directly counteract the vasoconstriction and platelet aggregation production by $\mathrm{TxA}_{2}$ during episodic vascular insult. The cardioprotective action of prostacyclin has been proposed to extend to preventing atherosclerosis, intimal hyperplasia, and restenosis (Fetalvero et al., 2007).

Vascular smooth muscle cells are not terminally differentiated and can proliferate and further differentiate. IP receptors inhibit vascular smooth muscle cell proliferation (Lin et al., 2008) by inhibiting $\mathrm{G}_{1}$-to-S-phase progression (Fetalvero et al., 2007) and inducing apoptosis (Li et al., 2004). Prostacyclin also induces genes characteristic of a contractile phenotype and inhibits vascular smooth muscle cell migration (Blindt et al., 2002; Fetalvero et al., 2007). Vascular endothelial cells are, in addition to vascular smooth muscle cells, an important source of prostacyclin, which is induced by laminar shear stress (Di Francesco et al., 2009). Beyond prostacyclin effects on vascular smooth muscle and endothelial cells, the inhibitory effects on platelet aggregation and its vasodilator properties should not be understated. IP receptor stimulation increases retinal, choroidal (Mori et al., 2007), and coronary blood flow (Gwóźdź et al., 2007). In ApoE-deficient mice, IP receptors conferred protection against the initiation and pro- gression of atherogenesis by limiting platelet activation and leukocyte-vascular endothelial cell interaction (Kobayashi et al., 2004). Mice lacking IP receptors showed augmented cardiac hypertrophy in response to pressure overload (Hara et al., 2005) and increased myocardial infarct size after ischemia-reperfusion injury (Xiao et al., 2001), suggesting a cytoprotective effect on cardiomyocytes independent of platelet and neutrophil inhibition. IP (-/-) mice also develop salt-sensitive hypertension and cardiac fibrosis, in addition to cardiac hypertrophy (Francois et al., 2005).

Continuing the subject of systemic hypertension, decreased susceptibility to renovascular hypertension was reported in mice lacking IP receptors (Fujino et al., 2004). Prostacyclin, therefore, indirectly produces hypertension in the two-kidney, one-clip hypertension model: the mechanism seems to involve increased renin mRNA expression and production according to studies on cicaprost in juxtaglomerular cells (Fujino et al., 2004). In addition to juxtaglomerular cells, IP message is also present in the renal cortex, outer and inner medulla, and inner medullary collecting duct, according to reverse transcription-polymerase chain reaction (Nasrallah et al., 2001). Localization studies using in situ hybridization detected IP receptor mRNA in the tubules of the inner and outer medulla, the vasculature, and in the arteries, glomeruli, and tubules of the cortex (Nasrallah et al., 2001). Renal IP receptor distribution in murine species does not necessarily correlate with that in humans (Nasrallah and Hébert, 2005). No obvious pathological renal condition is apparent in $\operatorname{IP}(-/-)$ mice. Prostacyclin, however, may also be involved in renal hypertrophy, fibrosis, and apoptosis (Nasrallah and Hébert, 2005).

The prostanoid IP receptor plays a prominent role in edema formation, hyperalgesia, and pain (Bley et al., 1998; Hata and Breyer, 2004). Nociceptive responses in the acetic acid writhing model are virtually abolished in mice lacking IP receptors (Murata et al., 1997). In models of rheumatoid arthritis, such as carrageenin-induced paw edema and collagen antibody-induced arthritis (Murata et al., 1997; Ueno et al., 2000; Honda et al., 2006; Pulichino et al., 2006), a pronounced reduction is observed in IP (-/-) mice. Likewise, pleurisy induced by carrageenin (Yuhki et al., 2004) and zymosan (Yuhki et al., 2008) is attenuated in mice lacking IP receptors. Results obtained with IP antagonists in rat models of pain and inflammation (Bley et al., 2006; Pulichino et al., 2006) are consistent with results obtained in the IP $(-/-)$ mice studies. The involvement of IP receptors in pain and hyperalgesia are supported by IP expression studies (Bley et al., 1998; Doi et al., 2002) and functional studies on neurons conducting at $\mathrm{C}$ velocity (Smith et al., 1998). Studies on dorsal root ganglia support an IP receptor role in sensitization of sensory neurons (Smith et al., 1998; Rowlands et al., 2001; Nakae et al., 2005). 
IP receptor expression in the thymus and spleen was reported more than a decade ago (Narumiya et al., 1999). Since then, some insight into IP receptor involvement in lymphocyte function has emerged. IP receptors tend to suppress Th2-mediated responses (Hata and Breyer, 2004). Thus, IP receptor stimulation inhibits Th2 cytokine (IL-4, IL-10, IL-13) and Th1 cytokine (IFN $\gamma$ ) production (Zhou et al., 2007). Plasmacytoid dendritic cells, which are believed critical for controlling adaptive immunity, also express functional IP receptors, which suppressed Toll-like receptor-mediated $\mathrm{TNF} \alpha$ and INF $\gamma$ production and enhanced IL-10 production (Hung et al., 2009). IP signaling has been claimed to prevent recruitment of Th2 cells into airways in an asthma model (Jaffar et al., 2007), but the use of highly unstable $\mathrm{PGI}_{2}$ confounds interpretation. Nevertheless, because allergic airway and cutaneous inflammation involving Th2 cells in the same species is augmented in IP receptor-deficient mice (Takahashi et al., 2002), it seems correct that IP receptors inhibit Th2 cell function. Further support is provided by a study that implicates prostacyclin in the anti-inflammatory activity of 1-methylnicotinamide in experimental contact hypersensitivity (Bryniarski et al., 2008). Finally, in the macrophage cell line Raw 264.7, IP activation via LPS-induced prostacyclin production generates VEGF (Park et al., 2007). Because this pathway involved Akt signaling, it is relevant to angiogenesis and cancer. IP receptors up-regulate angiogenic genes in the human endometrium (Smith et al., 2006).

IP receptors have been functionally characterized in the human myometrium (Senior et al., 1992). Prostacyclin synthase and IP receptor expression are increased during the menstrual phase (Battersby et al., 2004), indicating a possible role in normal and/or dysfunctional menstruation. Perhaps a better case could be made for $\mathrm{PGI}_{2}$ participation in pregnancy and labor by upregulating contractile proteins and connexin 43 that could prime the myometrium for parturition (Fetalvero et al., 2008; Taggart et al., 2008). IP receptors have also been implicated in preimplantation embryo development (Huang et al., 2007a) and may contribute to embryo transport by decreasing the amplitude of oviductal contractility (Arbab et al., 2002).

Prostacyclin receptor deletion has been shown to aggravate hippocampal neuronal loss caused by ischemia (Wei et al., 2008) and cortical cell loss after traumatic brain injury (Lundblad et al., 2008). These experiments indirectly suggest that IP receptors could promote neuronal survival. The ulcerogenic response to ischemiareperfusion was increased in severity in $\operatorname{IP}(-/-)$ mice, suggesting that IP receptors play a crucial role in gastric mucosal defense in vascular injury (Kotani et al., 2006). IP involvement in adaptive cytoprotection produced by mild irritants seems controversial (Boku et al., 2001; Takeuchi et al., 2001b).
3. Gene Deletion Studies. Two lines of IP-deficient mice were generated independently (Murata et al., 1997; Cheng et al., 2002). A study on IP(-/-) mice (Kotani et al., 2006) showed that, although these animals develop and age normally, they manifest an increased thrombotic tendency in the presence of endothelial damage. These findings confirmed the long-held view of $\mathrm{PGI}_{2}$ as an endogenous antithrombotic agent and suggest that this antithrombotic system is activated in response to vascular injury. Indeed, Cheng et al. (2002) examined the interplay between IP and TP signaling in response to vascular injury by subjecting $\operatorname{IP}(-/-)$ mice and $\mathrm{TP}(-/-)$ mice to vascular injury by a balloon catheter. They found that IP deficiency increased, whereas TP deficiency decreased, injury-induced vascular proliferation and platelet activation. They further showed that the augmented response apparent in $\operatorname{IP}(-/-)$ mice was abolished by ablation of TP. Such augmented intimal hyperplasia was also seen in IP(-/-) mice subjected to common carotid artery ligation and transplant arteriosclerosis (Rudic et al., 2005). Furthermore, Xiao et al. (2001) subjected IP-deficient mice to cardiac ischemia-reperfusion injury and found that $\operatorname{IP}(-/-)$ mice exhibited a significantly larger size of myocardial infarct than wildtype mice, whereas $\mathrm{TP}(-/-)$ showed no difference, suggesting that receptor $\mathrm{PGI}_{2}$-IP signaling exerts a protective action during these conditions. As for the role of IP in chronic vascular disease, Kobayashi et al. (2004) examined progression of atherosclerosis in ApoE $(-/-) /$ IP $(-/-)$ double-knockout mice and found that atherosclerosis was accelerated in these mutant animals, despite the fact that they manifested similar plasma cholesterol and triglyceride concentrations. The lumen of the innominate artery was almost completely occluded in 45-week-old ApoE(-/-)/IP(-/-) mice. Mice deficient in IP do not show abnormalities of blood pressure under basal conditions. There are two reports suggesting the involvement of IP in development of hypertension under some conditions. Fujino et al. (2004) examined the contribution of prostanoids to the development of this condition by subjecting mice deficient in either IP or each of the four EP subtypes to a twokidney, one-clip model of renovascular hypertension. They found that hypertension in this model was ameliorated in IP-deficient mice but not in any of the EPdeficient animals. Consistent with these observations, plasma renin activity, the abundance of renin mRNA in the kidney, and the plasma concentration of aldosterone were all substantially reduced in the IP knockout animals compared with those in the wild type. Given that IP is expressed in the afferent glomerular arterioles, that expression of the renin gene is expanded to these arterioles in response to reduced perfusion of the kidney, and that $\mathrm{PGI}_{2}$ induces renin release from cultured juxtaglomerular cells in vitro, these researchers suggested that $\mathrm{PGI}_{2}$-IP signaling directly stimulates renin release. Alternatively, such signaling may regulate the perfusion 
pressure of the juxtaglomerular apparatus locally and induce renin release indirectly. Francois et al. (2005) found that IP-deficient mice fed on a high-salt $(6 \% \mathrm{NaCl})$ diet developed a significantly larger increase in blood pressure than wild-type mice without alteration in renin and aldosterone levels, and concomitantly developed cardiac hypertrophy and fibrosis. Their finding on saltinduced hypertension in $\operatorname{IP}(-/-)$ is consistent with that of Watanabe et al. (2005), who found that with a highsalt diet, systolic blood pressure in female $\operatorname{IP}(-/-)$ mice gradually increased, whereas that in the $\mathrm{IP}(+/+)$, $\mathrm{TP}(-/-)$, or $\mathrm{TP}(+/+)$ mice remained unchanged. On the other hand, Hara et al. (2005) examined cardiac hypertrophy and fibrosis in response to pressure overload. They subjected mice deficient in each prostanoid receptor to banding of the transverse aorta and found augmented hypertrophy and fibrosis only in $\operatorname{IP}(-/-)$ mice. Thus, a protective role of IP signaling in cardiac hypertrophy and fibrosis is found in mice on two different backgrounds and different models.

There are several studies using $\mathrm{IP}(-/-)$ mice to determine the role of IP in pain sensation and inflammatory swelling. Murata et al. (1997) found that in IPdeficient mice, the acetic acid writhing response was reduced to a level similar to that observed in wild-type mice treated with the COX inhibitor indomethacin. The capsaicin receptor TRPV1 is a nociceptor for heat and $\mathrm{pH}$. Moriyama et al. (2005) found that thermal hyperalgesia in response to $\mathrm{PGI}_{2}$ is absent not only in $\mathrm{IP}(-/-)$ mice but also in TRV1 $(-/-)$ mice. They further found that capsaicin-activated current in dorsal root ganglia neurons was augmented by $\mathrm{PGI}_{2}$ as well as an IP agonist, and this augmentation was absent in neurons from $\mathrm{IP}(-/-)$ mice. It is noteworthy that the TRV1 augmentation is seen at a rather high concentration of $\mathrm{PGI}_{2}$, $1000 \mathrm{nM}$. As for inflammatory swelling, Murata et al. (1997) subjected IP-deficient mice to the carrageenininduced paw swelling model and found that inflammatory swelling was reduced by $\sim 50 \%$ in these animals, an effect similar in magnitude to that achieved by treatment of wild-type mice with nonsteroidal anti-inflammatory drugs. Yuhki et al. (2004) showed that IP, as well as $\mathrm{EP}_{2}$ and $\mathrm{EP}_{3}$ receptors, mediate exudate formation in carrageenin-induced mouse pleurisy, suggesting that $\mathrm{PGE}_{2}$ and $\mathrm{PGI}_{2}$ elicit inflammatory responses in a context-dependent manner (i.e., one dependent on the type of stimulus and site in the body) and that their contribution may also change during the course of inflammation. The inflammatory action of IP is not limited to inflammatory swelling exerted through regulation of the peripheral circulation in acute inflammation. Honda et al. (2006) examined the role of prostanoids by backcrossing mice deficient in each prostanoid receptor on a DBA background and subjecting them to collageninduced arthritis. They found that, whereas the incidence of arthritis was unaffected, the extent and progression of this condition were markedly suppressed in
IP-deficient mice as well as in $\mathrm{EP}_{2}$-deficient mice treated with an $\mathrm{EP}_{4}$ antagonist. These findings thus indicated that the $\mathrm{PGI}_{2}$-IP signaling and $\mathrm{PGE}_{2}$ signaling at $\mathrm{EP}_{2}$ and $\mathrm{EP}_{4}$ receptors mediate joint inflammation in this model. Further analysis revealed that both $\mathrm{PGI}_{2}$ and $\mathrm{PGE}_{2}$ pathways regulate expression of arthritis-related genes, including those for IL-6, vascular endothelial growth factor-A, and RANKL, in synovial fibroblasts and thereby contribute to arthritic inflammation, bone destruction, and pannus formation. On the other hand, Pulichino et al. (2006) evoked arthritis by administering collagen antibodies to their IP(-/-) mice on a C57BL/6 background and found that arthritis was almost completely suppressed in this line of mice. They further used a newly developed IP antagonist [ $N$-[4-(imidazolidin-2ylideneamino)-benzyl]-4-methoxy-benzamide], administered it into heterozygous $\operatorname{IP}(+/-)$ mice before the antibody injection or after the onset of arthritis, and examined its prophylactic or therapeutic effects. They found that although the prophylactic administration of the IP antagonist exerted suppression of arthritis in $\mathrm{IP}(+/-)$ mice comparable with that in $\mathrm{IP}(-/-)$ mice, it showed no effects when administered after the disease onset. These results suggest that the $\mathrm{PGI}_{2}$-IP signaling functions as part of the process where self-reactive antibodies trigger the disease. However, whether this is the same mechanism as that identified by (Honda et al., 2006) remains unknown. In addition, starting with the finding that COX-2-deficient mice show exaggerated fibrotic response in bleomycin-induced pulmonary fibrosis, Lovgren et al. (2006) subjected mice deficient in either $\mathrm{EP}_{2}, \mathrm{EP}_{4}$, or IP to this model. They found that only IP-deficient mice exhibited significantly enhanced fibrosis and suggested that COX-2-derived $\mathrm{PGI}_{2}$ exerts a protective action against fibrosis in this model.

$\mathrm{PGI}_{2}$ has been shown to exert a protective effect on the gastric mucosa in response to injurious stimuli. IPdeficient mice have also been used to examine this phenomenon. Boku et al. (2001) and Arai et al. (2003) examined the role of IP in releasing CGRP in the stomach in response to a mild stimulant, $1 \mathrm{M} \mathrm{NaCl}$, or capsaicin, which exerts protection to ethanol. They found that release of CGRP to both stimuli is impaired in $\operatorname{IP}(-/-)$ mice, and coadministration of capsaicin and beraprost enhanced the release, suggesting that endogenous $\mathrm{PGI}_{2}$-IP signaling functions to release CGRP for gastric protection. Furthermore, Terashima et al. (2009) used IP(-/-) mice and showed that iloprost can decrease histamine-stimulated acid secretion in the stomach in an IP-dependent manner. They further found that this IP-mediated decrease is dependent on the somatostatin SST2 receptor. On the basis of these findings, they suggested that $\mathrm{PGI}_{2}$ receptors may mediate somatostatin release in the gastric mucosa, which in turn suppresses acid secretion. Given expression of IP in various types of immune cells, including macrophages and T cells, Takahashi et al. (2002) subjected IP-deficient mice to the 
OVA-induced asthma model and examined its role. They found that $\operatorname{IP}(-/-)$ mice exhibited substantially higher plasma concentrations of antigen-specific and total $\mathrm{IgE}$, indicating that $\mathrm{PGI}_{2}$-IP signaling is somehow involved in sensitization to IgE production. Finally, IP $(-/-)$ mice were used to distinguish some actions of PGI analogs on IP and PPAR $\beta$. Ali et al. (2006) used lung fibroblasts from $\operatorname{IP}(-/-)$ mice and those from $\operatorname{PPAR} \beta(-/-)$ mice, examined inhibitory effects of treprostinil on their proliferation, and found that this activity was lost in PPAR $\beta(-/-)$ cells but not in $\operatorname{IP}(-/-)$ cells.

4. Agonists and Antagonists. The upper row in Fig. 8 shows stabilization of the vinyl ether of $\mathrm{PGI}_{2}$ by carbon replacement (iloprost), electron withdrawal with fluorine (AFP-07), and conjugation with or within aromatic moieties (beraprost, taprostene). In each case, orientation of the $\alpha$-carboxyl terminus away from the $\omega$-chain, which is crucial to potent IP agonism, is maintained; a more detailed treatment may be found in Wise and Jones (1996). A 16-methyl/18.19-triple bond structure has often been favored for the $\omega$ terminus. Iloprost and AFP-07 also show potent $\mathrm{EP}_{1}$ agonism and to a lesser extent $\mathrm{EP}_{3}$ agonism, whereas cicaprost is more selective (Dong et al., 1986; Lawrence et al., 1992; Abramovitz et al., 2000; McCormick et al., 2010) and is the preferred choice for an IP standard agonist. Taprostene is a IP partial agonist (Jones and Chan, 2001, 2005).

EP-157 (Fig. 8) is a $\mathrm{PGH}_{2}$ analog exhibiting both TP antagonism and IP agonism (Armstrong et al., 1986); the diphenylmethyl-heteroatomic group is critical to the latter activity (Jones et al., 1993). The related compounds in Fig. 4 also possess a similar or 1,2-diphenylethyl group and have been referred to as "nonprostanoid prostacyclin mimetics" (Meanwell et al., 1994). BMY-45778 (Fig. 8) is the most potent agent within this subclass (Jones et al., 1997; Seiler et al., 1997). Caution is neces- sary in using these agonists to characterize IP receptors because of their high lipophilicity, partial agonism (octimibate; Merritt et al., 1991a) and ability to inhibit $\mathrm{G}_{\mathrm{q}} / \mathrm{PLC}$-driven effects (Chow et al., 2003). ONO-1301 (Kondo et al., 1995 also inhibits $\mathrm{TxA}_{2}$ synthase because of the presence of a 3-pyridyl group). MRE-269 is obtained by hydrolysis of the methylsulfonamide moiety in the prodrug NS-304 (Kuwano et al., 2007, 2008).

Chemical library screening was the starting point for two classes of selective IP antagonist (Bley et al., 2006): RO-1138452 is a 2-(phenylamino)-imidazoline and RO3244019 is an N-substituted phenylalanine (Fig. 9). In addition to its high affinity for human native (platelet) and recombinant IP receptors $\left(\mathrm{p} K_{\mathrm{i}}, 9.3\right.$ and 8.7, respectively), RO-1138452 also binds to platelet-activating factor $\left(\mathrm{p} K_{\mathrm{i}}, 7.9\right)$ and imidazoline $\left(\mathrm{p} K_{\mathrm{i}}, 8.3\right)$ receptors (Bley et al., 2006). RO-1138452 exhibited surmountable antagonism in functional studies on blood vessel preparations from human, rabbit, and guinea pig, with $\mathrm{p} A_{2}$ values in the range 8.1 to 8.4 (Jones et al., 2006). Higher concentrations of RO-1138452 slightly suppressed the cicaprost maximum response, probably because of the $\mathrm{EP}_{3}$ agonist action of cicaprost. In contrast, insurmountable antagonism was found for RO-1138452 in a chemokine release assay involving human airway epithelial cells (Ayer et al., 2008). RO-1138452 inhibition of IP agonist-induced cAMP response element-dependent transcription was not reversed after a 20 -h "washout" period. It was proposed that allosterism or a state of antagonist hemiequilibrium and may underlie this profile (Ayer et al., 2008).

5. Therapeutics. Prostacyclin infusion has been in medical use for some time for treating pulmonary hypertension (Wise and Jones, 1996; Olschewski et al., 2004; Gryglewski 2008; Mubarak, 2010). The stable prostacyclin analog iloprost provides several practical advantages for intravenous therapy and may be given by
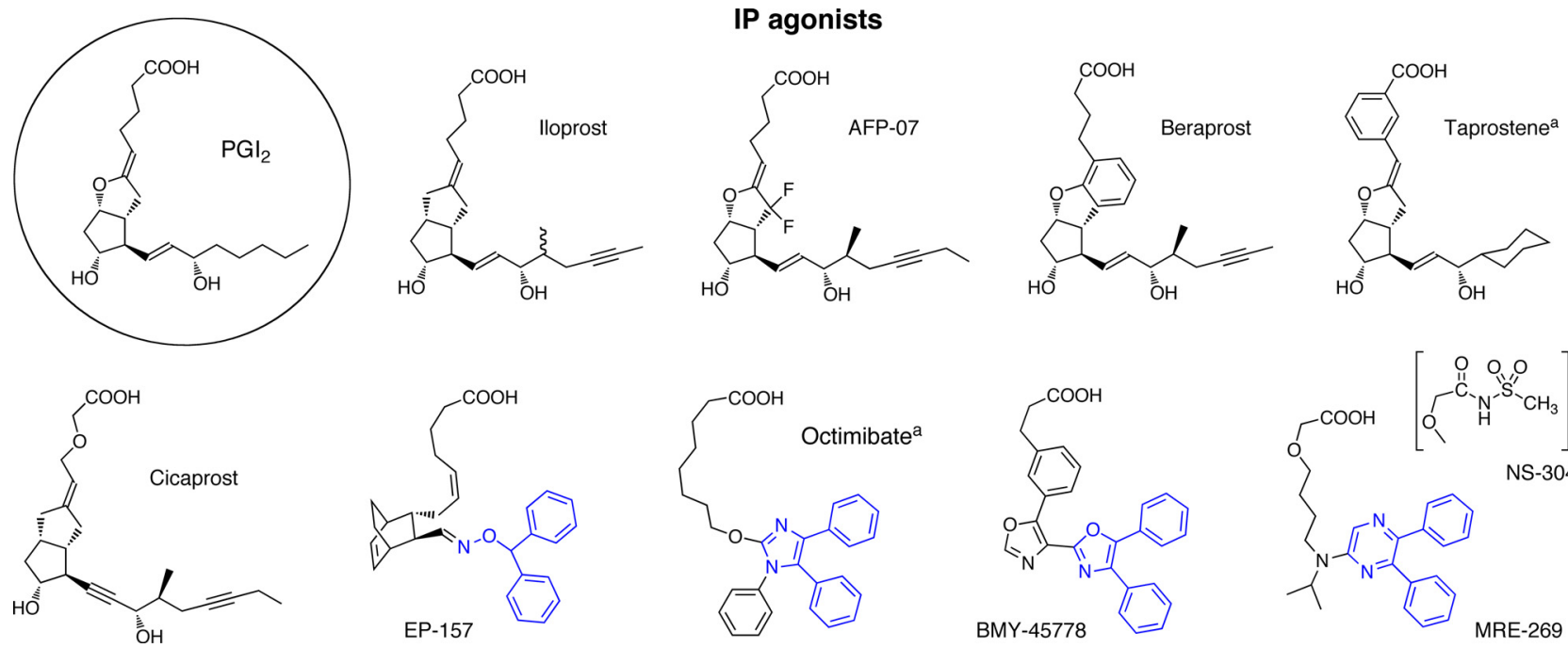

FIG. 8. Structures of agonists for the prostanoid IP receptor. PGI $_{2}$ (prostacyclin), the most active natural agonist, is shown in the circle. The

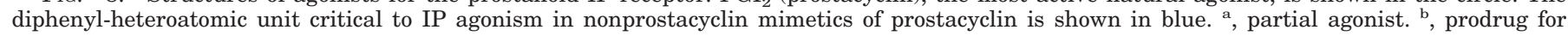
MRE-269. 


\section{IP antagonists}
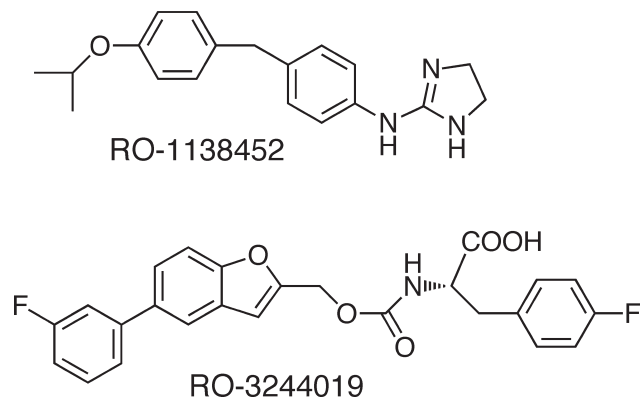

FIG. 9. Structures of IP receptor antagonists.

aerosol (Olschewski et al., 2004; Mubarak, 2010). Treprostinil was developed for subcutaneous administration, and beraprost is an orally active prostacyclin mimetic (Olschewski et al., 2004; Mubarak, 2010). Prostacyclin and its analogs are also useful for treating advanced critical limb ischemia that may occur in Buerger's disease, Raynaud's syndrome, and atherosclerosis (Zardi et al., 2005; Gryglewski, 2008). New IP agonists continue to be developed, the latest being NS-304 (Fig. 8). This is both long-acting and has better selectivity than beraprost or iloprost and represents better future therapy for pulmonary arterial hypertension (Kuwano et al., 2008). Beyond critical care, IP agonists have not enjoyed wide use as medical therapy. The potential for profound decreases in blood pressure and for IP receptor-induced pain and inflammation provide a significant barrier to widespread use. Other limited and certain local therapeutic applications may be possible.

There is also an IP receptor therapeutic focus on designing antagonists. The impetus for this is to provide analgesic/anti-inflammatory agents, with no effect on bleeding time (Bley et al., 2006; Pulichino et al., 2006; Brescia et al., 2007; Zhao et al., 2008; Jones et al., 2009). A potential use for IP antagonists for treating overactive bladder disorders has been advanced. Thus, RO3244019 decreased bladder contraction frequency and increased the micturition threshold in isovolumetric bladder contraction and refill models, respectively (Cefalu et al., 2007), and improved intercontractile interval and voiding volumes as assessed by cystometry (Khera et al., 2007).

\section{E. TP Receptors}

1. Second Messenger Signaling. TP receptors have been shown to couple to $G_{\mathrm{q}}$, thereby initiating the PLC $\beta$ $\rightarrow$ inositol trisphosphate/diacylglycerol $\rightarrow\left[\mathrm{Ca}^{2+}\right]_{\mathrm{i}} / \mathrm{PKC}$ signaling cascade (Hirata et al., 1991; Dorn and Becker, 1993; Kinsella et al., 1997; Offermanns, 2006; Nakahata 2008). Subsequent studies have strongly implicated $\mathrm{G}_{12 / 13^{-}}$ Rho as a major signaling pathway for TP receptors (Huang et al., 2004; Honma et al., 2006; Mir and Le Breton, 2008; Nakahata, 2008; Song et al., 2009; Zhang et al., 2009; Saito et al., 2010). More than any other prostanoid receptor, TP receptors have been subjected to extensive second messenger pathway analyses. Thus, at the molecular level, there is detailed understanding of $\mathrm{TP}$ receptor-G protein interactions obtained by using G protein fusion constructs and guanosine $5^{\prime}-O$-(3-thio) triphosphate binding studies (Hildebrandt, 2006; Zhang et al., 2006, 2009). The $\mathrm{G}$ protein coupling repertoire for TP receptors is beyond extensive; it seems all-encompassing. Other members of the $\mathrm{G}_{\mathrm{q}}$ family are TP receptor-linked: $\mathrm{G}_{11}$ (Kinsella et al., 1997), $\mathrm{G}_{15}$, and $\mathrm{G}_{16}$ (Offermanns and Simon, 1995), activating the $G_{\mathrm{q}}$-mediated signaling cascade. TP receptors may couple to $\mathrm{G}_{\mathrm{s}}$ (Hirata et al., 1996; Walsh et al., 1998; Mir and Le Breton, 2008) and $\mathrm{G}_{\mathrm{i}}$ (Ushikubi et al., 1994; Song et al., 2009). Finally, TP receptors are reported to couple to $G_{h}$ (Vezza et al., 1999).

TP receptor signaling may result in transactivation, for example of ERK 1/2 (Nakahata, 2008) and EGF receptors (Uchiyama et al., 2009). Likewise, TP receptors possess a PKA/protein kinase G phosphorylation site and four PKC phosphorylation sites, providing mechanisms for modulation/desensitization (Huang et al., 2004). The type of $\mathrm{G}$ protein coupling may also alter $\mathrm{TP} \alpha$ conformation, which may influence ligand binding and activation of the receptor (Zhang et al., 2006).

Human TP receptors exist in two isoforms, TP $\alpha$ and TP $\beta$ (Hirata et al., 1991; Raychowdhury et al., 1994). $\mathrm{TP} \beta$ is an alternative mRNA splicing variant with an extended carboxyl terminus. The differences in intracellular termini may influence desensitization, internalization, and G protein coupling (Parent et al., 1999; Reid and Kinsella, 2007; Nakahata, 2008). Thus, the TP $\beta$ isoform may couple to $\mathrm{G}_{\mathrm{i}}$ (Hirata et al., 1996). However, only the TP $\alpha$ isoform is translated in platelets (Habib et al., 1999). It is noteworthy that because TP receptors play a prominent role in mediating the activity of isoprostanes, heterodimerization of $\operatorname{TP} \alpha$ and $\operatorname{TP} \beta$ have been reported to enhance isoprostane signaling (Wilson et al., 2007).

2. Distribution and Biological Functions. The platelet has always been a major focus of TP receptor research. The TP receptor was originally purified from human platelets (Ushikubi et al., 1989). TP receptor activation produces shape change and platelet aggregation, providing a positive feedback event for causing thrombus formation and thrombosis (Offermanns, 2006; Nakahata, 2008). TP receptor-deficient mice exhibit prolonged bleeding times and are unable to form stable thrombi (Thomas et al., 2001). It has been reported that $\mathrm{TxA}_{2}$ promotes soluble CD40 ligand release from platelets (Enomoto et al., 2010) The platelet $\mathrm{TxA}_{2}$-vascular endothelial $\mathrm{PGI}_{2}$ balance is of central importance to the rational for low-dose aspirin therapy and is the basis for COX-2 inhibitor cardiovascular side effects, underscoring the pathophysiological importance of TP receptors. 
Beyond platelets, TP receptors are widely expressed in the cardiovascular system and represent a liability for cardiovascular disease at all levels. In endothelial cells, $\mathrm{TxA}_{2}$ accelerates the expression of adhesion proteins (Ishizuka et al., 1998) and impairs insulin signaling (Song et al., 2009). Increased $\mathrm{TxA}_{2}$ production may contribute to the development of endothelial dysfunction, with resultant vasoconstriction (Gendron and Thorin, 2007; Francois et al., 2008; Denniss and Rush, 2009; Félétou et al., 2009; Graham and Rush, 2009). TxA 2 has also been implicated in atherogenesis (Dogné et al., 2005), and mice that are TP receptor-deficient develop fewer atherosclerotic lesions (Kobayashi et al., 2004). The presence of TP receptors on peripheral blood monocytes (Allan and Halushka, 1994) would contribute to the formation of atherosclerotic plaque. Cardiovascular TP receptor expression even extends to cardiomyocytes, and the $\mathrm{TxA}_{2}$ mimetic U-46619 (Fig. 10) causes arrhythmia (Wacker et al., 2009). TP receptors are also located on afferent sympathetic nerve endings in the heart and may participate in the sympathoexcitatory reflex that occurs during myocardial ischemia (Fu et al., 2008).
Indirect evidence for functional TP receptor expression on peripheral sensory neurons is provided by the pruritogenic activity of U-46619 (Andoh et al., 2007). Introduction of U-46619 into the fourth ventricle rapidly produces emesis (Kan et al., 2008). In relation to the CNS, TP receptors are reported to be expressed on oligodendrocytes (Blackman et al., 1998; Mir and Le Breton, 2008) and human astrocytoma cells (Honma et al., 2006).

In addition to blood vessels, TP receptors are expressed in several smooth muscle types (Coleman et al., 1994b; Nakahata, 2008). Among these, the lung has always featured prominently. The presence of TP receptors on human bronchial smooth muscles was pharmacologically defined in 1989 (Coleman and Sheldrick, 1989), to provide early impetus to TP receptor studies in the bronchopulmonary system. TP receptors played a substantial role in mediating leukotriene-mediated bronchoconstriction (Piper and Samhoun, 1981; Weichman et al., 1982). $\mathrm{TxA}_{2}$ is a potent constrictor of bronchial smooth muscle and has been implicated in both asthma and chronic obstructive pulmonary disease (Ro-

\section{TP agonists}

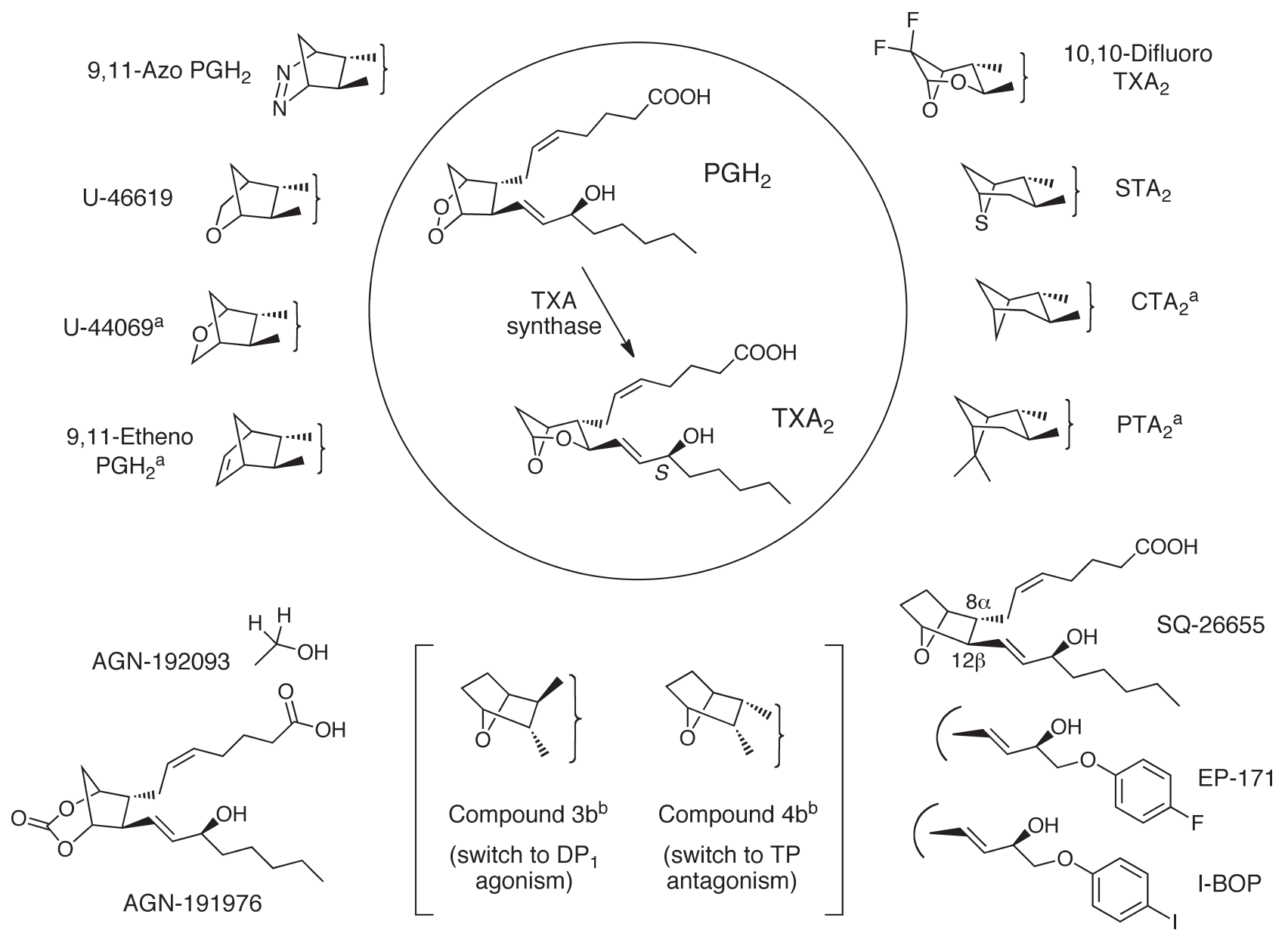

FIG. 10. Structures of agonists for the prostanoid TP receptor. $\mathrm{TxA}_{2}$ and $\mathrm{PGH}_{2}$, the most active natural agonists, are shown in the circle. Only structural alterations relative to $\mathrm{PGH}_{2}$ and $\mathrm{TxA}_{2}$ are illustrated in the figure. The vertical bracket indicates the presence of 2 -series $\alpha$ - and $\omega$-chains. ${ }^{\text {a }}$, partial agonist. 
lin et al., 2006). TP receptors are also uterotonic (Senior et al., 1991) and seem to be particularly important in parturition, because $\mathrm{PGF}_{2 \alpha}$ responsiveness, but not that of $\mathrm{TxA}_{2}$, is lost in the human myometrium during labor (Fischer et al., 2008). Finally, on the smooth muscle cell theme, TP receptors seem capable of differentiating human stem cells to smooth muscle-like cells (Kim et al., 2009).

In common with all prostanoid receptors, TP receptors have been implicated in cancer. U-46619 has been reported to stimulate endothelial cell migration in vivo and angiogenesis and lung metastasis in vivo (Nie et al., 2000). TP receptors also cause proliferation and growth of human lung cancer cells (Li and Tai, 2009; Wei et al., 2010). TP receptors are elevated in prostate cancer and stimulate motility of human prostate cancer cell lines (Nie et al., 2008). It is noteworthy that the TP $\beta$ isoform, but not $\mathrm{TP} \alpha$, promoted cell proliferation and migration and invasion by bladder cancer cells; moreover, TP $\beta$ receptor expression was increased in tissue obtained from bladder cancer patients (Moussa et al., 2008).

Among the additional functions attributed to TP receptors is their role in immune regulation. Naive $\mathrm{T}$ cells obtained from mice expressing TP receptors suppress interaction between dendritic cells and inhibit dendritic cell-dependent $\mathrm{T}$ cell proliferation (Kabashima et al., 2003a). In TP-deficient mice, immune responses to foreign antigenic stimuli were enhanced, a phenomenon reproduced in wild-type mice by TP receptor blockade during the sensitization period (Kabashima et al., 2003a). In contrast, splenocytes obtained from IPdeficient mice exhibited a reduced proliferative response to phytohemagglutinin or anti-CD3 antibody (Thomas et al., 2003). Although survival of transplanted hearts from wild-type mice into TP-deficient mice was not prolonged, there was reduced pathological severity in the allografts (Thomas et al., 2003). A role for $\mathrm{TxA}_{2}$ in inflammatory bowel disease is also a possibility (Rampton and Collins, 1993). The vasoconstrictor activity of TP receptors may also be a factor in renal disease (Michel et al., 2008; Araujo and Welch, 2009). TxA 2 has also been advanced as a key regulator during Trypanosoma cruzi infection and may contribute to mortality and parasitism (Ashton et al., 2007). Finally, thromboxane synthase and TP receptors are expressed in the murine retina and vasoconstriction in the streptozotocin diabetes model was attenuated by TP receptor blockade (Wright et al., 2009a,b).

3. Gene Deletion Studies. Two lines of TP(-/-) mice were generated independently (Thomas et al., 1998; Kabashima et al., 2003a). Both lines of mice show no abnormalities of blood pressure under the basal conditions but do show increased bleeding tendencies and are resistant to cardiovascular shock induced by intravenous infusion of arachidonic acid or the TP agonist U-46619. Given the antagonistic actions of $\mathrm{TxA}_{2}$ and $\mathrm{PGI}_{2}$ on platelets and blood vessels, TP signaling in cardiovascu- lar homeostasis was studied using these lines of mice by comparing their phenotypes with those of $\mathrm{IP}(-/-)$ mice. Cheng et al. (2002) subjected IP(-/-) mice and TP(-/-) mice to vascular injury by a balloon catheter and found that IP deficiency increased injury-induced vascular proliferation and platelet activation, whereas TP deficiency decreased it. They further showed that the augmented response apparent in $\operatorname{IP}(-/-)$ mice was abolished by ablation of TP. These results showed that, once endothelial integrity is disrupted, IP functions protectively, whereas TP aggravates the remodeling. The same group also examined the involvement of TP signaling in vascular remodeling using the external carotid artery ligation model that retains endothelial integrity (Rudic et al., 2005). In this model, treatment of animals with nimesulide augmented the neointimal hyperplastic response of the artery, which was reduced by the loss of TP receptors, suggesting again that $\mathrm{TxA}_{2}-\mathrm{TP}$ signaling facilitates vascular remodeling after injury or stress. During the above ligation model, production of not only $\mathrm{TxA}_{2}$ but 8,12-iso-isoprotane $\mathrm{F}_{2 \alpha}$ was increased. Isoprostanes (iPs) are free radical-catalyzed products of arachidonic acid that reflect lipid peroxidation in vivo. Among them, $\mathrm{iPF}_{2 \alpha}-\mathrm{III}$ and $\mathrm{iPE}_{2}-\mathrm{II}$ can activate platelets and increase vascular tone. The pressor response in vivo and platelet aggregation in vitro induced by these substances were abolished in $\mathrm{TP}(-/-)$ mice, suggesting that actions of these iPs are mediated by TP (Audoly et al., 2000). To test the involvement in chronic vascular diseases, Kobayashi et al. (2004) generated the $\operatorname{ApoE}(-/-) / \mathrm{TP}(-/-)$ double-knockout mice, and examined the effects of TP deficiency on the progression of atherosclerosis. In contrast to acceleration of atherosclerosis in the $\mathrm{ApoE}(-/-) /$ IP $(-/-)$ double-knockout mice, atherosclerosis was delayed in the $\mathrm{ApoE}(-/-) / \mathrm{TP}(-/-)$ mice, despite the fact that they manifested similar plasma cholesterol and triglyceride concentrations. A recent bone marrow transfer experiment (Zhuge et al., 2006) indicated that the effects of TP deficiency attenuating atherogenesis cannot be attributed simply to bone marrow-derived cells such as macrophages. Finally, Francois et al. (2005) found that IP(-/-) mice developed salt-sensitive hypertension, which led to cardiac hypertrophy and severe cardiac fibrosis, and coincidental deletion of TP did not suppress hypertension but ameliorated the hypertrophy and abolished the fibrosis, suggesting that $\mathrm{TxA}_{2}-\mathrm{TP}$ signaling is responsible for hypertensioninduced cardiac complications. These findings have verified that the presumed antagonistic roles of the $\mathrm{PGI}_{2}$-IP signaling and $\mathrm{TxA}_{2}$-TP signaling exists in not only subacute vascular remodeling but also chronic vascular lesions such as atherosclerosis and cardiac fibrosis and may explain the increased incidence of cardiovascular events that has been observed in clinical trials with COX-2 inhibitors. Thus, the loss of TP usually suppresses the progression of pathological vascular conditions. However, a paradoxical example was reported in kidney lesions induced by $N^{\mathrm{G}}$-nitro-L-arginine methyl ester. Francois et al. 
(2008) subjected $\mathrm{TP}(-/-)$ mice to the $N^{\mathrm{G}}$-nitro-L-arginine methyl ester-induced hypertension model and found that the extent of hypertension and resultant cardiac hypertrophy were significantly suppressed in $\mathrm{TP}(-/-)$ mice compared with wild-type mice but that the kidney lesion, including glomerulosclerosis, tubule vacuolization, and chronic intestinal inflammation, was worsened with the loss of TP, suggesting that $\mathrm{TxA}_{2}$-TP signaling exerts protective actions against kidney injury, at least in this model. In addition, as described in the FP section, the inflammatory tachycardia induced by either cytokines or LPS was suppressed in mice deficient in TP or FP and was almost completely abolished in mice deficient in both TP and FP. Because both $\mathrm{PGF}_{2 \alpha}$ and I-BOP, a TP-selective agonist, induced potent positive chronotropic effects on direct application to the nodal area, these results suggest that both $\mathrm{TxA}_{2}$ and $\mathrm{PGF}_{2 \alpha}$ are produced in response to inflammatory stimuli in situ in the heart and act on TP and FP receptors expressed on pacemaker cells to induce tachycardia. In addition, $\mathrm{TxA}_{2}$ - $\mathrm{TP}$ signaling also regulates the microcirculation under pathological conditions. During endotoxemia, TNF- $\alpha$ induces dysfunction of the hepatic microcirculation. Katagiri et al. (2008) found that this TNF- $\alpha$-induced dysfunction, examined as leukocyte adhesion to microvessel endothelial cells, increased the number of nonperfused sinusoids, which was significantly lessened in $\mathrm{TP}(-/-)$ mice, indicating that $\mathrm{TNF}-\alpha$ mobilizes $\mathrm{TxA}_{2}-\mathrm{TP}$ signaling and impairs the microcirculation.

In addition to the cardiovascular actions of TP described above, studies on TP $(-/-)$ mice revealed thus-farunappreciated actions of TP in immunity and inflammation. Kabashima et al. (2003a) noticed lymphadenopathy and splenomegaly in aged $\mathrm{TP}(-/-)$ mice and found that immune response to foreign antigens was enhanced in $\mathrm{TP}(-/-)$ mice. They further found that stimulation of TP receptors enhanced chemokinesis of $\mathrm{T}$ lymphocytes and down-regulated dendritic cell-dependent T-cell proliferation in vitro. These findings led them to suggest that $\mathrm{TxA}_{2}$ - $\mathrm{TP}$ signaling in $\mathrm{T}$ cells regulates the interaction between $\mathrm{T}$ cells and dendritic cells and thereby the extent of the immune response. On the other hand, Thomas et al. (2003) found that T cells deficient in TP receptor exhibited less proliferation in response not only to phytohemagglutinin or anti-CD3 antibody but also in the mixed lymphocyte response (MLR). Reduced proliferation of $\mathrm{TP}(-/-)$ lymphocytes in MLR was mimicked by the MLR of wildtype $\mathrm{T}$ cells treated with a TP antagonist, 7-(3-((2((phenylamino)carbonyl)hydrazino)methyl)-7-oxabicyclo (2.2.1)hept-2-yl)-5-heptenoic acid (SQ-29548) or a thromboxane synthase inhibitor, carboxyhexyl imidazole. These findings are opposite those of Kabashima et al. (2003a), who found that T-cell proliferation in MLR was suppressed by treatment with a TP agonist, I-BOP. Thomas et al. (2003) reported that their TP(-/-) mice exhibited prolonged cardiac allograft survival compared with wild-type mice. We do not know why such a discrepancy arose using different lines of $\mathrm{TP}(-/-)$ mice. In addition to these functions of TP in the peripheral immune system, $\mathrm{TxA}_{2}-\mathrm{TP}$ signaling also seems to regulate the immune system centrally. Ushikubi et al. (1993) found that TP mRNA was markedly expressed in the thymus, particularly in $\mathrm{CD} 4^{-} / \mathrm{CD} 8^{-}$and $\mathrm{CD} 4^{+} / \mathrm{CD} 8^{+}$ immature thymocytes, and that the addition of a TP agonist, stable $\mathrm{TxA}_{2}$ analog, induced apoptosis of $\mathrm{CD}^{+}$/ $\mathrm{CD}^{+}$cells. Rocha et al. (2005) found that administration of LPS into mice markedly increased production of $\mathrm{TxA}_{2}$ and $\mathrm{PGE}_{2}$ in the thymus, and caused apoptotic deletion of $\mathrm{CD}^{+} / \mathrm{CD} 8^{+}$cells there. They then demonstrated that thymocyte apoptosis in response to LPS was significantly attenuated in $\mathrm{TP}(-/-)$ mice, suggesting that thymocyte apoptosis mediated by $\mathrm{TxA}_{2}-\mathrm{TP}$ signaling functions physiologically.

In addition to these actions in the immune system, $\mathrm{TxA}_{2}-\mathrm{TP}$ signaling exerts its action in a different system to combat against infection. Ashton et al. (2007) found that a parasite, T. cruzi, is itself capable of producing $\mathrm{TxA}_{2}$ and that $\mathrm{TP}(-/-)$ mice exhibited higher mortality and more severe cardiac pathology and parasitism than wild-type mice after infection. Bone marrow transfer experiments showed that TP receptor in somatic cells, and not bone marrowderived cells, is important for protection against parasites; how it functions remains to be determined.

4. Agonists and Antagonists. Thromboxane $\mathrm{A}_{2}$ and its precursor endoperoxide $\mathrm{PGH}_{2}$ both activate TP receptors. Both are very chemically unstable and therefore of limited practical use. For this reason, stable analogs were developed. The methanoepoxy analog 9-11-dideoxy-11 $\alpha, 9$ a-epoxymethano-prostaglandin $\mathrm{F}_{2 \mathrm{a}}$ (U-46619; Fig. 10) has been widely used. More potent compounds, such as I-BOP, are also available for study but may have an inconveniently slow onset and offset in isolated tissue preparations.

Both $\mathrm{PGH}_{2}$ and $\mathrm{TxA}_{2}$ activate TP receptors, the latter showing higher potency (Needleman et al., 1976; Svensson and Hamberg, 1976; Salzman et al., 1980; Armstrong et al., 1985; Vezza et al., 2002), although their chemical lability and the enzymatic conversion of $\mathrm{PGH}_{2}$ to $\mathrm{PGD}_{2}, \mathrm{PGE}_{2}, \mathrm{PGF}_{2 \alpha}$, and $\mathrm{PGI}_{2}$ complicate these measurements (Hornberger and Patscheke, 1989). Stabilization of the $\mathrm{TxA}_{2}$ ring structure by difluoro substitution at $\mathrm{C} 10$ yields a potent TP agonist (Morinelli et al., 1989). However, the majority of stable TP agonists derive from substitution of one or both ring oxygens in $\mathrm{PGH}_{2}$ or $\mathrm{TxA}_{2}$ with carbon (Fig. 10). U-46619 (the most commonly used standard agonist), 9,11-azo $\mathrm{PGH}_{2}$ and stable $\mathrm{TxA}_{2}$ analogs behave as full agonists on isolated vascular and respiratory smooth muscle and human platelet preparations (Tymkewycz et al., 1991), as does the cyclic carbonate AGN-191976 (Fig. 10) (Krauss et al., 1996). U-44069, 9,11etheno $\mathrm{PGH}_{2}$, pinane $\mathrm{TxA}_{2}$, and carbocyclic TxA 2 often show partial agonism on smooth muscle preparations; that is, they show a reduced maximum response and block the action of U-46619 without affecting the action of other contractile ago- 
nists (e.g., phenylephrine or histamine) (Jones et al., 1982; Krauss et al., 1996). They may also show partial agonism on human platelets, but results from this assay system must be interpreted cautiously. Thus, there are three distinct, concentration-dependent, functionally related components of "platelet activation," namely shape change, primary (reversible) aggregation, and secondary aggregation as a result of release of mediators, including $\mathrm{PGH}_{2} / \mathrm{TxA}_{2}$ from de novo synthesis. The generated $\mathrm{PGH}_{2}$ could spontaneously decay to $\mathrm{PGE}_{2}$ and/or $\mathrm{PGD}_{2}$, with corresponding activation of $\mathrm{EP}_{3}$ receptors enhancing platelet activation (Oelz et al., 1977; Matthews and Jones, 1993) and $\mathrm{DP}_{1}$ receptors inhibiting platelet activation (Keery and Lumley, 1988) through changes in cAMP levels (see Gresele et al., 1988). True TP partial agonists could elicit either submaximal shape change or complete shape change and reversible aggregation, as is the case for 9,11-etheno $\mathrm{PGH}_{2}$ (Jones and Wilson, 1980). However, functional antagonism may also modulate the response as in the case of carbocyclic $\mathrm{TxA}_{2}$, which weakly raises the cyclic AMP level (possibly through activation of sensitive $\mathrm{DP}_{1}$ and/or IP systems) sufficient to suppress the maximum aggregation response (Armstrong et al., 1985). Finally, COX/thromboxane synthase involved in the release reaction may be inhibited by $\mathrm{PGH} / \mathrm{TxA}$ analogs (Wlodawer et al., 1971). Taking these issues into account, Krauss et al. (1996) identified the C1-alcohol derivative AGN-192903 (Fig. 10) as an agent that behaved as a potent full agonist on rat aorta but only caused shape change in human platelets at high concentration. The authors discuss their findings in relation to the identification of two TP receptor subtypes (Takahara et al., 1990; Furci et al., 1991), one of which elicits smooth muscle contraction and platelet shape change, whereas the other elicits platelet aggregation (see also section on identification of $\operatorname{TP} \alpha$ and $\operatorname{TP} \beta$ subtypes).

Analogs with a 7-oxabicyclo[2.2.1]heptane ring system (Fig. 10, bottom right) were prepared as TP agonists on the basis of a favorably directed ring oxygen (Sprague et al., 1985). Of the eight possible $\alpha$-oxy isomers, the "natural" $8 \alpha, 12 \beta, 15 S$ analog (SQ-26655; Fig. 10) is a potent TP agonist. Introduction of a 16- $p$-halophenoxy moiety into the $\omega$ terminus, as in EP-171 (Fig. 10) (Wilson et al., 1988) and I-BOP (Sessa et al., 1990), markedly enhances TP agonism. Near-maximal shape change of the human platelet elicited by EP-171 at $0.1 \mathrm{nM}$ is only slowly reversed by a high concentration of TP antagonist, reflecting the slow dissociation of this agonist from the TP receptor (Jones et al., 1989). $\left.{ }^{125} \mathrm{I}\right] \mathrm{BOP}$ is a useful radioligand for the TP receptor (Morinelli et al., 1989). The $8 \alpha, 12 \alpha, 15 R$ analog retains TP agonism, whereas the $8 \alpha, 12 \alpha, 15 S$ analog shows a switch to TP antagonism (Sprague et al., 1985) and was the starting point for potent and selective TP antagonists such as SQ-29548 and BMS-180291 (ifetroban; Fig. 11). The $8 \beta, 12 \alpha, 15 S$ analog shows broad inhibitory activity on human platelets, which was subsequently attributed to $\mathrm{DP}_{1}$ agonism (see Fig. 11).

TP antagonist design has been ongoing for more than 2 decades. Many potent and selective TP antagonists of diverse structure have been synthesized (Jones et al., 2009). Partial agonism is prevalent among carba-ring analogs of $\mathrm{PGH}_{2}$ or $\mathrm{TxA}_{2}$ (see Wilson and Jones, 1985), and further modification of the $\omega$-chain often leads to pure antagonism. A prime example is I-SAP (Fig. 11), which contains a pinane ring akin to the dioxabicyclo [3.1.1] heptane ring of $\mathrm{TxA}_{2}$ and trans orientation of the side chains (Naka et al., 1992). In other prostanoid-like antagonists, a cis orientation of the side chains affords high affinity as in GR-32191 (Lumley et al., 1989) and BMS-180291(Ogletree et al., 1993) (Fig. 11). A benzenesulfonamido moiety present in I-SAP features in simpler TP antagonist molecules, such as BM-13505 (daltroban; Yanagisawa et al., 1987), Z-335 (Tanaka et al., 1998) and S-18886 (terutroban; Fig. 11) (Cimetière et al., 1998);

\section{TP antagonists}
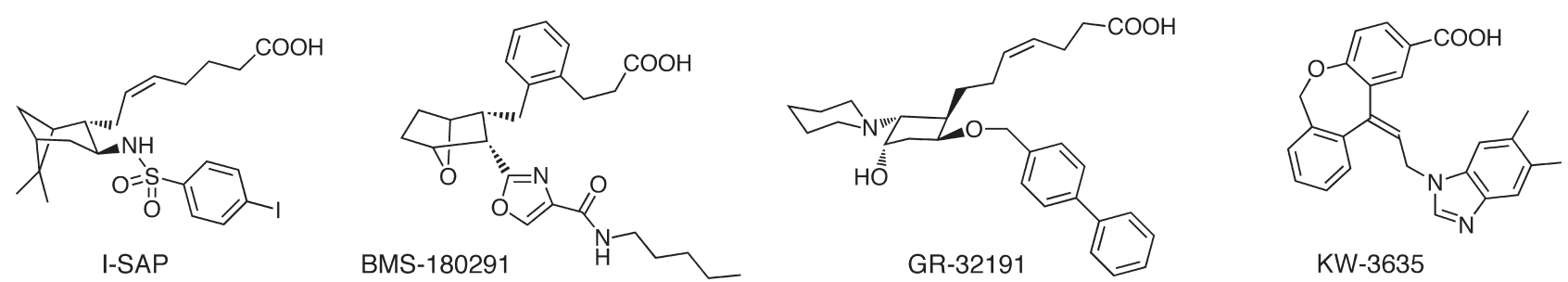

KW-3635
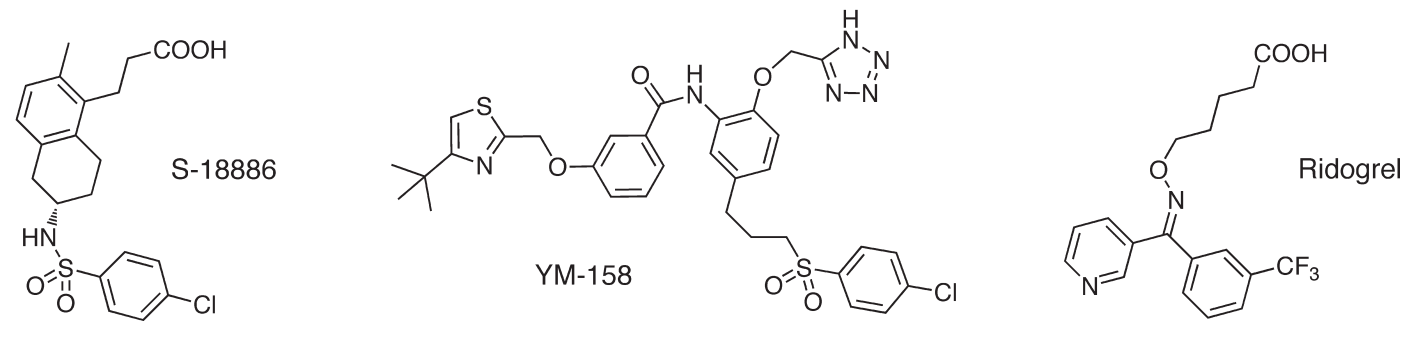

FIG. 11. Structures of representative antagonists for the prostanoid TP receptor. 
the distance from the C1-carboxylate group seems to be critical. 14,15-Epoxy-eicosatrienoic acid has been claimed to be an endogenous TP antagonist; it shows modest affinity for human $\mathrm{DP}_{1}, \mathrm{FP}$, and TP receptors $\left(K_{\mathrm{i}}=6.1,5.3\right.$, and $\left.3.2 \mu \mathrm{M}\right)$ with lower affinity for other prostanoid receptors ( $\geq 13 \mu \mathrm{M})$ (Behm et al., 2009).

For many of these compounds, surmountable antagonism on isolated smooth muscle preparations points toward reversible-competitive mechanism, and conventional Schild analysis confirms this classification. However, a slow approach to steady-state block by highaffinity antagonists can confound $\mathrm{p} A_{2}$ estimation. Under the Cheng-Prusoff inhibition-curve protocol, 6-(2-(2chlorophenyl-4-hydroxyphenyl)-1,3-dioxan-5-yl)hexenoic acid (ICI-192605) (Brewster et al., 1988) had not reached steady-state block on guinea pig aorta after 90-min incubation ( $\mathrm{p} A_{2}=10.25$ ) (Jones et al., 2008). With higher affinity TP antagonists in human and rat platelet assays, surmountability is seen for the shapechange response, whereas insurmountability occurs for aggregation. This profile is still compatible with a reversible-competitive mechanism, because slow dissociation of the high-affinity antagonist from the TP receptor retards agonist (U-46619) occupancy in the early stage of the aggregation response, thereby favoring the disaggregation process and insurmountability; in contrast, the nonfading shape-change response affords a truer measure of dose ratios at steady state (Armstrong et al., 1985; Jones et al., 1989; Lumley et al., 1989; Tymkewycz et al., 1991; Ogletree et al., 1993).

There has been much debate about the existence of different TP receptor subtypes in platelet and vascular smooth systems (Mais et al., 1985, 1988; Swayne et al., 1988; Morinelli et al., 1989; Masuda et al., 1991; Tymkewycz et al., 1991; Folger et al., 1992). It is certainly clear that species heterology exists; for example, higher affinities are often found for human and rat platelet TP receptors compared with rabbit platelet $\mathrm{TP}$ receptors (Tymkewycz et al., 1991). However, the situation may be more complex. There is evidence for two saturable binding sites for TP agonists on human platelets using several radioligands (Armstrong et al., 1983; Pollock et al., 1984; Ahn et al., 1988; Hedberg et al., 1988). The highaffinity site was associated with the platelet shape change (and increase in cytosolic $\mathrm{Ca}^{2+}$ ), whereas the lower-affinity site was associated with aggregation (and activation of PLC) (Dorn, 1989; Takahara et al., 1990). ${ }^{3}$ H-labeled GR-32191 (vapiprost) was bound reversibly to the "shape change site" and irreversibly to the "aggregation site" (Takahara et al., 1990). Given the structure of GR-32191 (Fig. 11), it is unlikely that covalent bonding is involved. Exposure of human platelets to GR32191 for $30 \mathrm{~min}$ resulted in approximately $50 \%$ loss of binding sites for either $\left[{ }^{3} \mathrm{H}\right] \mathrm{GR}-32191$ or $\left[{ }^{3} \mathrm{H}\right] \mathrm{SQ}-29548$, whereas neither SQ-29548 nor BM-13177 (sulotroban) affected $B_{\text {max }}$. It was speculated that GR-32191 binds to internalized TP receptors (Armstrong et al., 1983). 4[2- (4-azido-benzenesulfonylamino)-ethyl[phenoxyacetic acid), a light-activated, covalent-binding TP antagonist, also discriminated these platelet sites by blocking aggregation but not shape change induced by U-46619 (Zehender et al., 1988). Two TP receptor isoforms (TP $\alpha$ and $\mathrm{TP} \beta$ ) have been identified using a human umbilical vein endothelial cDNA library (Raychowdhury et al., 1994), and mRNAs for the $\alpha$ and $\beta$ isoforms have been detected in human platelets (Hirata et al., 1996). However, these isoforms, which arise by alternative gene splicing and differ only in their cytoplasmic tails, do not show the ligand discrimination typical of the high- and lowaffinity binding sites. Finally, only the TP $\alpha$ isoform was found in human platelets (Habib et al., 1999).

TP antagonism associated with IP agonism, TX synthase inhibition and LT receptor antagonism in the same molecule are all known. The bicyclo[2.2.2] octene $\mathrm{PGH}_{2}$ analog EP-157 (Figs. 8 and 11) activates IP receptors in both platelet and vascular systems (Armstrong et al., 1986, 1989; Jones et al., 1993). The presence of a diarylhetero(cyclic) moiety in the $\omega$-chain is crucial (Jones et al., 1993). Similar properties were found for octimibate, which lacks a prostanoid ring system (Merritt et al., 1991a,b) and is a member of a series of nonprostanoid prostacyclin mimetics (Meanwell et al., 1994; Seiler et al., 1997). Some of these agents also inhibit (nonprostanoid) Gq-PLC-driven responses (Chow et al., 2001).

Combined TP antagonism/thromboxane synthase inhibition usually requires the presence of a $(N)$-imidazole (dazoxiben; Randall et al., 1981) or a pyridin-3-yl group (isbogrel; Imura et al., 1990; ridogrel, Hoet et al., 1990; Fig. 11) to combine with the heme site of the synthase (see Hsu et al., 1999). Existing TP antagonists have also been modified to include similar reactive moieties: ZD1542 (Brownlie et al., 1993), a relative of ICI-192605, contains a pyridin-3-yl group and GR-83783 (Campbell et al., 1991a), a relative of GR-32191, has a 4-(pyridin3-yl)-phenyl moiety. In addition, sulotroban/daltroban moieties have been combined with ridogrel/isbogrel moieties (Campbell et al., 1991b; CGS-22652 (Bhagwat et al., 1993; Soyka et al., 1994) and the whole or part of the ICI-192605 nucleus has been tethered to either a dazoxiben or an isbogrel nucleus (Ackerley et al., 1995). More recently, compounds with dual antagonist properties have been designed. For example, (2-( $N$-(4-(4-chlorobenzenesulfonylamino)butyl)- $N$-(3-(4-isopropylthiazol-2yl)methoxy)benzyl)sulfamoyl)benzoic acid (KP-496) (Mizutani et al., 2008; Ishimura et al., 2009) and YM158 (Fig. 11) (Arakida et al., 1998) are dual TP/cysteinyl leukotriene antagonists.

5. Therapeutics. The original purpose for designing TP antagonists was as cardiovascular therapy but lowdose aspirin has proven a more economically viable proposition (Jones et al., 2009). Nevertheless, even recently the TP antagonist terutroban was shown to exhibit superior antithrombotic activity compared with as- 
pirin in humans (Bal Dit Sollier et al., 2009). Another early indication for TP antagonists was asthma, which met with some clinical success, albeit limited (Rolin et al., 2006; Jones et al., 2009). Other early indications do not seem to have met with any clinical success. These include cancer, glomerulonephritis, allergic rhinitis, inflammatory bowel disease, septic shock, and diabetes (Jones et al., 2009). The unique role of the $\mathrm{TP} \beta$ receptor isoform in bladder cancer and the delayed onset and prolonged survival afforded by TP antagonist treatment in mice transfected with bladder cancer cells (Moussa et al., 2008) holds promise for therapeutic utility in at least one form of cancer.

There are other potential uses for TP antagonists that have emerged more recently. The TP antagonist seratrodast seems to possess antitussive properties (Xiang et al., 2002; Ishiura et al., 2003). Ramatroban has been reported to attenuate cough in subjects with cough variant asthma (Kitamura et al., 2003a). The potential use of TP antagonists for treating preterm labor is not new but deserves new impetus based on recent findings on the human myometrium that $\mathrm{PGF}_{2 \alpha}$ responsiveness, but not $\mathrm{TxA}_{2}$ responsiveness, of the uterus is lost during labor (Fischer et al., 2008). The most recent potential uses for TP antagonists are listed in Table 10.

\section{F. $\mathrm{DP}_{2}$ Receptors (CRTR2)}

1. Second Messenger Signaling. Three independent but convergent research pathways led to the discovery of the $\mathrm{DP}_{2}$ (CRTH2) receptor subtype. A new surface marker on human Th2 cells in vivo (Nagata et al., 1999) was termed the "chemoattractant receptor-homologous molecule expressed on Th2 cells" (CRTH2). This proved to be identical to the orphan $\mathrm{G}$ protein-coupled receptor GPR44 (Marchese et al., 1999), which had sequence homology similar to that of typical chemoattractant receptors. Nevertheless, the naturally occurring ligand for the CRTH2 receptor was subsequently found to be $\mathrm{PGD}_{2}$ (Hirai et al., 2001; Monneret et al., 2001), and the term $\mathrm{DP}_{2}$ was introduced. There are now three descriptors: CRTH2, $\mathrm{DP}_{2}$, GPR44, with license for upper/lower case and subscripted/nonsubscripted variations. It has been suggested that the widespread distribution of this recep- tor, which extends far beyond the immune system, makes placement in the prostanoid receptor classification as $\mathrm{DP}_{2}$ more appropriate (Jones et al., 2009). The $\mathrm{DP}_{2}$ designation is used herein, given the topic of this review.

$\mathrm{DP}_{2}$ receptors are $\mathrm{G}_{\mathrm{i}}$-coupled, but signal transduction pathways have been subject to only limited investigation. $\mathrm{DP}_{2}$ receptor activation may result in pertussis toxin-sensitive decreases in cAMP levels (Sawyer et al., 2002; Gallant et al., 2007) and $\mathrm{Ca}^{2+}$ mobilization (Hirai et al., 2001; Sawyer et al., 2002). PI3K signaling has also been implicated in mediating $\mathrm{DP}_{2}$ effects (Hata et al., 2003; Xue et al., 2007). $\mathrm{DP}_{2}$ receptor trafficking has been studied and, interestingly, $\mathrm{PGD}_{2}$ induced $\mathrm{DP}_{2}$ but not $\mathrm{DP}_{1}$ receptor internalization (Gallant et al., 2007), which was decreased by inhibition of PKA and PKC. $\mathrm{DP}_{2}$ receptor internalization may be regulated by $\mathrm{PKC}$, GRK2, GRK3, GRK6 and arrestin-3 (Gallant et al., 2007) and the determinants located in the carboxyl terminus have been studied (Roy et al., 2010).

2. Distribution and Biological Functions. By combining functional data and transcription profiles together, it is clear that $\mathrm{DP}_{2}$ receptor expression is widespread. Northern blotting revealed high expression in the human stomach, small intestine, heart, and thymus; intermediate expression in the colon, spinal cord, and blood; and lower expression in the brain, skeletal muscle, and spleen (Sawyer et al., 2002). Functional studies demonstrate $\mathrm{DP}_{2}$ receptors present in smooth muscle, the cardiovascular system, the gastrointestinal tract, and the eye (Jones et al., 2009). The principal research focus during the past decade, after the cloning of $\mathrm{DP}_{2}$, however, has been inflammation.

$\mathrm{PGD}_{2}$ has long been known to cause eosinophil infiltration but with a pharmacological profile inconsistent with $\mathrm{DP}_{1}$ receptor mediation (Woodward et al., 1990, $1993 \mathrm{~b})$. The subsequent cloning of the receptor (Marchese et al., 1999; Nagata et al., 1999) and discovery of $\mathrm{DP}_{2}$ receptor as a Th2 cell chemoattractant (Hirai et al., 2001) provided important new dimensions. The role of $\mathrm{DP}_{1}$ and $\mathrm{DP}_{2}$ receptors in immunology and inflammation has been the subject of more than one review (Herlong and Scott, 2006; Kostenis and Ulven,

TABLE 10

Recently identified potential therapeutic application of TP antagonists

\begin{tabular}{|c|c|c|c|c|c|c|}
\hline Antagonist & Route & Dose & Species & Experimental Model & Indication & Reference \\
\hline GR-32191 (vapiprost) & $\begin{array}{l}\text { Oral } \\
\text { (drinking water) }\end{array}$ & $1 \mathrm{mg} \cdot \mathrm{kg}^{-1} \cdot \mathrm{day}^{-1}$ & Mouse & STZ diabetes & $\begin{array}{l}\text { Diabetic } \\
\text { retinopathy }\end{array}$ & Wright et al., 2009 \\
\hline KP-496 & Intratracheal & $\begin{array}{l}30 \text { and } 100 \mu \mathrm{m} / \\
\text { animal }\end{array}$ & Rat & $\begin{array}{l}\text { Sephadex induced } \\
\text { airway } \\
\text { inflammation }\end{array}$ & Asthma & Ishimura et al., 2009 \\
\hline KP-496 & Intranasal & $0.003-0.05 \%$ & Guinea pig & $\begin{array}{l}\text { Ovalbumin, Japanese } \\
\text { cedar pollen } \\
\text { allergy }\end{array}$ & Allergic rhinitis & Mizutani et al., 2008 \\
\hline ONO-3708 & Intradermal & 0.1 and $1 \mathrm{nmol} / \mathrm{site}$ & Mouse & $\begin{array}{l}\mathrm{TxA}_{2} \text {-induced } \\
\text { scratching }\end{array}$ & Pruritus & Andoh et al., 2007 \\
\hline S18886 (terutroban) & Oral & 10 and $30 \mathrm{mg} / \mathrm{kg}$ & Rat & $\begin{array}{l}\text { Uninephrectomized } \\
\text { obese Zucker rats }\end{array}$ & Type 2 diabetes & Sebeková et al., 2007 \\
\hline
\end{tabular}

STZ, streptozotocin; ONO-3708, (1S-(1 $\left.\left.\alpha, 2 \beta(Z), 3 \alpha\left(S^{*}\right), 5 \alpha\right)\right)$-7-(3-((cyclopentylhydroxyacetyl)amino)-6,6-dimethylbicyclo(3.1.1)hept-2-yl)-5-heptenoic acid. 
2006; Pettipher, 2008) and this topic is therefore discussed only briefly.

The discovery of $\mathrm{PGD}_{2}$ as an activator of Th2 lymphocytes via $\mathrm{DP}_{2}$ (CRTH2) receptors implicated them in immune regulation. The polarization of lymphocytes to the Th-2 phenotype is typically implicated in the development of allergic responses such as asthma and atopic dermatitis. $\mathrm{DP}_{2}$ receptor expression in $\mathrm{CD} 4^{+} \mathrm{T}$ cells is low in healthy humans but enhanced in atopic subjects and correlates with the severity of the disease (Kostenis and Ulven, 2006). In addition to recruitment of Th2 cells, $\mathrm{DP}_{2}$ receptor activation results in the production of cytokines such as IL-4, IL-5, IL-9, and IL-13 (Honda et al., 2003; Gallant et al., 2005; Xue et al., 2005; Kostenis and Ulven, 2006 Herlong and Scott, 2006). Recruitment and activation of eosinophils is a central feature of allergic responses, and they also express $\mathrm{DP}_{2}$ receptors. Activation of $\mathrm{DP}_{2}$ receptors leads to chemotaxis and degranulation of eosinophils (Gervais et al., 2001; Hirai et al., 2001; Monneret et al., 2001; Sugimoto et al., 2003; Böhm et al., 2004). $\mathrm{DP}_{2}$ receptor stimulation produces eosinophil infiltrates in living animals, and these are located at sites typically associated with allergy, such as the conjunctiva (Woodward et al., 1990, 1993b), cornea (Fujishima et al., 2005), lung (Almishri et al., 2005; Shiraishi et al., 2005; Spik et al., 2005), and skin (Spik et al., 2005). An important role for $\mathrm{DP}_{2}$ receptors in chronic cutaneous inflammation was revealed in $\mathrm{DP}_{2}$-deficient mice (Satoh et al., 2006). Prostaglandin $\mathrm{D}_{2}$ also activates basophils via $\mathrm{DP}_{2}$ receptors (Hirai et al., 2001; Cossette et al., 2007). A final comment on the roles of $\mathrm{DP}_{1}$ and $\mathrm{DP}_{2}$ in regulating allergy/inflammation (Kostenis and Ulven, 2006; Pettipher, 2008): the employment of a $\mathrm{DP}_{2}$ antagonist may switch $\mathrm{PGD}_{2}$ from a pro- to an antiinflammatory mediator in many instances.

Although widely distributed, notably in the gastrointestinal tract (Sawyer et al., 2002), the focus has been almost exclusively on leukocytes. Studies in the eye demonstrate that even within the context of allergic inflammation, $\mathrm{DP}_{2}$ receptor stimulation produces more than leukocyte activation. Thus, $\mathrm{DP}_{2}$ receptors are also associated with goblet cell depletion and increased microvascular permeability in the conjunctiva (Woodward et al., 1990, 1993b). In human retinal pigmented epithelial cells, $\mathrm{DP}_{2}$ receptors induce heme oxygenase- 1 expression (Satarug et al., 2008): these cells are essential for photoreceptor survival. $\mathrm{DP}_{2}$ has been suggested as a therapeutic target for delaying the onset of age-related macular degeneration and cerebral malaria (Satarug et al., 2008). Both DP receptor subtypes have been implicated in astrogliosis and demyelination and involved in the neuroinflammatory effects of $\mathrm{PGD}_{2}$ (Mohri et al., 2006).

3. Gene Deletion Studies. $\mathrm{DP}_{2}$ knockout mice were generated independently by two groups (Chevalier et al., 2005; Satoh et al., 2006), and apparently different phenotypes were reported for these two lines. Chevalier et al. (2005) subjected their $\mathrm{DP}_{2}$-deficient mice to the OVAinduced allergic asthma model and found markedly increased eosinophil recruitment into the bronchoalveolar lavage (BAL) fluid of $\mathrm{KO}$ mice compared with WT mice. This was opposite to the known function of $\mathrm{DP}_{2}$ (CRTH2), in that it mediates the chemotactic action of $\mathrm{PGD}_{2}$ on eosinophils. Indeed, $\mathrm{DP}_{2}$ receptor stimulation has been shown to increase the degree of inflammation in mice (Spik et al., 2005). To examine this discrepancy, they found that IL-5 production by activated T cells from $\mathrm{DP}_{2}$-deficient mice in vitro was increased compared with that observed with wild-type cells. They suggested that $\mathrm{DP}_{2}$ indeed functions to facilitate allergy in situ at the site of inflammation, but that this receptor also regulates IL-5 production in the early phase of allergy development. On the other hand, Satoh et al. (2006) injected anti-dinitrophenyl-specific IgE into the skin of the ear lobe of their $\mathrm{DP}_{2}$-deficient mice, challenged them with $\mathrm{DNFB}$, and found that this type of IgE-induced dermatitis was significantly suppressed in $\mathrm{DP}_{2}$-deficient mice. They further showed that acute as well as chronic contact hypersensitivity was partially suppressed in $\mathrm{DP}_{2}$ deficient mice. In the Discussion section of their article, Satoh et al. (2006) commented that their $\mathrm{DP}_{2}$-deficient mice did not exhibit enhanced eosinophilia when subjected to the asthma model and that splenocytes from their KO mice did not show exaggerated IL-5 production on activation. The $\mathrm{DP}_{2}$-deficient mice that Satoh et al. (2006) generated consistently showed less inflammatory response in allergic dermatitis induced by cutaneous application of Japanese cedar pollen (Oiwa et al., 2008) or allergic rhinitis induced by repeated intranasal sensitization with Cry j 1, Japanese cedar pollen antigen (Nomiya et al., 2008). In the latter study, suppression of the increase in serum Cry $\mathrm{j}$ 1-specific IgE, a significant reduction of IL-4, and a slight reduction of IL-5 produced by draining lymph node cells in $\mathrm{DP}_{2}$-deficient mice were reported. Furthermore, Shiraishi et al. (2008) examined the role of $\mathrm{DP}_{2}$ in the poly I:C-induced enhancement of allergic inflammation in the OVA asthma model and found that although $\mathrm{DP}_{2}$-deficient mice showed a comparable inflammatory eosinophil infiltration in BAL to WT mice after OVA challenge alone, they showed complete loss of the poly I:C-induced enhancement. No difference was found in the number of neutrophils and lymphocytes or the amounts of IL-5 and IL-13 in the BAL between WT and $\mathrm{DP}_{2}$-deficient mice. In addition to these studies focusing on the role of $\mathrm{DP}_{2}$ in eosinophil recruitment in allergic reactions, Tajima et al. (2008) found enhanced production of $\mathrm{PGE}_{2}$ and $\mathrm{PGD}_{2}$ during macrophage activation with LPS and examined the role of $\mathrm{DP}_{2}$ in LPS-induced migration of peritoneal macrophages. They reported that LPS-mediated migration was significantly suppressed in macrophages from $\mathrm{DP}_{2}$ deficient mice.

4. Agonists and Antagonists. Potent and selective agonists for the $\mathrm{DP}_{2}$ receptor have been discovered or de- 
vised. Although no therapeutic utility for $\mathrm{DP}_{2}$ agonists has been established, they have provided invaluable pharmacological tools. Indeed, 13,14-dihydro,15-keto $\mathrm{PGD}_{2}$ was the first $\mathrm{DP}_{2}$ agonist to be discovered (Jones, 1976a,b), and over the past 3 decades, numerous unique natural ligands have been discovered (Pettipher, 2008). Synthetic selective $\mathrm{DP}_{2}$ agonists include $15 R$-methyl $\mathrm{PGD}_{2}$ (Monneret et al., 2003) and (9-((4-chlorophenyl)thio)-6-fluoro-2,3-dihydro$1 H$-pyrrolo(1,2- $a$ )indol-1-yl)acetic acid (L-888,607) (Gervais et al., 2005). It is also interesting that indomethacin is a $\mathrm{DP}_{2}$ agonist (Hirai et al., 2002).

Attaining selective $\mathrm{DP}_{2}$ agonism has mainly involved alterations to the 15 -hydroxyl group. The $15 S$ stereochemistry is atypically not crucial to $\mathrm{DP}_{2}$ agonism, with $15 R$ - and $15 R$-15-methyl $\mathrm{PGD}_{2}$ showing high $\mathrm{DP}_{2} / \mathrm{DP}_{1}$ selectivity (Jones, 1976,a,b, 1978; Monneret et al., 2001, 2003; Kim et al., 2005; Cossette et al., 2007). Moreover, 15-oxo structures (usually considered to be bioinactive products of 15-hydroxy PG dehydrogenase) retain high $\mathrm{DP}_{2}$ agonism. The biologically active form of 15-oxo $\mathrm{PGD}_{2}$ is not clear, however, because of its ready interconversion between 11,5-dioxo-13E-ene (as drawn in Fig. 2) and 11,15-dioxo-12-ene tautomers, of which there are two $E$ and $Z$ geometric isomers (Jones and Wilson, 1978); the corresponding conjugated enols (e.g., 11-oxo12,14-diene-15-hydroxy) may even be active given the high potency of $\Delta^{12}-\mathrm{PGJ}_{2}$ (Monneret et al., 2002). Saturation of the 13,14-double bond obviates this chemical lability (Jones, 1976a,b, 1978; Rangachari et al., 1995) as does conversion of the 15-oxo group to an ethylene ketal (Jones, 1978). Loss of the 15-oxygen function also results in retention of $\mathrm{DP}_{2}$ agonism, as in 15-deoxy$\Delta^{12,14}-\mathrm{PGJ}_{2}$ (Monneret et al., 2002). The variance allowed at $\mathrm{C} 15$ is not unlimited, however; $15 \mathrm{~S}$-15-methyl $\mathrm{PGD}_{2}$ shows only weak $\mathrm{DP}_{2}$ agonism (Monneret et al., 2003). Finally, replacement of the 11-oxo group in $\mathrm{PGD}_{2}$ by methylene essentially abolishes $\mathrm{DP}_{1}$ agonism and results in weak $\mathrm{DP}_{2}$ antagonism (Cossette et al., 2007).

$\mathrm{DP}_{2}$ antagonists have been a major focus for drug discovery in recent years. This has been reviewed (Jones et al., 2009) and therefore is not discussed in depth herein. Several distinct structural classes have been used as design templates. Indomethacin being a $\mathrm{DP}_{2}$ agonist (Hirai et al., 2002; Stubbs et al., 2002), indole acetic acids have been designed as antagonists. Likewise, the nonsteroidal anti-inflammatory drug fenclofenac was also used as a starting point for $\mathrm{DP}_{2}$ antagonist design (Jones et al., 2009). Additional scaffolds include tetrahydroquinolines (Jones et al., 2009) and thiazoleacetic acids (Grimstrup et al., 2010). Ramatroban (Bay U 3405) has played a central role in $\mathrm{DP}_{2}$ antagonist design, and elements of this core structure are incorporated into many structures (Jones et al., 2009), including recently described indole-based $\mathrm{DP}_{2}$ antagonists (Stearns et al., 2009). Ramatroban is also a TP antagonist and is marketed for the treatment of allergic rhinitis (Sugimoto et al., 2003). Development of ramatroban was first directed toward TP antagonism (McKenniff et al., 1988). Later studies revealed low-affinity $\mathrm{DP}_{2}$ antagonism ( $\mathrm{p} \mathrm{A}_{2}, 7.44$ ) (Sugimoto et al., 2003; Mathiesen et al., 2006). Modification of ramatroban led to TM-30089 (CAY-10471; Fig. 3), which has much higher $\mathrm{DP}_{2} / \mathrm{TP}$ selectivity (Ulven and Kostenis, 2005). In assays of guanosine $5^{\prime}-O-\left(3-\left[{ }^{35} \mathrm{~S}\right]\right.$ thio $)$ triphosphate binding/ inositol phosphate accumulation and $\mathrm{PGD}_{2}$-induced eosinophil shape change, ramatroban and the related TM30642 were surmountable competitive antagonists, whereas TM-30643 and TM-30089 (Ulven and Kostenis, 2005) show insurmountability. Indomethacin has also provided a template for $\mathrm{DP}_{2}$ blockers, based to some extent on its weak agonism for the $\mathrm{DP}_{2}$ receptor (Hirai et al., 2002; Stubbs et al., 2002). Many of these analogs are designed around an inverted indole template (Birkinshaw et al., 2006). This latter profile may be due to slow dissociation from the $\mathrm{DP}_{2}$ receptor (Mathiesen et al., 2006). The embodiment of both $\mathrm{DP}_{1}$ and $\mathrm{DP}_{2}$ receptor antagonism in a single molecule seems to offer a potentially more effective therapeutic approach (Pettipher, 2008). This approach for antiallergic drug design is in progress, with at least one positive outcome in the form of AMG 009 (Liu et al., 2009).

5. Therapeutics. A variety of $\mathrm{DP}_{2}$ antagonists have been claimed active in models of allergic rhinitis, asthma, and atopic dermatitis (Table 11). Lung inflammation resulting from smoke inhalation has recently reported to be reduced by a $\mathrm{DP}_{2}$ antagonist (Stebbins et al., 2010).

\section{G. Receptor Heterodimerization}

It is now established that $\mathrm{G}$ protein-coupled receptors exist as dimers (Prinster et al., 2005; Lohse, 2010) that are precoupled to heterotrimeric proteins in living cell membranes (Nobles et al., 2005). IP receptor precoupling to $G_{\mathrm{s}}$ has been shown in the cell membrane (Nobles et al., 2005). Heterodimerization allows the receptor to extend its repertoire of $\mathrm{G}$ protein coupling and ligand binding. IP/TP heterodimerization provides an example of both phenomena. In terms of second-messenger signaling, $\mathrm{PGI}_{2}$-like characteristics are conferred on $\mathrm{TxA}_{2}$ mimetics, which occurs as a robust cAMP response (Wilson et al., 2004). IP/TP heterodimerization creates a new binding site, which recognizes isoprostane $\mathrm{E}_{2}$ (Wilson et al., 2004). Heterodimerization of a prostanoid receptor with a member outside of the prostanoid family has been observed in the form of $\mathrm{EP}_{1} / \beta_{2}$-adrenoceptor dimerization (McGraw et al., 2006).

Heterodimerization of alternative mRNA splicing variants may also occur. Isoprostane responses were enhanced when $\mathrm{TP} \alpha$ and $\mathrm{TP} \beta$ were coexpressed (Wilson et al., 2007). Coexpression of the wild-type FP receptor and an alternative mRNA splicing variant resulted in ligand recognition that was quite different from wildtype FP receptors (Liang et al., 2008). Unlike FP receptors, wild-type/alternative FP/FP heterodimers were 
TABLE 11

Therapeutic applications of $\mathrm{DP}_{2}$ antagonists

\begin{tabular}{|c|c|c|c|c|c|c|}
\hline $\mathrm{DP}_{2}$ antagonist & Route & Dose & Species & Experimental Model & Indication & Reference \\
\hline & & $m g / k g$ & & & & \\
\hline AM 156 & Oral & 10 & Mouse & Smoke exposure & COPD & Stebbins et al., 2010 \\
\hline Compound 23 & Oral & 10 & Mouse & Ovalbumin allergy & Allergic rhinitis & Stearns et al., 2009 \\
\hline Compound A & Oral & 10 & Mouse & FITC-cutaneous allergy & Atopic dermatitis & Boehme et al., 2009 \\
\hline Ramatroban & Oral & 5 & Mouse & $\begin{array}{l}\text { Allergic (ovalbumin) eosinophilic } \\
\text { airway inflammation }\end{array}$ & Asthma & Uller et al., 2007 \\
\hline Ramatroban & Oral & 30 & Mouse & Cry ${ }_{J} 1$-induced pollinosis & Allergic rhinitis & Nomiya et al., 2008 \\
\hline TM-30089 & Oral & 5 & Mouse & $\begin{array}{l}\text { Allergic (ovalbumin) eosinophilic } \\
\text { airway inflammation }\end{array}$ & Asthma & Uller et al., 2007 \\
\hline
\end{tabular}

AM 156, (2'-((cyclopropanecarbonylethylamino)methyl)-6-methoxy-4'-trifluoromethyl-biphenyl-3-yl)acetic acid; COPD, chronic obstructive pulmonary disease; FITC, fluorescein isothiocyanate.

able to respond to the prostamide $\mathrm{F}_{2 \alpha}$ mimetic bimatoprost, and the antagonist AGN 211335 (Liang et al., 2008) blocked responses to bimatoprost but not $\mathrm{PGF}_{2 \alpha}$. This pharmacology was entirely consistent with that observed for the prostamide receptor in ocular cells and isolated tissue preparations (Liang et al., 2003; Woodward et al., 2003, 2007; Matias et al., 2004; Spada et al., 2005; Wan et al., 2007; Stamer et al., 2010). Therapeutic uses for the prostamide $\mathrm{F}_{2 \alpha}$ mimetic bimatoprost have extended beyond glaucoma to include trichomegaly (Tauchi et al., 2010). No potential medical uses for prostamide antagonists (Woodward et al., 2009) have been reported.

\section{Acknowledgments}

We thank L. Rubin for considerable assistance in preparing and formatting the manuscript and J. W. Wang for compilation and formation of Fig. 1.

\section{Authorship Contributions}

Wrote or contributed to the writing of the manuscript: Woodward, Jones, and Narumiya.

\section{References}

Abe H, Takeshita T, Nagata K, Arita T, Endo Y, Fujita T, Takayama H, Kubo M, and Sugamura K (1999) Molecular cloning, chromosome mapping and characterization of the mouse CRTH2 gene, a putative member of the leukocyte chemoattractant receptor family. Gene 227:71-77.

Abramovitz M, Adam M, Boie Y, Carrière M, Denis D, Godbout C, Lamontagne S, Rochette C, Sawyer N, Tremblay NM, et al. (2000) The utilization of recombinant prostanoid receptors to determine the affinities and selectivities of prostaglandins and related analogs. Biochim Biophys Acta 1483:285-293.

Abramovitz M, Boie Y, Nguyen T, Rushmore TH, Bayne MA, Metters KM, Slipetz DM, and Grygorczyk R (1994) Cloning and expression of a cDNA for the human prostanoid FP receptor. J Biol Chem 269:2632-2636.

Accomazzo MR, Cattaneo S, Nicosia S, and Rovati GE (2002) Bell-shaped curves for prostaglandin-induced modulation of adenylate cyclase: two mutually opposing effects. Eur J Pharmacol 454:107-114.

Ackerley N, Brewster AG, Brown GR, Clarke DS, Foubister AJ, Griffin SJ, Hudson JA, Smithers MJ, and Whittamore PR (1995) A novel approach to dual-acting thromboxane receptor antagonist/synthase inhibitors based on the link of 1,3 dioxane-thromboxane receptor antagonists and -thromboxane synthase inhibitors. J Med Chem 38:1608-1628.

Adams JW, Migita DS, Yu MK, Young R, Hellickson MS, Castro-Vargas FE, Domingo JD, Lee PH, Bui JS, and Henderson SA (1996) Prostaglandin $\mathrm{F}_{2 \alpha}$ stimulates hypertrophic growth of cultured neonatal rat ventricular myocytes. J Biol Chem 271:1179-1186.

Aguirre SA, Huang W, Prasanna G, and Jessen B (2009) Corneal neovascularization and ocular irritancy responses in dogs following topical ocular administration of an $\mathrm{EP}_{4}$-prostaglandin $\mathrm{E}_{2}$ agonist. Toxicol Pathol 37:911-920.

Ahluwalia A and Perretti M (1994) Anti-inflammatory effect of prostanoid in mouse and rat skin: evidence for a role of $\mathrm{EP}_{3}$-receptors. J Pharmacol Exp Ther 268: 1526-1531.

Ahmad AS, Ahmad M, de Brum-Fernandes AJ, and Doré S (2005) Prostaglandin EP4 receptor agonist protects against acute neurotoxicity. Brain Res 1066:71-77.

Ahmad AS, Saleem S, Ahmad M, and Doré S (2006a) Prostaglandin $\mathrm{EP}_{1}$ receptor contributes to excitotoxicity and focal ischemic brain damage. Toxicol Sci 89:265270 .

Ahmad AS, Zhuang H, Echeverria V, and Doré S (2006b) Stimulation of prostaglan- din $\mathrm{EP}_{2}$ receptors prevents NMDA-induced excitotoxicity. $J$ Neurotrauma 23: 1895-1903

Ahmadi S, Lippross S, Neuhuber WL, and Zeilhofer HU (2002) PGE(2) selectively blocks inhibitory glycinergic neurotransmission onto rat superficial dorsal horn neurons. Nature Neurosci 5:34-40.

Ahn CH, Wallace LJ, Miller DD, and Feller DR (1988) Use of $\left[{ }^{3} \mathrm{H}\right]$ trimetoquinol as a radioligand in human platelets: interaction with putative endoperoxide/ thromboxane A, receptor sites. Thromb Res 50:387-399.

Akaike A, Kaneko S, Tamura Y, Nakata N, Shiomi H, Ushikubi F, and Narumiya S (1994) Prostaglandin $\mathrm{E}_{2}$ protects cultured cortical neurons against N-methyl-Daspartate receptor-mediated glutamate cytotoxicity. Brain Res 663:237-243.

Akhter MP, Cullen DM, Gong G, and Recker RR (2001) Bone biomechanical properties in prostaglandin $\mathrm{EP}_{1}$ and $\mathrm{EP}_{2}$ knockout mice. Bone 29:121-125.

Ali FY, Egan K, FitzGerald GA, Desvergne B, Wahli W, Bishop-Bailey D, Warner TD, and Mitchell JA (2006) Role of prostacyclin versus peroxisome proliferationactivated receptor beta receptors in prostacyclin sensing by lung fibroblasts. Am $J$ Respir Cell Mol Biol 34:242-246.

Allan CJ and Halushka PV (1994) Characterization of human peripheral blood monocyte thromboxane $\mathrm{A}_{2}$ receptors. J Pharmacol Exp Ther 270:446-452.

Almirza WH, Dernison MM, Peters PH, van Zoelen EJ, and Theuvenet AP (2008) Role of the prostanoid FP receptor in action potential generation and phenotypic transformation of NRK fibroblasts. Cell Signal 20:2022-2029.

Almishri W, Cossette C, Rokach J, Martin JG, Hamid Q, and Powell WS (2005) Effects of prostaglandin $\mathrm{D}_{2}, 15$-deoxy- $\Delta^{12,14}$-prostaglandin $\mathrm{J}_{2}$, and selective $\mathrm{DP}_{1}$ and $\mathrm{DP}_{2}$ receptor agonists on pulmonary infiltration of eosinophils in brown Norway rats. J Pharmacol Exp Ther 313:64-69.

Alvarez R, Eglen RM, Chang LF, Bruno JJ, Artis DR, Kluge AF, and Whiting RL (1991) Stimulation of prostaglandin $\mathrm{D}_{2}$ receptors on human platelets by analogs of prostacyclin. Prostaglandins 42:105-119.

Amano H, Hayashi I, Endo H, Kitasato H, Yamashina S, Maruyama T, Kobayashi M, Satoh K, Narita M, Sugimoto Y, et al. (2003) Host prostaglandin $\mathrm{E}_{2}-\mathrm{EP}_{3}$ signaling regulates tumor-associated angiogenesis and tumor growth. $J$ Exp Med 197:221232

Amano H, Ito Y, Suzuki T, Kato S, Matsui Y, Ogawa F, Murata T, Sugimoto Y, Senior $\mathrm{R}$, Kitasato $\mathrm{H}$, et al. (2009) Roles of a prostaglandin E-type receptor, $\mathrm{EP}_{3}$, in upregulation of matrix metalloproteinase- 9 and vascular endothelial growth factor during enhancement of tumor metastasis. Cancer Sci 100:2318-2324.

An S, Yang J, So SW, Zeng L, and Goetzl EJ (1994) Isoforms of the $\mathrm{EP}_{3}$ subtype of human prostaglandin $\mathrm{E}_{2}$ receptor transduce both intracellular calcium and cAMP signals. Biochemistry 33:14496-14502.

Andoh T, Nishikawa Y, Yamaguchi-Miyamoto T, Nojima H, Narumiya S, and Kuraishi Y (2007) Thromboxane $\mathrm{A}_{2}$ induces itch-associated responses through TP receptors in the skin of mice. J Invest Derm 127:2042-2047.

Andrade da Costa BL, Kang KD, Rittenhouse KD, and Osborne NN (2009) The localization of $\mathrm{PGE}_{2}$ receptor subtypes in rat retinal cultures and the neuroprotective effect of the $\mathrm{EP}_{2}$ agonist butaprost. Neurochem Int 55:199-207.

Andreasson K (2010) Emerging roles of $\mathrm{PGE}_{2}$ receptors in models of neurological disease. Prostaglandins Other Lipid Mediat 91:104-112.

Angeli V, Faveeuw C, Roye O, Fontaine J, Teissier E, Capron A, Wolowczuk I, Capron M, and Trottein F (2001) Role of the parasite-derived prostaglandin $\mathrm{D}_{2}$ in the inhibition of epidermal Langerhans cell migration during schistosomiasis infection. J Exp Med 193:1135-1147.

Angeli V, Staumont D, Charbonnier AS, Hammad H, Gosset P, Pichavant M, Lambrecht BN, Capron M, Dombrowicz D, and Trottein F (2004) Activation of the D prostanoid receptor 1 regulates immune and skin allergic responses. J Immunol 172:3822-3829.

Ankem MK, Jerde TJ, Wilkinson ER, and Nakada SY (2005) Third prize: prostaglandin $\mathrm{E}(2)-3$ receptor is involved in ureteral contractility in obstruction. $J$ Endourol 19:1088-1091.

Ansari HR, Davis AM, Kaddour-Djebbar I, and Abdel-Latif AA (2003) Effects of prostaglandin $\mathrm{F}_{2 \alpha}$ and latanoprost on phosphoinositide turnover, myosin light chain phosphorylation and contraction in cat iris sphincter. $J$ Ocul Pharmacol Ther 19:217-231.

Ansari KM, Sung YM, He G, and Fischer SM (2007) Prostaglandin receptor EP2 is responsible for cyclooxygenase-2 induction by prostaglandin E2 in mouse skin. Carcinogenesis 28:2063-2068.

Anthony TL, Lindsey JD, and Weinreb RN (2002) Latanoprost's effects on TIMP-1 and TIMP-2 expression in human ciliary muscle cells. Invest Ophthalmol Vis Sci 43:3705-3711.

Aoi M, Aihara E, Nakashima M, and Takeuchi K (2004) Participation of prostaglan- 
din $\mathrm{E}$ receptor EP4 subtype in duodenal bicarbonate secretion in rats. Am $J$ Physiol Gastrointest Liver Physiol 287:G96-G103.

Arai I, Takano N, Hashimoto Y, Futaki N, Sugimoto M, Takahashi N, Inoue T, and Nakaike S (2004) Prostanoid $\mathrm{DP}_{1}$ receptor agonist inhibits the pruritic activity in NC/Nga mice with atopic dermatitis. Eur J Pharmacol 505:229-235.

Arai I, Takaoka A, Hashimoto Y, Honma Y, Koizumi C, Futaki N, Sugimoto M, Takahashi N, Inoue T, Nakanishi Y, et al. (2007) Effects of TS-022 a newly development prostanoid $\mathrm{DP}_{1}$ receptor agonist, on experimental pruritus, cutaneous barrier disruption and atopic dermatitis in mice. Eur J Pharmacol 556:207214.

Arai K, Ohno T, Saeki T, Mizuguchi S, Kamata K, Hayashi I, Saigenji K, Murata T, Narumiya S, and Majima M (2003) Endogenous prostaglandin I2 regulates the neural emergency system through release of calcitonin gene related peptide. Gut 52:1242-1249.

Arakida Y, Suwa K, Ohga K, Yokota M, Miyata K, Yamada T, and Honda K (1998) In vitro pharmacologic profile of YM158, a new dual antagonist for $\mathrm{LTD}_{4}$ and $\mathrm{TXA}_{2}$ receptors. J Pharmacol Exp Ther 287:633-639.

Araujo M and Welch WJ (2009) Cyclooxygenase 2 inhibition suppresses tubuloglomerular feedback: roles of thromboxane receptors and nitric oxide. Am J Physiol Renal Physiol 296:F790-F794.

Arbab F, Goldsby J, Matijevic-Aleksic N, Huang G, Ruan KH, and Huang JC (2002) Prostacyclin is an autocrine regulator in the contraction of oviductal smooth muscle. Hum Reprod 17:3053-3059.

Arimura A, Yasui K, Kishino J, Asanuma F, Hasegawa H, Kakudo S, Ohtani M, and Arita H (2001) Prevention of allergic inflammation by a novel prostaglandin receptor antagonist, S-5751. J Pharmacol Exp Ther 298:411-419.

Ariumi H, Takano Y, Masumi A, Takahashi S, Hirabara Y, Honda K, Saito R, and Kamiya $\mathrm{HO}$ (2002) Roles of the central prostaglandin $\mathrm{EP}_{3}$ receptors in cardiovascular regulation in rats. Neurosci Lett 324:61-64.

Armstrong JM, Boura AL, Hamberg M, and Samuelsson B (1976) A comparison of the vasodepressor effects of the cyclic endoperoxides $\mathrm{PGG}$ and $\mathrm{PGH}_{2}$ with those of $\mathrm{PGD}_{2}$ and $\mathrm{PGE}_{2}$ in hypertensive and normal rats. Eur $J$ Pharmacol 39:251-258.

Armstrong RA (1996) Platelet prostanoid receptors. Pharmacol Ther 72:171-191.

Armstrong RA, Jones RL, MacDermot J, and Wilson NH (1986) Prostaglandin endoperoxide analogues which are both thromboxane receptor antagonists and prostacyclin mimetics. Br J Pharmacol 87:543-551.

Armstrong RA, Jones RL, Peesapati V, Will SG, and Wilson NH (1985) Competitive antagonism at thromboxane receptors in human platelets. $\mathrm{Br} J$ Pharmacol 84: 595-607.

Armstrong RA, Jones RL, and Wilson NH (1983) Ligand binding to thromboxane receptors on human platelets: correlation with biological activity. Br J Pharmacol 79:953-964.

Armstrong RA, Lawrence RA, Jones RL, Wilson NH, and Collier A (1989) Functional and ligand binding studies suggest heterogeneity of platelet prostacyclin receptors. Br J Pharmacol 97:657-668.

Aronoff DM, Lewis C, Serezani CH, Eaton KA, Goel D, Phipps JC, Peters-Golden M, and Mancuso P (2009) E-prostanoid 3 receptor deletion improves pulmonary host defense and protects mice from death in severe Streptococcus pneumoniae infection. J Immunol 183:2642-2649.

Asada M, Iwahashi M, Obitsu T, Kinoshita A, Nakai Y, Onoda T, Nagase T, Tanaka M, Yamaura Y, Takizawa H, et al. (2010) 3-(2-Aminocarbonylphenyl)propanoic acid analogs as potent and selective $\mathrm{EP}_{3}$ receptor antagonists. Part 2: optimization of the side chains to improve in vitro and in vivo potencies. Bioorg Med Chem 18:1641-1658.

Ashton AW, Mukherjee S, Nagajyothi FN, Huang H, Braunstein VL, Desruisseaux MS, Factor SM, Lopez L, Berman JW, Wittner M, et al. (2007) Thromboxane $\mathrm{A}_{2}$ is a key regulator of pathogenesis during Trypanosoma cruzi infection. J Exp Med 204:929-940.

Asselin E, Drolet P, and Fortier MA (1997) Cellular mechanisms involved during oxytocin-induced prostaglandin $\mathrm{F}_{2 \alpha}$ production in endometrial epithelial cells in vitro: role of cyclooxygenase-2. Endocrinology 138:4798-4805.

Astle S, Thornton S, and Slater DM (2005) Identification and localization of prostaglandin $\mathrm{E}_{2}$ receptors in upper and lower segment human myometrium during pregnancy. Mol Hum Reprod 11:279-287.

Audoly LP, Rocca B, Fabre JE, Koller BH, Thomas D, Loeb AL, Coffman TM, and FitzGerald GA (2000) Cardiovascular responses to the isoprostanes $\mathrm{iPF}_{2 \alpha} \mathrm{III}$ and $\mathrm{PE}_{2}$-III are mediated via the thromboxane $\mathrm{A}_{2}$ receptor in vivo. Circulation 101: 2833-2840.

Audoly LP, Ruan X, Wagner VA, Goulet JL, Tilley SL, Koller BH, Coffman TM, and Arendshorst WJ (2001) Role of $\mathrm{EP}(2)$ and $\mathrm{EP}(3)$ receptors in control of murine renal hemodynamics. Am J Physiol Heart Circ Physiol 280:H327-H333.

Audoly LP, Tilley SL, Goulet J, Key M, Nguyen M, Stock JL, McNeish JD, Koller BH, and Coffman TM (1999) Identification of specific EP receptors responsible for the hemodynamic effects of $\mathrm{PGE}_{2}$. Am J Physiol 277:H924-H930.

Axelsson H, Lönnroth C, Wang W, Svanberg E, and Lundholm K (2005) Cyclooxygenase inhibition in early onset of tumor growth and related angiogenesis evaluated in $\mathrm{EP}_{1}$ and $\mathrm{EP}_{3}$ knockout tumor-bearing mice. Angiogenesis 8:339-348.

Ayer LM, Wilson SM, Traves SL, Proud D, and Giembycz MA (2008) 4,5-Dihydro$1 \mathrm{H}$-imidazol-2-yl)-[4-(4-isopropoxy-benzyl)-phenyl]-amine (RO1138452) is a selective, pseudo-irreversible orthosteric antagonist at the prostacyclin (IP)-receptor expressed by human airway epithelial cells: IP-receptor-mediated inhibition of CXCL9 and CXCL10 release. J Pharmacol Exp Ther 324:815-826.

Babaev VR, Chew JD, Ding L, Davis S, Breyer MD, Breyer RM, Oates JA, Fazio S, and Linton MF (2008) Macrophage EP4 deficiency increases apoptosis and suppresses early atherosclerosis. Cell Metab 8:492-501.

Bachteeva V, Fock E, Lavrova E, Nikolaeva S, Gambaryan S, and Parnova R (2007) Prostaglandin $\mathrm{E}_{2}$ inhibits vasotocin-induced osmotic water permeability in the frog urinary bladder by $\mathrm{EP}_{1}$-receptor-mediated activation of NO/cGMP pathway. Am J Physiol Regul Integr Comp Physiol 293:R528-R537.

Bahler CK, Howell KG, Hann CR, Fautsch MP, and Johnson DH (2008) Prostaglan- dins increase trabecular meshwork outflow facility in cultured human anterior segments. Am J Ophthalmol 145:114-119.

Bal Dit Sollier C, Crassard I, Simoneau G, Bergmann JF, Bousser MG, and Drouet L (2009) ) Effect of the thromboxane prostaglandin receptor antagonist terutroban on arterial thrombogenesis after repeated administration in patients treated for the prevention of ischemic stroke. Cerebrovasc Dis 28:505-513.

Banerjee AK, Tuffin DP, and Walker JL (1985) Pharmacological effects of (+/-)-11deoxy,16-phenoxy-prostaglandin E1 derivatives in the cardiovascular system. $\mathrm{Br} J$ Pharmacol 84:71-80.

Bär KJ, Natura G, Telleria-Diaz A, Teschner P, Vogel R, Vasquez E, Schaible HG, and Ebersberger A (2004) Changes in effect of spinal prostaglandin $\mathrm{E}_{2}$ during inflammation: prostaglandin $\mathrm{E}\left(\mathrm{EP}_{1}-\mathrm{EP}_{4}\right)$ receptors in spinal nociceptive processing of input from the normal or inflamed knee joint. J Neurosci 24:642-651.

Barraclough P, Bolofo ML, Giles H, Gillam J, Harris CJ, Kelly MG, Leff P, McNeill A, Robertson AD, Stepney RJ, et al. (1996) Synthesis of hexahydrocyclopentimidazol-2-(1H)-one derivatives displaying selective DP-receptor agonist properties. Bioorg Med Chem 4:81-90.

Bastien L, Sawyer N, Grygorczyk R, Metters KM, and Adam M (1994) Cloning, functional expression, and characterization of the human prostaglandin $\mathrm{E}_{2}$ receptor $\mathrm{EP}_{2}$ subtype. J Biol Chem 269:11873-11877.

Battersby S, Critchley HO, de Brum-Fernandes AJ, and Jabbour HN (2004) Temporal expression and signalling of prostacyclin receptor in the human endometrium across the menstrual cycle. Reproduction 127:79-86.

Behm DJ, Ogbonna A, Wu C, Burns-Kurtis CL, and Douglas SA (2009) Epoxyeicosatrienoic acids function as selective, endogenous antagonists of native thromboxane receptors: identification of a novel mechanism of vasodilation. J Pharmacol Exp Ther 328:231-239.

Belley M, Gallant M, Roy B, Houde K, Lachance N, Labelle M, Trimble LA, Chauret N, Li C, Sawyer N, et al. (2005) Structure-activity relationship studies on orthosubstituted cinnamic acids, a new class of selective $\mathrm{EP}_{3}$ antagonists. Bioorg Med Chem Lett 15:527-530.

Bhagwat SS, Gude C, Cohen DS, Dotson R, Mathis J, Lee W, and Furness P (1993) Thromboxane receptor antagonism combined with thromboxane synthase inhibition. 5 . Synthesis and evaluation of enantiomers of 8-[[(4-chlorophenyl)sulfonyl] amino]-4(3-pyridinylalkyl)octanoic acid. J Med Chem 36:205-210.

Billot X, Chateauneuf A, Chauret N, Denis D, Greig G, Mathieu MC, Metters KM, Slipetz DM, and Young RN (2003) Discovery of a potent and selective agonist of the prostaglandin $\mathrm{EP}_{4}$ receptor. Bioorg Med Chem Lett 13:1129-1132.

Bilson HA, Mitchell DL, and Ashby B (2004) Human prostaglandin $\mathrm{EP}_{3}$ receptor isoforms show different agonist-induced internalization patterns. FEBS Lett 572: 271-275.

Birkinshaw TN, Teague SJ, Beech C, Bonnert RV, Hill S, Patel A, Reakes S, Sanganee H, Dougall IG, Phillips TT, et al. (2006) Discovery of potent CRTh2 $\left(\mathrm{DP}_{2}\right)$ receptor antagonists. Bioorg Med Chem Lett 16:4287-4290.

Bito LZ (2001) A new approach to the medical management of glaucoma, from the bench to the clinic, and beyond: the Proctor Lecture. Invest Ophthalmol Vis Sci 42:1126-1133.

Blackman SC, Dawson G, Antonakis K, and Le Breton GC (1998) The identification and characterization of oligodendrocyte thromboxane $\mathrm{A}_{2}$ receptors. J Biol Chem 273:475-483.

Bley KR, Bhattacharya A, Daniels DV, Gever J, Jahangir A, O'Yang C, Smith S, Srinivasan D, Ford AP, and Jett MF (2006) RO1138452 and RO03244794: characterization of structurally distinct, potent and selective IP (prostacyclin) receptor antagonists. $\mathrm{Br} J$ Pharmacol 147:335-345.

Bley KR, Hunter JC, Eglen RM, and Smith JA (1998) The role of IP prostanoid receptors in inflammatory pain. Trends Pharmacol Sci 19:141-147.

Blindt R, Bosserhoff AK, vom Dahl J, Hanrath P, Schrör K, Hohlfeld T, and MeyerKirchrath J (2002) Activation of IP and $\mathrm{EP}_{3}$ receptors alters cAMP-dependent cell migration. Eur J Pharmacol 444:31-37.

Blouin M, Han Y, Burch J, Farand J, Mellon C, Gaudreault M, Wrona M, Lévesque JF, Denis D, Mathieu MC, et al. (2010) The discovery of 4-\{1-[(\{2,5-dimethyl-4-[4(trifluoromethyl)benzyl]-3-thienyl\}carbonyl)amino]cyclopropyl\}benzoic acid (MK2894 ), a potent and selective prostaglandin $\mathrm{E}_{2}$ subtype 4 receptor antagonist. J Med Chem 53:2227-2238.

Boehme SA, Franz-Bacon K, Chen EP, Sásik R, Sprague LJ, Ly TW, Hardiman G, and Bacon KB (2009) A small molecule CRTH2 antagonist inhibits FITC-induced allergic cutaneous inflammation. Int Immunol 21:81-93.

Böhm E, Sturm GJ, Weiglhofer I, Sandig H, Shichijo M, McNamee A, Pease JE, Kollroser M, Peskar BA, and Heinemann A (2004) 11-Dehydro-thromboxane $\mathrm{B}_{2}$, a stable thromboxane metabolite, is a full agonist of chemoattractant receptorhomologous molecule expressed on TH2 cells (CRTH2) in human eosinophils and basophils. J Biol Chem 279:7663-7670.

Boie Y, Sawyer N, Slipetz DM, Metters KM, and Abramovitz M (1995) Molecular cloning and characterization of the human prostanoid DP receptor. $J$ Biol Chem 270:18910-18916

Boie Y, Stocco R, Sawyer N, Slipetz DM, Ungrin MD, Neuschäfer-Rube F, Püschel GP, Metters KM, and Abramovitz M (1997) Molecular cloning and characterization of the four rat prostaglandin $\mathrm{E}_{2}$ receptor subtypes. Eur J Pharmacol 340:227-241. Boku K, Ohno T, Saeki T, Hayashi H, Hayashi I, Katori M, Murata T, Narumiya S, Saigenji K, and Majima M (2001) Adaptive cytoprotection mediated by prostaglandin I(2) is attributable to sensitization of CRGP-containing sensory nerves. Gastroenterology 120:134-143.

Boniface K, Bak-Jensen KS, Li Y, Blumenschein WM, McGeachy MJ, McClanahan TK, McKenzie BS, Kastelein RA, Cua DJ, and de Waal Malefyt R (2009) Prostaglandin $\mathrm{E}_{2}$ regulates Th17 cell differentiation and function through cyclic AMP and $\mathrm{EP}_{2} / \mathrm{EP}_{4}$ receptor signaling. J Exp Med 206:535-548.

Bos CL, Richel DJ, Ritsema T, Peppelenbosch MP, and Versteeg HH (2004) Prostanoids and prostanoid receptors in signal transduction. Int J Biochem Cell Biol 36:1187-1205.

Bradbury DA, Newton R, Zhu YM, El-Haroun H, Corbett L, and Knox AJ (2003) Cyclooxygenase-2 induction by bradykinin in human pulmonary artery smooth 
muscle cells in mediated by the cyclic AMP response element through a novel autocrine loop involving endogenous prostaglandin E2, E-prostanoid 2 (EP2), and EP4 receptors. J Biol Chem 278:49954-49964.

Brescia MR, Rokosz LL, Cole AG, Stauffer TM, Lehrach JM, Auld DS, Henderson I, and Webb ML (2007) Discovery and preliminary evaluation of 5-(4-phenylbenzyl)oxazole4-carboxamides as prostacyclin receptor antagonists. Bioorg Med Chem Lett 17:12111215.

Brewster AG, Brown GR, Foubister AJ, Jessup R, and Smithers MJ (1988) The synthesis of a novel thromboxane receptor antagonist 4(Z)-6-(2-o-chlorophenyl-4o-hydroxyphenyl-1,3-dioxan-cis-5-yl) hexenoic acid ICI 192605. Prostaglandins 36: 173-178.

Breyer RM, Emeson RB, Tarng JL, Breyer MD, Davis LS, Abromson RM, and Ferrenbach SM (1994) Alternative splicing generates multiple isoforms of a rabbit prostaglandin $\mathrm{E}_{2}$ receptor. J Biol Chem 269:6163-6169.

Brochhausen C, Neuland P, Kirkpatrick CJ, Nüsing RM, and Klaus G (2006) Cyclooxygenases and prostaglandin $\mathrm{E}_{2}$ receptors in growth plate chondrocytes in vitro and in situ-prostaglandin $\mathrm{E}_{2}$ dependent proliferation of growth plate chondrocytes. Arthritis Res Ther 8:R78.

Brodt-Eppley J and Myatt L (1999) Prostaglandin receptors in lower segment myometrium during gestation and labor. Obstet Gynecol 93:89-93.

Brouxhon S, Konger RL, VanBuskirk J, Sheu TJ, Ryan J, Erdle B, Almudevar A, Breyer RM, Scott G, and Pentland AP (2007) Deletion of prostaglandin $\mathrm{E}_{2} \mathrm{EP}_{2}$ receptor protects against ultraviolet-induced carcinogenesis, but increases tumor aggressiveness. $J$ Invest Derm 127:439-446

Brownlie RP, Brownrigg NJ, Butcher HM, Garcia R, Jessup R, Lee VJ, Tunstall S, and Wayne MG (1993) ZD1542, a potent thromboxane $\mathrm{A}_{2}$ synthase inhibitor and receptor antagonist in vitro. $\mathrm{Br} J$ Pharmacol 110:1600-1606.

Brueggemeier RW, Richards JA, Joomprabutra S, Bhat AS, and Whetstone JL (2001) Molecular pharmacology of aromatase and its regulation by endogenous and exogenous agents. J Steroid Biochem Mol Biol 79:75-84.

Brugger N, Kim NN, Araldi GL, Traish AM, and Palmer SS (2008) Pharmacological and functional characterization of novel $\mathrm{EP}$ and $\mathrm{DP}$ receptor agonists: $\mathrm{DP}_{1}$ receptor mediates penile erection in multiple species. J Sex Med 5:344-356.

Bryniarski K, Biedron R, Jakubowski A, Chlopicki S, and Marcinkiewicz J (2008) Anti-inflammatory effect of 1-methylnicotinamide in contact hypersensitivity to oxazolone in mice; involvement of prostacyclin. Eur $J$ Pharmacol 578:332-338.

Bunce KT, Clayton NM, Coleman RA, Collington EW, Finch H, Humphray JM Humphrey PP, Reeves JJ, Sheldrick RL, and Stables R (1991) GR63799X-a novel prostanoid with selectivity for $\mathrm{EP}_{3}$ receptors. Adv Prostaglandin Thromboxane Leukot Res 21A:379-382.

Burelout C, Thibault N, Levasseur S, Simard S, Naccache PH, and Bourgoin SG (2004) Prostaglandin $\mathrm{E}_{2}$ inhibits the phospholipase D pathway stimulated by formyl-methionyl-leucyl-phenylalanine in human neutrophils. Involvement of $\mathrm{EP}_{2}$ receptors and phosphatidylinositol 3-kinase $\gamma$. Mol Pharmacol 66:293-301.

Cameron KO, Lefker BA, Ke HZ, Li M, Zawistoski MP, Tjoa CM, Wright AS, DeNinno SL, Paralkar VM, Owen TA, et al. (2009) Discovery of CP-533536: an $\mathrm{EP}_{2}$ receptor selective prostaglandin $\mathrm{E}_{2}\left(\mathrm{PGE}_{2}\right)$ agonist that induces local bone formation. Bioorg Med Chem Lett 19:2075-2078.

Campbell IB, Collington EW, Finch H, Hayes R, Lumley P, Mills K, Robertson GM, Wharton K, and Watts IS (1991a) Synthesis and pharmacological evaluation of combined thromboxane receptor antagonist/synthase inhibitors: pyridinecontaining amino-prostanoids. Bioorg Med Chem Lett 1:695-698.

Campbell IB, Collington EW, Finch H, Hayes R, Lumley P, Mills NB, Robertson GM, and Watts IS (1991b) Synthesis and pharmacological evaluation of combined thromboxane receptor antagonist/synthase inhibitors: pyridine-containing sulfonamido acids. Bioorg Med Chem Lett 1:699-704.

Cao J, Shayibuzhati M, Tajima T, Kitazawa T, and Taneike T (2002) In vitro pharmacological characterization of the prostanoid receptor population in the non-pregnant porcine myometrium. Eur J Pharmacol 442:115-123.

Carrasco MP, Phaneuf S, Asbóth G, and López Bernal A (1996) Fluprostenol activates phospholipase $\mathrm{C}$ and $\mathrm{Ca}^{2+}$ mobilization in human myometrial cells. J Clin Endocrinol Metab 81:2104-2110.

Caspi RR (2006) Animal models of autoimmune and immune-mediated uveitis. Drug Discov Today Dis Models 3:3-9.

Castellone MD, Teramoto H, Williams BO, Druey KM, and Gutkind JS (2005) Prostaglandin E2 promotes colon cancer cell growth through a $\mathrm{G}_{\mathrm{s}}$-axin- $\beta$-catenin signaling axis. Science 310:1504-1510.

Cefalu JS, Zhu QM, Eggers AC, Kaan TK, Ho MJ, Jett MF, Cockayne DA, Ford AP, and Nunn PA (2007) Effects of the selective prostacyclin receptor antagonist RO3244019 on the micturition reflex in rats. J Urol 178:2683-2688.

Chang SH, Ai Y, Breyer RM, Lane TF, and Hla T (2005a) The prostaglandin $\mathrm{E}_{2}$ receptor $\mathrm{EP}_{2}$ is required for cyclooxygenase 2-mediated mammary hyperplasia. Cancer Res 65:4496-4499.

Chang SH, Liu CH, Wu MT, and Hla T (2005b) Regulation of vascular endothelial cell growth factor expression in mouse mammary tumor cells by the $\mathrm{EP}_{2}$ subtype of the prostaglandin $\mathrm{E}_{2}$ receptor. Prostaglandins Other Lipid Mediat 76:48-58.

Chell SD, Witherden IR, Dobson RR, Moorghen M, Herman AA, Qualtrough D, Williams AC, and Paraskeva C (2006) Increased EP4 receptor expression in colorectal cancer progression promotes cell growth and anchorage independence. Cancer Res 66:3106-3113.

Chemtob S and Peri KG (2006), inventors; Hospital Sainte-Justine, assignee. Peptide antagonists of prostaglandin $\mathrm{F}_{2 \alpha}$ receptor. U.S. patent 6,984,719. 2006 Jan 10

Chen BC, Liao CC, Hsu MJ, Liao YT, Lin CC, Sheu JR, and Lin CH (2006) Peptidoglycan-induced IL-6 production in RAW 264.7 macrophages is mediated by cyclooxygenase- $2, \mathrm{PGE}_{2} / \mathrm{PGE}_{4}$ receptors, protein kinase $\mathrm{A}, \mathrm{I} \kappa \mathrm{B}$ kinase, and NF- $\kappa \mathrm{B}$ Immunol 177:681-693.

Chen DB, Westfall SD, Fong HW, Roberson MS, and Davis JS (1998) Prostaglandin F2alpha stimulates the Raf/MEK1/mitogen-activated protein kinase signaling cascade in bovine luteal cells. Endocrinology 139:3876-3885.

Chen J, Champa-Rodriguez ML, and Woodward DF (1995) Identification of a pro- stanoid FP receptor population producing endothelium-dependent vasorelaxation in the rabbit jugular vein. Br J Pharmacol 116:3035-3041.

Chen J, Zhao M, He W, Milne GL, Howard JR, Morrow J, Hébert RL, Breyer RM, Chen J, and Hao CM (2008) Increased dietary $\mathrm{NaCl}$ induces renal medullary PGE production and natriuresis via the $\mathrm{EP}_{2}$ receptor. Am J Physiol Renal Physio 295:F818-F825.

Chen Q, Muramoto K, Masaaki N, Ding Y, Yang H, Mackey M, Li W, Inoue Y, Ackermann K, Shirota H, et al. (2010) A novel antagonist of the prostaglandin $\mathrm{E}_{2}$ $\mathrm{EP}_{4}$ receptor inhibits Th1 differentiation and Th17 expansion and is orally active in arthritis models. $B r J$ Pharmacol 160:292-310.

Cheng K, Wu TJ, Wu KK, Sturino C, Metters K, Gottesdiener K, Wright SD, Wan Z, O'Neill G, Lai E, et al. (2006) Antagonism of the prostaglandin $\mathrm{D}_{2}$ receptor suppresses nicotinic acid-induced vasodilation in mice and humans. Proc Nat Acad Sci USA 103:6682-6687.

Cheng Y, Austin SC, Rocca B, Koller BH, Coffman TM, Grosser T, Lawson JA, and FitzGerald GA (2002) Role of prostacyclin in the cardiovascular response to thromboxane $\mathrm{A}_{2}$. Science 296:539-541.

Cheon H, Rho YH, Choi SJ, Lee YH, Song GG, Sohn J, Won NH, and Ji JD (2006 Prostaglandin $\mathrm{E}_{2}$ augments IL-10 signaling and function. J Immunol 177:10921100.

Cherukuri DP, Chen XB, Goulet AC, Young RN, Han Y, Heimark RL, Regan JW, Meuillet E, and Nelson MA (2007) The $\mathrm{EP}_{4}$ receptor antagonist, L-161,982, block prostaglandin $\mathrm{E}_{2}$-induced signal transduction and cell proliferation in $\mathrm{HCA}^{-7}$ colon cancer cells. Exp Cell Res 313:2969-2979.

Cheung JO, Grant ME, Jones CJ, Hoyland JA, Freemont AJ, and Hillarby MC (2003) Apoptosis of terminal hypertrophic chondrocytes in an in vitro model of endochondral ossification. J Pathol 201:496-503.

Chevalier E, Stock J, Fisher T, Dupont M, Fric M, Fargeau H, Leport M, Soler S, Fabien S, Pruniaux MP et al. (2005) Cutting edge: chemoattractant receptorhomologous molecule expressed on Th2 cells plays a restricting role on IL-5 production and eosinophil recruitment. J Immunol 175:2056-2060.

Chiang $\mathrm{N}$ and Tai HH (1998) The role of $\mathrm{N}$-glycosylation of human thromboxane A receptor in ligand binding. Arch Biochem Biophys 352:207-213.

Chien EK and Macgregor C (2003) Expression and regulation of the rat prostaglandin $\mathrm{E}_{2}$ receptor type $4\left(\mathrm{EP}_{4}\right)$ in pregnant cervical tissue. Am J Obstet Gyneco 189:1501-1510.

Chollet A, Tos EG, and Cirillo R (2007) Tocolytic effect of a selective FP receptor antagonist in rodent models reveals an innovative approach to the treatment of preterm labor. BMC Pregnancy Childbirth 7 (Suppl 1):S16.

Chow KB, Jones RL, and Wise H (2003) Protein kinase A-dependent coupling of mouse prostacyclin receptors to $\mathrm{G}_{\mathrm{i}}$ is cell-type dependent. Eur $J$ Pharmacol 474 $7-13$.

Chow KB, Wong YH, and Wise H (2001) Prostacyclin receptor-independent inhibition of phospholipase $\mathrm{C}$ activity by non-prostanoid prostacyclin mimetics. $\mathrm{Br} J$ Pharmacol 134:1375-1384.

Chun KS, Lao HC, Trempus CS, Okada M, and Langenbach R (2009) The prostaglandin receptor $\mathrm{EP}_{2}$ activates multiple signaling pathways and beta-arrestin complex formation during mouse skin papilloma development. Carcinogenesis 30:1620-1627.

Cimetière B, Dubuffet T, Muller O, Descombes JJ, Simonet S, Laubie M, Verbeuren TJ, and Lavielle G (1998) Synthesis and biological evaluation of new tetrahydronaphthalene derivatives as thromboxane receptor antagonists. Bioorg Med Chem Lett 8:1375-1380.

Cipollone F, Fazia ML, Iezzi A, Cuccurullo C, De Cesare D, Ucchino S, Spigonardo F Marchetti A, Buttitta F, Paloscia L, et al. (2005) Association between prostaglandin E receptor subtype EP4 overexpression and unstable phenotype in atherosclerotic plaques in human. Arterioscler Thromb Vasc Biol 25:1925-1931.

Cirillo R, Tos EG, Page P, Missotten M, Quattropani A, Scheer A, Schwarz MK, and Chollet A (2007) Arrest of preterm labor in rat and mouse by an oral and selective nonprostanoid antagonist of the prostaglandin $\mathrm{F}_{2 \alpha}$ receptor (FP). Am J Obstet Gynecol 197:54.e1-54.e9.

Clark CA, Schwarz EM, Zhang X, Ziran NM, Drissi H, O'Keefe RJ, and Zuscik MJ (2005) Differential regulation of EP receptors isoforms during chondrogenesis and chondrocyte maturation. Biochem Biophys Res Commun 328:764-776.

Clark P, Rowland SE, Denis D, Mathieu MC, Stocco R, Poirier H, Burch J, Han Y, Audoly L, Therien AG, et al. (2008) MF498 [N-\{[4-(5,9-Diethoxy-6-oxo-6,8-dihydro7H-pyrrolo[3,4-g] quinolin-7-yl)-3-methylbenzyl] sulfonyl\}-2-(2-methoxyphenyl)acetamide], a selective $\mathrm{E}$ prostanoid receptor 4 antagonist, relieves joint inflammation and pain in rodent models of rheumatoid and osteoarthritis. J Pharmacol Exp Ther 325:425-434.

Clarke DL, Belvisi MG, Catley MC, Yacoub MH, Newton R, and Giembycz MA (2004) Identification in human airways smooth muscle cells of the prostanoid receptor and signalling pathway through which $\mathrm{PGE}_{2}$ inhibits the release of GM-CSF. $\mathrm{Br} J$ Pharmacol 141:1141-1150.

Claudino RF, Kassuya CA, Ferreira J, and Calixto JB (2006) Pharmacological and molecular characterization of the mechanisms involved in prostaglandin $\mathrm{E}_{2}$ induced mouse paw edema. J Pharmacol Exp Ther 318:611-618.

Clevers H (2006) Colon cancer-understanding how NSAIDs work. $N$ Engl J Med 354:761-763.

Coleman R and Middlemiss D (2009), inventors; Asterand UK Ltd, Coleman R, and Middlemiss D, assignees. Difluorobiphenylamide derivatives for the treatment of ocular hypertension. World patent WO/2009/098458.

Coleman RA, Grix SP, Head SA, Louttit JB, Mallett A, and Sheldrick RL (1994a) A novel inhibitory prostanoid receptor in piglet saphenous vein. Prostaglandins 47:151-168.

Coleman RA, Humphrey PPA, Kennedy I, and Lumley P (1984) Prostanoid receptors-the development of a working classification. Trends Pharmacol Sci 5:303306

Coleman RA, Kennedy I, and Sheldrick RL (1987) New evidence with selective agonists and antagonists for the subclassification of $\mathrm{PGE}_{2}$-senstive (EP) receptors. Adv Prostaglandin Thromboxane Leukot Res 17A:467-470. 
Coleman RA and Sheldrick RL (1989) Prostanoid-induced contractions of human bronchial smooth muscle is mediated by TP receptors. $\mathrm{Br} J$ Pharmacol 96:688692.

Coleman RA, Smith WL, and Narumiya S (1994b) International Union of Pharmacology classification of prostanoid receptors: properties, distribution, and structure of the receptors and their subtypes. Pharmacol Rev 46:205-229.

Collins PW, Pappo R, and Dajani EZ (1985) Chemistry and synthetic development of misoprostol. Dig Dis Sci 30 (11 Suppl):114S-117S.

Colombe L, Michelet JF, and Bernard BA (2008) Prostanoid receptors in anagen human hair follicles. Exp Derm 17:63-72.

Cossette C, Walsh SE, Kim S, Lee GJ, Lawson JA, Bellone S, Rokach J, and Powell WS (2007) Agonist and antagonist effects of 15R-prostaglandin (PG) $\mathrm{D}_{2}$ and 11methylene-PGD $\mathrm{D}_{2}$ on human eosinophils and basophils. J Pharmacol Exp Ther 320:173-179

Côté SC, Pasvanis S, Bounou S, and Dumais N (2009) CCR7-specific migration to CCL19 and CCL21 is induced by $\mathrm{PGE}_{2}$ stimulation in human monocytes: involvement of $\mathrm{EP}_{2} / \mathrm{EP}_{4}$ receptors activation. Mol Immunol 46:2682-2693.

Crawford KS, Kaufman PL, Hubbard WC, and Woodward DF (1992) The DPreceptor agonist SQ 27986 raises but does not lower intraocular pressure in ocular normotensive monkeys. J Glaucoma 1:94-99.

Crider JY, Griffin BW, and Sharif NA (1999) Prostaglandin DP receptors positively coupled to adenylyl cyclase in embryonic bovine tracheal (EBTr) cells: pharmacological characterization using agonists and antagonists. Br J Pharmacol 127:204210 .

Crowston JG, Lindsey JD, Aihara M, and Weinreb RN (2004) Effect of latanoprost on intraocular pressure in mice lacking the prostaglandin FP receptor. Invest Ophthalmol Vis Sci 45:3555-3559.

Crowston JG, Lindsey JD, Morris CA, Wheeler L, Medeiros FA, and Weinreb RN (2005) Effect of bimatoprost on intraocular pressure in prostaglandin FP receptor knockout mice. Invest Ophthalmol Vis Sci 46:4571-4577.

Crowston JG, Morris CA, Lindsey JD, and Weinreb RN (2007) Prostaglandin FP receptors do not contribute to 24-hour intraocular pressure variation in mice. Invest Ophthalmol Vis Sci 48:2095-2098.

Darius H, Michael-Hepp J, Thierauch KH, and Fisch A (1994) Inhibition of human platelets and polymorphonuclear neutrophils by the potent and metabolically stable prostaglandin $\mathrm{D}_{2}$ analog ZK 118.182. Eur J Pharmacol 258:207-213.

Davis RJ, Murdoch CE, Ali M, Purbrick S, Ravid R, Baxter GS, Tilford N, Sheldrick $\mathrm{RL}$, Clark KL, and Coleman $\mathrm{RA}(2004) \mathrm{EP}_{4}$ prostanoid receptor-mediated vasodilatation of middle cerebral arteries. Br J Pharmacol 141:580-585.

De Asua LJ, Clingan D, and Rudland PS (1975) Initiation of cell proliferation in cultured mouse fibroblasts by prostaglandin $\mathrm{F}_{2 \alpha}$. Proc Natl Acad Sci USA 72: $2724-2728$.

Denniss SG and Rush JW (2009) Impaired hemodynamics and endothelial vasomotor function via endoperoxide-mediated vasoconstriction in the carotid artery of spontaneously hypertensive rats. Am J Physiol Heart Circ Physiol 296:H1038-H1047.

Dey I, Lejeune M, and Chadee K (2006) Prostaglandin $\mathrm{E}_{2}$ receptor distribution and function in the gastrointestinal tract. Br J Pharmacol 149:611-623.

Di Cesare A, Del Piccolo P, Zacchetti D, and Grohovaz F (2006) $\mathrm{EP}_{2}$ receptor stimulation promotes calcium responses in astrocytes via activation of the adenylyl cyclase pathway. Cell Mol Life Sci 63:2546-2553.

Di Francesco L, Totani L, Dovizio M, Piccoli A, Di Francesco A, Salvatore T, Pandolfi A, Evangelista V, Dercho RA, Seta F, et al. (2009) Induction of prostacyclin by steady laminar shear stress suppresses tumor necrosis factor- $\alpha$ biosynthesis via heme oxygenase-1 in human endothelial cells. Circ Res 104:506-513.

Dogné JM, Hanson J, and Pratico D (2005) Thromboxane, prostacyclin and isoprostanes: therapeutic targets in atherogenesis. Trends Pharmacol Sci 26:639-644.

Doheny HC, O'Reilly MJ, Sexton DJ, and Morrison JJ (2007) THG113.31, a specific $\mathrm{PGF} 2 a l p h a$ receptor antagonist, induces human myometrial relaxation and $\mathrm{BKCa}$ channel activation. Reprod Biol Endocrinol 5:10.

Doherty GA, Byrne SM, Molloy ES, Malhotra V, Austin SC, Kay EW, Murray FE, and Fitzgerald DJ (2009) Proneoplastic effects of PGE2 mediated by $\mathrm{EP}_{4}$ receptor in colorectal cancer. BMC Cancer 9:207.

Doi Y, Minami T, Nishizawa M, Mabuchi T, Mori H, and Ito S (2002) Central nociceptive role of prostacyclin (IP) receptor induced by peripheral inflammation. Neuroreport 13:93-96.

Dong YJ and Jones RL (1982) Effects of prostaglandins and thromboxane analogues on bullock and dog iris sphincter preparations. Br J Pharmacol 76:149-155.

Dong YJ, Jones RL, and Wilson NH (1986) Prostaglandin E receptor subtypes in smooth muscle: agonist activities of stable prostacyclin analogues. $\mathrm{Br}$ J Pharmacol 87:97-107.

Donnini S, Finetti F, Solito R, Terzuoli E, Sacchetti A, Morbidelli L, Patrignani P, and Ziche $\mathrm{M}$ (2007) $\mathrm{EP}_{2}$ prostanoid receptor promotes squamous cell carcinoma growth through epidermal growth factor receptor transactivation and iNOS and ERK 1/2 pathways. FASEB J 21:2418-2430.

Dorn GW 2nd (1989) Distinct platelet thromboxane $\mathrm{A}_{2}$ /prostaglandin $\mathrm{H}_{2}$ receptor subtypes. A radioligand binding study of human platelets. J Clin Invest 84:18831891.

Dorn GW 2nd and Becker MW (1993) Thromboxane $\mathrm{A}_{2}$ stimulated signal transduction in vascular smooth muscle. J Pharmacol Exp Ther 265:447-456.

Ducharme Y, Blouin M, Carrière MC, Chateauneuf A, Côté B, Denis D, Frenette R, Greig G, Kargman S, Lamontagne S, et al. (2005) 2,3-Diarylthiophenes as selective $\mathrm{EP}_{1}$-receptor antagonists. Bioorg Med Chem Lett 15:1155-1160.

Duckworth N, Marshall K, and Clayton JK (2002) An investigation of the effect of the prostaglandin $\mathrm{EP}_{2}$ receptor agonist, butaprost, on the human isolated myometrium from pregnant and non-pregnant women. $J$ Endocrinol 172:263-269.

Dukes M, Russell W, and Walpole AL (1974) Potent luteolytic agents related to prostaglandin $\mathrm{F}_{2 \alpha}$. Nature 250:330-331.

Dumais N, Barbeau B, Olivier M, and Tremblay MJ (1998) Prostaglandin $E_{2}$ upregulates HIV-1 long terminal repeat-driven gene activity in T cells via NF- $\kappa \mathrm{B}$ dependent and -independent signaling pathways. J Biol Chem 273:27306-27314.
Duncan AM, Anderson LL, Funk CD, Abramovitz M, and Adam M (1995) Chromosomal localization of the prostanoids receptor gene family. Genomics 25:740-742

Durocher Y, Perret S, Thibaudeau E, Gaumond MH, Kamen A, Stocco R, and Abramovitz M (2000) A reporter gene assay for high-throughput screening of G-protein-coupled receptors stably or transiently expressed in HEK293 EBNA cells grown in suspension culture. Anal Biochem 284:316-326.

Echeverria V, Clerman A, and Doré S (2005) Stimulation of PGE receptors $\mathrm{EP}_{2}$ and $\mathrm{EP}_{4}$ protects cultured neurons against oxidative stress and cell death following beta-amyloid exposure. Eur J Neurosci 22:2199-2206.

Eglen RM and Whiting RL (1988) The action of prostanoid receptor agonists and antagonists on smooth muscle and platelets. Br J Pharmacol 94:591-601.

Elberg G, Elberg D, Lewis TV, Guruswamy S, Chen L, Logan CJ, Chan MD, and Turman MA (2007) $\mathrm{EP}_{2}$ receptor mediates $\mathrm{PGE}_{2}$-induced cystogenesis of human renal epithelial cells. Am J Physiol Renal Physiol 293:F1622-F1632.

Enomoto Y, Adachi S, Matsushima-Nishiwaki R, Doi T, Niwa M, Akamatsu S, Tokuda H, Ogura S, Yoshimura S, Iwama T, et al. (2010) Thromboxane A promotes soluble CD40 ligand release from human platelets. Atherosclerosis 209: 415-421.

Fabre JE, Nguyen M, Athirakul K, Coggins K, McNeish JD, Austin S, Parise LK FitzGerald GA, Coffman TM, and Koller BH (2001) Activation of the murine EP receptor for $\mathrm{PGE}_{2}$ inhibits cAMP production and promotes platelet aggregation. J Clin Invest 107:603-610.

Faour WH, He Y, He QW, de Ladurantaye M, Quintero M, Mancini A, and Di Battista JA (2001) Prostaglandin $\mathrm{E}_{2}$ regulates the level and stability of cyclooxygenase-2 mRNA through activation of p38 mitogen-activated protein kinase in interleukin- $1 \beta$-treated human synovial fibroblasts. J Biol Chem 276:3172031731

Félétou M, Verbeuren TJ, and Vanhoutte PM (2009) Endothelium-dependent contractions in SHR: a tale of prostanoid TP and IP receptors. $\mathrm{Br} J$ Pharmacol 156:563-574.

Fernandes B and Crankshaw D (1995) ) Functional characterization of the prostanoid DP receptor in human myometrium. Eur $J$ Pharmacol 283:73-81.

Fetalvero KM, Martin KA, and Hwa J (2007) Cardioprotective prostacyclin signaling in vascular smooth muscle. Prostaglandins Other Lipid Mediat 82:109-118.

Fetalvero KM, Zhang P, Shyu M, Young BT, Hwa J, Young RC, and Martin KA (2008) Prostacyclin primes pregnant human myometrium for an enhanced contractile response in parturition. J Clin Invest 118:3966-3979.

Fiebich BL, Schleicher S, Spleiss O, Czygan M, and Hüll M (2001) Mechanisms of prostaglandin $\mathrm{E}_{2}$-induced interleukin- 6 release in astrocytes: possible involvement of $\mathrm{EP}_{4}$-like receptors, p38 mitogen-activated protein kinase and protein kinase $\mathrm{C}$ $J$ Neurochem 79:950-958.

Fischer DP, Hutchinson JA, Farrar D, O'Donovan PJ, Woodward DF, and Marshall $\mathrm{KM}$ (2008) Loss of prostaglandin $\mathrm{F}_{2 \alpha}$, but not thromboxane, responsiveness in pregnant human myometrium during labour. J Endocrinol 197:171-179.

Fleming EF, Athirakul K, Oliverio MI, Key M, Goulet J, Koller BH, and Coffman TM (1998) Urinary concentrating function in mice lacking $\mathrm{EP}_{3}$ receptors for prostaglandin $\mathrm{E}_{2}$. Am J Physiol 275:F955-F961.

Folger WH, Halushka PV, Wilcox CS, and Guzman NJ (1992) Characterization of rat glomerular thromboxane $\mathrm{A}_{2}$ receptors: comparison to rat platelets. Eur J Pharmacol 227:71-78.

Foord SM, Marks B, Stolz M, Bufflier E, Fraser NJ, and Lee MG (1996) The structure of the prostaglandin $\mathrm{EP}_{4}$ receptor gene and related pseudogenes. Genomics 35: $182-188$

Forrest AS, Hennig GW, Jokela-Willis S, Park CD, and Sanders KM (2009) Prostaglandin regulation of gastric slow waves and peristalsis. Am J Physiol Gastrointest Liver Physiol 296:G1180-G1190.

Fortner CN, Breyer RM, and Paul RJ (2001) $\mathrm{EP}_{2}$ receptors mediate airway relaxation to substance P, ATP, and $\mathrm{PGE}_{2}$. Am J Physiol Lung Cell Mol Physiol 281:L469-L474

Foudi N, Kotelevets L, Louedec L, Leséche G, Henin D, Chastre E, and Norel X (2008) Vasorelaxation induced by prostaglandin $\mathrm{E}_{2}$ in human pulmonary vein: role of the $\mathrm{EP}_{4}$ receptor subtype. Br J Pharmacol 154:1631-1639.

Francois H, Athirakul K, Howell D, Dash R, Mao L, Kim HS, Rockman HA, Fitzgerald GA, Koller BH, and Coffman TM (2005) Prostacyclin protects against elevated blood pressure and cardiac fibrosis. Cell Metab 2:201-207.

Francois H, Makhanova N, Ruiz P, Ellison J, Mao L, Rockman HA, and Coffman TM (2008) A role for the thromboxane receptor in L-NAME hypertension. Am J Physiol Renal Physiol 295:F1096-F1102.

Frias MA, Rebsamen MC, Gerber-Wicht C, and Lang U (2007) Prostaglandin E activates Stat3 in neonatal rat ventricular cardiomyocytes: a role in cardiac hypertrophy. Cardiovasc Res 73:57-65.

Friel AM, O'Reilly MW, Sexton DJ, and Morrison JJ (2005) Specific PGF ${ }_{2 \alpha}$ receptor (FP) antagonism and human uterine contractility in vitro. BJOG 112:1034-1042. Friis UG, Stubbe J, Uhrenholt TR, Svenningsen P, Nüsing RM, Skøtt O, and Jensen $\mathrm{BL}$ (2005) Prostaglandin $\mathrm{E}_{2} \mathrm{EP}_{2}$ and $\mathrm{EP}_{4}$ receptor activation mediates cAMPdependent hyperpolarization and exocytosis of renin in juxtaglomerular cells Am J Physiol Renal Physiol 289:F989-F997.

Fu LW, Phan A, and Longhurst JC (2008) Myocardial ischemia-mediated excitatory reflexes: a new function for thromboxane $\mathrm{A}_{2}$ ? Am J Physiol Heart Circ Physiol 295:H2530-H2540.

Fujimoto Y, Iwagaki H, Ozaki M, Ogino T, Murata H, Sun DS, Sadamori H, Takahashi HK, Tanaka N, and Yagi T (2005) Involvement of prostaglandin receptors (EPR2-4) in in vivo immunosuppression of $\mathrm{PGE}_{2}$ in rat skin transplant model. Int Immunopharmacol 5:1131-1139.

Fujino $\mathrm{H}$ and Regan JW (2006) $\mathrm{EP}_{4}$ prostanoid receptor coupling to a pertussis toxin-sensitive inhibitory G protein. Mol Pharmacol 69:5-10.

Fujino H, Salvi S, and Regan JW (2005) Differential regulation of phosphorylation of the cAMP element-binding protein after activation of $\mathrm{EP}_{2}$ and $\mathrm{EP}_{4}$ prostanoid receptors by prostaglandin $\mathrm{E}_{2}$. Mol Pharmacol 68:251-259.

Fujino H, West KA, and Regan JW (2002) Phosphorylation of glycogen synthase 
kinase-3 and stimulation of T-cell factor signaling following activation of $\mathrm{EP}_{2}$ and $\mathrm{EP}_{4}$ prostanoid receptors by prostaglandin $\mathrm{E}_{2}$. J Biol Chem 277:2614-2619.

Fujino H, Xu W, and Regan JW (2003) Prostaglandin $\mathrm{E}_{2}$ induced functional expression of early growth response factor- 1 by $\mathrm{EP}_{4}$, but not $\mathrm{EP}_{2}$, prostanoid receptors via the phosphatidylinositol 3-kinase and extracellular signal-regulated kinases. J Biol Chem 278:12151-12156.

Fujino T, Nakagawa N, Yuhki K, Hara A, Yamada T, Takayama K, Kuriyama S, Hosoki Y, Takahata O, Taniguchi T, et al. (2004) Decreased susceptibility to renovascular hypertension in mice lacking the prostaglandin $\mathrm{I}_{2}$ receptor IP. J Clin Invest 114:805-812.

Fujishima H, Fukagawa K, Okada N, Takano Y, Tsubota K, Hirai H, Nagata K, Matsumoto K, and Saito H (2005) Prostaglandin $\mathrm{D}_{2}$ induces chemotaxis in eosinophils via its receptor CRTH2 and eosinophils may cause severe ocular inflammation in patients with allergic conjunctivitis. Cornea 24:S66-S70.

Fukuda Y, Sugimura M, Suzuki K, and Kanayama N (2007) Prostaglandin E2 receptor EP4-selective antagonist inhibits lipopolysaccharide-induced cervical ripening in rabbits. Acta Obstet Gynecol Scand 86:1297-1302.

Fukushima M, Kato T, Ota K, Arai Y, Narumiya S, and Hayaishi O (1982) 9-Deoxydelta 9-prostaglandin $\mathrm{D}_{2}$, a prostaglandin $\mathrm{D}_{2}$ derivative with potent antineoplastic and weak smooth muscle-contracting activities. Biochem Biophys Res Commun 109:626-633.

Funk CD, Furci L, FitzGerald GA, Grygorczyk R, Rochette C, Bayne MA, Abramovitz M, Adam M, and Metters KM (1993) Cloning and expression of a cDNA for the human prostaglandin $\mathrm{E}$ receptor $\mathrm{EP}_{1}$ subtype. J Biol Chem 268:26767-26772.

Furci L, Fitzgerald DJ, and Fitzgerald GA (1991) Heterogeneity of prostaglandin $\mathrm{H}_{2}$ /thromboxane $\mathrm{A}_{2}$ receptors: distinct subtypes mediate vascular smooth muscle contraction and platelet aggregation. J Pharmacol Exp Ther 258:74-81.

Furuyashiki T and Narumiya S (2009) Roles of prostaglandin E receptors in stress responses. Curr Opin Pharmacol 9:31-38.

Gallant M, Carrière MC, Chateauneuf A, Denis D, Gareau Y, Godbout C, Greig G, Juteau H, Lachance N, Lacombe P, et al. (2002) Structure-activity relationship of biaryl acylsulfonamide analogues on the human $\mathrm{EP}_{3}$ prostanoid receptor. Bioorg Med Chem Lett 12:2583-2586.

Gallant MA, Samadfam R, Hackett JA, Antoniou J, Parent JL, and de BrumFernandes AJ (2005) Production by prostaglandin $\mathrm{D}_{2}$ by human osteoblasts and modulation of osteoprotegerin, RANKL, and cellular migration by DP and CRTH2 receptors. J Bone Miner Res 20:672-681.

Gallant MA, Slipetz D, Hamelin E, Rochdi MD, Talbot S, de Brum-Fernandes AJ, and Parent JL (2007) Differential regulation of the signaling and trafficking of the two prostaglandin D2 receptors, prostanoid DP receptor and CRTH2. Eur J Pharmacol 557:115-123.

Gao Q, Zhan P, Alander CB, Kream BE, Hao C, Breyer MD, Pilbeam CC, and Raisz LG (2009) Effects of global or targeted deletion of the EP4 receptor on the response of osteoblasts to prostaglandin in vitro and on bone histomorphometry in aged mice. Bone 45:98-103.

Gardiner PJ (1986) Characterization of prostanoid relaxant/inhibitory receptors (psi) using a highly selective agonist, TR4979. Br J Pharmacol 87:45-56.

Gaton DD, Sagara T, Lindsey JD, Gabelt BT, Kaufman PL, and Weinreb RN (2001) Increased matrix metalloproteinases 1,2 , and 3 in the monkey uveoscleral outflow pathway after topical prostaglandin $\mathrm{F}_{2 \alpha}$-isopropyl ester treatment. Arch Ophthalmol 119:1165-1170.

Gendron ME and Thorin E (2007) A change in the redox environment and thromboxane $\mathrm{A}_{2}$ production precede endothelial dysfunction in mice. Am J Physiol Heart Circ Physiol 293:H2508-H2515.

Genetos DC, Lee CM, Wong A, and Yellowley CE (2009) H1F-1alpha regulates hypoxia-induced $\mathrm{EP}_{1}$ expression in osteoblastic cells. J Cell Biochem 107:233-239.

George RJ, Sturmoski MA, Anant S, and Houchen CW (2007) EP4 mediates PGE dependent cell survival through PI3 kinase/AKT pathway. Prostaglandins Other Lipid Mediat 83:112-120.

Gerashchenko D, Beuckmann CT, Kanaoka Y, Eguchi N, Gordon WC, Urade Y, Bazan NG, and Hayaishi O (1998) Dominant expression of rat prostanoid DP receptor mRNA in leptomeninges, inner segments of photoreceptor cells, iris epithelium, and ciliary processes. $J$ Neurochem 71:937-945.

Gerlo S, Verdood P, Gellersen B, Hooghe-Peters EL, and Kooijman R (2004) Mechanism of prostaglandin $(\mathrm{PG}) \mathrm{E}_{2}$-induced prolactin expression in human $\mathrm{T}$ cells: cooperation of two $\mathrm{PGE}_{2}$ receptor subtypes, E-prostanoid (EP)3 and EP4, via calcium- and cyclic adenosine $5^{\prime}$-monophosphate-mediated signaling pathways. J Immunol 173:5952-5962.

Gervais FG, Cruz RP, Chateauneuf A, Gale S, Sawyer N, Nantel F, Metters KM, and O'Neill GP (2001) Selective modulation of chemokinesis, degranulation, and apoptosis in eosinophils through the $\mathrm{PGD}_{2}$ receptors CRTH2 and DP. J Allergy Clin Immunol 108:982-988.

Gervais FG, Morello JP, Beaulieu C, Sawyer N, Denis D, Greig G, Malebranche AD, and O'Neill GP (2005) Identification of a potent and selective synthetic agonist at the CRTH2 receptor. Mol Pharmacol 67:1834-1839.

Giblin GM, Bit RA, Brown SH, Chaignot HM, Chowdhury A, Chessell IP, Clayton NM, Coleman T, Hall A, Hammond B, et al. (2007) The discovery of 6-[2-(5-chloro2-\{[(2,4-difluorophenyl)methyl]oxy\}phenyl)-1-cyclopenten-1-yl]-2-pyridinecarboxylic acid, GW848687X, a potent and selective prostaglandin $\mathrm{EP}_{1}$ receptor antagonist for the treatment of inflammatory pain. Bioorg Med Chem Lett 17:385-389.

Giles H and Leff P (1988) The biology and pharmacology of $\mathrm{PGD}_{2}$. Prostaglandins 35:277-300.

Giles H, Leff P, Bolofo ML, Kelly MG, and Robertson AD (1989) The classification of prostaglandin DP-receptors in platelets and vasculature using BW A868C, a novel, selective, and potent competitive antagonist. Br J Pharmacol 96:291-300.

Goh Y and Nakajima M (1990) Stimulatory effects of prostaglandin $\mathrm{D}_{2}$ analogues on adenylate cyclase in rabbit iris-ciliary body membrane fractions. Exp Eye Res 51:585-590.

Goh Y, Nakajima M, Azuma I, and Hayaishi O (1988) Effects of prostaglandin $\mathrm{D}_{2}$ and its analogues on intraocular pressure in rabbits. Jpn $J$ Ophthalmol 32:471-480. González AA, Céspedes C, Villanueva S, Michea L, and Vio CP (2009) E prostanoid-1 receptor regulates renal medullary $\alpha \mathrm{ENaC}$ in rats infused with angiotensin II Biochem Biophys Res Commun 389:372-377.

Gorman RR, Bunting S, and Miller OV (1977) Modulation of human platelet adenylate cyclase by prostaglandin (PGX). Prostaglandins 13:377-388.

Gosset P, Pichavant M, Faveeuw C, Bureau F, Tonnel AB, and Trottein F (2005) Prostaglandin $\mathrm{D}_{2}$ affects the differentiation and functions of human dendritic cells impact on the T cell response. Eur J Immunol 35:1491-1500.

Goulet JL, Pace AJ, Key ML, Byrum RS, Nguyen M, Tilley SL, Morham SG, Langenbach R, Stock JL, McNeish JD, et al. (2004) E-prostanoid 3 receptor mediate the proinflammatory actions of prostaglandin $\mathrm{E}_{2}$ in acute cutaneou inflammation. J Immunol 173:1321-1326.

Graham DA and Rush JW (2009) Cyclooxygenase and thromboxane/prostaglandin receptor contribute to aortic endothelium-dependent dysfunction in aging female spontaneously hypertensive rats. J Appl Physiol 107:1059-1067.

Graham S, Gamie Z, Polyzois I, Narvani AA, Tzafetta K, Tsiridis E, Helioti M, Mantalaris A, and Tsiridis E (2009) Prostaglandin $\mathrm{EP}_{2}$ and $\mathrm{EP}_{4}$ receptor agonist in bone formation and bone healing: in vivo and in vitro evidence. Expert Opin Investig Drugs 18:746-766.

Gray T, Nettesheim P, Loftin C, Koo JS, Bonner J, Peddada S, and Langenbach R (2004) Interleukin-1 $\beta$-induced mucin production in human airway epithelium is mediated by cyclooxygenase-2, prostaglandin $\mathrm{E}_{2}$ receptors and cyclic AMP-protein kinase A signaling. Mol Pharmacol 66:337-346.

Gresele P, Blockmans D, Deckmyn H, and Vermylen J (1988) Adenylate cyclase activation determines the effect of thromboxane synthase inhibitors on platelet aggregation in vitro. Comparison of platelets from responders and nonresponders. J Pharmacol Exp Ther 246:301-307.

Griffin BW, Klimko P, Crider JY, and Sharif NA (1999) AL-8810: a novel prostaglandin F2 alpha analog with selective antagonist effects at the prostaglandin F2 alpha (FP) receptor. J Pharmacol Exp Ther 290:1278-1284.

Grigsby PL, Sooranna SR, Adu-Amankwa B, Pitzer B, Brockman DE, Johnson MR and Myatt L (2006) Regional expression of prostaglandin E2 and F2 $\alpha$ receptors in human myometrium, amnion, and choriodecidua with advancing gestation and labor. Biol Reprod 75:297-305.

Grimstrup M, Rist Ø, Receveur JM, Frimurer TM, Ulven T, Mathiesen JM, Kosteni E, and Högberg T (2010) Novel selective thiazoleacetic acids as CRTH2 antagonists developed from in silico derived hits. Part 2. Bioorg Med Chem Lett 20:11811185

Gross S, Tilly P, Hentsch D, Vonesch JL, and Fabre JE (2007) Vascular wallproduced prostaglandin $\mathrm{E}_{2}$ exacerbates arterial thrombosis and atherothrombosis through platelet $\mathrm{EP}_{3}$ receptors. J Exp Med 204:311-320

Gryglewski RJ (2008) Prostacyclin among prostanoids. Pharmacol Rep 60:3-11.

Guan Y, Zhang Y, Breyer RM, Fowler B, Davis L, Hébert RL, and Breyer MD (1998 Prostaglandin $\mathrm{E}_{2}$ inhibits renal collecting duct $\mathrm{Na}^{+}$absorption by activating the $\mathrm{EP}_{1}$ receptor. $J$ Clin Invest 102:194-201.

Guan Y, Zhang Y, Wu J, Qi Z, Yang G, Dou D, Gao Y, Chen L, Zhang X, Davis LS, et al. (2007) Antihypertensive effects of selective prostaglandin $\mathrm{E}_{2}$ receptor subtype 1 targeting. $J$ Clin Invest 117:2496-2505.

Guo N, Baglole CJ, O'Loughlin CW, Feldon SE, and Phipps RP (2010) Mast cellderived prostaglandin $\mathrm{D}_{2}$ controls hyaluronan synthesis in human orbital fibroblasts via $\mathrm{DP}_{1}$ activation: implications for thyroid eye disease. $J$ Biol Chem 285:15794-15804.

Gustafsson A, Hansson E, Kressner U, Nordgren S, Andersson M, Wang W, Lönnroth C, and Lundholm K (2007) $\mathrm{EP}_{1-4}$ subtype, COX and PPAR $\gamma$ receptor expression in colorectal cancer in prediction of disease-specific mortality. Int $J$ Cancer 121:232-240.

Gwóźdź P, Drelicharz L, Kozlovski VI, and Chlopicki S (2007) Prostacyclin, but not nitric oxide, is the major mediator of acetylcholine-induced vasodilatation in the isolated mouse heart. Pharmacol Rep 59:545-552.

Habib A, FitzGerald GA, and Maclouf J (1999) Phosphorylation of the thromboxane receptor $\alpha$, the predominant isoform expressed in human platelets. $J$ Biol Chem 274:2645-2651.

Hagino H, Kuraoka M, Kameyama Y, Okano T, and Teshima R (2005) Effect of a selective agonist for prostaglandin $\mathrm{E}$ receptor subtype $\mathrm{EP}_{4}$ (ONO-4819) on the cortical bone response to mechanical loading. Bone 36:444-453.

Hall A, Atkinson S, Brown SH, Chessell IP, Chowdhury A, Giblin GM, Goldsmith P, Healy MP, Jandu KS, Johnson MR, et al. (2007a) Discovery of novel, non-acidic 1,5-biaryl pyrrole EP1 receptor antagonists. Bioorg Med Chem Lett 17:1200-1205

Hall A, Billinton A, and Giblin GM (2007b) $\mathrm{EP}_{1}$ antagonists for the treatment of inflammatory pain. Curr Opin Drug Discov Devel 10:597-612.

Hall A, Brown SH, Chowdhury A, Giblin GM, Gibson M, Healy MP, Livermore DG Wilson RJ, Naylor A, Rawlings DA, et al. (2007c) Identification and optimization of novel 1,3,4-oxadiazole EP1 receptor antagonists. Bioorg Med Chem Lett 17: $4450-4455$.

Hallinan EA, Stapelfeld A, Savage MA, and Reichman M (1994) 8-Chlorodibenz[ $[b, f][1,4]$ oxazepine-10 $(11 H)$-carboxylic acid, 2-[3-[2-(furanylmethyl)thio]-1oxopropyl]hydrazide (SC-51322): a potent $\mathrm{PGE}_{2}$ antagonist and analgesic. Bioorg Med Chem Lett 4:509-514.

Halushka PV, Mais DE, Mayeux PR, and Morinelli TA (1989) Thromboxane, prostaglandin and leukotriene receptors. Annu Rev Pharmacol Toxicol 29:213-239.

Hammad H, Kool M, Soullié T, Narumiya S, Trottein F, Hoogsteden HC, and Lambrecht BN (2007) Activation of the D prostanoid 1 receptor suppresses asthma by modulation of lung dendritic cell function and induction of regulatory $\mathrm{T}$ cells. $J$ Exp Med 204:357-367.

Han C and Wu T (2005) Cycooxygenase-2-derived prostaglandin $\mathrm{E}_{2}$ promotes human cholangiocarcinoma cell growth and invasion through $\mathrm{EP}_{1}$ receptor-mediated activation of the epidermal growth factor receptor and Akt. J Biol Chem 280:2405324063.

Han S, Ritzenthaler JD, Wingerd B, Rivera HN, and Roman J (2007) Extracellular matrix fibronectin increases prostaglandin $\mathrm{E}_{2}$ receptor subtype $\mathrm{EP}_{4}$ in lung carcinoma cells through multiple signaling pathways: the role of AP-2. J Biol Chem 282:7961-7972. 
Han S and Roman J (2004) Suppression of prostaglandin $\mathrm{E}_{2}$ receptor subtype $\mathrm{EP}_{2}$ by PPARgamma ligands inhibits human lung carcinoma cell growth. Biochem Biophys Res Commun 314:1093-1099.

Hara A, Yuhki K, Fujino T, Yamada T, Takayama K, Kuriyama S, Takahata O, Karibe H, Okada Y, Xiao CY, et al. (2005) Augmented cardiac hypertrophy in response to pressure overload in mice lacking the prostaglandin $\mathrm{I}_{2}$ receptor. Circulation 112:84-92.

Harding P, Yang XP, Yang J, Shesely E, He Q, and LaPointe MC (2010) Gene expression profiling of dilated cardiomyopathy in older male EP4 knockout mice. Am J Physiol Heart Circ Physiol 298:H623-H632.

Harizi H, Grosset C, and Gualde N (2003) Prostaglandin $\mathrm{E}_{2}$ modulates dendritic cell function via $\mathrm{EP}_{2}$ and $\mathrm{EP}_{4}$ receptor subtypes. J Leukoc Biol 73:756-763.

Hartke JR and Lundy MW (2001) Bone anabolic therapy with selective prostaglandin analogs. J Musculoskelet Neuronal Interact 2:25-31.

Hartney JM, Coggins KG, Tilley SL, Jania LA, Lovgren AK, Audoly LP, and Koller $\mathrm{BH}$ (2006) Prostaglandin $\mathrm{E}_{2}$ protects lower airways against bronchoconstriction. Am J Physiol Lung Cell Mol Physiol 290:L105-L113.

Harvey RJ, Depner UB, Wässle H, Ahmadi S, Heindl C, Reinold H, Smart TG, Harvey K, Schütz B, Abo-Salem OM, et al. (2004) GlyR alpha3: an essential target for spinal $\mathrm{PGE}_{2}$-mediated inflammatory pain sensitization. Science 304:884-887.

Hase S, Yokota A, Nakagiri A, and Takeuchi K (2003) Prostaglandin $\mathrm{E}_{2}$ aggravates gastric mucosal injury induced by histamine in rats through $\mathrm{EP}_{1}$ receptors. Life Sci 74:629-641.

Hasegawa H, Katoh H, Yamaguchi Y, Nakamura K, Futakawa S, and Negishi M (2000) Different membrane targeting of prostaglandin $\mathrm{EP}_{3}$ receptor isoforms dependent on their carboxy-terminal tail structures. FEBS Lett 473:76-80.

Hasegawa H, Negishi M, and Ichikawa A (1996) Two isoforms of the prostaglandin $\mathrm{E}$ receptor $\mathrm{EP}_{3}$ subtype different in agonist-independent constitutive activity. $J$ Biol Chem 271:1857-1860

Hasegawa H, Negishi M, Katoh H, and Ichikawa A (1997) Two isoforms of prostaglandin $\mathrm{EP}_{3}$ receptor exhibiting constitutive activity and agonist-dependent activity in Rho-mediated stress fiber formation. Biochem Biophys Res Commun 234: $631-636$.

Hata AN and Breyer RM (2004) Pharmacology and signaling of prostaglandin receptors: multiple roles in inflammation and immune modulation. Pharmacol Ther 103:147-166.

Hata AN, Lybrand TP, and Breyer RM (2005) Identification of determinants of ligand binding affinity and selectivity in the prostaglandin $\mathrm{D}_{2}$ receptor CRTH2. J Biol Chem 280:32442-32451.

Hata AN, Zent R, Breyer MD, and Breyer RM (2003) Expression and molecular pharmacology of the mouse CRTH2 receptor. J Pharmacol Exp Ther 306:463-470.

Hatazawa R, Ohno R, Tanigami M, Tanaka A, and Takeuchi K (2006) Role of endogenous prostaglandins and cyclooxygenase isozymes in healing of indomethacin-induced small intestinal lesions in rats. J Pharmacol Exp Ther 318:691-699.

Hategan G, Polozov AM, Zeller W, Cao H, Mishra RK, Kiselyov AS, Ramirez J, Halldorsdottir G, Andrésson T, Gurney ME, et al. (2009) Heterocyclic 1,7disubstituted indole sulfonamides are potent and selective human $\mathrm{EP}_{3}$ receptor antagonists. Bioorg Med Chem Lett 19:6797-6800.

Hattori R, Shimizu S, Majima Y, and Shimizu T (2008) EP4 agonist inhibits lipopolysaccharide-induced mucus secretion in airway epithelial cells. Ann Otol Rhinol Laryngol 117:51-58.

Hawcroft G, Ko CW, and Hull MA (2007) Prostaglandin E2-EP4 receptor signalling promotes tumorigenic behaviour of HT-29 human colorectal cancer cells. Oncogene 26:3006-3019

Hayashi T, Nishihira J, Koyama Y, Sasaki S, and Yamamoto Y (2006) Decreased prostaglandin $\mathrm{E}_{2}$ production by inflammatory cytokine and lower expression of $\mathrm{EP}_{2}$ receptor result in increased collagen synthesis in keloid fibroblasts. J Invest Derm 126:990-997.

He Q, Harding P, and LaPointe MC (2010) PkA, Rap-1, ERK1/2, and p90RSK mediate PGE2 and EP4 signaling in neonatal ventricular myocytes. Am J Physiol Heart Circ Physiol 298:H136-H143.

Heavey DJ, Lumley P, Barrow SE, Murphy MB, Humphrey PP, and Dollery CT (1984) Effects of intravenous infusions of prostaglandin $\mathrm{D}_{2}$ in man. Prostaglandins 28:755-767.

Hébert RL, Carmosino M, Saito O, Yang G, Jackson CA, Qi Z, Breyer RM, Natarajan C, Hata AN, Zhang Y, et al. (2005) Characterization of a rabbit kidney prostaglandin $\mathrm{F}_{2 \alpha}$ receptor exhibiting $\mathrm{G}_{\mathrm{i}}$-restricted signaling that inhibits water absorption in the collecting duct. $J$ Biol Chem 280:35028-35037.

Hébert RL, O'Connor T, Neville C, Burns KD, Laneuville O, and Peterson LN (1998) Prostanoid signaling, localization, and expression of IP receptors in rat thick ascending limb cells. Am J Physiol 275:F904-F914.

Hedberg A, Hall SE, Ogletree ML, Harris DN, and Liu EC (1988) Characterization of $\left[5,6-{ }^{3} \mathrm{H}\right] \mathrm{SQ} 29,548$ as a high affinity radioligand, binding to thromboxane $\mathrm{A}_{2}$ prostaglandin $\mathrm{H}_{2}$-receptors in human platelets. J Pharmacol Exp Ther 245:786792.

Hellberg MR, Sallee VL, McLaughlin MA, Sharif NA, Desantis L, Dean TR, and Zinke PW (2001) Preclinical efficacy of travoprost, a potent and selective FP prostaglandin receptor agonist. J Ocul Pharmacol Ther 17:421-432.

Heptinstall S, Espinosa DI, Manolopoulos P, Glenn JR, White AE, Johnson A, Dovlatova N, Fox SC, May JA, Hermann D, et al. (2008) DG-041 inhibits the EP3 prostanoid receptor-a new target for inhibition of platelet function in atherothrombotic disease. Platelets 19:605-613.

Herlong JL and Scott TR (2006) Positioning prostanoids of the D and J series in the immunopathogenic scheme. Immunol Lett 102:121-131.

Hervé M, Angeli V, Pinzar E, Wintjens R, Faveeuw C, Narumiya S, Capron A, Urade Y, Capron M, Riveau G, et al. (2003) Pivotal roles of the parasite PGD $_{2}$ synthase and of the host D prostanoid receptor 1 in schistosome immune evasion. Eur J Immunol 33:2764-2772.

Hildebrandt JD (2006) Bring your own G protein. Mol Pharmacol 69:1079-1082

Hirai H, Tanaka K, Takano S, Ichimasa M, Nakamura M, and Nagata K (2002)
Cutting edge: agonist effect of indomethacin on a prostaglandin $\mathrm{D}_{2}$ receptor, CRTH2. J Immunol 168:981-985.

Hirai H, Tanaka K, Yoshie O, Ogawa K, Kenmotsu K, Takamori Y, Ichimasa M, Sugamura K, Nakamura M, Takano S, et al. (2001) Prostaglandin $\mathrm{D}_{2}$ selectively induces chemotaxis in T helper type 2 cells, eosinophils, and basophils via seven transmembrane receptor CRTH2. J Exp Med 193:255-261.

Hirata M, Hayashi Y, Ushikubi F, Yokota Y, Kageyama R, Nakanishi S, and Narumiya S (1991) Cloning and expression of cDNA for a human thromboxane $A_{2}$ receptor. Nature 349:617-620

Hirata M, Kakizuka A, Aizawa M, Ushikubi F, and Narumiya S (1994) Molecular characterization of a mouse prostaglandin D receptor and functional expression of the cloned gene. Proc Natl Acad Sci USA 91:11192-11196.

Hirata T, Ushikubi F, Kakizuka A, Okuma M, and Narumiya S (1996) Two thromboxane $\mathrm{A}_{2}$ receptor isoforms in human platelets. Opposite coupling to adenylyl cyclase with different sensitivity to Arg60 to Leu mutation. J Clin Invest 97:949956.

Hizaki H, Hasegawa H, Katoh H, Negishi M, and Ichikawa A (1997) Functional role of carboxyl-terminal tail of prostaglandin $\mathrm{EP}_{3}$ receptor in $\mathrm{G}_{\mathrm{i}}$ coupling. FEBS Let 414:323-326.

Hizaki H, Segi E, Sugimoto Y, Hirose M, Saji T, Ushikubi F, Matsuoka T, Noda Y, Tanaka T, Yoshida N, et al. (1999) Abortive expansion of the cumulus and impaired fertility in mice lacking the prostaglandin E receptor subtype $\mathrm{EP}(2)$. Proc Natl Acad Sci USA 96:10501-10506.

Hoang B, Trinh A, Birnbaumer L, and Edwards RA (2007) Decreased MAPK-and $\mathrm{PGE}_{2}$-dependent IL-11 production in $\mathrm{G}_{\mathrm{i} \alpha 2}-/-$ colonic fibroblasts. Am J Physio Gastrointest Liver Physiol 292:G1511-G1519.

Hoet B, Falcon C, De Reys S, Arnout J, Deckmyn H, and Vermylen J (1990) R68070 a combined thromboxane/endoperoxide receptor antagonist and thromboxane synthase inhibitor, inhibits human platelet activation in vitro and in vivo: a comparison with aspirin. Blood 75:646-653.

Hofstetter AO, Saha S, Siljehav V, Jakobsson PJ, and Herlenius E (2007) The induced prostaglandin $\mathrm{E}_{2}$ pathway is a key regulator of the respiratory response to infection and hypoxia in neonates. Proc Natl Acad Sci USA 104:9894-9899.

Hohlfeld T, Meyer-Kirchrath J, Vogel YC, and Schrör K (2000) Reduction of infarct size by selective stimulation of prostaglandin $\mathrm{EP}(3)$ receptors in the reperfused ischemic pig heart. J Mol Cell Cardiol 32:285-296.

Hohlfeld T, Zucker TP, Meyer J, and Schrör K (1997) Expression, function, and regulation of E-type prostaglandin receptors (EP3) in the nonischemic and ischemic pig heart. Circ Res 81:765-773.

Holz GG, Kang G, Harbeck M, Roe MW, and Chepurny OG (2006) Cell physiology of cAMP sensor Epac. J Physiol 577:5-15.

Honda A, Sugimoto Y, Namba T, Watabe A, Irie A, Negishi M, Narumiya S, and Ichikawa A (1993) Cloning and expression of a cDNA for mouse prostaglandin E receptors $\mathrm{EP}_{2}$ subtype. J Biol Chem 268:7759-7762.

Honda K, Arima M, Cheng G, Taki S, Hirata H, Eda F, Fukushima F, Yamaguchi B Hatano M, Tokuhisa T, et al. (2003) Prostaglandin $\mathrm{D}_{2}$ reinforces Th2 type inflammatory responses of airways to low-dose antigen through bronchial expression of macrophage-derived chemokine. J Exp Med 198:533-543.

Honda T, Segi-Nishida E, Miyachi Y, and Narumiya S (2006) Prostacyclin-IP signaling and prostaglandin $\mathrm{E}_{2}-\mathrm{EP}_{2} / \mathrm{EP}_{4}$ signaling both mediate joint inflammation in mouse collagen-induced arthritis I Exp Med 203:325-335.

Honma S, Saika M, Ohkubo S, Kurose H, and Nakahata N (2006) Thromboxane A receptor-mediated $\mathrm{G}_{12} /{ }_{13}$-dependent glial morphological change. Eur J Pharmacol 545:100-108

Honma Y, Arai I, Hashimoto Y, Futaki N, Sugimoto M, Tanaka M, and Nakaike S (2005) Prostaglandin $\mathrm{D}_{2}$ and prostaglandin $\mathrm{E}_{2}$ accelerate the recovery of cutaneous barrier disruption induced by mechanical scratching in mice. Eur J Pharmacol 518:56-62.

Hori R, Nakagawa T, Sugimoto Y, Sakamoto T, Yamamoto N, Hamaguchi K, and Ito J (2009) Prostaglandin E receptor subtype EP4 agonist protects cochleae against noise-induced trauma. Neuroscience 160:813-819.

Hornberger W and Patscheke H (1989) Transient concentrations and agonist potency of $\mathrm{PGH}_{2}$ in platelet activation by endogenous arachidonate. Eicosanoids 2:241248

Horsley V and Pavlath GK (2003) Prostaglandin $\mathrm{F}_{2 \alpha}$ stimulates growth of skeletal muscle cells via an NFATC2-dependent pathway. J Cell Biol 161:111-118.

Hoshikawa H, Goto R, Mori T, Mitani T, and Mori N (2009) Expression of prostaglandin E2 receptors in oral squamous cell carcinomas and growth inhibitory effects of an EP3 selective antagonist, ONO-AE3-240. Int $J$ Oncol 34:847-852.

Hoshino T, Nakaya T, Homan T, Tanaka K, Sugimoto Y, Araki W, Narita M, Narumiya S, Suzuki T, and Mizushima T (2007) Involvement of prostaglandin $\mathrm{E}_{2}$ in production of amyloid-beta peptides both in vitro and in vivo. $J$ Biol Chem 282:32676-32688

Hoshino T, Namba T, Takehara M, Nakaya T, Sugimoto Y, Araki W, Narumiya S, Suzuki T, and Mizushima T (2009) Prostaglandin $\mathrm{E}_{2}$ stimulates the production of amyloid- $\beta$ peptides through internalization of the $\mathrm{EP}_{4}$ receptor. $J$ Biol Chem 284:18493-18502.

Hoshino T, Tsutsumi S, Tomisato W, Hwang HJ, Tsuchiya T, and Mizushima T (2003) Prostaglandin $\mathrm{E}_{2}$ protects gastric mucosal cells from apoptosis via $\mathrm{EP}_{2}$ and $\mathrm{EP}_{4}$ receptor activation. J Biol Chem 278:12752-12858.

Hosoi M, Oka T, Abe M, Hori T, Yamamoto H, Mine K, and Kubo C (1999) Prostaglandin $\mathrm{E}(2)$ has antinociceptive effect through $\mathrm{EP}(1)$ receptor in the ventromedial hypothalamus in rats. Pain 83:221-227.

Hosoi M, Oka T, and Hori T (1997) Prostaglandin E receptor $\mathrm{EP}_{3}$ subtype is involved in thermal hyperalgesia through its actions in the preoptic hypothalamus and the diagonal band of Broca in rats. Pain 71:303-311.

Hristovska AM, Rasmussen LE, Hansen PB, Nielsen SS, Nüsing RM, Narumiya S, Vanhoutte P, Skøtt O, and Jensen BL (2007) Prostaglandin $\mathrm{E}_{2}$ induces vascular relaxation by E-prostanoid 4 receptor-mediated activation of endothelial nitric oxide synthase. Hypertension 50:525-530.

Hsu PY, Tsai AL, Kulmacz RJ, and Wang LH (1999) Expression, purification, and 
spectroscopic characterization of human thromboxane synthase. $J$ Biol Chem 274:762-769.

Huang JC, Wun WS, Goldsby JS, Egan K, FitzGerald GA, and Wu KK (2007a) Prostacyclin receptor signaling and early embryo development in the mouse. Hum Reprod 22:2851-2856.

Huang JS, Ramamurthy SK, Lin X, and Le Breton GC (2004) Cell signalling through thromboxane $\mathrm{A}_{2}$ receptors. Cell Signal 16:521-533.

Huang S, Wettlaufer SH, Hogaboam C, Aronoff DM, and Peters-Golden M (2007b) Prostaglandin $\mathrm{E}_{2}$ inhibits collagen expression and proliferation in patient-derived normal lung fibroblasts via $\mathrm{E}$ prostanoid 2 receptor and cAMP signaling. Am J Physiol Lung Cell Mol Physiol 292:L405-L413.

Hung CH, Chu YT, Suen JL, Lee MS, Chang HW, Lo YC, and Jong YJ (2009) Regulation of cytokine expression in human plasmacytoid dendritic cells by prostaglandin $\mathrm{I}_{2}$ analogues. Eur Respir $J$ 33:405-410.

Hung GH, Jones RL, Lam FF, Chan KM, Hidaka H, Suzuki M, and Sasaki Y (2006) Investigation of the pronounced synergism between prostaglandin $\mathrm{E}_{2}$ and other constrictor agents on rat femoral artery. Prostaglandins Leukot Essent Fatty Acids 74:401-415.

Husain S, Jafri F, and Crosson CE (2005) Acute effects of $\mathrm{PGF}_{2 \alpha}$ on MMP-2 secretion from human ciliary muscle cells: a PKC-and ERK-dependent process. Invest Ophthalmol Vis Sci 46:1706-1713.

Hutchinson AJ, Chou CL, Israel DD, Xu W, and Regan JW (2009) Activation of $\mathrm{EP}_{2}$ prostanoid receptors in human glial cell lines stimulates the secretion of BDNF. Neurochem Int 54:439-446.

Hutchinson AJ, Coons SC, Chou CL, Xu W, Stamer WD, Woodward DF, and Regan JW (2010) Induction of angiogenic immediate early genes by activation of FP prostanoid receptors in cultured human ciliary smooth muscle cells. Curr Eye Res 35:408-418.

Hutchinson J, Marshall KM, and Senior J (2003) Preliminary studies using a putative FP-receptor antagonist, Al-8810, on isolated mouse uterus. Proceedings of the British Pharmacological Society. Available at: http://www.pa2online.org/ Vol1Issue3abst038P.html.

Ikeda M, Kawatani M, Maruyama T, and Ishihama H (2006) Prostaglandin facilitates afferent nerve activity via $\mathrm{EP}_{1}$ receptors during urinary bladder inflammation in rats. Biomed Res 27:49-54

Ikeda-Matsuo Y, Tanji H, Ota A, Hirayama Y, Uematsu S, Akira S, and Sasaki Y (2010) Microsomal prostaglandin E synthase-1 contributes to ischaemic excitotoxicity through prostaglandin $\mathrm{E}_{2} \mathrm{EP}_{3}$ receptors. $\mathrm{Br}$ J Pharmacol 160:847-859.

Imig JD, Breyer MD, and Breyer RM (2002) Contribution of prostaglandin EP(2) receptors to renal microvascular reactivity in mice. Am J Physiol Renal Physiol 283:F415-F422.

Imura Y, Terashita Z, Shibouta Y, Inada Y, and Nishikawa K (1990) Antagonistic action of AA-2414 on thromboxane $\mathrm{A}_{2}$ /prostaglandin endoperoxide receptor in platelets and blood vessels. Jpn J Pharmacol 52:35-43.

Imura Y, Terashita Z, Shibouta Y, and Nishikawa K (1988) The thromboxane $\mathrm{A}_{2} /$ prostaglandin endoperoxide receptor antagonist activity of CV-1451, a thromboxane $\mathrm{A}_{2}$ synthetase inhibitor. Eur $J$ Pharmacol 147:359-365.

Irie A, Sugimoto Y, Namba T, Asano T, Ichikawa A, and Negishi M (1994) The C-terminus of prostaglandin-E-receptor $\mathrm{EP}_{3}$ subtype is essential for activation of GTP-binding protein. Eur J Biochem 224:161-166.

Ishimura M, Maeda T, Kataoka S, Suda M, Kurokawa S, and Hiyama Y (2009) Effects of KP-496, a novel dual antagonist for cysteinyl leukotriene receptor 1 and thromboxane $\mathrm{A}_{2}$ receptor, on Sephadex-induced airway inflammation in rats. Biol Pharm Bull 32:1057-1061.

Ishiura Y, Fujimura M, Yamamori C, Nobata K, Myou S, Kurashima K, and Takegoshi T (2003) Thromboxane antagonism and cough in chronic bronchitis. Ann Med 35:135-139.

Ishizuka T, Kawakami M, Hidaka T, Matsuki Y, Takamizawa M, Suzuki K, Kurita $\mathrm{A}$, and Nakamura $\mathrm{H}$ (1998) Stimulation with thromboxane $\mathrm{A}_{2}\left(\mathrm{TXA}_{2}\right)$ receptor agonist enhances ICAM-1, VCAM-1 or ELAM-1 expression by human vascular endothelial cells. Clin Exp Immunol 112:464-470.

Ito S, Sakamoto K, Mochizuki-Oda N, Ezashi T, Miwa K, Okuda-Ashitaka E, Shevchenko VI, Kiso Y, and Hayaishi O (1994) Prostaglandin $\mathrm{F}_{2 \alpha}$ receptor is coupled to $\mathrm{G}_{\mathrm{q}}$ in cDNA-transfected Chinese hamster ovary cells. Biochem Biophys Res Commun 200:756-762.

Jabbour HN, Sales KJ, Boddy SC, Anderson RA, and Williams AR (2005) A positive feedback loop that regulates cyclooxygenase-2 expression and prostaglandin $\mathrm{F}_{2 a}$ synthesis via the F-series-prostanoid receptor and extracellular signal-regulated kinase 1/2 signaling pathway. Endocrinology 146:4657-4664.

Jadhav V, Jabre A, Chen MF, and Lee TJ (2009) Presynaptic prostaglandin $\mathrm{E}_{2}$ $\mathrm{EP}_{1}$-receptor facilitation of cerebral nitrergic neurogenic vasodilation. Stroke $\mathbf{4 0}$ 261-269.

Jaffar Z, Ferrini ME, Buford MC, Fitzgerald GA, and Roberts K (2007) Prostaglandin $\mathrm{I}_{2}$-IP signaling blocks allergic pulmonary inflammation by preventing recruitment of $\mathrm{CD}^{+}$Th2 cells into the airways in a mouse model of asthma. $J$ Immunol 179:6193-6203.

Jain S, Chakraborty G, Raja R, Kale S, and Kundu GC (2008) Prostaglandin E regulates tumor angiogenesis in prostate cancer. Cancer Res 68:7750-7759.

Jenkins DW, Feniuk W, and Humphrey PP (2001) Characterization of the prostanoid receptor types involved in mediating calcitonin gene-related peptide release from cultured rat trigeminal neurones. Br J Pharmacol 134:1296-1302.

Ji R, Chou CL, Xu W, Chen XB, Woodward DF, and Regan JW (2010) EP1 prostanoid receptor coupling to Gi/o up-regulates the expression of hypoxia-inducible factor- $1 \alpha$ through activation of a phosphoinositide-3 kinase signaling pathway. Mol Pharmacol 77:1025-1036.

Jiang GL, Nieves A, Im WB, Old DW, Dinh DT, and Wheeler L (2007) The prevention of colitis by $\mathrm{E}$ prostanoid receptor 4 agonist through enhancement of epithelium survival and regeneration. J Pharmacol Exp Ther 320:22-28.

Jin J, Mao GF, and Ashby B (1997) Constitutive activity of human prostaglandin E receptor $\mathrm{EP}_{3}$ isoforms. $\mathrm{Br} \mathrm{J}$ Pharmacol 121:317-323.

Jin J, Shie FS, Liu J, Wang Y, Davis J, Schantz AM, Montine KS, Montine TJ, and
Zhang J (2007) Prostaglandin $\mathrm{E}_{2}$ receptor subtype $2\left(\mathrm{EP}_{2}\right)$ regulates microglial activation and associated neurotoxicity induced by aggregated alpha-synuclein. $J$ Neuroinflammation 4:2.

Johnson MR, Schaaf TK, Constantine JW, and Hess HJ (1980) Structure activity studies leading to a tissue-selective hypotensive prostaglandin analog, 13,14 dihydro-16-phenyl-omega-tetranor PGE2. Prostaglandins 20:515-520.

Johnstone MA and Albert DM (2002) Prostaglandin-induced hair growth. Suru Ophthalmol 47:S185-S202.

Jones JH, Holtz WJ, Bicking JB, and Cragoe EJ Jr (1977) 11,12-Secoprostaglandins 4. 7-( $N$-alkylmethanesulfonamido) heptanoic acids. J Med Chem 20:1299-1304

Jones RL (1976a) Cardiovascular actions of prostaglandins D and E in the sheep evidence for two distinct receptors. Adv Prostaglandin Thromboxane Res 1:221230

Jones RL (1976b) Sites of action of prostaglandins on the cardiovascular system with particular reference to the sheep. Acta Biol Med Ger 35:1091-1096.

Jones RL (1978) Definition of prostaglandin-sensitive arterial constrictor systems. Acta Biol Med Ger 37:837-844.

Jones RL and Chan K (2001) Distinction between relaxations induced via prostanoid $\mathrm{EP}_{4}$ and $\mathrm{IP}_{1}$ receptors in pig and rabbit blood vessels. $\mathrm{Br} \mathrm{J}$ Pharmacol 134:313324 .

Jones RL and Chan KM (2005) Investigation of the agonist activity of prostacyclin analogues on prostanoid $\mathrm{EP}_{4}$ receptors using $\mathrm{GW} 627368$ and taprostene: evidence for species differences. Prostaglandins Leukot Essent Fatty Acids 72:289-299.

Jones RL, Giembycz MA, and Woodward DF (2009) Prostanoid receptor antagonists: development strategies and therapeutic applications. Br J Pharmacol 158:104145 .

Jones RL, Peesapati V, and Wilson NH (1982) Antagonism of the thromboxanesensitive contractile systems of the rabbit aorta, dog saphenous vein and guineapig trachea. Br J Pharmacol 76:423-438.

Jones RL, Qian YM, Chan KM, and Yim AP (1998) Characterization of a prostanoid $\mathrm{EP}_{3}$-receptor in guinea-pig aorta: partial agonist action of the non-prostanoid ONO-AP-324. Br J Pharmacol 125:1288-1296.

Jones RL, Qian YM, Wise H, Wong HN, Lam WL, Chan HW, Yim AP, and Ho JK (1997) Relaxant actions of nonprostanoid prostacyclin mimetics on human pulmonary artery. J Cardiovasc Pharmacol 29:525-535.

Jones RL and Wilson NH (1978) Tautomerism of the enedione system of 15oxoprostaglandin $\mathrm{D}_{2}$. J Chem Soc Perkin Trans 1 1978:209-214.

Jones RL and Wilson NH (1980) Partial agonism of prostaglandin $\mathrm{H}_{2}$ analogs and 11-deoxy-prostaglandin $\mathrm{F}_{2 \alpha}$ to thromboxane-sensitive preparations. Adv Prostaglandin Thromboxane Res 6:467-475.

Jones RL, Wilson NH, and Lawrence RA (1989) EP 171: a high affinity thromboxane $\mathrm{A}_{2}$-mimetic, the actions of which are slowly reversed by receptor blockade. $\mathrm{Br} J$ Pharmacol 96:875-887.

Jones RL, Wilson NH, Marr CG, Muir G, and Armstrong RA (1993) Diphenylmethylazine prostanoids with prostacyclin-like actions on human platelets. J Lipid Mediat 6:405-410.

Jones RL, Wise H, Clark R, Whiting RL, and Bley KR (2006) Investigation of the prostacyclin (IP) receptor antagonist RO1138452 on isolated blood vessel and platelet preparations. Br J Pharmacol 149:110-120.

Jones RL, Woodward DF, and Wang JW (2008) Investigation of the slow kinetics of the prostanoid $\mathrm{EP}_{3}$ receptor antagonists L-798106 and L-826266 on guinea-pig aorta (Abstract). Fundam Clin Pharmacol 22 (Suppl s2):78.

Jones RL, Woodward DF, Wang JW, and Clark RL (2011) Roles of affinity and lipophilicity in the slow kinetics of prostanoid receptor antagonists on isolated smooth muscle preparations. Br J Pharmacol 162:863-879.

Jovanović N, Pavlović M, Mircevski V, Du Q, and Jovanović A (2006) An unexpected negative inotropic effect of prostaglandin $\mathrm{F}_{2 \alpha}$ in the rat heart. Prostaglandins Other Lipid Mediat 80:110-119.

Kabashima K, Murata T, Tanaka H, Matsuoka T, Sakata D, Yoshida N, Katagiri K, Kinashi T, Tanaka T, Miyasaka M, et al. (2003a) Thromboxane A, modulates interaction of dendritic cells and $\mathrm{T}$ cells and regulates acquired immunity. Nat Immunol 4:694-701.

Kabashima K, Nagamachi M, Honda T, Nishigori C, Miyachi Y, Tokura Y, and Narumiya $\mathrm{S}$ (2007) Prostaglandin $\mathrm{E}_{2}$ is required for ultraviolet B-induced skin inflammation via EP2 and EP4 receptors. Lab Invest 87:49-55.

Kabashima K, Saji T, Murata T, Nagamachi M, Matsuoka T, Segi E, Tsuboi K, Sugimoto Y, Kobayashi T, Miyachi Y, et al. (2002) The prostaglandin receptor $\mathrm{EP}_{4}$ suppresses colitis, mucosal damage and CD4 cell activation in the gut. $J$ Clin Invest 109:883-893.

Kabashima K, Sakata D, Nagamachi M, Miyachi Y, Inaba K, and Narumiya S (2003b) Prostaglandin $\mathrm{E}_{2}-\mathrm{EP}_{4}$ signaling initiates skin immune responses by promoting migration and maturation of Langerhans cells. Nature Med 9:744-749.

Kamio K, Liu X, Sugiura H, Togo S, Kobayashi T, Kawasaki S, Wang X, Mao L, Ahn Y, Hogaboam C, et al. (2007) Prostacyclin analogs inhibit fibroblast contraction of collagen gels through the cAMP-PKA pathway. Am J Respir Cell Mol Biol 37:113120 .

Kamiyama M, Pozzi A, Yang L, DeBusk LM, Breyer RM, and Lin PC (2006) $\mathrm{EP}_{2}$, a receptor for $\mathrm{PGE}_{2}$, regulates tumor angiogenesis through direct effects on endothelial cell motility and survival. Oncogene 25:7019-7028.

Kamon M, Fujita D, Goto N, Amano H, and Sakamoto K (2008) Prostaglandin $\mathrm{F}_{2 a}$ negatively regulates bone resorption in murine osteoclast development. Prostaglandins Other Lipid Mediat 87:26-33.

Kamoshita E, Ikeda Y, Fujita M, Amano H, Oikawa A, Suzuki T, Ogawa Y, Yamashina S, Azuma S, Narumiya S, et al. (2006) Recruitment of a prostaglandin E receptor subtype, EP3-expressing bone marrow cells in crucial in wound-induced angiogenesis. Am J Pathol 169:1458-1472.

Kan KK, Ngan MP, Wai MK, and Rudd JA (2008) Mechanism of the prostanoid TP receptor agonist U46619 for inducing emesis in the ferret. Naunyn Schmiedebergs Arch Pharmacol 378:655-661.

Kanda N, Koike S, and Watanabe S (2005) Prostaglandin $E_{2}$ enhances neutrophin-4 
production via EP3 receptor in human keratinocytes. J Pharmacol Exp Ther 315:796-804.

Kassuya CA, Ferreira J, Claudino RF, and Calixto JB (2007) Intraplantar PGE causes nociceptive behaviour and mechanical allodynia: the role of prostaglandin E receptors and protein kinases. Br J Pharmacol 150:727-737.

Katagiri H, Ito Y, Ito S, Murata T, Yukihiko S, Narumiya S, Watanabe M, and Majima M (2008) TNF- $\alpha$ induces thromboxane receptor signaling-dependent microcirculatory dysfunction in mouse liver. Shock 30:463-467.

Kato S, Aihara E, Yoshii K, and Takeuchi K (2005) Dual action of prostaglandin E2 on gastric acid secretion through different EP-receptor subtypes in the rat. Am J Physiol Gastrointest Liver Physiol 289:G64-G69.

Katoh H, Negishi M, and Ichikawa A (1996) Prostaglandin E receptor $\mathrm{EP}_{3}$ subtype induces neurite retraction via small GTPase Rho. J Biol Chem 271:29780-29784.

Katoh H, Watabe A, Sugimoto Y, Ichikawa A, and Negishi M (1995) Characterization of the signal transduction of prostaglandin $\mathrm{E}$ receptor $\mathrm{EP}_{1}$ subytpe in cDNAtransfected Chinese hamster ovary cells. Biochim Biophys Acta 1244:41-48.

Katsura M, Miyamoto T, Hamanaka N, Kondo K, Terada T, Ohgaki Y, Kawasaki A, and Tsuboshima M (1983) In vitro and in vivo effects of new powerful thromboxane antagonists (3-alkylamino pinane derivatives). Adv Prostaglandin Thromboxane Leukot Res 11:351-357.

Kawamori T, Kitamura T, Watanabe K, Uchiya N, Maruyama T, Narumiya S, Sugimura T, and Wakabayashi K (2005) Prostaglandin E receptor subtype $\mathrm{EP}_{1}$ deficiency inhibits colon cancer development. Carcinogenesis 26:353-357.

Kawamori T, Uchiya N, Kitamura T, Ohuchida S, Yamamoto H, Maruyama T, Sugimura T, and Wakabayashi K (2001a) Evaluation of a selective prostaglandin $\mathrm{E}$ receptor $\mathrm{EP}_{1}$ antagonist for potential properties in colon carcinogenesis. Anticancer Res 21:3865-3869.

Kawamori T, Uchiya N, Nakatsugi S, Watanabe K, Ohuchida S, Yamamoto H, Maruyama T, Kondo K, Sugimura T, and Wakabayashi K (2001b) Chemopreventive effects of ONO-8711, a selective prostaglandin E receptor EP(1) antagonist, on breast cancer development. Carcinogenesis 22:2001-2004.

Kawano T, Anrather J, Zhou P, Park L, Wang G, Frys KA, Kunz A, Cho S, Orio M, and Iadecola $\mathrm{C}$ (2006) Prostaglandin $\mathrm{E}_{2} \mathrm{EP}_{1}$ receptors: downstream effectors of COX-2 neurotoxicity. Nat Med 12:225-229.

Kay LJ, Yeo WW, and Peachell PT (2006) Prostaglandin $\mathrm{E}_{2}$ activates $\mathrm{EP}_{2}$ receptors to inhibit human lung mast cell degranulation. Br J Pharmacol 147:707-713.

Ke HZ, Crawford DT, Qi H, Simmons HA, Owen TA, Paralkar VM, Li M, Lu B, Grasser WA, Cameron KO, et al. (2006) A nonprostanoid $\mathrm{EP}_{4}$ receptor selective prostaglandin $\mathrm{E}_{2}$ agonist restores bone mass and strength in aged, ovariectomized rats. J Bone Miner Res 21:565-575.

Keene CD, Chang R, Stephen C, Nivison M, Nutt SE, Look A, Breyer RM, Horner PJ, Hevner R, and Montine TJ (2009) Production of hippocampal neurogenesis from toll-like receptor 4-dependent innate immune activation by ablation of prostaglandin $\mathrm{E}_{2}$ receptor subtype $\mathrm{EP}_{1}$ or $\mathrm{EP}_{2}$. Am J Pathol 174:2300-2309.

Keery RJ and Lumley P (1988) AH6809, a prostaglandin DP-receptor blocking drug on human platelets. $\mathrm{Br}$ J Pharmacol 94:745-754.

Kelley-Hickie LP and Kinsella BT (2004) $\mathrm{EP}_{1^{-}}$and FP-mediated cross-desensitization of the alpha $(\alpha)$ and beta $(\beta)$ isoforms of the human thromboxane $\mathrm{A}_{2}$ receptor. Br J Pharmacol 142:203-221.

Kennedy CR, Xiong H, Rahal S, Vanderluit J, Slack RS, Zhang Y, Guan Y, Breyer $\mathrm{MD}$, and Hébert RL (2007) Urine concentrating defect in prostaglandin $\mathrm{EP}_{1^{-}}$ deficient mice. Am J Physiol Renal Physiol 292:F868-F875.

Kennedy CR, Zhang Y, Brandon S, Guan Y, Coffee K, Funk CD, Magnuson MA, Oates JA, Breyer MD, and Breyer RM (1999) Salt-sensitive hypertension and reduced fertility in mice lacking the prostaglandin $\mathrm{EP}_{2}$ receptor. Nature Med 5:217-220.

Kennedy I, Coleman RA, Humphrey PP, Levy GP, and Lumley P (1982) Studies on the characterisation of prostanoid receptors: a proposed classification. Prostaglandins 24:667-689.

Khera M, Boone TB, Salas N, Jett MF, and Somogyi GT (2007) The role of the prostacyclin receptor antagonist RO3244019 in treating neurogenic detrusor overactivity after spinal cord injury in rats. BJU Int 99:442-446.

Kim HJ, Chung JI, Lee SH, Jung YS, Moon CH, and Baik EJ (2008) Involvement of endogenous prostaglandin $\mathrm{F}_{2 \alpha}$ on kainic acid-induced seizure activity through FP receptor: the mechanism of proconvulsant effects of COX-2 inhibitors. Brain Res 1193:153-161.

Kim JI, Lakshmikanthan V, Frilot N, and Daaka Y (2010) Prostaglandin $E_{2}$ promotes lung cancer cell migration via $\mathrm{EP}_{4}$-betaArrestin1-c-Src signalsome. $\mathrm{Mol}$ Cancer Res 8:569-577.

Kim MR, Jeon ES, Kim YM, Lee JS, and Kim JH (2009) Thromboxane $\mathrm{A}_{2}$ induces differentiation of human mesenchymal stem cells to smooth muscle-like cells. Stem Cells 27:191-199.

Kim S, Bellone S, Maxey KM, Powell WS, Lee GJ, and Rokach J (2005) Synthesis of $15 \mathrm{R}-\mathrm{PGD}_{2}$ : a potential $\mathrm{DP}_{2}$ receptor agonist. Bioorg Med Chem Lett 15:1873-1876. Kinsella BT, O'Mahony DJ, and Fitzgerald GA (1997) The human thromboxane $\mathrm{A}_{2}$ receptor $\alpha$ isoform (TP $\alpha$ ) functionally couples to the $G$ proteins $G_{q}$ and $G_{11}$ in vivo and is activated by the isoprostane 8-epi prostaglandin $\mathrm{F}_{2 \alpha}$.J Pharmacol Exp Ther 281:957-964.

Kitamura N, Hukuda R, Majima T, Horie T, and Sugihara T (2003a) [The 3 effective cases of cough variant asthma with ramotroban.] Arerugi 52:1089-1092.

Kitamura T, Itoh M, Noda T, Tani K, Kobayashi M, Maruyama T, Kobayashi K, Ohuchida S, Sugimura T, and Wakabayashi K (2003b) Combined effects of prostaglandin $\mathrm{E}$ receptor subtype $\mathrm{EP}_{1}$ and subtype $\mathrm{EP}_{4}$ antagonists on intestinal tumorigenesis in adenomatous polyposis coli gene knockout mice. Cancer Sci 94:618-621.

Kitaoka S, Furuyashiki T, Nishi A, Shuto T, Koyasu S, Matsuoka T, Miyasaka M, Greengard $\mathrm{P}$, and Narumiya $\mathrm{S}$ (2007) Prostaglandin $\mathrm{E}_{2}$ acts on $\mathrm{EP}_{1}$ receptor and amplifies both dopamine $\mathrm{D}_{1}$ and $\mathrm{D}_{2}$ receptor signaling in the striatum. $J$ Neurosci 27:12900-12907.

Kobayashi T, Tahara Y, Matsumoto M, Iguchi M, Sano H, Murayama T, Arai H, Oida H, Yurugi-Kobayashi T, Yamashita JK, et al. (2004) Roles of thromboxane $\mathrm{A}_{2}$ and prostacyclin in the development of atherosclerosis in apoE-deficient mice. $J$ Clin Invest 114:784-794.

Kobayashi K, Arakawa T, Higuchi K, and Nakamura H (1991) Gastric cytoprotection by ornoprostil, a $\mathrm{PGE}_{1}$ analogue, in human subjects. J Clin Gastroenterol 13 (Suppl 1):S32-S36.

Kojima F, Kapoor M, Kawai S, Yang L, Aronoff DM, and Crofford LJ (2009) Prostaglandin $\mathrm{E}_{2}$ activates Rap1 via $\mathrm{EP}_{2} / \mathrm{EP}_{4}$ receptors and cAMP-signaling in rheumatoid synovial fibroblasts: involvement of Epac1 and PKA. Prostaglandins Other Lipid Mediat 89:26-33.

Kolodsick JE, Peters-Golden M, Larios J, Toews GB, Thannickal VJ, and Moore BB (2003) Prostaglandin $\mathrm{E}_{2}$ inhibits fibroblast to myofibroblast transition via $\mathrm{E}$. prostanoid receptor 2 signaling and cyclic adenosine monophosphate elevation. Am J Respir Cell Mol Biol 29:537-544.

Kondo K, Machii K, Narita M, Kawamoto A, Yamasaki S, and Hamanaka N (1995) ONO-AP-500-02: A non prostanoid prostaglandin $\mathrm{I}_{2}$ mimetic with inhibitory activity against thromboxane synthase. Adv Prostaglandin Thromboxane Leukot Res 23:401-403.

Konger RL, Billings SD, Prall NC, Katona TM, Dasilva SC, Kennedy CR, Badve S, Perkins SM, and Lacelle PT (2009) The $\mathrm{EP}_{1}$ subtype of the prostaglandin $\mathrm{E}_{2}$ receptor: role in keratinocyte differentiation and expression in non-melanoma skin cancer. Prostaglandins Leukot Essent Fatty Acids 81:279-290.

Konger RL, Billings SD, Thompson AB, Morimiya A, Ladenson JH, Landt Y, Pentland AP, and Badve S (2005a) Immunolocalization of low-affinity prostaglandin E receptors, $\mathrm{EP}_{1}$ in adult human epidermis. J Invest Dermatol 124:965-970.

Konger RL, Brouxhon S, Partillo S, VanBuskirk J, and Pentland AP (2005b) The $\mathrm{EP}_{3}$ receptor stimulates ceramide and diacylglycerol release and inhibits growth of primary keratinocytes. Exp Dermatol 14:914-922.

Konger RL, Scott GA, Landt Y, Ladenson JH, and Pentland AP (2002) Loss of the $\mathrm{EP}_{2}$ prostaglandin $\mathrm{E}_{2}$ receptor in immortalized human keratinocytes results in increased invasiveness and decreased paxillin expression. Am J Pathol 161:2065 2078.

Kostenis E and Ulven T (2006) Emerging roles of DP and CRTH2 in allergic inflammation. Trends Mol Med 12:148-158.

Kotani M, Tanaka I, Ogawa Y, Suganami T, Matsumoto T, Muro S, Yamamoto Y, Sugawara A, Yoshimasa Y, Sagawa N, et al. (2000) Multiple signal transduction pathways through two prostaglandin E receptor EP3 subtype isoforms expressed in human uterus. J Clin Endocrinol Metab 85:4315-4322.

Kotani M, Tanaka I, Ogawa Y, Usui T, Mori K, Ichikawa A, Narumiya S, Yoshimi T, and Nakao K (1995) Molecular cloning and expression of multiple isoforms of human prostaglandin $\mathrm{E}$ receptor $\mathrm{EP}_{3}$ subtype generated by alterative messenger RNA splicing: multiple second messenger systems and tissue-specific distributions. Mol Pharmacol 48:869-879.

Kotani T, Kobata A, Nakamura E, Amagase K, and Takeuchi K (2006) Roles of cyclooxygenase- 2 and prostacyclin/IP receptors in musosal defense against ischemia-reperfusion injury in mouse stomach. J Pharmacol Exp Ther 316:547-555.

Kotelevets L, Foudi N, Louedec L, Couvelard A, Chastre E, and Norel X (2007) A new mRNA splice variant coding for the human EP3-1 receptor isoform. Prostaglandins Leukot Essent Fatty Acids 77:195-201.

Kozaki Y, Kambe F, Hayashi Y, Ohmori S, Seo H, Kumazawa T, and Mizumura K (2007) Molecular cloning of prostaglandin $\mathrm{EP}_{3}$ receptors from canine sensory ganglia and their facilitatory action on bradykinin-induced mobilization of intracellular calcium. J Neurochem 100:1636-1647.

Krause P, Singer E, Darley PI, Klebensberger J, Groettrup M, and Legler DF (2007) Prostaglandin $\mathrm{E}_{2}$ is a key factor for monocyte-derived dendritic cell maturation: enhanced T cell stimulatory capacity despite IDO. J Leukoc Biol 82:1106-1114.

Krauss AH, Woodward DF, Gibson LL, Protzman CE, Williams LS, Burk RM, Gac TS, Roof MB, Abbas F, Marshall K, et al. (1996) Evidence for human thromboxane receptor heterogeneity using a novel series of 9,11-cyclic carbonate derivatives of prostaglandin $\mathrm{F}_{2 \alpha}$. Br J Pharmacol 117:1171-1180.

Kubo S, Takahashi HK, Takei M, Iwagaki H, Yoshino T, Tanaka N, Mori S, and Nishibori $\mathrm{M}$ (2004) E-prostanoid $(\mathrm{EP})_{2} / \mathrm{EP}_{4}$ receptor-dependent maturation of human monocyte-derived dendritic cells and induction of helper T2 polarization. J Pharmacol Exp Ther 309:1213-1220.

Kunikata T, Araki H, Takeeda M, Kato S, and Takeuchi K (2001) Prostaglandin E prevents indomethacin-induced gastric and intestinal damage through different EP receptor subtypes. J Physiol Paris 95:157-163.

Kunikata T, Tanaka A, Miyazawa T, Kato S, and Takeuchi K (2002) 16,16-Dimethyl prostaglandin $\mathrm{E}_{2}$ inhibits indomethacin-induced small intestinal lesions through $\mathrm{EP}_{3}$ and $\mathrm{EP}_{4}$ receptors. Dig Dis Sci 47:894-904.

Kunikata T, Yamane H, Segi E, Matsuoka T, Sugimoto Y, Tanaka S, Tanaka H, Nagai H, Ichikawa A, and Narumiya S (2005) Suppression of allergic inflammation by the prostaglandin E receptor subtype EP3. Nat Immunol 6:524-531.

Kunori S, Matsumura S, Mabuchi T, Tatsumi S, Sugimoto Y, Minami T, and Ito S (2009) Involvement of prostaglandin $\mathrm{F}_{2 \alpha}$ receptor in ATP-induced mechanical allodynia. Neuroscience 163:362-371.

Kurihara Y, Endo H, and Kondo H (2001) Induction of IL-6 via the $\mathrm{EP}_{3}$ subtype of prostaglandin $\mathrm{E}$ receptor in rat adjuvant-arthritic synovial cells. Inflamm Res 50:1-5.

Kuwano K, Hashino A, Asaki T, Hamamoto T, Yamada T, Okubo K, and Kuwabara $\mathrm{K}$ (2007) 2-[4-[(5,6-Diphenylpyrazin-2-yl)(isopropyl)amino]butoxy]- $N$-(methylsulfonyl)acetamide (NS-304), an orally available and long-acting prostacyclin receptor agonist prodrug. J Pharmacol Exp Ther 322:1181-1188.

Kuwano K, Hashino A, Noda K, Kosugi K, and Kuwabara K (2008) A long-acting and highly selective prostacyclin receptor agonist prodrug, $2-\{4-[(5,6$-diphenylpyrazin2 -yl) (isopropyl)amino] butoxy\}- $N$-(methylsulfonyl)acetamide (NS-304), ameliorates rat pulmonary hypertension with unique relaxant responses of its active form, \{4-[(5,6-diphenylpyrazin-2-yl)(isopropyl)amino] butoxy\}acetic acid (MRE269), on rat pulmonary artery. J Pharmacol Exp Ther 326:691-699.

Lai J, Jin H, Yang R, Winer J, Li W, Yen R, King KL, Zeigler F, Ko A, Cheng J, et al. (1996) Prostaglandin $\mathrm{F}_{2 \alpha}$ induces cardiac myocyte hypertrophy in vitro and cardiac growth in vivo. Am J Physiol 271:H2197-H2208. 
Larsen R, Hansen MB, and Bindslev N (2005) Duodenal secretion in humans mediated by the $\mathrm{EP}_{4}$ receptors subtype. Acta Physiol Scand 185:133-140.

Lawler OA, Miggin SM, and Kinsella BT (2001) Protein kinase A-mediated phosphorylation of Serine 357 of the mouse prostacyclin receptor regulates its coupling to $\mathrm{G}_{\mathrm{s}}$-, to $\mathrm{G}_{\mathrm{i}}$, and to $\mathrm{G}_{\mathrm{q}}$-coupled effector signaling. J Biol Chem 276:33596-33607.

Lawrence RA and Jones RL (1992) Investigation of the prostaglandin E (EP-) receptor subtype mediating relaxation of the rabbit jugular vein. $\mathrm{Br} J$ Pharmacol 105:817-824.

Lawrence RA, Jones RL, and Wilson NH (1992) Characterization of receptors involved in the direct and indirect actions of prostaglandins $\mathrm{E}$ and I on the guineapig ileum. Br J Pharmacol 105:271-278.

Lazarus M, Yoshida K, Coppari R, Bass CE, Mochizuki T, Lowell BB, and Saper CB (2007) EP3 prostaglandin receptors in the median preoptic nucleus are critical for fever responses. Nat Neurosci 10:1131-1133.

Lee CM, Genetos DC, You Z, and Yellowley CE (2007a) Hypoxia regulates PGE(2) release and $\mathrm{EP}_{1}$ receptor expression in osteoblastic cells. J Cell Physiol 212:182_ 188

Lee JL, Kim A, Kopelovich L, Bickers DR, and Athar M (2005) Differential expression of $\mathrm{E}$ prostanoid receptors in murine and human non-melanoma skin cancer. $J$ Invest Dermatol 125:818-825.

Lee T, Hedlund P, Newgreen D, and Andersson KE (2007b) Urodynamic effects of a novel $\mathrm{EP}_{1}$ receptor antagonist in normal rats and rats with bladder outlet obstruction. J Urol 177:1562-1567.

Leff $\mathrm{P}$ and Giles H (1992) Classification of platelet and vascular prostaglandin $\mathrm{D}_{2}$ (DP) receptors: estimation of affinities and relative efficacies for a series of novel bicyclic ligands. With an appendix on goodness-of-fit analyses. $\mathrm{Br}$ J Pharmacol 106:996-1003.

Legler DF, Krause P, Scandella E, Singer E, and Groettrup M (2006) Prostaglandin $\mathrm{E}_{2}$ is generally required for human dendritic cell migration and exerts its effect via $\mathrm{EP}_{2}$ and $\mathrm{EP}_{4}$ receptors. J Immunol 176:966-973.

Leone V, di Palma A, Ricchi P, Acquaviva F, Giannouli M, Di Prisco AM, Iuliano F, and Acquaviva AM (2007) $\mathrm{PGE}_{2}$ inhibits apoptosis in human adenocarcinoma Caco-2 cell line through Ras-PI3K association and cAMP-dependent kinase A activation. Am J Physiol Gastrointest Liver Physiol 293:G673-G681.

Leonhardt A, Glaser A, Wegmann M, Hackenberg R, and Nüsing RM (2003) Expression of prostanoid receptors in human lower segment pregnant myometrium. Prostaglandins Leukot Essent Fatty Acids 69:307-313.

Li JH, Chou CL, Li B, Gavrilova O, Eisner C, Schnermann J, Anderson SA, Deng CX, Knepper MA, and Wess $\mathrm{J}$ (2009a) A selective $\mathrm{EP}_{4} \mathrm{PGE}_{2}$ receptor agonist alleviates disease in a new mouse model of X-linked nephrogenic diabetes insipidus. J Clin Invest 119:3115-3126.

Li M, Healy DR, Li Y, Simmons HA, Crawford DT, Ke HZ, Pan LC, Brown TA, and Thompson DD (2005) Osteopenia and impaired fracture healing in aged EP4 receptor knockout mice. Bone 37:46-54.

Li M, Ke HZ, Qi H, Healy DR, Li Y, Crawford DT, Paralkar VM, Owen TA, Cameron $\mathrm{KO}$, Lefker BA, et al. (2003) A novel, non-prostanoid $\mathrm{EP}_{2}$ receptor-selective prostaglandin $\mathrm{E}_{2}$ agonist stimulates local bone formation and enhances fracture healing. J Bone Miner Res 18:2033-2042.

Li M, Thompson DD, and Paralkar VM (2007) Prostaglandin $\mathrm{E}_{2}$ receptors in bone formation. Int Orthop 31:767-772.

Li RC, Cindrova-Davies T, Skepper JN, and Sellers LA (2004) Prostacyclin induces apoptosis of vascular smooth muscle cells by a cAMP-mediated inhibition of extracellular signal-regulated kinase activity and can counteract the mitogenic activity of endothelin-1 or basic fibroblast growth factor. Circ Res 94:759-767.

Li X, Ellman M, Muddasani P, Wang JH, Cs-Szabo G, van Wijnen AJ, and Im HJ (2009b) Prostaglandin $\mathrm{E}_{2}$ and its cognate EP receptors control adult articular cartilage homeostasis and are linked to the pathophysiology of osteoarthritis. Arthritis Rheum 60:513-523.

Li X, Okada Y, Pilbeam CC, Lorenzo JA, Kennedy CR, Breyer RM, and Raisz LG (2000) Knockout of the murine prostaglandin $\mathrm{EP}_{2}$ receptor impairs osteoclastogenesis in vitro. Endocrinology 141:2054-2261.

Li X, Pilbeam CC, Pan L, Breyer RM, and Raisz LG (2002) Effects of prostaglandin E2 on gene expression in primary osteoblastic cells from prostaglandin receptor knockout mice. Bone 30:567-573.

Li X and Tai HH (2009) Activation of thromboxane A(2) receptors induces orphan nuclear receptor Nurr1 expression and stimulates cell proliferation in human lung cancer cells. Carcinogenesis 30:1606-1613.

Liang X, Wang Q, Hand T, Wu L, Breyer RM, Montine TJ, and Andreasson K (2005a) Deletion of the prostaglandin $\mathrm{E}_{2} \mathrm{EP}_{2}$ receptor reduces oxidative damage and amyloid burden in a model of Alzheimer's disease. J Neurosci 25:10180-10187.

Liang X, Wu L, Hand T, and Andreasson K (2005b) Prostaglandin $\mathrm{D}_{2}$ mediates neuronal protection via the $\mathrm{DP}_{1}$ receptor. J Neurochem 92:477-486.

Liang Y, Li C, Guzman VM, Evinger AJ 3rd, Protzman CE, Krauss AH, and Woodward DF (2003) Comparison of prostaglandin F2alpha, bimatoprost (prostamide), and butaprost (EP2 agonist) on Cyr61 and connective tissue growth factor gene expression. J Biol Chem 278:27267-27277.

Liang Y, Woodward DF, Guzman VM, Li C, Scott DF, Wang JW, Wheeler LA, Garst ME, Landsverk K, Sachs G, et al. (2008) Identification and pharmacological characterization of the prostaglandin FP receptor and FP variant complexes. $\mathrm{Br} J$ Pharmacol 154:1079-1093.

Lim H and Dey SK (1997) Prostaglandin $\mathrm{E}_{2}$ receptor subtype $\mathrm{EP}_{2}$ gene expression in the mouse uterus coincides with differentiation of the luminal epithelium for implantation. Endocrinology 138:4599-4606.

Lim H and Dey SK (2002) Minireview: a novel pathway of prostacyclin signalinghanging out with nuclear receptors. Endocrinology 143:3207-3210.

Lin CR, Amaya F, Barrett L, Wang H, Takada J, Samad TA, and Woolf CJ (2006) Prostaglandin $\mathrm{E}_{2}$ receptor $\mathrm{EP}_{4}$ contributes to inflammatory pain hypersensitivity. $J$ Pharmacol Exp Ther 319:1096-1103.

Lin H, Lee JL, Hou HH, Chung CP, Hsu SP, and Juan SH (2008) Molecular mechanisms of the antiproliferative effect of beraprost, a prostacyclin agonist, in murine vascular smooth muscle cells. J Cell Physiol 214:434-441.
Liu D, Wu L, Breyer R, Mattson MP, and Andreasson K (2005) Neuroprotection by the $\mathrm{PGE}_{2} \mathrm{EP}_{2}$ receptor in permanent focal cerebral ischemia. Ann Neurol 57:758 761.

Liu J, Fu Z, Wang Y, Schmitt M, Huang A, Marshall D, Tonn G, Seitz L, Sullivan T Lucy Tang H, et al. (2009) Discovery and optimization of CRTH2 and DP dua antagonists. Bioorg Med Chem Lett 19:6419-6423.

Lo RK, Liu AM, Wise H, and Wong YH (2008) Prostacyclin receptor-induced STAT3 phosphorylation in human erythroleukemia cells is mediated via $\mathrm{G} \alpha_{\mathrm{s}}$ and $\mathrm{G} \alpha_{16}$ hybrid signaling. Cell Signal 20:2095-2106.

Lo RK, Wise H, and Wong YH (2006) Prostacyclin receptor induces STAT1 and STAT3 phosphorylations in human erythroleukemia cells: a mechanism requiring PTX-insensitive G proteins, ERK and JNK. Cell Signal 18:307-317.

Lohse MJ (2010) Dimerization in GPCR mobility and signaling. Curr Opin Pharmacol 10:53-58.

Lovgren AK, Jania LA, Hartney JM, Parsons KK, Audoly LP, Fitzgerald GA, Tilley $\mathrm{SL}$, and Koller BH (2006) COX-2-derived prostacyclin protects against bleomycininduced pulmonary fibrosis. Am J Physiol Lung Cell Mol Physiol 291:L144-L156.

Luft T, Jefford M, Luetjens P, Toy T, Hochrein H, Masterman KA, Maliszewski C, Shortman K, Cebon J, and Maraskovsky E (2002) Functionally distinct dendritic cell (DC) populations induced by physiologic stimuli: prostaglandin $\mathrm{E}_{2}$ regulates the migratory capacity of specific DC subsets. Blood 100:1362-1372.

Lumley P, White BP, and Humphrey PP (1989) GR32191, a highly potent and specific thromboxane $\mathrm{A}_{2}$ receptor blocking drug on platelets and vascular and airways smooth muscle in vitro. Br J Pharmacol 97:783-794.

Lundblad C, Grände PO, and Bentzer P (2008) Increased cortical cell loss and prolonged hemodynamic depression after traumatic brain injury in mice lacking the IP receptor for prostacyclin. J Cereb Blood Flow Metab 28:367-376.

Lydford SJ, McKechnie KC, and Dougall IG (1996) Pharmacological studies on prostanoid receptors in the rabbit isolated saphenous vein: a comparison with the rabbit isolated ear artery. $\mathrm{Br} J$ Pharmacol 117:13-20.

Ma H, Hara A, Xiao CY, Okada Y, Takahata O, Nakaya K, Sugimoto Y, Ichikawa A Narumiya S, and Ushikubi F (2001) Increased bleeding frequency and decreased susceptibility to thromboembolism in mice lacking the prostaglandin $\mathrm{E}$ receptor subtype EP(3). Circulation 104:1176-1180.

Ma X, Kundu N, Rifat S, Walser T, and Fulton AM (2006) Prostaglandin E receptor $\mathrm{EP}_{4}$ antagonism inhibits breast cancer metastasis. Cancer Res 66:2923-2927.

Macias-Perez IM, Zent R, Carmosino M, Breyer MD, Breyer RM, and Pozzi A (2008) Mouse $\mathrm{EP} 3 \alpha, \beta$, and $\gamma$ receptor variants reduce tumor cell proliferation and tumorigenesis in vivo. $J$ Biol Chem 283:12538-12545.

Maher SA, Birrell MA, and Belvisi MG (2009) Prostaglandin $\mathrm{E}_{2}$ mediates cough via the EP3 receptor: implications for future disease therapy. Am J Resp Crit Care Med 180:923-928

Mahmud I, Smith DL, Whyte MA, Nelson JT, Cho D, Tokes LG, Alvarez R, and Willis AL (1984) On the identification and biological properties of prostaglandin $\mathrm{J}_{2}$ Prostaglandins Leukot Med 16:131-146.

Mais DE, DeHoll D, Sightler H, and Halushka PV (1988) Different pharmacologic activities for 13-azapinane thromboxane $\mathrm{A}_{2}$ analogs in platelets and blood vessels. Eur J Pharmacol 148:309-315.

Mais DE, Saussy DL Jr, Chaikhouni A, Kochel PJ, Knapp DR, Hamanaka N, and Halushka PV (1985) Pharmacologic characterization of human and canine thromboxane $\mathrm{A}_{2} /$ prostaglandin $\mathrm{H}_{2}$ receptors in platelets and blood vessels: evidence for different receptors. J Pharmacol Exp Ther 233:418-424.

Makino H, Tanaka I, Mukoyama M, Sugawara A, Mori K, Muro S, Suganami T, Yahata K, Ishibashi R, Ohuchida S, et al. (2002) Prevention of diabetic nephropathy in rats by prostaglandin $\mathrm{E}$ receptor $\mathrm{EP}_{1}$-selective antagonist. $J \mathrm{Am} S o$ Nephrol 13:1757-1765.

Makino S, Zaragoza DB, Mitchell BF, Yonemoto H, and Olson DM (2007) Decidual activation: abundance and localization of prostaglandin F2 alpha receptor (FP mRNA and protein and uterine activation proteins in human decidua at preterm birth and term birth. Placenta 28:557-565.

Malinowska B, Godlewski G, Buczko W, and Schlicker E (1994) EP3 receptormediated inhibition of the neurogenic vasopressor response in pithed rats. Eur $J$ Pharmacol 259:315-319.

Marchese A, Sawzdargo M, Nguyen T, Cheng R, Heng HH, Nowak T, Im DS, Lynch KR, George SR, and O'Dowd BF (1999) Discovery of three novel orphan G proteincoupled receptors. Genomics 56:12-21.

Martin M, Meyer-Kirchrath J, Kaber G, Jacoby C, Flögel U, Schrader J, Rüther U, Schrör K, and Hohlfeld T (2005) Cardiospecific overexpression of the prostaglandin $\mathrm{EP}_{3}$ receptor attenuates ischemia-induced myocardial injury. Circulation 112 $400-406$

Martineau LC, McVeigh LI, Jasmin BJ, and Kennedy CR (2004) p38 MAP kinase mediates mechanically induced COX-2 and $\mathrm{PG} \mathrm{EP}_{4}$ receptor expression in podocytes: implications for the actin cytoskeleton. Am J Physiol Renal Physiol 286: F693-F701.

Maruyama T, Asada M, Shiraishi T, Egashira H, Yoshida H, Maruyama T, Ohuchida S, Nakai H, Kondo K, and Toda M (2002a) Design and synthesis of a selective

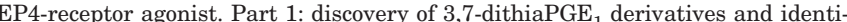
fication of their omega chains. Bioorg Med Chem 10:975-988.

Maruyama T, Asada M, Shiraishi T, Yoshida H, Maruyama T, Ohuchida S, Nakai H, Kondo K, and Toda M (2002b) Design and synthesis of a selective EP4- agonist. Part 3: 16-phenyl-5-thiaPGE 1 and 9- $\beta$-halo derivatives with improved stability. Bioorg Med Chem 10:1743-1759.

Masuda A, Mais DE, Oatis JE Jr, and Halushka PV (1991) Platelet and vascular thromboxane $\mathrm{A}_{2} /$ prostaglandin $\mathrm{H}_{2}$ receptors. Evidence for different subclasses in the rat. Biochem Pharmacol 42:537-544.

Matias I, Chen J, De Petrocellis L, Bisogno T, Ligresti A, Fezza F, Krauss AH, Shi L, Protzman CE, Li C, et al. (2004) Prostaglandin ethanolamides (prostamides): in vitro pharmacology and metabolism). J Pharmacol Exp Ther 309:745-757.

Mathiesen JM, Christopoulos A, Ulven T, Royer JF, Campillo M, Heinemann A, Pardo L, and Kostenis E (2006) On the mechanism of interaction of potent sur- 
mountable and insurmountable antagonists with the prostaglandin $\mathrm{D}_{2}$ receptor CRTH2. Mol Pharmacol 69:1441-1453.

Mathieu MC, Lord-Dufour S, Bernier V, Boie Y, Burch JD, Clark P, Denis D, Han Y, Mortimer JR, and Therien AG (2008) Mutual antagonist relationship between prostaglandin $\mathrm{E}_{2}$ and IFN- $\gamma$ : implications for rheumatoid arthritis. Eur J Immunol 38:1900-1912.

Matlhagela $\mathrm{K}$ and Taub $\mathrm{M}$ (2006) Involvement of $\mathrm{EP}_{1}$ and $\mathrm{EP}_{2}$ receptors in the regulation of the Na,K-ATPase by prostaglandins in MDCK cells. Prostaglandins Other Lipid Mediat 79:101-113.

Matsugi T, Kageyama M, Nishimura K, Giles H, and Shirasawa E (1995) Selective prostaglandin $\mathrm{D}_{2}$ receptor stimulation elicits ocular hypotensive effects in rabbits and cats. Eur J Pharmacol 275:245-250.

Matsumoto S, Ikeda M, Yoshida S, Tanimoto T, Takeda M, and Nasu M (2005) Prostaglandin E2-induced modification of tetrodotoxin-resistant $\mathrm{Na}^{+}$currents involves activation of both EP2 and EP4 receptors in neonatal rat nodose ganglion neurons. Br J Pharmacol 145:503-513.

Matsuo M, Yoshida N, Zaitsu M, Ishii K, and Hamasaki Y (2004) Inhibition of human glioma cell growth by a PHS-2 inhibitor, NS398, and a prostaglandin E receptor subtype $\mathrm{EP}_{1}$-selective antagonist, SC51089. J Neurooncol 66:285-292.

Matsuoka T, Hirata M, Tanaka H, Takahashi Y, Murata T, Kabashima K, Sugimoto Y, Kobayashi T, Ushikubi F, Aze Y, et al. (2000) Prostaglandin $\mathrm{D}_{2}$ as a mediator of allergic asthma. Science 287:2013-2017.

Matsuoka Y, Furuyashiki T, Bito H, Ushikubi F, Tanaka Y, Kobayashi T, Muro S, Satoh N, Kayahara T, Higashi M, et al. (2003) Impaired adrenocorticotropic hormone response to bacteria endotoxin in mice deficient in prostaglandin $\mathrm{E}$ receptor EP1 and EP3 subtypes. Proc Natl Acad Sci USA 100:4132-4137.

Matsuoka Y, Furuyashiki T, Yamada K, Nagai T, Bito H, Tanaka Y, Kitaoka S, Ushikubi F, Nabeshima T, and Narumiya S (2005) Prostaglandin E receptor EP controls impulsive behavior under stress. Proc Natl Acad Sci USA 102:1606616071.

Matthews JS and Jones RL (1993) Potentiation of aggregation and inhibition of adenylate cyclase in human platelets by prostaglandin $\mathrm{E}$ analogues. $\mathrm{Br} J \mathrm{Phar}$ macol 108:363-369.

Maubach KA, Davis RJ, Clark DE, Fenton G, Lockey PM, Clark KL, Oxford AW, Hagan RM, Routledge C, and Coleman RA (2009) BGC20-1531, a novel, potent and selective EP receptor antagonist: putative new treatment for migraine headache. Br J Pharmacol 156:316-327.

McCafferty GP, Misajet BA, Laping NJ, Edwards RM, and Thorneloe KS (2008) Enhanced bladder capacity and reduced prostaglandin E2-mediated bladder hyperactivity in $\mathrm{EP}_{3}$ receptor knockout mice. Am J Physiol Renal Physiol 295:F507F514

McCormick C, Jones RL, Kennedy S, and Wadsworth RM (2010) Activation of prostanoid EP receptors by prostacyclin analogues in the rabbit iliac artery: implications for anti-restenotic potential? Eur J Pharmacol 641:160-167.

McCullough L, Wu L, Haughey N, Liang X, Hand T, Wang Q, Breyer RM, and Andreasson $\mathrm{K}$ (2004) Neuroprotective function of the $\mathrm{PGE}_{2} \mathrm{EP}_{2}$ receptor in cerebral ischemia. J Neurosci 24:257-268.

McGraw DW, Mihlbachler KA, Schwarb MR, Rahman FF, Small KM, Almoosa KF and Liggett SB (2006) Airway smooth muscle prostaglandin-EP $\mathrm{E}_{1}$ receptors directly modulate $\beta_{2}$-adrenergic receptors within a unique heterodimeric complex. J Clin Invest 116:1400-1409.

McIlroy A, Caron G, Blanchard S, Frémaux I, Duluc D, Delneste Y, Chevailler A, and Jeannin P (2006) Histamine and prostaglandin E up-regulate the production of Th2-attracting chemokines (CCL17 and CCL22) and down-regulate IFN-gammainduced CXCL10 production by immature human dendritic cells. Immunology 117:507-516.

McKenniff M, Rodger IW, Norman P, and Gardiner PJ (1988) Characterisation of receptors mediating the contractile effects of prostanoids in guinea-pig and human airways. Eur J Pharmacol 153:149-159.

Meanwell NA, Romine JL, and Seiler SM (1994) Non-prostanoid prostacyclin mimetics. Drugs Future 19:361-385.

Medeiros AI, Serezani CH, Lee SP, and Peters-Golden M (2009) Efferocytosis impairs pulmonary macrophage and lung antibacterial function via $\mathrm{PGE}_{2} / \mathrm{EP}_{2}$ signaling. J Exp Med 206:61-68.

Medina JC and Liu J (2006) PGD antagonists. Annu Rep Med Chem 41:221-235.

Melien O, Thoresen GH, Sandnes D, Ostby E, and Christoffersen T (1998) Activation of p42/p44 mitogen-activated protein kinase by angiotensin II, vasopressin, norepinephrine, and prostaglandin F2alpha in hepatocytes is sustained, and like the effect of epidermal growth factor, mediated through pertussis toxin-sensitive mechanism. J Cell Physiol 175:348-358.

Mendez M and LaPointe MC (2005) $\mathrm{PGE}_{2}$-induced hypertrophy of cardiac myocytes involves $\mathrm{EP}_{4}$ receptor-dependent activation of $\mathrm{p} 42 / 44$ MAPK and EGFR transactivation. Am J Physiol Heart Circ Physiol 288:H2111-H2117.

Merritt JE, Brown AM, Bund S, Cooper DG, Egan JW, Hallam TJ, Heagerty AM, Hickey DM, Kaumann AJ, and Keen M (1991a) Primate vascular responses to octimibate, a non-prostanoid agonist at the prostacyclin receptor. Br J Pharmacol 102:260-266.

Merritt JE, Hallam TJ, Brown AM, Boyfield I, Cooper DG, Hickey DM, JaxaChamiec AA, Kaumann AJ, Keen M, and Kelly E (1991b) Octimibate, a potent non-prostanoid inhibitor of platelet aggregation, acts via the prostacyclin receptor. Br J Pharmacol 102:251-259.

Meyer-Kirchrath J, Martin M, Schooss C, Jacoby C, Flögel U, Marzoll A, Fischer JW, Schrader J, Schrör K, and Hohlfeld T (2009) Overexpression of prostaglandin $\mathrm{EP}_{3}$ receptors activates calcineurin and promotes hypertrophy in the murine heart. Cardiovasc Res 81:310-318.

Michel F, Simonet S, Vayssettes-Courchay C, Bertin F, Sansilvestri-Morel P, Bernhardt F, Paysant J, Silvestre JS, Levy BI, Félétou M, et al. (2008) Altered TP receptor function in isolated, perfused kidneys of nondiabetic and diabetic ApoEdeficient mice. Am J Physiol Renal Physiol 294:F120-F129.

Milne SA and Jabbour HN (2003) Prostaglandin (PG) $\mathrm{F}_{2 \alpha}$ receptor expression and signaling in human endometrium: role of $\mathrm{PGF}_{2 \alpha}$ in epithelial cell proliferation. J Clin Endocrinol Metab 88:1825-1832.

Milton AS and Wendlandt S (1970) A possible role for prostaglandin E1 as a modulator for temperature regulation in the central nervous system of the cat $J$ Physiol (Lond) 207:76P-77P

Minami T, Matsumura S, Mabuchi T, Kobayashi T, Sugimoto Y, Ushikubi F, Ichikawa A, Narumiya S, and Ito S (2003) Functional evidence for interaction between prostaglandin EP3 and kappa-opioid receptor pathways in tactile pain induced by human immunodeficiency virus type-1 (HIV-1) glycoprotein gp120. Neuropharmacology 45:96-105.

Minami T, Nakano H, Kobayashi T, Sugimoto Y, Ushikubi F, Ichikawa A, Narumiya $\mathrm{S}$, and Ito S (2001) Characterization of EP receptor subtypes responsible for prostaglandin $\mathrm{E}_{2}$-induced pain responses by use of $\mathrm{EP}_{1}$ and $\mathrm{EP}_{3}$ receptor knockout mice. Br J Pharmacol 133:438-444.

Minami T, Nishihara I, Sakamoto K, Ito S, Hyodo M, and Hayaishi O (1995) Blockade by ONO-NT-012, a unique prostanoid analogue, of prostaglandin E2 induced allodynia in conscious mice. $\mathrm{Br} J$ Pharmacol 115:73-76.

Minami T, Nishihara I, Uda R, Ito S, Hyodo M, and Hayaishi O (1994) Characterization of EP-receptor subtypes involved in allodynia and hyperalgesia induced by intrathecal administration of prostaglandin $\mathrm{E}_{2}$ to mice. $\mathrm{Br}$ J Pharmacol 112:735740 .

Minami T, Okuda-Ashitaka E, Nishizawa M, Mori H, and Ito S (1997) Inhibition of nociceptin-induced allodynia in conscious mice by prostaglandin $\mathrm{D}_{2} . \mathrm{Br} J$ Phar macol 122:605-610.

Mir F and Le Breton GC (2008) A novel nuclear signaling pathway for thromboxane $\mathrm{A}_{2}$ receptors in oligodendrocytes: evidence for signaling compartmentalization during differentiation. Mol Cell Biol 28:6329-6341.

Mita H, Hasegawa M, Higashi N, and Akiyama K (2002) Characterization of $\mathrm{PGE}_{2}$ receptor subtypes in human eosinophils. $J$ Allergy Clin Immunol 110:457-459.

Mitsumori S (2004) Recent progress in work on $\mathrm{PGD}_{2}$ antagonists for drugs targeting allergic diseases. Curr Pharm Des 10:3533-3538.

Miyamoto M, Ito H, Mukai S, Kobayashi T, Yamamoto H, Kobayashi M, Maruyama T, Akiyama H, and Nakamura T (2003) Simultaneous stimulation of $\mathrm{EP}_{2}$ and $\mathrm{EP}_{4}$ is essential to the effect of prostaglandin $\mathrm{E}_{2}$ in chondrocyte differentiation. Osteoarthritis Cartilage 11:644-652.

Miyata Y, Kanda S, Nomata K, Eguchi J, and Kanetake H (2005) Expression of cyclooxygenase-2 and $\mathrm{EP}_{4}$ receptor in transitional cell carcinoma of the upper urinary tract. $J$ Urol 173:56-60.

Miyatake S, Manabe-Kawaguchi H, Watanabe K, Hori S, Aikawa N, and Fukuda K (2007) Prostaglandin $\mathrm{E}_{2}$ induces hypertrophic changes and suppresses alphaskeletal actin gene expression in rat cardiomyocytes. $J$ Cardiovasc Pharmacol 50:548-554.

Miyaura C, Inada M, Suzawa T, Sugimoto Y, Ushikubi F, Ichikawa A, Narumiya S, and Suda T (2000) Impaired bone resorption to prostaglandin E2 in prostaglandin E receptor EP4-knockout mice. J Biol Chem 275:19819-19823.

Mizoguchi A, Eguchi N, Kimura K, Kiyohara Y, Qu WM, Huang ZL, Mochizuki T, Lazarus M, Kobayashi T, Kaneko T, et al. (2001) Dominant localization of prostaglandin D receptors on arachnoid trabecular cells in mouse basal forebrain and their involvement in the regulation of non-rapid eye movement sleep. Proc Nat Acad Sci USA 98:11674-11679.

Mizutani N, Suda M, Ishimura M, Kurokawa S, Nabe T, and Kohno S (2008) Effects of KP-496, a novel dual antagonist of leukotriene $\mathrm{D}_{4}$ and thromboxane $\mathrm{A}_{2}$ receptors on nasal blockage in guinea pig models of allergic rhinitis. Inflamm Res 57:247251.

Mohri I, Taniike M, Taniguchi H, Kanekiyo T, Aritake K, Inui T, Fukumoto N, Eguchi N, Kushi A, Sasai H, et al. (2006) Prostaglandin $\mathrm{D}_{2}$-mediated microglia/ astrocyte interaction enhances astrogliosis and demyelination in twitcher. $J \mathrm{Neu}$ rosci 26:4383-4393.

Molderings GJ, Colling E, Likungu J, Jakschik J, and Göthert M (1994) Modulation of noradrenaline release from the sympathetic nerves of the human saphenous vein and pulmonary artery by presynaptic $\mathrm{EP}_{3^{-}}$and DP-receptors. $\mathrm{Br}$ J Pharmacol 111:733-738

Momma K, Toyoshima K, Takeuchi D, Imamura S, and Nakanishi T (2005) In vivo reopening of the neonatal ductus arteriosus by a prostanoid $\mathrm{EP}_{4}$-receptor agonist in the rat. Prostaglandins Other Lipid Mediat 78:117-128.

Monneret G, Cossette C, Gravel S, Rokach J, and Powell WS (2003) 15R-Methylprostaglandin $\mathrm{D}_{2}$ is a potent and selective CRTH2/DP 2 receptor agonist in human eosinophils. J Pharmacol Exp Ther 304:349-355.

Monneret G, Gravel S, Diamond M, Rokach J, and Powell WS (2001) Prostaglandin $\mathrm{D}_{2}$ is a potent chemoattractant for human eosinophils that acts via a novel DP receptor. Blood 98:1942-1948.

Monneret G, Li H, Vasilescu J, Rokach J, and Powell WS (2002) 15-Deoxy-delta 12,14-prostaglandins $\mathrm{D}_{2}$ and $\mathrm{J}_{2}$ are potent activators of human eosinophils. $J \mathrm{Im}$ munol 168:3563-3569.

Montine TJ, Milatovic D, Gupta RC, Valyi-Nagy T, Morrow JD, and Breyer RM (2002) Neuronal oxidative damage from activated innate immunity is $\mathrm{EP}_{2}$ receptor-dependent. $J$ Neurochem 83:463-470.

Moore BB, Ballinger MN, White ES, Green ME, Herrygers AB, Wilke CA, Toews GB, and Peters-Golden M (2005) Bleomycin-induced E prostanoid receptor changes alter fibroblast responses to prostaglandin $\mathrm{E}_{2}$. J Immunol 174:5644-5649.

Mori A, Ishii T, Kuroki T, Shigeta N, Sakamoto K, Nakahara T, and Ishii K (2009) The prostanoid $\mathrm{EP}_{2}$ receptor agonist ONO-AE1-259-01 protects against glutamate-induced neurotoxicity in rat retina. Eur J Pharmacol 616:64-67.

Mori A, Saito M, Sakamoto K, Narita M, Nakahara T, and Ishii K (2007) Stimulation of prostanoid IP and $\mathrm{EP}(2)$ receptors dilates retinal arterioles and increases retinal and choroidal blood flow in rats. Eur J Pharmacol 570:135-141.

Morinelli TA, Okwu AK, Mais DE, Halushka PV, John V, Chen CK, and Fried J.K (1989) Difluorothromboxane $\mathrm{A}_{2}$ and stereoisomers: stable derivatives of thromboxane $\mathrm{A}_{2}$ with differential effects on platelets and blood vessels. Proc Natl Acad Sci USA 86:5600-5604.

Moriyama T, Higashi T, Togashi K, Iida T, Segi E, Sugimoto Y, Tominaga T, 
Narumiya S, and Tominaga M (2005) Sensitization of TRPV1 by EP1 and IP reveals peripheral nociceptive mechanism of prostaglandins. Mol Pain 1:3.

Morsy MA, Isohama Y, and Miyata T (2001) Prostaglandin E(2) increases surfactant secretion via the EP(1) receptor in rat alveolar type II cells. Eur J Pharmacol 426:21-24.

Moussa O, Ashton AW, Fraig M, Garrett-Mayer E, Ghoneim MA, Halushka PV, and Watson DK (2008) Novel role of thromboxane receptors $\beta$ isoform in bladder cancer pathogenesis. Cancer Res 68:4097-4104.

Mubarak KK (2010) A review of prostaglandin analogs in the management of patients with pulmonary arterial hypertension. Respir Med 104:9-21.

Muja N, Nelson JK, and DeVries GH (2007) Schwann cells express IP prostanoid receptors coupled to an elevation in intracellular cyclic AMP. J Neurosci Res 85:1159-1169

Muller M, Sales KJ, Katz AA, and Jabbour HN (2006) Seminal plasma promotes the expression of tumorigenic and angiogenic genes in cervical adenocarcinoma cells via the E-series prostanoid 4 receptor. Endocrinology 147:3356-3365.

Murata T, Lin MI, Aritake K, Matsumoto S, Narumiya S, Ozaki H, Urade Y, Hori M, and Sessa WC (2008) Role of prostaglandin $\mathrm{D}_{2}$ receptor DP as a suppressor of tumor hyperpermeability and angiogenesis in vivo. Proc Natl Acad Sci USA 105:20009-20014

Murata T, Ushikubi F, Matsuoka T, Hirata M, Yamasaki A, Sugimoto Y, Ichikawa A, Aze Y, Tanaka T, Yoshida N, Ueno A, Oh-ishi S, and Narumiya S (1997) Altered pain perception and inflammation response in mice lacking prostacyclin receptor. Nature 388:678-682.

Muratani T, Nishizawa M, Matsumura S, Mabuchi T, Abe K, Shimamoto K, Minami $\mathrm{T}$, and Ito $\mathrm{S}$ (2003) Functional characterization of prostaglandin $\mathrm{F}_{2 \alpha}$ receptor in the spinal cord for tactile pain (allodynia). J Neurochem 86:374-382.

Murn J, Alibert O, Wu N, Tendil S, and Gidrol X (2008) Prostaglandin $\mathrm{E}_{2}$ regulates B cell proliferation through a candidate tumor suppressor, Ptger 4. J Exp Med 205:3091-3103.

Mutoh M, Watanabe K, Kitamura T, Shoji Y, Takahashi M, Kawamori T, Tani K, Kobayashi M, Maruyama T, Kobayashi K, et al. (2002) Involvement of prostaglandin $\mathrm{E}$ receptor subtype $\mathrm{EP}_{4}$ in colon carcinogenesis. Cancer Res 62:28-32.

Nagamachi M, Sakata D, Kabashima K, Furuyashiki T, Murata T, Segi-Nishida E, Soontrapa K, Matsuoka T, Miyachi Y, and Narumiya S (2007) Facilitation of Th1-mediated immune response by prostaglandin $\mathrm{E}_{\text {receptor }} \mathrm{EP}_{1}$. J Exp Med 204:2865-2874.

Nagamatsu T, Imai H, Yokoi M, Nishiyama T, Hirasawa Y, Nagao T, and Suzuki Y (2006) Protective effect of prostaglandin $\mathrm{EP}_{4}$-receptor agonist on anti-glomerular basement membrane antibody-associated nephritis. J Pharmacol Sci 102:182 188 .

Naganawa A, Matsui T, Ima M, Yoshida K, Tsuruta H, Yamamoto S, Yamamoto H, Okada H, Maruyama T, Nakai H, et al. (2006) Optimization of sulfonamide derivatives as highly selective $\mathrm{EP}_{1}$ receptor antagonists. Bioorg Med Chem 14: $7774-7789$

Nagano T, Kimura SH, and Takemura M (2008) Prostaglandin $\mathrm{E}_{2}$ reduces extracellular ATP-induced migration in cultured rat microglia. Brain Res 1221:1-5.

Nagata K, Tanaka K, Ogawa K, Kemmotsu K, Imai T, Yoshie O, Abe H, Tada K, Nakamura M, Sugamura K, et al. (1999) Selective expression of a novel surface molecule by human Th2 cells in vivo. J Immunol 162:1278-1286.

Naka M, Mais DE, Morinelli TA, Hamanaka N, Oatis JE Jr, and Halushka PV (1992) 7-[(1R,2S,3S,5R)-6,6-dimethyl-3-(4-iodobenzenesulfonylamino)bicyclo[3.1.1]hept$2-4 y l]-5(Z)$-heptenoic acid: a novel high-affinity radiolabeled antagonist for platelet thromboxane $\mathrm{A}_{2}$ /prostaglandin $\mathrm{H}_{2}$ receptors. J Pharmacol Exp Ther 262:632637.

Nakae K, Hayashi F, Hayashi M, Yamamoto N, Iino T, Yoshikawa S, and Gupta J (2005) Functional role of prostacyclin receptor in rat dorsal root ganglion neurons. Neurosci Lett 388:132-137.

Nakagawa O, Tanaka I, Usui T, Harada M, Sasaki Y, Itoh H, Yoshimasa T, Namba T, Narumiya S, and Nakao K (1994) Molecular cloning of human prostacyclin receptor cDNA and its gene expression in the cardiovascular system. Circulation 90:1643-1647.

Nakagawa T, Minami M, Katsumata S, Ienaga Y, and Satoh M (1995) Suppression of naloxone-precipitated withdrawal jumps in morphine-dependent mice by stimulation of prostaglandin $\mathrm{EP}_{3}$ receptor. Br J Pharmacol 116:2661-2666.

Nakahata N (2008) Thromboxane $\mathrm{A}_{2}$ : physiology/pathology, cellular signal transduction and pharmacology. Pharmacol Ther 118:18-35.

Nakajima M, Goh Y, Azuma I, and Hayaishi O (1991) Effects of prostaglandin $D_{2}$ and its analogue, BW245C, on intraocular pressure in humans. Graefes Arch Clin Exp Ophthalmol 229:411-413.

Nakamura Y, Nakamura K, Matsumura K, Kobayashi S, Kaneko T, and Morrison SF (2005) Direct pyrogenic input from prostaglandin $\mathrm{EP}_{3}$ receptor-expressing preoptic neurons to the dorsomedial hypothalamus. Eur J Neurosci 22:3137-3146.

Nakamura Y, Nakamura K, and Morrison SF (2009) Different populations of prostaglandin $\mathrm{EP}_{3}$ receptor-expressing preoptic neurons project to two fever-mediating sympathoexcitatory brain regions. Neuroscience 161:614-620.

Nakao A, Watanabe T, Taniguchi S, Nakamura M, Honda Z, Shimizu T, and Kurokawa $\mathrm{K}$ (1993) Characterization of prostaglandin $\mathrm{F}_{2 \alpha}$ receptor of mouse 3T3 fibroblasts and its functional expression in Xenopus laevis oocytes. J Cell Physiol 155:257-264

Nakayama Y, Omote K, and Namiki A (2002) Role a prostaglandin receptor $\mathrm{EP}_{1}$ in the spinal dorsal horn in carrageenan-induced inflammatory pain. Anesthesiology 97:1254-1262.

Nakayama Y, Omote K, Kawamata T, and Namiki A (2004) Role of prostaglandin receptor subtype $\mathrm{EP}_{1}$ in prostaglandin $\mathrm{E}_{2}$-induced nociceptive transmission in the rat spinal dorsal horn. Brain Res 1010:62-68.

Namba T, Oida H, Sugimoto Y, Kakizuka A, Negishi M, Ichikawa A, and Narumiya $\mathrm{S}$ (1994) cDNA cloning of a mouse prostacyclin receptor. Multiple signaling pathways and expression in thymic medulla. J Biol Chem 269:9986-9992.

Namba T, Sugimoto Y, Negishi M, Irie A, Ushikubi F, Kakizuka A, Ito S, Ichikawa
A, and Narumiya S (1993) Alternative splicing of C-terminal tail of prostaglandin $\mathrm{E}$ receptor subtype $\mathrm{EP}_{3}$ determines G-protein specificity. Nature 365:166-170.

Napolitani G, Acosta-Rodriguez EV, Lanzavecchia A, and Sallusto F (2009) Prostaglandin $\mathrm{E}_{2}$ enhances Th17 responses via modulation of IL-17 and $\mathrm{IFN}_{\gamma}$-production by memory CD4 ${ }^{+}$T cells. Eur J Immunol 39:1301-1312.

Narisada M, Ohtani M, Watanabe F, Uchida K, Arita H, Doteuchi M, Hanasaki K, Kakushi H, Otani K, and Hara S (1988) Synthesis and in vitro activity of various derivatives of a novel thromboxane receptor antagonist, (+/-)-(5Z)-7-[3-endo[(phenylsulfonyl)amino]bicyclo[2.2.1] hept-2-exo-yl]heptenoic acid. J Med Chem 31:1847-1854

Narko K, Ritvos O, and Ristimäki A (1997) Induction of cyclooxygenase-2 and prostaglandin $\mathrm{F}_{2 \alpha}$ receptor expression by interleukin- $1 \beta$ in cultured human granulosa-luteal cells. Endocrinology 138:3638-3644.

Narumiya S (2003) Prostanoids in immunity: roles revealed by mice deficient in their receptors. Life Sci 74:391-395.

Narumiya S (2009) Prostanoids and inflammation: a new concept arising from receptor knockout mice. J Mol Med 87:1015-1022.

Narumiya S and Toda N (1985) Different responsiveness of prostaglandin $D_{2}$ sensitive systems to prostaglandin $\mathrm{D}_{2}$ and its analogues. $\mathrm{Br}$ J Pharmacol 85:367375 .

Narumiya S, Sugimoto Y, and Ushikubi F (1999) Prostanoid receptors: structures, properties, and functions. Physiol Rev 79:1193-1226.

Nasrallah R and Hébert RL (2005) Prostacyclin signaling in the kidney: implications for health and disease. Am J Physiol Renal Physiol 289:F235-F246.

Nasrallah R, Zimpelmann J, Singh S, and Hébert RL (2001) Molecular and biochemical characterization of prostacyclin receptors in rat kidney. Am J Physiol Renal Physiol 280:F266-F277.

Nataraj C, Thomas DW, Tilley SL, Nguyen MT, Mannon R, Koller BH, and Coffman TM (2001) Receptor for prostaglandin $\mathrm{E}_{2}$ that regulate cellular immune response in the mouse. $J$ Clin Invest 108:1229-1235.

Needleman P, Moncada S, Bunting S, Vane JR, Hamberg M, and Samuelsson B (1976) Identification of an enzyme in platelet microsomes which generates thromboxane $\mathrm{A}_{2}$ from prostaglandin endoperoxides. Nature 261:558-560.

Negishi M, Harazono A, Sugimoto Y, Hazato A, Kurozumi S, and Ichikawa A (1995 Selective coupling of prostaglandin E receptor EP3D to multiple G proteins depending on interactions of the carboxylic acid of agonist and arginine residue of seventh transmembrane domain. Biochem Biophys Res Commun 212:279-285.

Negishi M, Hasegawa H, and Ichikawa A (1996) Prostaglandin E receptor EP3 isoform, with mostly full constitutive Gi activity and agonist-dependent Gs activity. FEBS Lett 386:165-168.

Ney P and Schrör K (1991) PGD $_{2}$ and its mimetic ZK 110.841 are potent inhibitors of receptor-mediated activation of human neutrophils. Eicosanoids 4:21-28.

Nguyen M, Camenisch T, Snouwaert JN, Hicks E, Coffman TM, Anderson PA Malouf NN, and Koller $\mathrm{BH}$ (1997) The prostaglandin receptor $\mathrm{EP}_{4}$ triggers remodelling of the cardiovascular system at birth. Nature 390:78-81.

Nguyen M, Pace AJ, and Koller BH (2005) Age-induced reprogramming of mast cell degranulation. J Immunol 175:5701-5707.

Nguyen M, Solle M, Audoly LP, Tilley SL, Stock JL, McNeish JD, Coffman TM, Dombrowicz D, and Koller BH (2002) Receptors and signaling mechanisms required for prostaglandin $\mathrm{E}_{2}$-mediated regulation of mast cell degranulation and IL-6 production. I Immunol 169:4586-4593.

Nials AT, Vardey CJ, Denyer LH, Thomas M, Sparrow SJ, Shepherd GD, and Coleman RA (1993) AH 13205, a selective prostanoid $\mathrm{EP}_{2}$-receptor agonist. Car diovasc Drug Rev 11:165-179.

Nicola C, Timoshenko AV, Dixon SJ, Lala PK, and Chakraborty C (2005) EP receptor-mediated migration of the first trimester extravillous trophoblast: the role of intracellular calcium and calpain. J Clin Endocrinol Metab 90:4736-4746

Nie D, Guo Y, Yang D, Tang Y, Chen Y, Wang MT, Zacharek A, Qiao Y, Che M, and Honn KV (2008) Thromboxane $A_{2}$ receptors in prostate carcinoma: expression and its role in regulating cell motility via small GTPase Rho. Cancer Res 68:115-121.

Nie D, Lamberti M, Zacharek A, Li L, Szekeres K, Tang K, Chen Y, and Honn KV (2000) Thromboxane $A_{2}$ regulation of endothelial cell migration, angiogenesis, and tumor metastasis. Biochem Biophys Res Commun 267:245-251.

Nilsson SF, Drecoll E, Lütjen-Drecoll E, Toris CB, Krauss AH, Kharlamb A, Nieves $\mathrm{A}$, Guerra $\mathrm{T}$, and Woodward DF (2006) The prostanoid $\mathrm{EP}_{2}$ receptor agonist butaprost increases uveoscleral outflow in the cynomolgus monkey. Invest Ophthalmol Vis Sci 47:4042-4049.

Nitta M, Hirata I, Toshina K, Murano M, Maemura K, Hamamoto N, Sasaki S, Yamauchi H, and Katsu K (2002) Expression of the EP4 prostaglandin E2 recepto subtype with rat dextran sodium sulphate colitis: colitis suppression by a selective agonist, ONO-AEI-329. Scand J Immunol 56:66-75

Nobles M, Benians A, and Tinker A (2005) Heterotrimeric G proteins precouple with G protein-coupled receptors in living cells. Proc Natl Acad Sci USA 102:18706 18711.

Noguchi K, Shitashige M, Endo H, Kondo H, and Ishikawa I (2002) Binary regulation of interleukin (IL)- 6 production by $\mathrm{EP}_{1}$ and $\mathrm{EP}_{2 / 4}$ subtypes of $\mathrm{PGE}_{2}$ receptors in IL-1beta-stimulated human gingival fibroblasts. J Periodontal Res 37:29-36.

Nomiya R, Okano M, Fujiwara T, Maeda M, Kimura Y, Kino K, Yokoyama M, Hira H, Nagata K, Hara T, et al. (2008) CRTH2 plays an essential role in the pathophysiology and Cry ${ }_{j} 1$-induced pollinosis in mice. J Immunol 180:5680-5688.

Norel X, de Montpreville V, and Brink C (2004) Vasoconstriction induced by activation of $\mathrm{EP}_{1}$ and $\mathrm{EP}_{3}$ receptors in human lung: effects of ONO-AE-248, ONO-DI004, ONO-8711 or ONO-8713. Prostaglandins Other Lipid Mediat 74:101-112.

Norel X, Walch L, Labat C, Gascard JP, Dulmet E, and Brink C (1999) Prostanoid receptors involved in the relaxation of human bronchial preparations. $\mathrm{Br} J \mathrm{Phar}$ macol 126:867-872.

O'Connell M, Zeller W, Burgeson J, Mishra RK, Ramirez J, Kiselyov AS, Andrésson T, Gurney ME, and Singh J (2009) Peri-substituted hexahydro-indolones as novel, potent and selective human $\mathrm{EP}_{3}$ receptor antagonists. Bioorg Med Chem Let 19:778-782.

Ochsner SA, Russell DL, Day AJ, Breyer RM, and Richards JS (2003) Decreased 
expression of tumor necrosis factor- $\alpha$-stimulated gene 6 in cumulus cells of the cyclooxygenase-2 and $\mathrm{EP}_{2}$ null mice. Endocrinology 144:1008-1019.

Ocklind A (1998) Effect of latanoprost on the extracellular matrix of the ciliary muscle: a study on cultured cells and tissue sections. Exp Eye Res 67:179-191.

Oelz O, Oelz R, Knapp HR, Sweetman BJ, and Oates JA (1977) Biosynthesis of prostaglandin $\mathrm{D}_{2}$. 1 . Formation of prostaglandin $\mathrm{D}_{2}$ by human platelets. Prostaglandins 13:225-234.

Offermanns S (2006) Activation of platelet function through G-protein-coupled receptors. Circ Res 99:1293-1304.

Offermanns S and Simon MI (1995) $\mathrm{G}_{\alpha 15}$ and $\mathrm{G}_{\alpha 16}$ couple a wide variety of receptors to phospholipase C. J Biol Chem 270:15175-15180.

Oga T, Matsuoka T, Yao C, Nonomura K, Kitaoka S, Sakata D, Kita Y, Tanizawa K, Taguchi Y, Chin K, et al. (2009) Prostaglandin $\mathrm{F}_{2 \alpha}$ receptor signaling facilitates bleomycin-induced pulmonary fibrosis independently of transforming growth factor- $\beta$. Nature Med 15:1426-1430.

Ogletree ML, Harris DN, Schumacher WA, Webb ML, and Misra RN (1993) Pharmacological profile of BMS 180,291: a potent, long-acting, orally active thromboxane $\mathrm{A}_{2}$ /prostaglandin endoperoxide receptor antagonist. J Pharmacol Exp Ther 264:570-578.

Oh DJ, Martin JL, Williams AJ, Russell P, Birk DE, and Rhee DJ (2006) Effect of latanoprost on the expression of matrix metalloproteinases and their tissue inhibitors in human trabecular meshwork cells. Invest Ophthalmol Vis Sci 47:38873895

Oh JM, Kim SH, Lee YI, Seo M, Kim SY, Song YS, Kim WH, and Juhnn YS (2009) Human papillomavirus E5 protein induces expression of the $\mathrm{EP}_{4}$ subtype of prostaglandin E2 receptor in cyclic AMP response element-dependent pathways in cervical cancer cells. Carcinogenesis 30:141-149.

Ohnishi A, Shimamoto C, Katsu K, Ito S, Imai Y, and Nakahari T (2001) EP1 and EP4 receptors mediate exocytosis evoked by prostaglandin $\mathrm{E}(2)$ in guinea-pig antral mucous cells. Exp Physiol 86:451-460.

Oiwa M, Satoh T, Watanabe M, Niwa H, Hirai H, Nakamura M, and Yokozeki H (2008) CRTH2-dependent, STAT6-independent induction of cedar pollen dermatitis. Clin Exp Allergy 38:1357-1366.

Oka T, Aou S, and Hori T (1994) Intracerebroventricular injection of prostaglandin $\mathrm{E}_{2}$ induces thermal hyperalgesia in rats: the possible involvement of $\mathrm{EP}_{3}$ receptors. Brain Res 663:287-292.

Oka T and Hori T (1994) $\mathrm{EP}_{1}$-receptor mediation of prostaglandin $\mathrm{E}_{2}$-induced hyperthermia in rats. Am J Physiol 267:R289-R294.

Oka T, Hori T, Hosoi M, Oka K, Abe M, and Kubo C (1997) Biphasic modulation in the trigeminal nociceptive neuronal responses by the intracerebroventricular prostaglandin $\mathrm{E}_{2}$ may be mediated through different $\mathrm{EP}$ receptors subtypes in rats. Brain Res 771:278-284.

Oka T, Oka K, Kobayashi T, Sugimoto Y, Ichikawa A, Ushikubi F, Narumiya S, and Saper CB (2003a) Characteristics of thermoregulatory and febrile responses in mice deficient in prostaglandin $\mathrm{EP}_{1}$ and $\mathrm{EP}_{3}$ receptors. J Physiol 551:945-954.

Oka T, Oka K, and Saper CB (2003b) Contrasting effects of E type prostaglandin (EP) receptor agonists on core body temperature in rats. Brain Res 968:256-262.

Okada Y, Hara A, Ma H, Xiao CY, Takahata O, Kohgo Y, Narumiya S, and Ushikubi F (2000) Characterization of prostanoid receptors mediating contraction of the gastric fundus and ileum: studies using mice deficient in prostanoid receptors. $\mathrm{Br} J$ Pharmacol 131:745-755.

Okano M, Sugata Y, Fujiwara T, Matsumoto R, Nishibori M, Shimizu K, Maeda M, Kimura Y, Kariya S, Hattori H, et al. (2006) E prostanoid $2\left(\mathrm{EP}_{2}\right) / \mathrm{EP}_{4}$-mediated suppression of antigen specific human $\mathrm{T}$-cell responses by prostaglandin $\mathrm{E}_{2}$. Immunology 118:343-352.

Okumura T, Murata Y, Taniguchi K, Murase A, and Nii A (2008) Effects of the selective $\mathrm{EP}_{4}$ antagonist, CJ-023,423 on chronic inflammation and bone destruction in rat adjuvant-induced arthritis. J Pharm Pharmacol 60:723-730.

Okuyama T, Ishihara S, Sato H, Rumi MA, Kawashima K, Miyaoka Y, Suetsugu H, Kazumori H, Cava CF, Kadowaki Y, et al. (2002) Activation of prostaglandin $\mathrm{E}_{2}$-receptor $\mathrm{EP}_{2}$ and $\mathrm{EP}_{4}$ pathways induces growth inhibition in human gastric carcinoma cell lines. J Lab Clin Med 140:92-102.

Oliva P, Berrino L, de Novellis V, Palazzo E, Marabese I, Siniscalco D, Scafuro M, Mariani L, Rossi F, and Maione S (2006) Role of periaqueductal grey prostaglandin receptors in formalin-induced hyperalgesia. Eur $J$ Pharmacol 530:40-47.

Olschewski H, Rose F, Schermuly R, Ghofrani HA, Enke B, Olschewski A, and Seeger W (2004) Prostaglandin and its analogues in the treatment of pulmonary hypertension. Pharmacol Ther 102:139-153.

Olson DM (2005) The promise of prostaglandins: have they fulfilled their potential as therapeutic targets for the delay of preterm birth? J Soc Gynecol Investig 12:466478.

Omote K, Kawamata T, Nakayama Y, Kawamata M, Hazama K, and Namiki A (2001) The effects of peripheral administration of a novel selective antagonist for prostaglandin E receptor subtype $\mathrm{EP}(1)$, ONO-8711, in a rat model of postoperative pain. Anesth Analg 92:233-238.

Omote K, Yamamoto H, Kawamata T, Nakayama Y, and Namiki A (2002) The effects of intrathecal administration of an antagonist for prostaglandin $\mathrm{E}$ receptor subtype $\mathrm{EP}(1)$ on the mechanical and thermal hyperalgesia is a rat model of postoperative pain. Anesth Analg 95:1708-1712.

Ono K, Kaneko H, Choudhary S, Pilbeam CC, Lorenzo JA, Akatsu T, Kugai N, and Raisz LG (2005 Biphastic effect of prostaglandin E2 on osteoclast formation in spleen cell cultures: role of the EP2 receptor. J Bone Miner Res 20:23-29.

Osborne NN, Li GY, Ji D, Andrade da Costa BL, Fawcett RJ, Kang KD, and Rittenhouse KD (2009) Expression of prostaglandin $\mathrm{PGE}_{2}$ receptors under conditions of aging and stress and the protective effect of the $\mathrm{EP}_{2}$ agonist butaprost on retinal ischemia. Invest Ophthalmol Vis Sci 50:3238-3248.

Ostrom RS, Gregorian C, Drenan RM, Xiang Y, Regan JW, and Insel PA (2001) Receptor number and caveolar co-localization determine receptor coupling efficiency to adenylyl cyclase. J Biol Chem 276:42063-42069.

Ota T, Aihara M, Narumiya S, and Araie M (2005) The effects of prostaglandin analogues on IOP in prostanoid FP-receptor-deficient mice. Invest Ophthalmol Vis Sci 46:4159-4163.

Pan MR, Hou MF, Chang HC, and Hung WC (2008) Cyclooxygenase-2 up-regulates CCR7 via $\mathrm{EP}_{2} / \mathrm{EP}_{4}$ receptor signaling pathways to enhance lymphatic invasion of breast cancer cells. J Biol Chem 283:11155-11163.

Paolini JF, Mitchel YB, Reyes R, Kher U, Lai E, Watson DJ, Norquist JM, Meehan AG, Bays HE, Davidson M, et al. (2009) Effects of laropiprant on nicotinic acidinduced flushing in patients with dyslipidemia. Am J Cardiol 101:625-630.

Paralkar VM, Borovecki F, Ke HZ, Cameron KO, Lefker B, Grasser WA, Owen TA Li M, DaSilva-Jardine P, Zhou M, et al. (2003) An $\mathrm{EP}_{2}$ receptor-selective prostaglandin $\mathrm{E}_{2}$ agonist induces bone healing. Proc Natl Acad Sci USA 100:6736-6740.

Parekh A, Sandulache VC, Lieb AS, Dohar JE, and Hebda PA (2007) Differential regulation of free-floating collagen gel contraction by human fetal and adult dermal fibroblasts in response to prostaglandin $\mathrm{E}_{2}$ mediated by an $\mathrm{EP}_{2} / \mathrm{cAMP}$ dependent mechanism. Wound Repair Regen 15:390-398.

Parent JL, Labrecque P, Orsini MJ, and Benovic JL (1999) Internalization of the $\mathrm{TXA}_{2}$ receptor $\alpha$ and $\beta$ isoforms. Role of the differentially spliced $\mathrm{COOH}$ terminus in agonist-promoted receptor internalization. J Biol Chem 274:8941-8948.

Park DW, Baek K, Lee JG, Park YK, Kim JH, Kim JR, and Baek SH (2007) Activation of toll-like receptor 4 modulates vascular endothelial growth factor synthesis through prostacyclin-IP signaling. Biochem Biophys Res Commun 362 $1090-1095$.

Pekcec A, Unkrüer B, Schlichtiger J, Soerensen J, Hartz AM, Bauer B, van Vliet EA, Gorter JA, and Potschka $\mathrm{H}$ (2009) Targeting prostaglandin $\mathrm{E}_{2} \mathrm{EP}_{1}$ receptor prevents seizure-associated P-glycoprotein up-regulation. J Pharmacol Exp Ther 330:939-947.

Peri K, Polyak F, Lubell W, Thouin E, and Chemtob S (2009), inventors; Universite de Montreal, Theratechnologies, Inc., assignees. Peptides and peptidomimetics useful for inhibiting the activity of prostaglandin F $2 \alpha$ receptor. U.S. patent 7,521,530 B2. 2009 Apr 21

Perkins WE, Burton EG, Tsai BS, Collins PW, Casler JJ, Gasiecki AF, Bauer RF, Jones PH, and Gaginella TS (1991) SC-46275: a potent, long-acting gastric antisecretory prostaglandin with low oral bioavailability in the dog. J Pharmacol Exp Ther 259:1004-1007.

Pettipher R (2008) The roles of the prostaglandin $\mathrm{D}_{2}$ receptors $\mathrm{DP}_{1}$ and $\mathrm{CRTH}_{2}$ in promoting allergic responses. Br J Pharmacol 153:S191-S199.

Philip G, van Adelsberg J, Loeys T, Liu N, Wong P, Lai E, Dass SB, and Reiss TF (2009) Clinical studies on the $\mathrm{DP}_{1}$ antagonist laropiprant in asthma and allergic rhinitis. J Allergy Clin Immunol 124:942-8.e1-9.

Pierce KL, Fujino H, Srinivasan D, and Regan JW (1999) Activation of FP prostanoid receptor isoforms leads to Rho-mediated changes in cell morphology and in the cell cytoskeleton. J Biol Chem 274:35944-35949.

Pierce KL, Gil DW, Woodward DF, and Regan JW (1995) Cloning of human prostanoid receptors. Trends Pharmacol Sci 16:253-256.

Piper PJ and Samhoun MN (1981) The mechanism of action of leukotrienes $\mathrm{C}_{4}$ and $\mathrm{D}_{4}$ in guinea-pig isolated perfused lung and parenchymal strips of guinea pig, rabbit and rat. Prostaglandins 21:793-803.

Pollock WK, Armstrong RA, Brydon LJ, Jones RL, and MacIntyre DE (1984) Thromboxane-induced phosphatidate formation in human platelets. Relationship to receptor occupancy and to changes in cytosolic free calcium. Biochem J 219:833-842.

Pönicke K, Giessler C, Grapow M, Heinroth-Hoffmann I, Becker K, Osten B, and Brodde OE (2000) FP-receptor mediated trophic effects of prostanoids in rat ventricular cardiomyocytes. Br J Pharmacol 129:1723-1731.

Pooler AM, Arjona AA, Lee RK, and Wurtman RJ (2004) Prostaglandin $\mathrm{E}_{2}$ regulates amyloid precursor protein expression via the $\mathrm{EP}_{2}$ receptor in cultured rat microglia. Neurosci Lett 362:127-130.

Popp L, Häussler A, Olliges A, Nüsing R, Narumiya S, Geisslinger G, and Tegeder (2009) Comparison of nociceptive behavior in prostaglandin E, F, D, prostacyclin and thromboxane receptor knockout mice. Eur J Pain 13:691-703.

Pozzi A, Yan X, Macias-Perez I, Wei S, Hata AN, Breyer RM, Morrow JD, and Capdevila JH (2004) Colon carcinoma cell growth is associated with prostaglandin $\mathrm{E}_{2} / \mathrm{EP}_{4}$ receptor-evoked ERK activation. J Biol Chem 279:29797-29804.

Prasanna G, Fortner J, Xiang C, Zhang E, Carreiro S, Anderson S, Sartnurak S, Wu G, Gukasyan H, Niesman M, et al. (2009) Ocular pharmacokinetics and hypotensive activity of $\mathrm{PF}-04475270$, an $\mathrm{EP}_{4}$ prostaglandin agonist in preclinical models. Exp Eye Res 89:608-617.

Prinster SC, Hague C, and Hall RA (2005) Heterodimerization of G protein-coupled receptors: specificity and functional significance. Pharmacol Rev 57:289-298.

Pulichino AM, Rowland S, Wu T, Clark P, Xu D, Mathieu MC, Riendeau D, and Audoly LP (2006) Prostacyclin antagonism reduces pain and inflammation in rodent models of hyperalgesia and chronic arthritis. J Pharmacol Exp Ther 319: 1043-1050.

Qian JY, Harding P, Liu Y, Shesely E, Yang XP, and LaPointe MC (2008) Reduced cardiac remodeling and function in cardiac-specific $\mathrm{EP}_{4}$ receptor knockout mice with myocardial infarction. Hypertension 51:560-566.

Qian JY, Leung A, Harding P, and LaPointe MC (2006) PGE $_{2}$ stimulates human brain natriuretic peptide expression via $\mathrm{EP}_{4}$ and p42/44 MAPK. Am J Physiol Heart Circ Physiol 290:H1740-H1746.

Qian YM, Jones RL, Chan KM, Stock AI, and Ho JK (1994) Potent contractile actions of prostanoid $\mathrm{EP}_{3}$-receptor agonists on human isolated pulmonary artery. $\mathrm{Br} J$ Pharmacol 113:369-374.

Qu WM, Huang ZL, Xu XH, Aritake K, Eguchi N, Nambu F, Narumiya S, Urade Y, and Hayaishi O (2006) Lipocalin-type prostaglandin D synthase produces prostaglandin D2 involved in regulation of physiological sleep. Proc Natl Acad Sci USA 103:17949-17954.

Qualtrough D, Kaidi A, Chell S, Jabbour HN, Williams AC, and Paraskeva C (2007) Prostaglandin $\mathrm{F}_{2 \alpha}$ stimulates motility and invasion in colorectal tumor cells. Int $J$ Cancer 121:734-740.

Rachmilewitz D, Chapman JW, and Nicholson PA (1986) A multi-center international controlled comparison of two dosage regimens of misoprostol with cimeti- 
dine in treatment of gastric ulcer in outpatients. Dig Dis Sci 31 (2 Suppl):75S$80 \mathrm{~S}$

Racké K, Bähring J, Langer C, Bräutigam M, and Wessler I (1992) Prostanoids inhibit release of endogenous norepinephrine from rat isolated trachea. Am Rev Respir Dis 146:1182-1186.

Rahal S, McVeigh LI, Zhang Y, Guan Y, Breyer MD, and Kennedy CR (2006) Increased severity of renal impairment in nephritic mice lacking the $\mathrm{EP}_{1}$ receptor. Can J Physiol Pharmacol 84:877-885.

Rampton DS and Collins CE (1993) Review article: thromboxanes in inflammatory bowel disease-pathogenic and therapeutic implications. Aliment Pharmacol Ther 7:357-367.

Randall MJ, Parry MJ, Hawkeswood E, Cross PE, and Dickinson RP (1981) UK37,248 , a novel, selective thromboxane synthetase inhibitor with platelet antiaggregatory and anti-thrombotic activity. Thromb Res 23:145-162.

Rangachari PK and Betti PA (1993) Biological activity of metabolites of PGD2 on canine proximal colon. Am J Physiol 264:G886-G894.

Rangachari PK, Betti PA, Prior ET, and Roberts LJ 2nd (1995) Effects of a selective DP receptors agonist (BW 245C) and antagonist (BW A868C) on the canine colonic epithelium: an argument for a different DP receptor. J Pharmacol Exp Ther 275:611-617.

Rao R, Redha R, Macias-Perez I, Su Y, Hao C, Zent R, Breyer MD, and Pozzi A (2007) Prostaglandin E2-EP4 receptor promotes endothelial cell migration via ERK activation and angiogenesis in vivo. $J$ Biol Chem 282:16959-16968.

Raychowdhury MK, Yukawa M, Collins LJ, McGrail SH, Kent KC, and Ware JA (1994) Alternative splicing produces a divergent cytoplasmic tail in the human endothelial thromboxane $A_{2}$ receptors. J Biol Chem 269:19256-19261.

Regan JW (2003) $\mathrm{EP}_{2}$ and $\mathrm{EP}_{4}$ prostanoid receptor signaling. Life Sci 74:143-153.

Regan JW, Bailey TJ, Donello JE, Pierce KL, Pepperl DJ, Zhang D, Kedzie KM, Fairbairn CE, Bogardus AM, Woodward DF, et al. (1994a) Molecular cloning and expression of human $\mathrm{EP}_{3}$ receptors: evidence of three variants with differing carboxyl termini. Br J Pharmacol 112:377-385.

Regan JW, Bailey TJ, Pepperl DJ, Pierce KL, Bogardus AM, Donello JE, Fairbairn CE, Kedzie KM, Woodward DF, and Gil DW (1994b) Cloning of a novel human prostaglandin receptor with characteristics of pharmacologically defined $\mathrm{EP}_{2}$ subtype. Mol Pharmacol 46:213-220.

Reid HM and Kinsella BT (2007) Palmitoylation of the $\mathrm{TP}_{\beta}$ isoform of the human thromboxane $\mathrm{A}_{2}$ receptor. Modulation of $\mathrm{G}$ protein: effector coupling and modes of receptor internalization. Cell Signal 19:1056-1070.

Reinold H, Ahmadi S, Depner UB, Layh B, Heindl C, Hamza M, Pahl A, Brune K, Narumiya S, Müller U, et al. (2005) Spinal inflammatory hyperalgesia is mediated by prostaglandin $\mathrm{E}$ receptors of the $\mathrm{EP}_{2}$ subtype. J Clin Invest 115:673-679.

Richards JA and Brueggemeier RW (2003) Prostaglandin $\mathrm{E}_{2}$ regulates aromatase activity and expression in human adipose stromal cells via two distinct receptor subtypes. J Clin Endocrinol Metab 88:2810-2816.

Richter M, Krauss AH, Woodward DF, and Lütjen-Drecoll E (2003) Morphological changes in the anterior eye segment after long term treatment with different selective prostaglandin agonists and a prostamide. Invest Ophthalmol Vis Sci 44:4419-4426.

Ritchie RH, Rosenkranz AC, Huynh LP, Stephenson T, Kaye DM, and Dusting GJ (2004) Activation of IP receptors prevents cardiomyocyte hypertrophy via cAMPdependent signaling. Am J Physiol Heart Circ Physiol 287:H1179-H1185.

Robertson FM, Simeone AM, Mazumdar A, Shah AH, McMurray JS, Ghosh S, and Cristofanilli M (2008) Molecular and pharmacological blockade of the EP4 receptor selectively inhibits both proliferation and invasion of human inflammatory breast cancer cells. J Exp Ther Oncol 7:299-312.

Rocha PN, Plumb TJ, Robinson LA, Spurney R, Pisetsky D, Koller BH, and Coffman TM (2005) Role of thromboxane $\mathrm{A}_{2}$ in the induction of apoptosis of immature thymocytes by lipopolysaccharide. Clin Diagn Lab Immunol 12:896-903.

Rolin S, Masereel B, and Dogné JM (2006) Prostanoids as pharmacological targets in COPD and asthma. Eur J Pharmacol 533:89-100.

Rowlands DK, Kao C, and Wise H (2001) Regulation of prostacyclin and prostaglandin $\mathrm{E}(2)$ receptor mediated responses in adult rat dorsal root ganglion cells, in vitro. Br J Pharmacol 133:13-22.

Roy SJ, Parent A, Gallant MA, de Brum-Fernandes AJ, Stanková J, and Parent JL (2010) Characterization of C-terminal determinants involved in CRTH2 receptor trafficking: identification of a recycling motif. Eur J Pharmacol 630:10-18.

Rudic RD, Brinster D, Cheng Y, Fries S, Song WL, Austin S, Coffman TM, and FitzGerald GA (2005) COX-2-derived prostacyclin modulates vascular remodeling. Circ Res 96:1240-1247.

Ruel R, Lacombe P, Abramovitz M, Godbout C, Lamontagne S, Rochette C, Sawyer N, Stocco R, Tremblay NM, Metters KM, et al. (1999) New class of biphenylene dibenzazocinones as potent ligands for the human $\mathrm{EP}_{1}$ prostanoid receptor. Bioorg Med Chem Lett 9:2699-2704.

Rutkai I, Feher A, Erdei N, Henrion D, Papp Z, Edes I, Koller A, Kaley G, and Bagi $\mathrm{Z}$ (2009) Activation of prostaglandin $\mathrm{E}_{2} \mathrm{EP}_{1}$ receptor increases arteriolar tone and blood pressure in mice with type 2 diabetes. Cardiovasc Res 83:148-154.

Saeki T, Ota T, Aihara M, and Araie M (2009) Effects of prostanoid EP agonists on mouse intraocular pressure. Invest Ophthalmol Vis Sci 50:2201-2208.

Sagana RL, Yan M, Cornett AM, Tsui JL, Stephenson DA, Huang SK, Moore BB, Ballinger MN, Melonakos J, Kontos CD, et al. (2009) Phosphatase and tensin homologue on chromosome 10 (PTEN) directs prostaglandin $\mathrm{E}_{2}$-mediated fibroblast responses via regulation of $\mathrm{E}$ prostanoid 2 receptor expression. $J$ Biol Chem 284:32264-32271.

Sagara T, Gaton DD, Lindsey JD, Gabelt BT, Kaufman PL, and Weinreb RN (1999) Topical prostaglandin $\mathrm{F}_{2 \alpha}$ treatment reduces collagen types I, III, and IV in the monkey uveoscleral outflow pathway. Arch Ophthalmol 117:794-801.

Saito M, Tanaka H, Sasaki M, Kurose H, and Nakahata N (2010) Involvement of aquaporin in thromboxane $\mathrm{A}_{2}$ receptor-mediated $\mathrm{G}_{12 / 13} / \mathrm{RhoA} / \mathrm{NHE}$-sensitive cell swelling in 1321N1 human astrocytoma cells. Cell Signal 22:41-46.

Saito O, Guan Y, Qi Z, Davis LS, Kömhoff M, Sugimoto Y, Narumiya S, Breyer RM, and Breyer MD (2003) Expression of the prostaglandin F receptor (FP) gene along the mouse genitourinary tract. Am J Physiol Renal Physiol 284:F1164-F1170.

Sakamoto A, Matsumura J, Mii S, Gotoh Y, and Ogawa R (2004) A prostaglandin E2 receptor subtype EP4 agonist attenuates cardiovascular depression in endotoxin shock by inhibiting inflammatory cytokines and nitric oxide production. Shock 22:76-81.

Sakuma Y, Tanaka K, Suda M, Yasoda A, Natsui K, Tanaka I, Ushikubi F, Narumiya S, Segi E, Sugimoto Y, et al. (2000) Crucial involvement of the EP4 subtype of prostaglandin E receptor in osteoclast formation by proinflammatory cytokine and lipopolysaccharide. J Bone Miner Res 15:218-227.

Saleem S, Ahmad AS, Maruyama T, Narumiya S, and Doré S (2009a) PGF $_{2 \alpha}$ FP receptor contributes to brain damage following transient focal brain ischemia. Neurotox Res 15:62-70.

Saleem S, Kim YT, Maruyama T, Narumiya S, and Doré S (2009b) Reduced acute brain injury in $\mathrm{PGE}_{2} \mathrm{EP}_{3}$ receptor-deficient mice after cerebral ischemia. J Neuroimmunol 208:87-93.

Saleem S, Li RC, Wei G, and Doré S (2007a) Effects of $\mathrm{EP}_{1}$ receptor on cerebral blood flow in the middle cerebral artery occlusion model of stroke in mice. J Neurosci Res 85:2433-2440.

Saleem S, Zhuang H, de Brum-Fernandes AJ, Maruyama T, Narumiya S, and Doré $\mathrm{S}(2007 \mathrm{~b}) \mathrm{PGD}(2) \mathrm{DP}_{1}$ receptor protects brain from ischemia-reperfusion injury Eur J Neurosci 26:73-78.

Sales KJ, Boddy SC, and Jabbour HN (2008) F-prostanoid receptor alters adhesion, morphology and migration of endometrial adenocarcinoma cells. Oncogene 27: 2466-2477.

Sales KJ, Katz AA, Davis M, Hinz S, Soeters RP, Hofmeyr MD, Millar RP, and Jabbour HN (2001) Cyclooxygenase-2 expression and prostaglandin E(2) synthesis are up-regulated in carcinomas of the cervix: a possible autocrine/paracrine regulation of neoplastic cell function via $\mathrm{EP}_{2} / \mathrm{EP}_{4}$ receptors. J Clin Endocrinol Metab 86:2243-2249

Sales KJ, List T, Boddy SC, Williams AR, Anderson RA, Naor Z, and Jabbour HN (2005) A novel angiogenic role for prostaglandin $\mathrm{F}_{2 \alpha}$-FP receptor interaction in human endometrial adenocarcinomas. Cancer Res 65:7707-7716.

Sales KJ, Maldonado-Pérez D, Grant V, Catalano RD, Wilson MR, Brown P, Williams AR, Anderson RA, Thompson EA, and Jabbour HN (2009) Prostaglandin $\mathrm{F}_{2 \alpha}$-F-prostanoid receptor regulates CXCL8 expression in endometrial adenocarcinoma cells via the calcium-calcineurin-NFAT pathway. Biochim Biophys Acto 1793:1917-1928.

Sales KJ, Milne SA, Williams AR, Anderson RA, and Jabbour HN (2004) Expression, localization, and signaling of prostaglandin $\mathrm{F}_{2 \alpha}$ receptor in human endometria adenocarcinoma: regulation of proliferation by activation of the epidermal growth factor receptor and mitogen-activated protein kinase signaling pathways. $J$ Clin Endocrinol Metab 89:986-993.

Salzman PM, Salmon JA, and Moncada S (1980) Prostacyclin and thromboxane $\mathrm{A}_{2}$ synthesis by rabbit pulmonary artery. J Pharmacol Exp Ther 215:240-247.

Sanchez T and Moreno JJ (2006) GR 63799X, an $\mathrm{EP}_{3}$ receptor agonist, induced S phase arrest and 3T6 fibroblast growth inhibition. Eur $J$ Pharmacol 529:16-23.

Sanchez-Alavez M, Klein I, Brownell SE, Tabarean IV, Davis CN, Conti B, and Bartfai T (2007) Night eating and obesity in the EP3R-deficient mouse. Proc Nat Acad Sci USA 104:3009-3014.

Sanchez-Ramos L, Kaunitz AM, Wears RL, Delke I, and Gaudier FL (1997) Misoprostol for cervical ripening and labor induction; a meta-analysis. Obstet Gynecol 89:633-642

Sands WA and Palmer TM (2008) Regulating gene transcription in response to cyclic AMP elevation. Cell Signal 20:460-466.

Sang N, Zhang J, Marcheselli V, Bazan NG, and Chen C (2005) Postsynaptically synthesized prostaglandin $\mathrm{E}_{2}\left(\mathrm{PGE}_{2}\right)$ modulates hippocampal synaptic transmission via a presynaptic $\mathrm{PGE}_{2} \mathrm{EP}_{2}$ receptor. J Neurosci 25:9858-9870.

Sanner, JH (1969) Antagonism of prostaglandin $\mathrm{E}_{2}$ by 1-acetyl-2-(8-chloro-10,11dihydrobibenz $(b, f)(1,4)$ oxazepine-10-carbonyl) hydrazine (SC-19220). Arch Int Pharmacodyn Ther 180:46-56.

Sarkar S, Hobson AR, Hughes A, Growcott J, Woolf CJ, Thompson DG, and Aziz Q (2003) The prostaglandin $\mathrm{E}_{2}$ receptor-1 (EP-1) mediates acid-induced visceral pain hypersensitivity in human. Gastroenterology 124:18-25.

Sarrazin P, Bkaily G, Haché R, Patry C, Dumais R, Rocha FA, and de BrumFernandes AJ (2001) Characterization of the prostaglandin receptors in human osteoblasts in culture. Prostaglandins Leukot Essent Fatty Acids 64:203-210.

Satarug S, Wisedpanichkij R, Takeda K, Li B, Na-Bangchang K, Moore MR, and Shibahara S (2008) Prostaglandin $\mathrm{D}_{2}$ induces heme oxygenase-1 mRNA expression through the $\mathrm{DP}_{2}$ receptor. Biochem Biophys Res Commun 377:878-883.

Satoh T, Moroi R, Aritake K, Urade Y, Kanai Y, Sumi K, Yokozeki H, Hirai H Nagata K, Hara T, et al. (2006) Prostaglandin D2 plays an essential role in chronic allergic inflammation of the skin via CRTH2 receptor. J Immunol 177:2621-2629.

Savage MA, Moummi C, Karabatsos PJ, and Lanthorn TH (1993) SC-46275: a potent and highly selective agonist at the EP3 receptor. Prostaglandins Leukot Essent Fatty Acids 49:939-943.

Savonenko A, Munoz P, Melnikova T, Wang Q, Liang X, Breyer RM, Montine TJ, Kirkwood A, and Andreasson K (2009) Impaired cognition, sensorimotor gating, and hippocampal long-term depression in mice lacking the prostaglandin $\mathrm{E}_{2} \mathrm{EP}_{2}$ receptor. Exp Neurol 217:63-73.

Sawyer N, Cauchon E, Chateauneuf A, Cruz RP, Nicholson DW, Metters KM, O'Neill GP, and Gervais FG (2002) Molecular pharmacology of the human prostaglandin $\mathrm{D}_{2}$ receptor, CRTH2. Br J Pharmacol 137:1163-1172.

Scandella E, Men Y, Gillessen S, Förster R, and Groettrup M (2002) Prostaglandin E2 is a key factor for CCR7 surface expression and migration of monocyte-derived dendritic cells. Blood 100:1354-1361.

Schaafsma D, Gosens R, Bos IS, Meurs H, Zaagsma J, and Nelemans SA (2005) Role of contractile prostaglandins and Rho-kinase in growth factor-induced airway smooth muscle contraction. Respir Res 6:85.

Schafer AI, Cooper B, O'Hara D, and Handin RI (1979) Identification of platelet receptors for prostaglandin $\mathrm{I}_{2}$ and $\mathrm{D}_{2}$. J Biol Chem 254:2914-2917. 
Schillinger E, Prior G, Speckenbach A, and Wellershoff S (1979) Receptor binding in various tissues of $\mathrm{PGE}_{2}, \mathrm{PGF}_{2 \alpha}$ and sulprostone, a novel $\mathrm{PGE}_{2}$ derivative. Prostaglandins 18:293-302.

Schlicker E and Marr I (1997) Mutual interactions of the presynaptic histamine H3 and prostaglandin $\mathrm{EP}_{3}$ receptors on the noradrenergic terminals in the mouse brain. Neuroscience 79:247-254.

Schlötzer-Schrehardt U, Zenkel M, and Nüsing RM (2002) Expression and localization of FP and EP prostanoid receptor subtypes in human ocular tissues. Invest Ophthalmol Vis Sci 43:1475-1487.

Schmid A, Thierauch KH, Schleuning WD, and Dinter H (1995) Splice variants of the human $\mathrm{EP}_{3}$ receptor for prostaglandin $\mathrm{E}_{2}$. Eur J Biochem 228:23-30.

Schmitz T, Dallot E, Leroy MJ, Breuiller-Fouché M, Ferré F, and Cabrol D (2001) $\mathrm{EP} 4$ receptors mediate prostaglandin $\mathrm{E}_{2}$-stimulated glycosaminoglycan synthesis in human cervical fibroblasts in culture. Mol Hum Reprod 7:397-402.

Schneider A, Guan Y, Zhang Y, Magnuson MA, Pettepher C, Loftin CD, Langenbach R, Breyer RM, and Breyer MD (2004) Generation of a conditional allele of the mouse prostaglandin EP4 receptor. Genesis 40:7-14.

Schratl P, Royer JF, Kostenis E, Ulven T, Sturm EM, Waldhoer M, Hoefler G, Schuligoi R, Lippe IT, Peskar BA, et al. (2007) The role of the prostaglandin $\mathrm{D}_{2}$ receptor, DP, in eosinophil trafficking. J Immunol 179:4792-4799.

Schröder A, Newgreen D, and Andersson KE (2004) Detrusor responses to prostaglandin $\mathrm{E}_{2}$ and bladder outlet obstruction in wild-type and $\mathrm{EP}_{1}$ receptor knockout mice. J Urol 172:1166-1170.

Schweda F, Klar J, Narumiya S, Nüsing RM, and Kurtz A (2004) Stimulation of renin release by prostaglandin $\mathrm{E}_{2}$ is mediated by $\mathrm{EP}_{2}$ and $\mathrm{EP}_{4}$ receptors in mouse kidneys. Am J Physiol Renal Physiol 287:F427-F433.

Scott G, Jacobs S, Leopardi S, Anthony FA, Learn D, Malaviya R, and Pentland A (2005) Effects of $\mathrm{PGF}_{2 \alpha}$ on human melanocytes and regulation of the $\mathrm{FP}$ receptor in the ultraviolet radiation. Exp Cell Res 304:407-416.

Sebeková K, Eifert T, Klassen A, Heidland A, and Amann K (2007) Renal effects of S18886 (Terutroban), a TP receptor antagonist, in an experimental model of type 2 diabetes. Diabetes 56:968-974.

Segi E, Sugimoto Y, Yamasaki A, Aze Y, Oida H, Nishimura T, Murata T, Matsuoka T, Ushikubi F, Hirose M, et al. (1998) Patent ductus arteriosus and neonatal death in prostaglandin receptor EP4-deficient mice. Biochem Biophys Res Commun 246:7-12.

Seiler S, Brassard CL, and Federici ME (1990) SQ-27986 inhibition of platelet aggregation in mediated through activation of platelet prostaglandin $\mathrm{D}_{2}$ receptors. Prostaglandins 40:119-130.

Seiler SM, Brassard CL, Federici ME, Romine J, and Meanwell NA (1997) [3-[4-(4,5Diphenyl-2-oxazolyl)-5-oxazolyl]phenoxy]acetic acid (BMY 45778) is a potent nonprostanoid prostacyclin partial agonist: effects on platelet aggregation, adenylyl cyclase, cAMP levels, protein kinase, and iloprost binding. Prostaglandins 53:21-35.

Senior J, Marshall K, Sangha R, and Clayton JK (1993) In vitro characterization of prostanoid receptors on human myometrium at term pregnancy. $\mathrm{Br} J$ Pharmacol 108:501-506.

Senior J, Marshall K, Sangha R, Baxter GS, and Clayton JK (1991) In vitro characterization of prostanoid EP-receptors in the non-pregnant human myometrium. Br J Pharmacol 102:747-753.

Senior J, Sangha R, Baxter GS, Marshall K, and Clayton JK (1992) In vitro characterization of prostanoid FP-, DP-, IP- and TP-receptors on the non-pregnant human myometrium. Br J Pharmacol 107:215-221.

Seno H, Oshima M, Ishikawa TO, Oshima H, Takaku K, Chiba T, Narumiya S, and Taketo MM (2002) Cyclooxygenase-2- and prostaglandin $\mathrm{EP}(2)$ receptor $\mathrm{EP}(2)$ dependent angiogenesis in Apc(Delta716) mouse intestinal polyps. Cancer Res 62:506-511.

Serrero G and Lepak NM (1997) Prostaglandin $\mathrm{F}_{2 \alpha}$ receptor (FP receptor) agonists are potent adipose differentiation inhibitors for primary culture of adipocyte precursors in defined medium. Biochem Biophys Res Commun 233:200-202.

Sessa WC, Halushka PV, Okwu A, and Nasjletti A (1990) Characterization of the vascular thromboxane $\mathrm{A}_{2}$ /prostaglandin endoperoxide receptor in rabbit aorta. Regulation by dexamethasone. Circ Res 67:1562-1569.

Sharif NA, Crider JY, and Davis TL (2000) AL-3138 antagonizes FP prostanoid receptor-mediated inositol phosphates generation: comparison with some purported FP antagonists. J Pharm Pharmacol 52:1529-1539.

Sharif NA, Crider JY, Xu SX, and Williams GW (2000) Affinities, selectivities, potencies, and intrinsic activities of natural and synthetic prostanoids using endogenous receptors: focus on DP class prostanoids. J Pharmacol Exp Ther 293: 321-328.

Sharif NA and Davis TL (2002) Cloned human $\mathrm{EP}_{1}$ prostanoid receptor pharmacology characterized using radioligand binding techniques. J Pharm Pharmacol 54:539-547.

Sharif NA, McLaughlin MA, Kelly CR, Xu S, Crider JY, Williams GW, and Parker JL (2006) Preclinical pharmacology of AL-12182, a new ocular hypotensive 11-oxa prostaglandin analog. J Ocul Pharmacol Ther 22:291-309.

Sharif NA, Williams GW, Crider JY, Xu SX, and Davis TL (2004) Molecular pharmacology of the $\mathrm{DP} / \mathrm{EP}_{2}$ class prostaglandin AL-6598 and quantitative autoradiographic visualization of $\mathrm{DP}$ and $\mathrm{EP}_{2}$ receptor sites in human eyes. J Ocul Pharmacol Ther 20:489-508.

Sheller JR, Mitchell D, Meyrick B, Oates J, and Breyer R (2000) EP(2) receptor mediates bronchodilation by PGE(2) in mice. $J$ Appl Physiol 88:2214-2218.

Shie FS, Montine KS, Breyer RM, and Montine TJ (2005) Microglial EP2 is critical to neurotoxicity from activated cerebral innate immunity. Glia 52:70-77.

Shichijo M, Arimura A, Hirano Y, Yasui K, Suzuki N, Deguchi M, and Abraham WM (2009) A prostaglandin D2 receptor antagonist modifies experimental asthma in sheep. Clin Exp Allergy 39:1404-1414.

Shimazaki Y, Kameo K, Tanami T, Tanaka H, Ono N, Kiuchi Y, Okamoto S, Sato F and Ichikawa A (2000) Synthesis and structure-activity relationships of a new class of selective $\mathrm{EP}_{3}$ receptor agonist, 13,14-didehydro-16-phenoxy analogues of prostaglandin $\mathrm{E}_{1}$. Bioorg Med Chem 8:353-362.

Shiraishi Y, Asano K, Nakajima T, Oguma T, Suzuki Y, Shiomi T, Sayama K, Niimi
K, Wakaki M, Kagyo J, et al. (2005) Prostaglandin $D_{2}$-induced eosinophilic airway inflammation is mediated by CRTH2 receptor. J Pharmacol Exp Ther 312:954960.

Shiraishi Y, Asano K, Niimi K, Fukunaga K, Wakaki M, Kagyo J, Takihara T, Ueda S, Nakajima T, Oguma T, et al. (2008) Cyclooxygenase-2/prostaglandin $\mathrm{D}_{2} / \mathrm{CRTH} 2$ pathway mediates double-stranded RNA-induced enhancement of allergic airway inflammation. J Immunol 180:541-549.

Shoji Y, Takahashi M, Kitamura T, Watanabe K, Kawamori T, Maruyama T, Sugimoto Y, Negishi M, Narumiya S, Sugimura T, et al. (2004) Downregulation of prostaglandin E receptor subtype EP3 during colon cancer development. Gut 53:1151-1158.

Shoji Y, Takahashi M, Takasuka N, Niho N, Kitamura T, Sato H, Maruyama T, Sugimoto Y, Narumiya S, Sugimura T, et al. (2005) Prostaglandin E receptor EP3 deficiency modifies tumor outcome in mouse two-stage skin carcinogenesis. Car cinogenesis 26:2116-2122.

Shum WW, Le GY, Jones RL, Gurney AM, and Sasaki Y (2003) Involvement of Rho-kinase in contraction of guinea-pig aorta induced by prostanoid $\mathrm{EP}_{3}$ receptor agonists. Br J Pharmacol 139:1449-1461.

Singh J, Zeller W, Zhou N, Hategen G, Mishra R, Polozov A, Yu P, Onua E, Zhang $\mathrm{J}$, Zembower D, et al. (2009) Antagonists of the $\mathrm{EP}_{3}$ receptor for prostaglandin $\mathrm{E}$ are novel antiplatelet agents that do not prolong bleeding. ACS Chem Biol 4:115126.

Smid SD and Svensson KM (2009) Inhibition of cyclooxygenase-2 and $\mathrm{EP}_{1}$ receptor antagonism reduces human colonic longitudinal muscle contractility in vitro. Prostaglandins Other Lipid Mediat 88:117-121.

Smith JA, Amagasu SM, Eglen RM, Hunter JC, and Bley KR (1998) Characterization of prostanoid receptor-evoked responses in rat sensory neurones. $B r J P h a r$ macol 124:513-523.

Smith OP, Battersby S, Sales KJ, Critchley HO, and Jabbour HN (2006) Prostacyclin receptor up-regulates the expression of angiogenic genes in human endometrium via cross talk with epidermal growth factor receptor and the extracellular signaling receptor kinase 1/2 pathway. Endocrinology 147:1697-1705.

Smith RL, Lee T, Gould NP, and Cragoe EJ Jr (1977) Prostaglandin isosteres 1. (8-Aza-, 8,10-diaza, and 8-aza-11-thia)-9-oxoprostanoic acids and their derivatives. J Med Chem 20:1292-1299.

Smith-Thomas L, Moustafa M, Spada CS, Shi L, Dawson RA, Wagner M, Balafa C Kedzie KM, Reagan JW, Krauss AH, et al. (2004) Latanoprost-induced pigmentation in human iridial melanocytes is fibroblast dependent. Exp Eye Res 78:973985

Smock SL, Pan LC, Castleberry TA, Lu B, Mather RJ, and Owen TA (1999) Cloning, structural characterization, and chromosomal localization of the gene encoding the human prostaglandin $\mathrm{E}_{2}$ receptor $\mathrm{EP}_{2}$ subtype. Gene 237:393-402.

Song P, Zhang M, Wang S, Xu J, Choi HC, and Zou MH (2009) Thromboxane A receptor activates a Rho-associated kinase/LKB1/PTEN pathway to attenuat endothelium insulin signaling. J Biol Chem 284:17120-17128.

Sonnenburg WK, Zhu JH, and Smith WL (1990) A prostaglandin E receptor coupled to a pertussis toxin-sensitive guanine nucleotide regulatory protein in rabbit cortical collecting tubule cells. J Biol Chem 265:8479-8483.

Sonoshita M, Takaku K, Sasaki N, Sugimoto Y, Ushikubi F, Narumiya S, Oshima M and Taketo MM (2001) Acceleration of intestinal polyposis through prostaglandin receptor $\mathrm{EP}_{2}$ in $\mathrm{APC}($ Delta 716) knockout mice. Nature Med 7:1048-1051.

Sooranna SR, Grigsby P, Myatt L, Bennett PR, and Johnson MR (2005) Prostanoid receptors in human uterine myocytes: the effect of reproductive state and stretch. Mol Hum Reprod 11:859-864.

Southall MD and Vasko MR (2001) Prostaglandin receptor subtypes, EP3C and EP4, mediate the prostaglandin $\mathrm{E}_{2}$-induced cAMP production and sensitization of sensory neurons. J Biol Chem 276:16083-16091.

Soyka R, Heckel A, Nickl J, Eisert W, Müller TH, and Weisenberger H (1994) 6,6-Disubstituted hex-5-enoic acid derivatives as combined thromboxane $\mathrm{A}_{2}$ receptor antagonists and synthase inhibitors. J Med Chem 37:26-39.

Spada CS, Krauss AH, Woodward DF, Chen J, Protzman CE, Nieves AL, Wheeler LA, Scott DF, and Sachs G (2005) Bimatoprost and prostaglandin F2 $\alpha$ selectively stimulate intracellular calcium signaling in different cat iris sphincter cells. Exp Eye Res 80:135-145.

Spik I, Brénuchon C, Angéli V, Staumont D, Fleury S, Capron M, Trottein F, and Dombrowicz D (2005) Activation of the prostaglandin $\mathrm{D}_{2}$ receptor $\mathrm{DP}_{2} / \mathrm{CRTH} 2$ increases allergic inflammation in mouse. J Immunol 174:3703-3708.

Spilman CH, Bergstrom KK, and Forbes AD (1977) Effect of 19-hydroxyprostaglandins on oviductal and uterine motility. Prostaglandins 13:795-805.

Spinella F, Rosanò L, Di Castro V, Natali PG, and Bagnato A (2004) Endothelin-1induced prostaglandin $\mathrm{E}_{2}-\mathrm{EP}_{2}, \mathrm{EP}_{4}$ signaling regulates vascular endothelia growth factor production and ovarian carcinoma cell invasion. J Biol Chem $\mathbf{2 7 9}$ 46700-46705.

Sprague PW, Heikes JE, Gougoutas JZ, Malley MF, Harris DN, and Greenberg R (1985) Synthesis and in vitro pharmacology of 7-oxabicyclo[2.2.1]heptane analogues of thromboxane $\mathrm{A}_{2} / \mathrm{PGH}_{2}$. J Med Chem 28:1580-1590.

Stamer WD, Piwnica D, Jolas T, Carling RW, Cornell CL, Fliri H, Martos J, Pettit SN, Wang JW, and Woodward DF (2010) Cellular basis for bimatoprost effects on human conventional outflow. Invest Ophthalmol Vis Sci 51:5176-5181.

Stearns BA, Baccei C, Bain G, Broadhead A, Clark RC, Coate H, Evans JF, Fagan P Hutchinson JH, King C, et al. (2009) Novel tricyclic antagonists of the prostaglandin $\mathrm{D}_{2}$ receptor $\mathrm{DP}_{2}$ with efficacy in a murine model of allergic rhinitis. Bioorg Med Chem Lett 19:4647-4651.

Stebbins KJ, Broadhead AR, Baccei CS, Scott JM, Truong YP, Coate H, Stock NS, Santini AM, Fagan P, Prodanovich P, et al. (2010) Pharmacological blockade of the $\mathrm{DP}_{2}$ receptor inhibits cigarette smoke-induced inflammation, mucus cell metaplasia, and epithelial hyperplasia in the mouse lung. J Pharmacol Exp Ther 332:764775 .

Stjernschantz JW (2001) From PGF P $_{2 \alpha}$-isopropyl ester to latanoprost: a review of the development of xalatan: the Proctor Lecture. Invest Ophthalmol Vis Sci 42:11341145 . 
Stock JL, Shinjo K, Burkhardt J, Roach M, Taniguchi K, Ishikawa T, Kim HS, Flannery PJ, Coffman TM, McNeish JD, et al. (2001) The prostaglandin $\mathrm{E}_{2} \mathrm{EP}_{1}$ receptor mediates pain perception and regulates blood pressure. $J$ Clin Invest 107:325-331.

Stubbs VE, Schratl P, Hartnell A, Williams TJ, Peskar BA, Heinemann A, and Sabroe I (2002) Indomethacin causes prostaglandin $\mathrm{D}_{2}$-like and eotaxin-like selective responses in eosinophils and basophils. J Biol Chem 277:26012-26020.

Sturino CF, O’Neill G, Lachance N, Boyd M, Berthelette C, Labelle M, Li L, Roy B, Scheigetz J, Tsou N, et al. (2007) Discovery of a potent and selective prostaglandin $\mathrm{D}_{2}$ receptor antagonist, [(3R)-4-(4-chloro-benzyl)-7-fluoro-5-(methylsulfonyl)1,2,3,4-tetrahydrocyclopenta[b] indol-3-yl]-acetic acid (MK-0524). J Med Chem 50:794-806.

Sturm EM, Schratl P, Schuligoi R, Konya V, Sturm GJ, Lippe IT, Peskar BA, and Heinemann A (2008) Prostaglandin $\mathrm{E}_{2}$ inhibits eosinophil trafficking through E-prostanoid 2 receptors. J Immunol 181:7273-7283.

Su X, Lashinger ES, Leon LA, Hoffman BE, Hieble JP, Gardner SD, Fries HE, Edwards RM, Li J, and Laping NJ (2008a) An excitatory role for peripheral $\mathrm{EP}_{3}$ receptors in bladder afferent function. Am J Physiol Renal Physiol 295:F585F594

Su X, Leon LA, Wu CW, Morrow DM, Jaworski JP, Hieble JP, Lashinger ES, Jin J, Edwards RM, and Laping NJ (2008b) Modulation of bladder function by prostaglandin $\mathrm{EP}_{3}$ receptors in the central nervous system. Am J Physiol Renal Physiol 295:F984-F994.

Subbaramaiah K, Hudis C, Chang SH, Hla T, and Dannenberg AJ (2008) $\mathrm{EP}_{2}$ and $\mathrm{EP}_{4}$ receptors regulate aromatase expression in human adipocytes and breast cancer cells. Evidence of a BRCA1 and p300 exchange. J Biol Chem 283:34333444

Suganami T, Mori K, Tanaka I, Mukoyama M, Sugawara A, Makino H, Muro S, Yahata K, Ohuchida S, Maruyama T, et al. (2003) Role of prostaglandin E receptor $\mathrm{EP}_{1}$ subtype in the development of renal injury in genetically hypertensive rats. Hypertension 42:1183-1190.

Sugimoto H, Shichijo M, Iino T, Manabe Y, Watanabe A, Shimazaki M, Gantner F, and Bacon KB (2003) An orally bioavailable small molecule antagonist of CRTH2, ramatroban (BAY U3405), inhibits prostaglandin $\mathrm{D}_{2}$-induced eosinophil migration in vitro. J Pharmacol Exp Ther 305:347-352.

Sugimoto M, Arai I, Futaki N, Hashimoto Y, Sakurai T, Honma Y, and Nakaike S (2007) The anti-pruritic efficacy of TS-022, a prostanoid $\mathrm{DP}_{1}$ receptor agonist, is dependent on the endogenous prostaglandin $\mathrm{D}_{2}$ level in the skin of NC/Nga mice. Eur $J$ Pharmacol 564:196-203.

Sugimoto Y, Fukada Y, Mori D, Tanaka S, Yamane H, Okuno Y, Deai K, Tsuchiya S, Tsujimoto G, and Ichikawa A (2005) Prostaglandin $\mathrm{E}_{2}$ stimulates granulocyte colony-stimulating factor production via the prostanoid $\mathrm{EP}_{2}$ receptor in mouse peritoneal neutrophils. J Immunol 175:2606-2612.

Sugimoto Y, Hasumoto K, Namba T, Irie A, Katsuyama M, Negishi M, Kakizuka A, Narumiya S, and Ichikawa A (1994a) Cloning and expression of a cDNA for mouse prostaglandin F receptor. J Biol Chem 269:1356-1360.

Sugimoto Y, Namba T, Honda A, Hayashi Y, Negishi M, Ichikawa A, and Narumiya $\mathrm{S}$ (1992) Cloning and expression of a cDNA for mouse prostaglandin $\mathrm{E}$ receptor $\mathrm{EP}_{3}$ subtype. J Biol Chem 267:6463-6466.

Sugimoto Y, Negishi M, Hayashi Y, Namba T, Honda A, Watabe A, Hirata M, Narumiya S, and Ichikawa A (1993) Two isoforms of the $\mathrm{EP}_{3}$ receptor with different carboxyl-terminal domains. Identical ligand binding properties and different coupling properties with Gi proteins. J Biol Chem 268:2712-2718.

Sugimoto Y, Shigemoto R, Namba T, Negishi M, Mizuno N, Narumiya S, and Ichikawa A (1994b) Distribution of the messenger RNA for the prostaglandin E receptor subtype $\mathrm{EP}_{3}$ in the mouse nervous system. Neuroscience 62:919-928.

Sugimoto Y, Yamasaki A, Segi E, Tsuboi K, Aze Y, Nishimura T, Oida H, Yoshida N, Tanaka T, Katsuyama M, et al. (1997) Failure of parturition in mice lacking the prostaglandin F receptor. Science 277:681-683.

Sugiura H, Liu X, Togo S, Kobayashi T, Shen L, Kawasaki S, Kamio K, Wang XQ Mao LJ, and Rennard SI (2007) Prostaglandin $\mathrm{E}_{2}$ protects human lung fibroblasts from cigarette smoke extract-induced apoptosis via $\mathrm{EP}_{2}$ receptor activation. J Cell Physiol 210:99-110.

Sung YM, He G, and Fischer SM (2005) Lack of expression of the EP2 but not EP3 receptor for prostaglandin E2 results in suppression of skin tumor development. Cancer Res 65:9304-9311.

Sung YM, He G, Hwang DH, and Fischer SM (2006) Overexpression of the prostaglandin $\mathrm{E}_{2}$ receptor $\mathrm{EP}_{2}$ results in enhanced skin tumor development. Oncogene 25:5507-5516.

Suzawa T, Miyaura C, Inada M, Maruyama T, Sugimoto Y, Ushikubi F, Ichikawa A, Narumiya S, and Suda T (2000) The role of prostaglandin E receptor subtypes $\left(\mathrm{EP}_{1}, \mathrm{EP}_{2}, \mathrm{EP}_{3}\right.$, and $\left.\mathrm{EP}_{4}\right)$ in bone resorption: an analysis using specific agonists for the respective EPs. Endocrinology 141:1554-1559.

Suzuki-Yamamoto T, Toida K, Sugimoto Y, and Ishimura K (2009) Colocalization of prostaglandin F2 alpha receptor FP and prostaglandin F synthase-I in the spinal cord. J Lipid Res 50:1996-2003.

Svensson J and Hamberg M (1976) Thromboxane $\mathrm{A}_{2}$ and prostaglandin $\mathrm{H}_{2}$ : potent stimulators of the swine coronary artery. Prostaglandins 12:943-950.

Swayne GT, Maguire J, Dolan J, Raval P, Dane G, Greener M, and Owen DA (1988) Evidence for homogeneity of thromboxane $\mathrm{A}_{2}$ receptor using structurally different antagonists. Eur J Pharmacol 152:311-319.

Taggart MJ, Europe-Finner GN, and Mitchell BF (2008) Possible dual roles for prostacyclin in human pregnancy and labor. J Clin Invest 118:3829-3832.

Tai H, Miyaura C, Pilbeam CC, Tamura T, Ohsugi Y, Koishihara Y, Kubodera N, Kawaguchi H, Raisz LG, and Suda T (1997) Transcriptional induction of cyclooxygenase-2 in osteoblasts is involved in interleukin-6-induced osteoclast formation. Endocrinology 138:2372-2379.

Tajima T, Murata T, Aritake K, Urade Y, Hirai H, Nakamura M, Ozaki H, and Hori M (2008) Lipopolysaccharide induces macrophage migration via prostaglandin $\mathrm{D}_{2}$ and prostaglandin $\mathrm{E}_{2}$. J Pharmacol Exp Ther 326:493-501.

Takadera T and Ohyashiki T (2006) Prostaglandin $\mathrm{E}_{2}$ deteriorates $N$-methyl-D- aspartate receptor-mediated cytotoxicity possibly by activating $\mathrm{EP}_{2}$ receptors in cultured cortical neurons, Life Sci 78:1878-1883.

Takadera T, Shiraishi Y, and Ohyashiki T (2004) Prostaglandin $\mathrm{E}_{2}$ induced caspasedependent apoptosis possibly through activation of $\mathrm{EP}_{2}$ receptors in cultured hippocampal neurons. Neurochem Int 45:713-719.

Takafuji VA, Evans A, Lynch KR, and Roche JK (2002) $\mathrm{PGE}_{2}$ receptors and synthesis in human gastric mucosa: perturbation in cancer. Prostaglandins Leukot Essent Fatty Acids 66:71-81.

Takagi Y, Nakajima T, Shimazaki A, Kageyama M, Matsugi T, Matsumura Y, Gabel BT, Kaufman PL, and Hara H (2004) Pharmacological characteristics of AF-168 (tafluprost), a new prostanoid FP receptor agonist, as an ocular hypotensive drug. Exp Eye Res 78:767-776.

Takahara K, Murray R, FitzGerald GA, and Fitzgerald DJ (1990) The response to thromboxane $\mathrm{A}_{2}$ analogues in human platelets. Discrimination of two binding sites linked to distinct effector systems. J Biol Chem 265:6836-6844.

Takahashi Y, Tokuoka S, Masuda T, Hirano Y, Nagao M, Tanaka H, Inagaki N, Narumiya S, and Nagai H (2002) Augmentation of allergic inflammation in prostanoid IP receptor deficient mice. Br J Pharmacol 137:315-322.

Takaoka A, Arai I, Sugimoto M, Futaki N, Sakurai T, Honma Y, and Nakaike S (2007) Role of scratch-induced cutaneous prostaglandin D production of atopic-like scratching behaviour in mice. Exp Derm 16:331-339.

Takasaki I, Nojima H, Shiraki K, Sugimoto Y, Ichikawa A, Ushikubi F, Narumiya S, and Kuraishi $\mathrm{Y}$ (2005) Involvement of cyclooxygenase-2 and $\mathrm{EP}_{3}$ receptor in acute herpetic but not postherpetic pain in mice. Neuropharmacology 49:283-292.

Takayama K, García-Cardena G, Sukhova GK, Comander J, Gimbrone MA Jr, and Libby $\mathrm{P}$ (2002) Prostaglandin $\mathrm{E}_{2}$ suppresses chemokine production in human macrophages through the $\mathrm{EP}_{4}$ receptor. J Biol Chem 277:44147-44154.

Takayama K, Yuhki K, Ono K, Fujino T, Hara A, Yamada T, Kuriyama S, Karibe H, Okada $\mathrm{Y}$, Takahata $\mathrm{O}$, et al. (2005) Thromboxane $\mathrm{A}_{2}$ and prostaglandin $\mathrm{F}_{2}$ mediate inflammatory tachycardia. Nature Med 11:562-566.

Take I, Kobayashi Y, Yamamoto Y, Tsuboi H, Ochi T, Uematsu S, Okafuji N Kurihara S, Udagawa N, and Takahashi N (2005) Prostaglandin E $E_{2}$ strongly inhibits human osteoclast formation. Endocrinology 146:5204-5214.

Takeuchi K, Aihara E, Sasaki Y, Nomura Y, and Ise F (2006) Involvement of cyclooxygenase-1, prostaglandin $\mathrm{E}_{2}$ and $\mathrm{EP}_{1}$ receptors in acid-induced $\mathrm{HC} 03$ secretion in stomach. J Physiol Pharmacol 57:661-676.

Takeuchi K, Araki H, Umeda M, Komoike Y, and Suzuki K (2001a) Adaptive gastric cytoprotection is mediated by prostaglandin $\mathrm{EP}_{1}$ receptors: a study using rats and knockout mice. J Pharmacol Exp Ther 297:1160-1165.

Takeuchi K, Kato S, Ogawa Y, Kanatsu K, and Umeda M (2001b) Role of endogenous prostacyclin in gastric ulcerogenic and healing responses-a study using IPreceptor knockout mice. J Physiol Paris 95:75-80.

Takeuchi K, Kato S, and Tanaka A (2001c) [Gastrointestinal cytoprotection by prostaglandin E and EP receptor subtypes.] Nippon Yakurigaku Zasshi 117:274282 .

Takeuchi K, Takahashi N, Abe T, Ito O, Tsutsumi E, Taniyama Y, and Abe K (1994) Functional difference between two isoforms of rat kidney prostaglandin receptor $\mathrm{EP}_{3}$ subtype. Biochem Biophys Res Commun 203:1897-1903.

Takeuchi K, Tanaka A, Kato S, Aihara E, and Amagase K (2007) Effect of (S)-4-(115-chloro-2-(4-fluorophenoxy) benzamdio) benzoic acid (CJ-42794), a selective antagonist of prostaglandin $\mathrm{E}$ receptor subtype 4, on ulcerogenic and healing responses in rat gastrointestinal mucosa. J Pharmacol Exp Ther 322:903-912.

Takeuchi K, Ukawa H, Kato S, Furukawa O, Araki H, Sugimoto Y, Ichikawa A Ushikubi F, and Narumiya S (1999) Impaired duodenal bicarbonate secretion and mucosal integrity in mice lacking prostaglandin E-receptor subtype EP3. Gastroenterology 117:1128-1135.

Talpain E, Armstrong RA, Coleman RA, and Vardey CJ (1995) Characterization of the PGE receptor subtype mediating inhibition of superoxide production in human neutrophils. Br J Pharmacol 114:1459-1465.

Tamba S, Yodoi R, Segi-Nishida E, Ichikawa A, Narumiya S, and Sugimoto Y (2008) Timely interaction between prostaglandin and chemokine signaling is a prerequisite for successful fertilization. Proc Natl Acad Sci USA 105:14539-14544

Tan X, Essengue S, Talreja J, Reese J, Stechschulte DJ, and Dileepan KN (2007) Histamine directly and synergistically with lipopolysaccharide stimulates cyclooxygenase- 2 expression and prostaglandin $\mathrm{I}_{2}$ and $\mathrm{E}_{2}$ production in human coronary artery endothelial cells. J Immunol 179:7899-7906.

Tanaka M, Sakai A, Uchida S, Tanaka S, Nagashima M, Katayama T, Yamaguchi K, and Nakamura T (2004) Prostaglandin E2 receptor (EP4) selective agonist (ONO4819.CD) accelerates bone repair of femoral cortex after drill-hole injury associated with local upregulation of bone turnover in mature rats. Bone 34:940-948.

Tanaka T, Fukuta Y, Higashino R, Sato R, Nomura Y, Fukuda Y, Ito S, Takei M, Kurimoto T, and Tamaki H (1998) Antiplatelet effect of Z-335, a new orally active and long-lasting thromboxane receptor antagonist. Eur J Pharmacol 357:53-60.

Tang CH, Yang RS, and Fu WM (2005) Prostaglandin $\mathrm{E}_{2}$ stimulates fibronectin expression through $\mathrm{EP}_{1}$ receptor, phospholipase $\mathrm{C}$, protein kinase $\mathrm{C} \alpha$, and c-Src pathway in primary cultured rat osteoblasts. J Biol Chem 280:22907-22916.

Tani K, Naganawa A, Ishida A, Egashira H, Sagawa K, Harada H, Ogawa M, Maruyama T, Ohuchida S, Nakai H, et al. (2002a) Development of a highly selective $\mathrm{EP}_{2}$-receptor agonist. Part 2: identification of 16-hydroxy-17,17trimethylene 9 $\beta$-chloro-PGF derivatives. Bioorg Med Chem 10:1107-1114.

Tani K, Naganawa A, Ishida A, Sagawa K, Harada H, Ogawa M, Maruyama T, Ohuchida S, Nakai H, Kondo K, et al. (2002b) Development of a highly selective $\mathrm{EP}_{2}$-receptor agonist. Part 1: identification of 16-hydroxy-17,17-trimethylene $\mathrm{PGE}_{2}$ derivatives. Bioorg Med Chem 10:1093-1106.

Taniguchi H, Mohri I, Okabe-Arahori H, Aritake K, Wada K, Kanekiyo T, Narumiya S, Nakayama M, Ozono K, Urade Y, et al. (2007) Prostaglandin D2 protect neonatal mouse brain from hypoxic ischemic injury. $J$ Neurosci 27:4303-4312.

Taniguchi T, Fujino H, Israel DD, Regan JW, and Murayama T (2008) Human EP3 prostanoid receptor induces VEGF and VEGF receptor-1 mRNA expression. Biochem Biophys Res Commun 377:1173-1178.

Tauchi M, Fuchs TA, Kellenberger AJ, Woodward DF, Paus R, and Lütjen-Drecoll E 
(2010) Characterization of an in vivo model for the study of eyelash biology and trichomegaly: mouse eyelash morphology, development, growth cycle, and anagen prolongation by bimatoprost. $\mathrm{Br}$ J Dermatol 162:1186-1197.

Telleria-Diaz A, Ebersberger A, Vasquez E, Schache F, Kahlenbach J, and Schaible HG (2008) Different effects of spinally applied prostaglandin $\mathrm{D}_{2}$ on responses of dorsal horn neurons with knee input in normal rats and in rats with acute knee inflammation. Neuroscience 156:184-192.

Terashima S, Nishio H, Ogura M, Honda M, and Takeuchi K (2009) Involvement of prostacyclin/IP receptors in decreased acid response of damaged stomachsmediation by somatostatin/SST2 receptors. Life Sci 84:172-180.

Thampatty BP, Li H, Im HJ, and Wang JH (2007) $\mathrm{EP}_{4}$ receptor regulates collagen type-1, $M M P-1$, and $M M P-3$ gene expression in human tendon fibroblasts in response to IL-1 $\beta$ treatment. Gene 386:154-161.

Thierauch KH, Stürzebecher CS, Schillinger E, Rehwinkel H, Radüchel B, Skuballa $\mathrm{W}$, and Vorbrüggen $\mathrm{H}$ (1988) Stable $9 \beta$ - or $11 \alpha$-halogen-15-cyclohexylprostaglandins with high affinity to the $\mathrm{PGD}_{2}$-receptor. Prostaglandins 35:855868.

Thomas DW, Mannon RB, Mannon PJ, Latour A, Oliver JA, Hoffman M, Smithies O, Koller BH, and Coffman TM (1998) Coagulation defects and altered hemodynamic responses in mice lacking receptors for thromboxane $\mathrm{A}_{2}$. J Clin Invest 102:19942001.

Thomas DW, Rocha PN, Nataraj C, Robinson LA, Spurney RF, Koller BH, and Coffman TM (2003) Proinflammatory actions of thromboxane receptors to enhance cellular immune responses. J Immunol 171:6389-6395.

Thompson EJ, Gupta A, Vielhauer GA, Regan JW, and Bowden GT (2001) The growth of malignant keratinocytes depends on signaling through the PGE(2) receptor $\mathrm{EP}_{1}$. Neoplasia 3:402-410.

Thura M, Hokamura K, Yamamoto S, Maeda M, Furuta K, Suzuki M, Ibaraki K, and Umemura K (2009) GIF-0173 protects against cerebral infarction through $\mathrm{DP}_{1}$ receptor activation. Exp Neurol 219:481-491.

Tian M and Schiemann WP (2010) PGE 2 receptor EP 2 mediates the antagonistic effect of COX-2 on TGF-beta signaling during mammary tumorigenesis. FASEB J 24:1105-1116.

Tilley SL, Audoly LP, Hicks EH, Kim HS, Flannery PJ, Coffman TM, and Koller BH (1999) Reproductive failure and reduced blood pressure in mice lacking the EP2 prostaglandin E2 receptor. J Clin Invest 103:1539-1545.

Tilley SL, Hartney JM, Erikson CJ, Jania C, Nguyen M, Stock J, McNeisch J, Valancius C, Panettieri RA Jr, Penn RB, et al. (2003) Receptors and pathways mediating the effects of prostaglandin $\mathrm{E}_{2}$ on airway tone. Am J Physiol Lung Cell Mol Physiol 284:L599-L606.

Timoshenko AV, Xu G, Chakrabarti S, Lala PK, and Chakraborty C (2003) Role of prostaglandin $\mathrm{E} 2$ receptors in migration of murine and human breast cancer cells. Exp Cell Res 289:265-274.

Tober KL, Wilgus TA, Kusewitt DF, Thomas-Ahner JM, Maruyama T, and Oberyszyn TM (2006) Importance of the EP(1) receptor in cutaneous UVB-induced inflammation and tumor development. J Invest Dermatol 126:205-211.

Toh H, Ichikawa A, and Narumiya S (1995) Molecular evolution of receptors for eicosanoids. FEBS Lett 361:17-21.

Tommasini I, Cerioni L, Palomba L, and Cantoni O (2008) Prostaglandin $\mathrm{E}_{2}$ signals monocyte/macrophage survival to peroxynitrite via protein kinase A converging in Bad phosphorylation with the protein kinase $\mathrm{C}_{0}$-dependent pathway driven by 5-hydroxyeicosatetraenoic acid. J Immunol 181:5637-5645.

Toris CB, Zhan G, Fan S, Dickerson JE, Landry TA, Bergamini MV, and Camras CB (2007) Effects of travoprost on aqueous humor dynamics in patients with elevated intraocular pressure. J Glaucoma 16:189-195

Toris CB, Zhan GL, Camras CB, and McLaughlin MA (2005) Effects of travoprost on aqueous humor dynamics in monkeys. J Glaucoma 14:70-73.

Toris CB, Zhan GL, Feilmeier MR, Camras CB, and McLaughlin MA (2006) Effects of a prostaglandin DP receptor agonist, AL-6598, on aqueous humor dynamics in a nonhuman primate model of glaucoma. $J$ Ocul Pharmacol Ther 22:86-92.

Torisu K, Hasegawa T, Kobayashi K, and Nambu F (2003a), inventors; Ono Pharm. Co. Ltd., assignee. Indole derivatives, process for producing the same and drugs containing the same as the active ingredient. World patent WO03022813. 2003 March 20.

Torisu K, Iwahashi M, Kobayashi K, and Nambu F (2003b), inventors; Ono Pharm. Co. Ltd., assignee. Indole derivative. World patent WO03022814. 2003 March 20. Torisu K, Kobayashi K, Iwahashi M, Nakai Y, Onoda T, Nagase T, Sugimoto I, Okada Y, Matsumoto R, Nanbu F, et al. (2004) Discovery of a new class of potent, selective, and orally active prostaglandin $\mathrm{D}_{2}$ receptor antagonists. Bioorg Med Chem 12:5361-5378.

Torres D, Paget C, Fontaine J, Mallevaey T, Matsuoka T, Maruyama T, Narumiya S, Capron M, Gosset P, Faveeuw C, et al. (2008) Prostaglandin $\mathrm{D}_{2}$ inhibits the production of INF- $\gamma$ by invariant NK T cells: consequences in the control of B16 melanoma. J Immunol 180:783-792.

Town MH, Casals-Stenzel J, and Schillinger E (1983) Pharmacological and cardiovascular properties of a hydantoin derivative, BW $245 \mathrm{C}$, with high affinity and selectivity for $\mathrm{PGD}_{2}$ receptors. Prostaglandins 25:13-28.

Trist DG, Collins BA, Wood J, Kelly MG, and Robertson AD (1989) The antagonism by $\mathrm{BW} \mathrm{A} 868 \mathrm{C}$ of $\mathrm{PGD}_{2}$ and BW245C activation of human platelet adenylate cyclase. Br J Pharmacol 96:301-306.

Trivedi DB, Sugimoto Y, and Loftin CD (2006) Attenuated cyclooxygenase-2 expression contributes to patent ductus arteriosus in preterm mice. Pediatr Res 60:669674.

Tsuchiya H, Oka T, Nakamura K, Ichikawa A, Saper CB, and Sugimoto Y (2008) Prostaglandin E2 attenuates preoptic expression of GABAA receptors via EP3 receptors. J Biol Chem 283:11064-11071.

Tsujisawa T, Inoue H, and Nishihara T (2005) SC-19220, antagonist of prostaglandin $\mathrm{E}_{2}$ receptor $\mathrm{EP}_{1}$, inhibits osteoclastogenesis by RANKL. J Bone Miner Res 20:15-22.

Tsukamoto M, Kiso T, Shimoshige Y, Aoki T, and Matsuoka N (2010) Spinal mechanisms of standard analgesics: evaluation using mouse models of allodynia. Eur J Pharmacol 634:40-45.
Tsutsumi R, Xie C, Wei X, Zhang M, Zhang X, Flick LM, Schwarz EM, and O'Keefe RJ (2009) $\mathrm{PGE}_{2}$ signaling through the EP4 receptor on fibroblasts upregulates RANKL and stimulates osteolysis. J Bone Miner Res 24:1753-1762.

Tymkewycz PM, Jones RL, Wilson NH, and Marr CG (1991) Heterogeneity of thromboxane $\mathrm{A}_{2}$ (TP-) receptors: evidence from antagonist but not agonist potency measurements. Br J Pharmacol 102:607-614.

Uchiyama K, Saito M, Sasaki M, Obara Y, Higashiyama S, and Nakahata N (2009) Thromboxane $\mathrm{A}_{2}$ receptor-mediated epidermal growth factor receptor transactivation: involvement of PKC- $\delta$ and $\mathrm{PkC}-\varepsilon$ in the shedding of epidermal growth factor receptor ligands. Eur J Pharm Sci 38:504-511.

Ueno A, Matsumoto H, Naraba H, Ikeda Y, Ushikubi F, Matsuoka T, Narumiya S, Sugimoto Y, Ichikawa A, and Oh-ishi S (2001) Major roles of prostanoid receptor IP and $\operatorname{EP}(3)$ in endotoxin-induced enhancement of pain. Biochem Pharmacol 62:157-160.

Ueno A, Naraba H, Ikeda Y, Ushikubi F, Murata T, Narumiya S, and Oh-ishi S (2000) Intrinsic prostacyclin contributes to exudation induced by bradykinin or carrageenin: a study on the paw edema induced in IP-receptor-deficient mice. Life Sci 66:PL155-PL160.

Uller L, Mathiesen JM, Alenmyr L, Korsgren M, Ulven T, Högberg T, Andersson G, Persson CG, and Kostenis E (2007) Antagonism of the prostaglandin D2 receptor CRTH2 attenuates asthma pathology in mouse eosinophilic airway inflammation. Respir Res 28:16.

Ulven T and Kostenis E (2005) Minor structural modifications convert the dual TP/CRTH2 antagonist ramatroban into a highly selective and potent CRTH2 antagonist. J Med Chem 48:897-900.

Ungrin MD, Carrière MC, Denis D, Lamontagne S, Sawyer N, Stocco R, Tremblay N, Metters KM, and Abramovitz M (2001) Key structural features of prostaglandin $\mathrm{E}_{2}$ and prostanoid analogs involved in binding and activation of the human $\mathrm{EP}_{1}$ prostanoid receptor. Mol Pharmacol 59:1446-1456.

Uno H, Zimbric ML, Albert DM, and Stjernschantz J (2002) Effect of latanoprost on hair growth in the bald scalp of the stump-tailed macacque: a pilot study. Acta Derm Venereol 82:7-12.

Urade Y and Hayaishi O (1999) Prostaglandin $\mathrm{D}_{2}$ and sleep regulation. Biochem Biophys Acta 1436:606-615.

Ushikubi F, Aiba Y, Nakamura K, Namba T, Hirata M, Mazda O, Katsura Y, and Narumiya S (1993) Thromboxane A2 receptor is highly expressed in mouse immature thymocytes and mediates DNA fragmentation and apoptosis. $J$ Exp Med 178:1825-1830.

Ushikubi F, Nakajima M, Hirata M, Okuma M, Fujiwara M, and Narumiya S (1989) Purification of the thromboxane $\mathrm{A}_{2} /$ prostaglandin $\mathrm{H}_{2}$ receptor from human platelets. J Biol Chem 264:16496-16501.

Ushikubi F, Nakamura K, and Narumiya S (1994) Functional reconstitution of platelet thromboxane $\mathrm{A} 2$ receptors with $\mathrm{G}_{\mathrm{q}}$ and $\mathrm{G}_{12}$ in phospholipd vesicles. Mol Pharmacol 46:808-816.

Ushikubi F, Segi E, Sugimoto Y, Murata T, Matsuoka T, Kobayashi T, Hizaki H, Tuboi K, Katsuyama M, Ichikawa A, et al. (1998) Impaired febrile responses in mice lacking the prostaglandin $\mathrm{E}$ receptor subtype $\mathrm{EP}_{3}$. Nature 395:281-284.

Väänänen JE, Väänänen CC, Lee S, Yuen BH, and Leung PC (1998) Regulation of prostaglandin $\mathrm{F}_{2 \alpha}$-receptor mRNA in human granulosa-luteal cells by human chorionic gonadotrophin and prostaglandin $\mathrm{F}_{2 \alpha}$. Endocrine 8:261-267.

van Rodijnen WF, Korstjens IJ, Legerstee N, Ter Wee PM, and Tangelder GJ (2007) Direct vasoconstrictor effect of prostaglandin $\mathrm{E}_{2}$ on renal interlobular arteries: role of the $\mathrm{EP}_{3}$ receptor. Am J Physiol Renal Physiol 292:F1094-F1101.

Vassiliou E, Jing H, and Ganea D (2003) Prostaglandin $\mathrm{E}_{2}$ inhibits TNF production in murine bone marrow-derived dendritic cells. Cell Immunol 223:120-132.

Vezza R, Habib A, and FitzGerald GA (1999) Differential signaling by the thromboxane receptor isoforms via the novel GTP-binding protein, Gh. J Biol Chem 274:12774-12779.

Vezza R, Mezzasoma AM, Venditti G, and Gresele P (2002) Prostaglandin endoperoxides and thromboxane $\mathrm{A}_{2}$ activate the same receptor isoforms in human platelets. Thromb Haemost 87:114-121.

Vysniauskiene I, Allemann R, Flammer J, and Haefliger IO (2006) Vasoactive responses to U-46619, $\mathrm{PGF}_{2 \alpha}$, latanoprost, and travoprost in isolated porcine ciliary arteries. Invest Ophthalmol Vis Sci 47:295-298.

Wacker MJ, Kosloski LM, Gilbert WJ, Touchberry CD, Moore DS, Kelly JK, Brotto $\mathrm{M}$, and Orr JA (2009) Inhibition of thromboxane $\mathrm{A}_{2}$-induced arrhythmias and intracellular calcium changes in cardiac myocytes by blockade of the inosito trisphosphate pathway. J Pharmacol Exp Ther 331:917-924.

Walker W and Rotondo D (2004) Prostaglandin $\mathrm{E}_{2}$ is a potent regulation of interleukin-12-and interleukin-18-induced natural killer cell interferon- $\gamma$ synthesis. Immunology 111:298-305.

Walsh MT, Foley JF, and Kinsella BT (1998) Characterization of the role of N-linked glycosylation on the cell signaling and expression of the human thromboxane $\mathrm{A}_{2}$ receptor alpha and beta isoforms. J Pharmacol Exp Ther 286:1026-1036.

Wan Z, Woodward DF, Cornell CL, Fliri HG, Martos JL, Pettit SN, Wang JW, Kharlamb AB, Wheeler LA, Garst ME, et al. (2007) Bimatoprost, prostamide activity, and conventional drainage. Invest Ophthalmol Vis Sci 48:4107-4115.

Wang D, Wong D, Wang M, Cheng Y, and Fitzgerald GA (2005) Cardiovascular hazard and non-steroidal anti-inflammatory drugs. Curr Opin Pharmacol 5:204210 .

Wang XS and Lau HY (2006) Prostaglandin E potentiates the immunologically stimulated histamine release from human peripheral blood-derived mast cells through $\mathrm{EP}_{1} / \mathrm{EP}_{3}$ receptors. Allergy 61:503-506.

Wasserman MA (1981) Pharmacologic and therapeutic implications of prostaglandins on airway smooth muscle. Bull Eur Physiopathol Respir 17:675-686.

Watabe A, Sugimoto Y, Honda A, Irie A, Namba T, Negishi M, Ito S, Narumiya S, and Ichikawa A (1993) Cloning and expression of cDNA for a mouse $\mathrm{EP}_{1}$ subtype of prostaglandin E receptor. J Biol Chem 268:20175-20178.

Watanabe H, Katoh T, Eiro M, Iwamoto M, Ushikubi F, Narumiya S, and Watanabe $\mathrm{T}$ (2005) Effects of salt loading on blood pressure in mice lacking the prostanoid receptor gene. Circ J 69:124-126. 
Watanabe K, Kawamori T, Nakatsugi S, Ohta T, Ohuchida S, Yamamoto H, Maruyama T, Kondo K, Narumiya S, Sugimura T, et al. (2000) Inhibiting effect of a prostaglandin E receptor subtype EP(1) selective antagonist, ONO-8713, on development of azoxymethane-induced aberrant crypt foci in mice. Cancer Lett 156:57-61.

Watanabe K, Kawamori T, Nakatsugi S, Ohta T, Ohuchida S, Yamamoto H, Maruyama T, Kondo K, Ushikubi F, Narumiya S, et al. (1999) Role of the prostaglandin $\mathrm{E}$ receptor subtype $\mathrm{EP}_{1}$ in colon carcinogenesis. Cancer Res 59:5093-5096.

Wei G, Kibler KK, Koehler RC, Maruyama T, Narumiya S, and Doré S (2008) Prostacyclin receptor deletion aggravates hippocampal neuronal loss after bilateral common carotid artery occlusion in mouse. Neuroscience 156:1111-1117.

Wei J, Yan W, Li X, Ding Y, and Tai HH (2010) Thromboxane receptor $\alpha$ mediates tumor growth and angiogenesis via induction of vascular endothelial growth factor expression in human lung cancer cells. Lung Cancer 69:26-32.

Weichman BM, Muccitelli RM, Osborn RR, Holden DA, Gleason JG, and Wasserman MA (1982) In vitro and in vivo mechanisms of leukotriene-mediated bronchoconstriction in the guinea pig. J Pharmacol Exp Ther 222:202-208.

Weinberg E, Zeldich E, Weinreb MM, Moses O, Nemcovsky C, and Weinreb M (2009) Prostaglandin $\mathrm{E}_{2}$ inhibits the proliferation of human gingival fibroblasts via $\mathrm{EP}_{2}$ receptor and Epac. J Cell Biochem 108:207-215.

Weinreb RN and Lindsey JD (2002) Metalloproteinase gene transcription in human ciliary muscle cells with latanoprost. Invest Ophthalmol Vis Sci 43:716-722.

Welburn PJ and Jones RL (1978) A comparison of prostaglndin $\mathrm{F}_{2 \alpha}$ and three 16-aryloxy analogues on isolated rabbit jejunum. Prostaglandins 15:287-296.

Weller CL, Collington SJ, Hartnell A, Conroy DM, Kaise T, Barker JE, Wilson MS, Taylor GW, Jose PJ, and Williams TJ (2007) Chemotactic action of prostaglandin $\mathrm{E}_{2}$ on mouse mast cells acting via the $\mathrm{PGE}_{2}$ receptor 3. Proc Natl Acad Sci USA 104:11712-11717.

Wheeldon A and Vardey CJ (1993) Characterization of the inhibitory prostanoid receptors on human neutrophils. Br J Pharmacol 108:1051-1054.

White ES, Atrasz RG, Dickie EG, Aronoff DM, Stambolic V, Mak TW, Moore BB, and Peters-Golden M (2005) Prostaglandin $\mathrm{E}_{2}$ inhibits fibroblast migration by E-prostanoid 2 receptor-mediated increase in PTEN activity. Am J Respir Cell Mol Biol 32:135-141.

Whittle BJ, Moncada S, Mullane K, and Vane JR (1983) ) Platelet and cardiovascular activity of the hydantoin BW245C, a potent prostaglandin analogue. Prostaglandins 25:205-223.

Whittle BJ, Moncada S, and Vane JR (1978) Comparison of the effects of prostacyclin $\left(\mathrm{PGI}_{2}\right)$, prostaglandin $\mathrm{E}_{1}$ and $\mathrm{D}_{2}$ on platelet aggregation in different species. Prostaglandins 16:373-388.

Wikland M, Lindblom B, and Wiqvist N (1984) Myometrial response to prostaglandins during labor. Gynecol Obstet Invest 17:131-138.

Wilson NH and Jones RL (1985) Prostaglandin endoperoxide and thromboxane A analogues. Adv Prostaglandin Thromboxane Leukot Res 14:393-425.

Wilson NH, Jones RL, Marr CG, and Muir G (1988) Synthesis of oxabicyclo[2.2.1]heptane prostanoids having thromboxane-like activity at subnanomolar concentrations. Eur J Med Chem 23:359-364.

Wilson SJ, McGinley K, Huang AJ, and Smyth EM (2007) Heterodimerization of the $\alpha$ and $\beta$ isoforms of the human thromboxane receptor enhances isoprostane signaling. Biochem Biophys Res Commun 352:397-403.

Wilson SJ, Roche AM, Kostetskaia E, and Smyth EM (2004) Dimerization of the human receptors for prostacyclin and thromboxane facilitates thromboxane receptor-mediated cAMP generation. J Biol Chem 279:53036-53047.

Wise H and Jones RL (1996) Focus on prostacyclin and its novel mimetics. Trends Pharmacol Sci 17:17-21.

Wlodawer P, Samuelsson B, Albonico SM, and Corey EJ (1971) Selective inhibition of prostaglandin synthetase by a bicyclo[2.2.1]heptene derivative. J Am Chem Soc 93:2815-2816

Woodward DF, inventor; Allergan, Inc., assignee (2000) $\mathrm{EP}_{2}$-receptor agonist as neuroprotective agents for the eye. U.S. Patent 6,090,847. 2000 Jul 18 .

Woodward DF, Carling RW, Cornell CL, Fliri HG, Martos JL, Pettit SN, Liang Y, and Wang JW (2008) The pharmacology and therapeutic relevance of endocannabinoid derived cyclo-oxygenase (COX)-2 products. Pharmacol Ther 120:71-80.

Woodward DF and Chen J (2004) Pharmacological exploitation: eicosanoids and their analogues, in The Eicosanoids (Curtis-Prior P ed), pp 613-616, John Wiley \& Sons Ltd.

Woodward DF and Chen J (2007) $\mathrm{EP}_{2}$ receptor agonists for treating glaucoma. U.S. patent 2007/0049625. 2007 Mar 1.

Woodward DF, Fairbairn CE, Krauss AH, Lawrence RA, and Protzman CE (1995a) Radioligand binding analysis of receptor subtypes in two FP receptor preparations that exhibit different functional rank orders of potency in response to prostaglandins. J Pharmacol Exp Ther 273:285-287.

Woodward DF, Hawley SB, Williams LS, Ralston TR, Protzman CE, Spada CS, and Nieves AL (1990) Studies on the ocular pharmacology of prostaglandin $\mathrm{D}_{2}$. Invest Ophthalmol Vis Sci 31:138-146.

Woodward DF, Krauss AH, Chen J, Gil DW, Kedzie KM, Protzman CE, Shi L, Chen R, Krauss HA, Bogardus A, et al. (2000) Replacement of the carboxylic acid group of prostaglandin $\mathrm{F}_{2 \alpha}$ with a hydroxyl or methoxy substituent provides biologically unique compounds. Br J Pharmacol 130:1933-1943.

Woodward DF, Krauss AH, Chen J, Liang Y, Li C, Protzman CE, Bogardus A, Chen R, Kedzie KM, Krauss HA, et al. (2003) Pharmacological characterization of a novel antiglaucoma agent, Bimatoprost (AGN 192024). J Pharmacol Exp Ther 305:772-785.

Woodward DF, Krauss AH, Wang JW, Protzman CE, Nieves AL, Liang Y, Donde Y, Burk RM, Landsverk K, and Struble C (2007) Identification of an antagonist that selectively blocks the activity of prostamides (prostaglandin-ethanolamides) in the feline iris. Br J Pharmacol 150:342-352.

Woodward DF and Lawrence RA (1994) Identification of a single (FP) receptor associated with prostanoid-induced $\mathrm{Ca}^{2+}$ signals in swiss $3 \mathrm{~T} 3$ cells. Biochem Pharmacol 47:1567-1574.

Woodward DF, Nilsson SF, Toris CB, Kharlamb AB, Nieves AL, and Krauss AH (2009) Prostanoid $\mathrm{EP}_{4}$ receptor stimulation produces ocular hypotension by a mechanism that does not appear to involve uveoscleral outflow. Invest Ophthalmo Vis Sci 50:3320-3328.

Woodward DF, Pepperl DJ, Burkey TH, and Regan JW (1995b) 6-Isopropoxy-9 oxoxanthene-2-carboxylic acid (AH 6809), a human EP2 receptor antagonist. Biochem Pharmacol 50:1731-1733.

Woodward DF, Protzman CE, Krauss AH, and Williams LS (1993a) Identification of 19(R)-OH prostaglandin $\mathrm{E}_{2}$ as a selective prostanoid $\mathrm{EP}_{2}$-receptor agonist. Pros taglandins 46:371-383.

Woodward DF, Spada CS, Hawley SB, Williams LS, Protzman CE, and Nieves AL (1993b) Further studies on ocular responses to DP receptor stimulation. Eur J Pharmacol 230:327-333.

Wright DH, Metters KM, Abramovitz M, and Ford-Hutchinson AW (1998) Characterization of the recombinant human prostanoid DP receptor and identification of L-644,698, a novel selective DP agonist. Br J Pharmacol 123:1317-1324.

Wright WS, McElhatten RM, and Harris NR (2009a) Expression of thromboxane synthase and the thromboxane-prostanoid receptor in mouse and rat retina. Exp Eye Res 89:532-537.

Wright WS, Messina JE, and Harris NR (2009b) Attenuated of diabetes-induced retinal vasoconstriction by a thromboxane receptor antagonist. Exp Eye Res $\mathbf{8 8}$ $106-112$

Wu L, Wang Q, Liang X, and Andreasson K (2007) Divergent effects of prostaglandin receptor signaling on neuronal survival. Neurosci Lett 421:253-258.

Xiang A, Uchida Y, Nomura A, Iijima H, Sakamoto T, Ishii Y, Morishima Y, Masuyama K, Zhang M, Hirano K, et al. (2002) Involvement of thromboxane $\mathrm{A}_{2}$ in airway mucous cells in asthma-related cough. J Appl Physiol 92:763-770.

Xiao CY, Hara A, Yuhki K, Fujino T, Ma H, Okada Y, Takahata O, Yamada T, Murata T, Narumiya S, et al. (2001) Roles of prostaglandin $I_{2}$ and thromboxane $A_{2}$ in cardiac ischemia-reperfusion injury: a study using mice lacking their respective receptors. Circulation 104:2210-2215.

Xiao CY, Yuhki K, Hara A, Fujino T, Kuriyama S, Yamada T, Takayama K, Takahata O, Karibe H, Taniguchi T, et al. (2004) Prostaglandin $\mathrm{E}_{2}$ protects the hear from ischemia-reperfusion injury via its receptor subtype $\mathrm{EP}_{4}$. Circulation 109 $2462-2468$.

Xu W, Chou CL, Israel DD, Hutchinson AJ, and Regan JW (2009) PGF P $_{2 \alpha}$ stimulate FP prostanoid receptor mediated crosstalk between Ras/Raf signaling and Tcf transcriptional activation. Biochem Biophys Res Commun 381:625-629.

Xu W, Chou CL, Sun H, Fujino H, Chen QM, and Regan JW (2008) FP prostanoid receptor-mediated induction of the expression of early growth response factor- 1 by activation of a Ras/Raf/mitogen-activated protein kinase signaling cascade. $\mathrm{Mol}$ Pharmacol 73:111-118

Xue L, Gyles SL, Barrow A, and Pettipher R (2007) Inhibition of PI3K and calcineurin suppresses chemoattractant receptor-homologous molecule expressed on Th cells (CRTH2)-dependent responses of Th2 lymphocytes to prostaglandin $\mathrm{D}_{2}$ Biochem Pharmacol 73:843-853.

Xue L, Gyles SL, Wettey FR, Gazi L, Townsend E, Hunter MG, and Pettipher R (2005) Prostaglandin $\mathrm{D}_{2}$ causes preferential induction of proinflammatory Th2 cytokine production through an action on chemoattractant receptor-like molecule expressed on Th2 cells. J Immunol 175:6531-6536.

Yamaguchi DT, Hahn TJ, Beeker TG, Kleeman CR, and Muallem S (1988) Relationship of cAMP and calcium messenger systems in prostaglandin-stimulated UMR 106 cells. $J$ Biol Chem $\mathbf{2 6 3 : 1 0 7 4 5 - 1 0 7 5 3 . ~}$

Yamaguchi T, Kubota T, Watanabe S, and Yamamoto T (2004) Activation of brain prostanoid $\mathrm{EP}_{3}$ receptors via arachidonic acid cascade during behavioral suppression induced by Delta8-tetrahydrocannabinol. J Neurochem 88:148-154.

Yamaki F, Kaga M, Horinouchi T, Tanaka H, Koike K, Shigenobu K, Toro L, and Tanaka Y (2001) MaxiK channel-mediated relaxation of guinea-pig aorta following stimulation of IP receptor with beraprost via cyclic AMP-dependent and -independent mechanism. Naunyn Schmiedebergs Arch Pharmacol 364:538-550.

Yamane H, Sugimoto Y, Tanaka S, and Ichikawa A (2000) Prostaglandin E receptors, $\mathrm{EP}_{2}$ and $\mathrm{EP}_{4}$, differentially modulate TNF- $\alpha$ and IL-6 production induced by lipopolysaccharide in mouse peritoneal neutrophils. Biochem Biophys Res Com mun 278:224-228

Yamaoka K, Yano A, Kuroiwa K, Morimoto K, Inazumi T, Hatae N, Tabata $\mathrm{H}$ Segi-Nishida E, Tanaka S, Ichikawa A, et al. (2009) Prostaglandin $\mathrm{EP}_{3}$ receptor superactivates adenylyl cyclase via $\mathrm{G}_{\mathrm{q}} / \mathrm{PLC} / \mathrm{Ca}^{2+}$ pathway in a lipid raftdependent manner. Biochem Biophys Res Commun 389:678-682.

Yamato M, Nagahama K, Kotani T, Kato S, and Takeuchi K (2005) Biphasic effect of prostaglandin $\mathrm{E}_{2}$ in a rat model of esophagitis mediated by $\mathrm{EP}_{1}$ receptors: relation to pepsin secretion. Digestion 72:109-118.

Yanagisawa A, Smith JA, Brezinski ME, and Lefer AM (1987) Mechanism of antagonism of thromboxane receptors in vascular smooth muscle. Eur J Pharmaco 133:89-96

Yang H, Zhang J, Breyer RM, and Chen C (2009) Altered hippocampal long-term synaptic plasticity in mice deficient in the PGE2 EP2 receptor. J Neurochem 108:295-304.

Yang L, Huang Y, Porta R, Yanagisawa K, Gonzalez A, Segi E, Johnson DH, Narumiya S, and Carbone DP (2006) Host and direct antitumor effects and profound reduction in tumor metastasis with selective $\mathrm{EP}_{4}$ receptor antagonism. Cancer Res 66:9665-9672.

Yang L, Yamagata N, Yadav R, Brandon S, Courtney RL, Morrow JD, Shyr Y, Boothby M, Joyce S, Carbone DP, et al. (2003) Cancer associated immunodeficiency and dendritic cell abnormalities mediated by the prostaglandin $\mathrm{EP}_{2}$ receptor. $J$ Clin Invest 111:727-735.

Yanni SE, Barnett JM, Clark ML, and Penn JS (2009) The role of $\mathrm{PGE}_{2}$ receptor $\mathrm{EP}_{4}$ in pathologic ocular angiogenesis. Invest Ophthalmol Vis Sci 50:5479-5486.

Yao C, Sakata D, Esaki Y, Li Y, Matsuoka T, Kuroiwa K, Sugimoto Y, and Narumiya $\mathrm{S}$ (2009) Prostaglandin $\mathrm{E}_{2}-\mathrm{EP}_{4}$ signaling promotes immune inflammation through Th1 cell differentiation and Th17 cell expansion. Nature Med 15:633-640.

Yokotani K, Okuma Y, and Osumi Y (1996) Inhibition of vagally mediated gastric acid secretion by activation of central prostanoid $\mathrm{EP}_{3}$ receptors in urethaneanaesthetized rats. Br J Pharmacol 117:653-656. 
Yoshida K, Nakamura K, Matsumura K, Kanosue K, König M, Thiel HJ, Boldogköi Z, Toth I, Roth J, Gerstberger R, et al. (2003) Neurons of the rat preoptic area and the raphe pallidus nucleus innervating the brown adipose tissue express the prostaglandin E receptor subtype $\mathrm{EP}_{3}$. Eur J Neurosci 18:1848-1860.

Yoshida K, Oida H, Kobayashi T, Maruyama T, Tanaka M, Katayama T, Yamaguchi K, Segi E, Tsuboyama T, Matsushita M, et al. (2002) Stimulation of bone formation and prevention of bone loss by prostaglandin $\mathrm{E} \mathrm{EP}_{4}$ receptor activation. Proc Nat Acad Sci USA 99:4580-4585.

Yoshida T, Sakamoto H, Horiuchi T, Yamamoto S, Suematsu A, Oda H, and Koshihara $\mathrm{Y}(2001)$ Involvement of prostaglandin $\mathrm{E}_{2}$ in interleukin- $1 \alpha$-induced parathyroid hormone-related peptide production in synovial fibroblasts of patients with rheumatoid arthritis. J Clin Endocrinol Metab 86:3272-3278.

Yoshimura-Uchiyama C, Iikura M, Yamaguchi M, Nagase H, Ishii A, Matsushima K, Yamamoto K, Shichijo M, Bacon KB, and Hirai K (2004) Differential modulation of human basophil functions through prostaglandin $\mathrm{D}_{2}$ receptors $\mathrm{DP}$ and chemoattractant receptor-homologous molecule expressed on Th2 cells/ $/ \mathrm{DP}_{2}$. Clin Exp Allergy 34:1283-1290.

Young RN, Billot X, Han Y, Slipetz DA, Chauret N, Belley M, Metters K, Mathieu MC, Greig GM, Denis D, et al. (2004) Discovery and synthesis of a potent, selective and orally bioavailable EP4 receptor agonist. Heterocycles 64:437-446.

Yu L, Wu WK, Li ZJ, Li HT, Wu YC, and Cho CH (2009a) Prostaglandin E(2) promotes cell proliferation via protein kinase C/extracellular signal regulated kinase pathway-dependent induction of c-Myc expression in human esophageal squamous cell carcinoma cells. Int $J$ Cancer 125:2540-2546.

Yu L, Wu WK, Li ZJ, Wong HP, Tai EK, Li HT, Wu YC, and Cho CH (2008) E series of prostaglandin receptor 2-mediated activation of extracellular signal-regulated kinase/activator protein-1 signaling is required for the mitogenic action of prostaglandin $\mathrm{E}_{2}$ in esophageal squamous-cell carcinoma. J Pharmacol Exp Ther 327: $258-267$

Yu Y, Lucitt MB, Stubbe J, Cheng Y, Friis UG, Hansen PB, Jensen BL, Smyth EM, and FitzGerald GA (2009b) Prostaglandin $\mathrm{F}_{2 \alpha}$ elevates blood pressure and promotes atherosclerosis. Proc Natl Acad Sci USA 106:7985-7990.

Yuan A, Steigen SE, Goll R, Vonen B, Husbekk A, Cui G, and Florholmen J (2008 Dendritic cell infiltration pattern along the colorectal adenoma-carcinoma sequence. APMIS 116:445-456

Yuhki K, Ueno A, Naraba H, Kojima F, Ushikubi F, Narumiya S, and Oh-ishi S (2004) Prostaglandin receptors $\mathrm{EP}_{2}, \mathrm{EP}_{3}$, and IP mediate exudate formation in carrageenin-induced mouse pleurisy. J Pharmacol Exp Ther 311:1218-1224.

Yuhki K, Ushikubi F, Naraba H, Ueno A, Kato H, Kojima F, Narumiya S, Sugimoto Y, Matsushita M, and Oh-Ishi S (2008) Prostaglandin $\mathrm{I}_{2}$ plays a key role in zymosan-induced mouse pleurisy. J Pharmacol Exp Ther 325:601-609.

Zaccolo M (2009) cAMP signal transduction in the heart: understanding spatial control for the development of novel therapeutic strategies. $\mathrm{Br} J$ Pharmacol 158:50-60

Zacharowski K, Olbrich A, and Thiemermann C (1999) Reduction of myocardial injury by the $\mathrm{EP}_{3}$ receptor agonist TEI-3356. Role of protein kinase $\mathrm{C}$ and of $\mathrm{K}_{\mathrm{ATP}}$-channels. Eur J Pharmacol 367:33-39.

Zardi EM, Zardi DM, Cacciapaglia F, Dobrina A, Amoroso A, Picardi A, and Afeltra A (2005) Endothelial dysfunction and activation as an expression of disease: role of prostacyclin analogs. Int Immunopharmacol 5:437-459.
Zehender H, Witte EC, Wolff HP, and Patscheke H (1988) Irreversible inhibition of the $\mathrm{TXA}_{2} / \mathrm{PGH}_{2}$ receptor of human platelets by a photoaffinity ligand. Biochem Pharmacol 37:491-495.

Zeng L, An S, and Goetzl EJ (1996) Regulation of expression of matrix metalloproteinase- 9 in early human T cells of the HSB. 2 cultured cell line by the $\mathrm{EP}_{3}$ subtype prostaglandin $\mathrm{E}_{2}$ receptor. $J$ Biol Chem 271:27744-27750.

Zhang J and Rivest S (1999) Distribution, regulation and colocalization of the genes encoding the $\mathrm{EP}_{2^{-}}$and $\mathrm{EP}_{4}-\mathrm{PGE}_{2}$ receptors in the rat brain and neuronal responses to systemic inflammation. Eur $J$ Neurosci 11:2651-2668.

Zhang L, Bastepe M, Jüppner H, and Ruan KH (2006a) Characterization of the molecular mechanisms of the coupling between intracellular loops of prostacyclin receptor with the $\mathrm{C}$-terminal domain of the $\mathrm{G} \alpha$ s protein in human coronary artery smooth muscle cells. Arch Biochem Biophys 454:80-88.

Zhang L, Brass LF, and Manning DR (2009) The $G_{\alpha}$ and $G_{12}$ families of heterotrimeric G proteins report functional selectivity. Mol Pharmacol 75:235-241.

Zhang L, DiLizio C, Kim D, Smyth EM, and Manning DR (2006) The $\mathrm{G}_{12}$ family of G proteins as a reporter of thromboxane $\mathrm{A}_{2}$ receptor activity. Mol Pharmacol 69: $1433-1440$

Zhang L, Wu J, and Ruan KH (2006b) Solution structure of the first intracellular loop of prostacyclin receptor and implication of its interactions with the C-terminal segment of $\mathrm{G} \alpha$ s protein. Biochemistry 45:1734-1744.

Zhang Z, Austin SC, and Smyth EM (2001) Glycosylation of the human prostacyclin receptor: role in ligand binding and signal transduction. Mol Pharmacol 60:480 487.

Zhao M, Li Z, Peng L, Tang YR, Wang C, Zhang Z, and Peng S (2008) A new class of analgesic agents toward prostacyclin receptor inhibition: synthesis, biological studies and QSAR analysis of 1-hydroxyl-2-substituted phenyl-4,4,5,5, tetramethylimidazolines. Eur J Med Chem 43:1048-1058.

Zhao X, Pearson KE, Stephan DA, and Russell P (2003) Effects of prostaglandin analogues on human ciliary muscle and trabecular meshwork cells. Invest Ophthalmol Vis Sci 44:1945-1952.

Zheng Y, Ritzenthaler JD, Sun X, Roman J, and Han S (2009) Prostaglandin E2 stimulates human lung carcinoma cell growth through induction of integrin-linked kinase: the involvement of EP4 and Sp1. Cancer Res 69:896-904.

Zhou P, Qian L, Chou T, and Iadecola C (2008) Neuroprotection by $\mathrm{PGE}_{2}$ receptor $\mathrm{EP}_{1}$ inhibition involves the PTEN/AKT pathway. Neurobiol Dis 29:543-551.

Zhou W, Blackwell TS, Goleniewska K, O'Neal JF, Fitzgerald GA, Lucitt M, Breyer RM, and Peebles RS Jr (2007) Prostaglandin $\mathrm{I}_{2}$ analogs inhibit Th1 and Th2 effector cytokine production by $\mathrm{CD}_{4} \mathrm{~T}$ cells. J Leukoc Biol 81:809-817.

Zhuge X, Arai H, Xu Y, Murayama T, Kobayashi T, Narumiya S, Kita T, and Yokode $\mathrm{M}$ (2006) Protection of atherogenesis in thromboxane $\mathrm{A}_{2}$ receptor-deficient mice is not associated with thromboxane $\mathrm{A}_{2}$ receptor in bone marrow-derived cells. Biochem Biophys Res Commun 351:865-871.

Ziai N, Dolan JW, Kacere RD, and Brubaker RF (1993) The effects on aqueous humor dynamics of PhXA41, a new prostaglandin $\mathrm{F}_{2 \alpha}$ analogue, after topical application in normal and ocular hypertensive human eyes. Arch Ophthalmol 111:1351-1358.

Ziemann C, Riecke A, Rüdell G, Oetjen E, Steinfelder HJ, Lass C, Kahl GF, and Hirsch-Ernst KI (2006) The role of prostaglandin E receptor-dependent signaling via cAMP in Mdr1b gene activation in primary rat hepatocyte cultures. J Pharmacol Exp Ther 317:378-386. 